\title{
Parent-only interventions for childhood overweight or obesity in children aged 5 to II years (Review)
}

\author{
Loveman E, Al-Khudairy L, Johnson RE, Robertson W, Colquitt JL, Mead EL, Ells LJ, \\ Metzendorf MI, Rees K
}

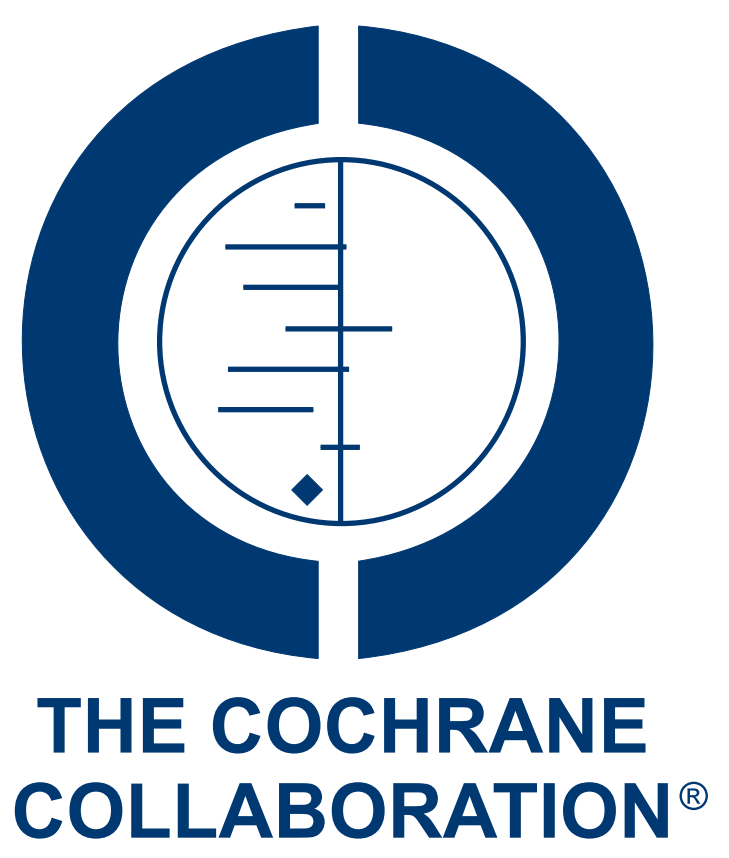

This is a reprint of a Cochrane review, prepared and maintained by The Cochrane Collaboration and published in The Cochrane Library 2015, Issue 12

http://www.thecochranelibrary.com

\section{WILEY}

Parent-only interventions for childhood overweight or obesity in children aged 5 to II years (Review)

Copyright $\odot 2015$ The Cochrane Collaboration. Published by John Wiley \& Sons, Ltd. 
TABLE OF CONTENTS

HEADER . . . . . . . . . . . . . . . . . . . . . . . . . . . . . . . . . . . . . . .

ABSTRACT . . . . . . . . . . . . . . . . . . . . . . . . . . . . . . . . . . . . . . 1

PLAIN LANGUAGE SUMMARY . . . . . . . . . . . . . . . . . . . . . . . . . . . . . . . . . . . . . . . 2

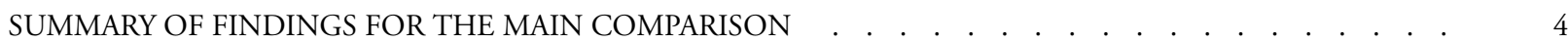

BACKGROUND . . . . . . . . . . . . . . . . . . . . . . . . . . . . . . . . . . . . . . . . . 6

OBJECTIVES . . . . . . . . . . . . . . . . . . . . . . . . . . . . . . . . . . . . . . . . . . . . . . .

METHODS . . . . . . . . . . . . . . . . . . . . . . . . . . . . . . . . . . . . . . . . .

RESULTS . . . . . . . . . . . . . . . . . . . . . . . . . . . . . . . . . . . . . . . 11

Figure 1. . . . . . . . . . . . . . . . . . . . . . . . . . . . . . . . . . . . . . 12

Figure 2. . . . . . . . . . . . . . . . . . . . . . . . . . . . . . . . . . . . . . . 16

Figure 3. . . . . . . . . . . . . . . . . . . . . . . . . . . . . . . . . . . . . . . . . .

ADDITIONAL SUMMARY OF FINDINGS . . . . . . . . . . . . . . . . . . . . . . . . . . . . . . . . . .

DISCUSSION . . . . . . . . . . . . . . . . . . . . . . . . . . . . . . . . . . . . . . . . . . .

AUTHORS' CONCLUSIONS . . . . . . . . . . . . . . . . . . . . . . . . . . . . . . . . . . . . . .

ACKNOWLEDGEMENTS . . . . . . . . . . . . . . . . . . . . . . . . . . . . . . . . . . . . . . . .

REFERENCES . . . . . . . . . . . . . . . . . . . . . . . . . . . . . . . . . . . . . . 34

CHARACTERISTICS OF STUDIES . . . . . . . . . . . . . . . . . . . . . . . . . . . . . . . . 41

DATA AND ANALYSES . . . . . . . . . . . . . . . . . . . . . . . . . . . . . . . . . . . . . . . . 103

Analysis 1.1. Comparison 1 Parent-only interventions versus parent-child interventions, Outcome $1 \mathrm{BMI}$ z score change post intervention. . . . . . . . . . . . . . . . . . . . . . . . . . . . . . . . .

Analysis 1.2. Comparison 1 Parent-only interventions versus parent-child interventions, Outcome 2 BMI z score change longest follow-up. . . . . . . . . . . . . . . . . . . . . . . . . . . . . . . . . . . . . . . . .

Analysis 2.1. Comparison 2 Parent-only interventions versus waiting list interventions, Outcome $1 \mathrm{BMI} z$ score change post intervention.

Analysis 2.2. Comparison 2 Parent-only interventions versus waiting list interventions, Outcome 2 BMI z score change longest follow-up. . . . . . . . . . . . . . . . . . . . . . . . . . . . . . . . . . . . . . . . . . .

Analysis 2.3. Comparison 2 Parent-only interventions versus waiting list interventions, Outcome 3 BMI percentile change post intervention.

Analysis 2.4. Comparison 2 Parent-only interventions versus waiting list interventions, Outcome 4 BMI percentile change longest follow-up.

Analysis 2.5. Comparison 2 Parent-only interventions versus waiting list interventions, Outcome 5 BMI change post intervention.

Analysis 2.6. Comparison 2 Parent-only interventions versus waiting list interventions, Outcome 6 BMI change longest follow-up.

Analysis 3.1. Comparison 3 Parent-only interventions versus minimal contact interventions, Outcome 1 BMI z score change post intervention.

Analysis 3.2. Comparison 3 Parent-only interventions versus minimal contact interventions, Outcome 2 BMI z score change longest follow-up. . . . . . . . . . . . . . . . . . . . . . . . . . . . . . . . . . . . . .

Analysis 3.3. Comparison 3 Parent-only interventions versus minimal contact interventions, Outcome 3 BMI percentile change post intervention. . . . . . . . . . . . . . . . . . . . . . . . . . . . . . . . . . .

Analysis 3.4. Comparison 3 Parent-only interventions versus minimal contact interventions, Outcome 4 BMI percentile change longest follow-up. . . . . . . . . . . . . . . . . . . . . . . . . . . . . . . . . . .

Analysis 3.5. Comparison 3 Parent-only interventions versus minimal contact interventions, Outcome 5 BMI change post intervention.

Analysis 3.6. Comparison 3 Parent-only interventions versus minimal contact interventions, Outcome 6 BMI change longest follow-up.

Analysis 4.1. Comparison 4 Parent-only intervention versus parent-only intervention, Outcome $1 \mathrm{BMI}$ z score change post intervention.

Analysis 4.2. Comparison 4 Parent-only intervention versus parent-only intervention, Outcome 2 BMI z score change longest follow-up. 
Analysis 4.3. Comparison 4 Parent-only intervention versus parent-only intervention, Outcome 3 BMI change post intervention. . . . . . . . . . . . . . . . . . . . . . . . . . . . . . . . . . .

Analysis 4.4. Comparison 4 Parent-only intervention versus parent-only intervention, Outcome 4 BMI change longest follow-up.

Analysis 4.5. Comparison 4 Parent-only intervention versus parent-only intervention, Outcome 5 BMI percentile change post intervention [\%].

ADDITIONAL TABLES . . . . . . . . . . . . . . . . . . . . . . . . . . . . . . . . . . . . . . . . . . . . 121

APPENDICES . . . . . . . . . . . . . . . . . . . . . . . . . . . . . . . . . . . . . . . . .

HISTORY . . . . . . . . . . . . . . . . . . . . . . . . . . . . . . . . . . . . . . . . .

CONTRIBUTIONS OF AUTHORS . . . . . . . . . . . . . . . . . . . . . . . . . . . . . . . . . . . . . . . . . . .

DECLARATIONS OF INTEREST . . . . . . . . . . . . . . . . . . . . . . . . . . . . . . . . . . . . . . . .

SOURCES OF SUPPORT . . . . . . . . . . . . . . . . . . . . . . . . . . . . . . . . . . . . . . . . . . . . .

DIFFERENCES BETWEEN PROTOCOL AND REVIEW . . . . . . . . . . . . . . . . . . . . . . . . . 205

NOTES . . . . . . . . . . . . . . . . . . . . . . . . . . . . . . . . . . . . . . . . . . . . 


\title{
[Intervention Review]
}

\section{Parent-only interventions for childhood overweight or obesity in children aged 5 to II years}

\author{
Emma Loveman ${ }^{1}$, Lena Al-Khudairy ${ }^{2}$, Rebecca E Johnson ${ }^{2}$, Wendy Robertson ${ }^{2}$, Jill L Colquitt ${ }^{1}$, Emma L Mead ${ }^{3}$, Louisa J Ells ${ }^{3}$, \\ Maria-Inti Metzendorf ${ }^{4}$, Karen Rees ${ }^{2}$ \\ ${ }^{1}$ Effective Evidence LLP, Eastleigh, UK. ${ }^{2}$ Division of Health Sciences, Warwick Medical School, University of Warwick, Coventry, UK. \\ ${ }^{3}$ Health and Social Care Institute, Teesside University, Middlesbrough, UK. ${ }^{4}$ Cochrane Metabolic and Endocrine Disorders Group, \\ Institute of General Practice, Medical Faculty of the Heinrich-Heine-University Düsseldorf, Düsseldorf, Germany \\ Contact address: Karen Rees, Division of Health Sciences, Warwick Medical School, University of Warwick, Coventry, CV4 7AL, UK. \\ Karen.Rees@warwick.ac.uk. rees_karen@yahoo.co.uk. \\ Editorial group: Cochrane Metabolic and Endocrine Disorders Group. \\ Publication status and date: New, published in Issue 12, 2015. \\ Review content assessed as up-to-date: 10 March 2015.
}

Citation: Loveman E, Al-Khudairy L, Johnson RE, Robertson W, Colquitt JL, Mead EL, Ells LJ, Metzendorf MI, Rees K. Parentonly interventions for childhood overweight or obesity in children aged 5 to 11 years. Cochrane Database of Systematic Reviews 2015, Issue 12. Art. No.: CD012008. DOI: 10.1002/14651858.CD012008.

Copyright (C) 2015 The Cochrane Collaboration. Published by John Wiley \& Sons, Ltd.

\begin{abstract}
A B S T R A C T
Background

Child and adolescent overweight and obesity have increased globally, and are associated with short- and long-term health consequences.

Objectives

To assess the efficacy of diet, physical activity and behavioural interventions delivered to parents only for the treatment of overweight and obesity in children aged 5 to 11 years.

\section{Search methods}

We performed a systematic literature search of databases including the Cochrane Library, MEDLINE, EMBASE, PsycINFO, CINAHL and LILACS as well trial registers. We checked references of identified trials and systematic reviews. We applied no language restrictions. The date of the last search was March 2015 for all databases.
\end{abstract}

\section{Selection criteria}

We selected randomised controlled trials (RCTs) of diet, physical activity and behavioural interventions delivered to parents only for treating overweight or obesity in children aged 5 to 11 years.

\section{Data collection and analysis}

Two review authors independently assessed trials for risk of bias and evaluated overall study quality using the GRADE instrument. Where necessary, we contacted authors for additional information.

\section{Main results}

We included 20 RCTs, including 3057 participants. The number of participants ranged per trial between 15 and 645 . Follow-up ranged between 24 weeks and two years. Eighteen trials were parallel RCTs and two were cluster RCTs. Twelve RCTs had two comparisons and eight RCTs had three comparisons. The interventions varied widely; the duration, content, delivery and follow-up of the interventions

Parent-only interventions for childhood overweight or obesity in children aged 5 to II years (Review)

Copyright @ 2015 The Cochrane Collaboration. Published by John Wiley \& Sons, Ltd. 
were heterogeneous. The comparators also differed. This review categorised the comparisons into four groups: parent-only versus parent-child, parent-only versus waiting list controls, parent-only versus minimal contact interventions and parent-only versus other parent-only interventions.

Trial quality was generally low with a large proportion of trials rated as high risk of bias on individual risk of bias criteria.

In trials comparing a parent-only intervention with a parent-child intervention, the body mass index (BMI) z score change showed a mean difference (MD) at the longest follow-up period (10 to 24 months) of -0.04 (95\% confidence interval (CI) -0.15 to 0.08 ); $\mathrm{P}$ = 0.56; 267 participants; 3 trials; low quality evidence. In trials comparing a parent-only intervention with a waiting list control, the BMI $\mathrm{z}$ score change in favour of the parent-only intervention at the longest follow-up period (10-12 months) had an MD of - 0.10 (95\% CI -0.19 to -0.01); $\mathrm{P}=0.04 ; 136$ participants; 2 trials; low quality evidence. BMI z score change of parent-only interventions when compared with minimal contact control interventions at the longest follow-up period ( 9 to 12 months) showed an MD of 0.01 (95\% CI -0.07 to 0.09 ); $\mathrm{P}=0.81 ; 165$ participants; 1 trial; low quality evidence. There were few similarities between interventions and comparators across the included trials in the parent-only intervention versus other parent-only interventions and we did not pool these data. Generally, these trials did not show substantial differences between their respective parent-only groups on BMI outcomes.

Other outcomes such as behavioural measures, parent-child relationships and health-related quality of life were reported inconsistently. Adverse effects of the interventions were generally not reported, two trials stated that there were no serious adverse effects. No trials reported on all-cause mortality, morbidity or socioeconomic effects.

All results need to be interpreted cautiously because of their low quality, the heterogeneous interventions and comparators, and the high rates of non-completion.

\section{Authors' conclusions}

Parent-only interventions may be an effective treatment option for overweight or obese children aged 5 to 11 years when compared with waiting list controls. Parent-only interventions had similar effects compared with parent-child interventions and compared with those with minimal contact controls. However, the evidence is at present limited; some of the trials had a high risk of bias with loss to follow-up being a particular issue and there was a lack of evidence for several important outcomes. The systematic review has identified 10 ongoing trials that have a parent-only arm, which will contribute to future updates. These trials will improve the robustness of the analyses by type of comparator, and may permit subgroup analysis by intervention component and the setting. Trial reports should provide adequate details about the interventions to be replicated by others. There is a need to conduct and report cost-effectiveness analyses in future trials in order to establish whether parent-only interventions are more cost-effective than parent-child interventions.

\section{PLAIN LANGUAGE SUMMARY}

\section{Parent-only interventions for childhood overweight or obesity in children aged 5 to 11 years}

\section{Review question}

How effective are diet, physical activity and behavioural interventions delivered to parents only in reducing the weight of overweight and obese children?

\section{Background}

Across the world more children are becoming overweight and obese. These children are more likely to suffer from health problems as children and in later life. Parents can play an important role in determining what their children eat. More information is needed about whether helping parents to make changes to their family's diet and lifestyle will treat this problem.

\section{Study characteristics}

We found 20 randomised controlled trials (clinical studies where people are randomly put into one of two or more treatment groups) comparing diet, physical activity and behavioural (where habits are changed or improved) treatments (interventions) to a variety of control groups (who did not receive treatment) delivered to parents only of 3057 children aged 5 to 11 years. There were few similarities between the trials in the nature and types of interventions used. We grouped the trials by the type of comparisons. Our systematic review reported on the effects of the parent-only interventions compared with parent and child interventions, waiting list controls (where the intervention was delayed until the end of the trial), other interventions with only minimal information or contact and other

Parent-only interventions for childhood overweight or obesity in children aged 5 to I I years (Review) 
types of parent-only interventions. The children in the included trials were monitored (called follow-up) for between six months and two years. This evidence is up to date as of March 2015.

\section{Key results}

The most reported outcome was the body mass index (BMI). This is a measure of body fat and is calculated by dividing weight (in kilograms) by the square of the body height measured in metres $\left(\mathrm{kg} / \mathrm{m}^{2}\right)$. The studies measured BMI in ways that took account of gender, weight and height as the children grew older (such as the BMI z score and the BMI percentile).

When compared with a waiting list control, there was limited evidence that parental interventions helped to reduce BMI. In looking at the longest follow-up periods of the included trials, we did not find firm evidence of an advantage or disadvantage of parent-only interventions when compared with either parent and child interventions, or when compared with limited information. Our review found very little information about how different types of parental interventions compared. No trial reported on death from any cause, illness or socioeconomic effects (such as whether parent-only interventions are lower in costs compared with parent and child interventions). Two trials reported no serious side effects and the rest of the trials did not report whether side effects occurred or not. Information on parent-child relationships and health-related quality of life was rarely reported.

\section{Quality of the evidence}

The overall quality of the evidence was low, mainly because there were just a few trials per measurement or the number of the included children was small. In addition, many children left the trials before they had finished.

Parent-only interventions for childhood overweight or obesity in children aged 5 to I I years (Review) 


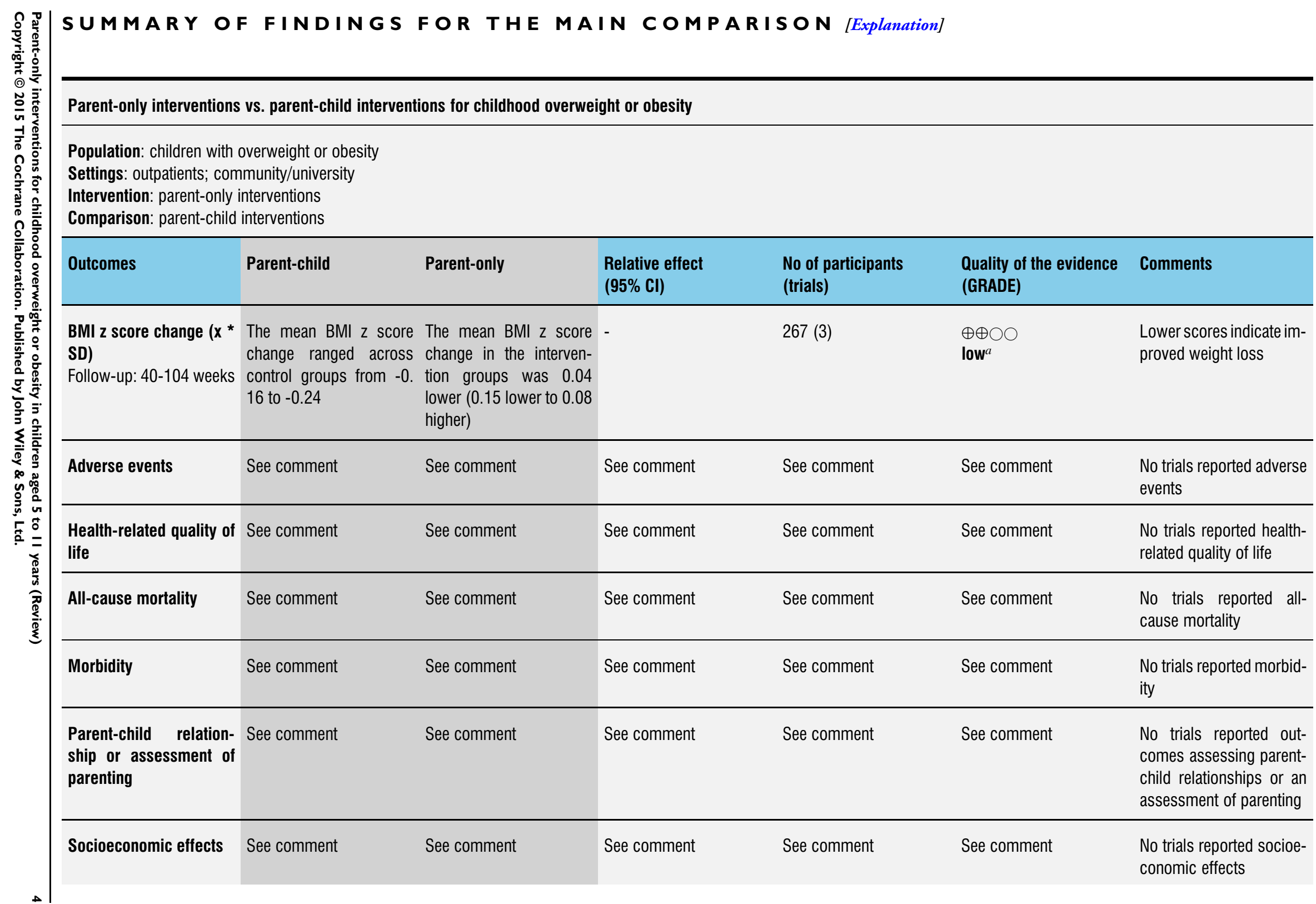


*The basis for the assumed risk (e.g. the median control group risk across trials) is provided in footnotes. The corresponding risk (and its $95 \%$ confidence interval) is based on the assumed risk in the comparison group and the relative effect of the intervention (and its $95 \% \mathrm{Cl}$ ).

BMI: body mass index; Cl: confidence interval; SD: standard deviation.

GRADE Working Group grades of evidence

High quality: Further research is very unlikely to change our confidence in the estimate of effect.

Moderate quality: Further research is likely to have an important impact on our confidence in the estimate of effect and may change the estimate.

Low quality: Further research is very likely to have an important impact on our confidence in the estimate of effect and is likely to change the estimate.

Very low quality: We are very uncertain about the estimate.

' A BMI z score or standard deviation score indicates how many units (of the standard deviation) a child's BMI is above or below the average BMI value for their age group and sex. For instance, a $z$ score of 1.5 indicates that a child' is 1.5 standard deviations above the average value, and a $z$ score of -1.5 indicates a child is 1.5 standard deviations below the average value" (Noo NHS 2011).

${ }^{a}$ Downgraded by one level because of serious risk of attrition bias and one level for serious imprecision (see Appendix 9). 


\section{B A C K G R O U N D}

The prevalence of overweight and obese children and adolescents has increased throughout the world, presenting a global public health crisis ( $\mathrm{Ng}$ 2014; WHO 2015a). Although once considered to be a condition affecting only developed countries, rates of paediatric overweight and obesity have started to rise dramatically in some developing countries (Wang 2012). Using the International Obesity Task Force (IOTF) standard definition, the age-standardised prevalence of overweight and obesity in children and adolescents has increased in both developed and developing countries since the mid-1980s (Cole 2000). In 2013, the prevalence of overweight and obese children and adolescents in developed countries was estimated at $23.8 \%$ (95\% confidence interval (CI) 22.9 to 24.7)) for boys and $22.6 \%$ (95\% CI 21.7 to 23.6) for girls. In developing countries, the prevalence was estimated as $12.9 \%(95 \%$ CI 12.3 to 13.5 ) for boys and $13.4 \%$ (95\% CI 13.0 to 13.9$)$ for girls ( $\mathrm{Ng} 2014)$. Very young children are also affected. In 2010, de Onis 2010 used the World Health Organization (WHO) growth standards (WHO 2015b) to estimate that over 42 million children under five years of age were overweight or obese, with approximately 35 million of these children living in developing countries.

Inequalities in overweight and obesity prevalence have also been documented. Generally, socioeconomically disadvantaged children in developed countries (Knai 2012; Shrewsbury 2008), and children of higher socioeconomic status in developing countries (Lobstein 2004; Wang 2012), are at greater risk of becoming overweight. However, this relationship may vary by population demographics (e.g. age, gender, ethnicity), and environment (e.g. country, urbanisation) (Wang 2012). The prevalence of obesity varies by ethnicity, with large data sets showing substantial ethnic variation in English (HSCIC 2015), American (Freedman 2006; Skinner 2014), and New Zealand (Rajput 2014) child populations.

While there is some evidence that the rate of increase in paediatric obesity may be slowing in some developed countries, current levels remain too high, and continue to rise in many developing countries (Olds 2011; Rokholm 2010). However, an additional concern in some developed countries such as the USA (Kelly 2013; Skinner 2014), and England (CMO 2012; Ells 2015), is the rise in severe paediatric obesity. While the IOTF published an international definition for severe paediatric (morbid) obesity in 2012 (Cole 2012), often severe obesity prevalence is reported using countryspecific cut-off points making international comparisons difficult. However, data from the USA (Skinner 2014), and England (Ells 2015), have shown that the prevalence of severe paediatric obesity varies by socioeconomic status and ethnicity, and may result in a greater risk of adverse cardio-metabolic events and severe obesity in adulthood (Kelly 2013).

\section{Description of the condition}

Childhood overweight and obesity results from an accumulation of excess body fat, and can increase the risk of both short- and longer-term health consequences. Numerous obesity-related comorbidities can develop during childhood, which include muscular skeletal complaints (Paulis 2014); cardiovascular risk factors such as hypertension, insulin resistance and hyperlipidaemia (Reilly 2003), even in very young children (Bocca 2013); and conditions such as such as sleep apnoea (Narang 2012), asthma (Egan 2013), liver disease, and type 2 diabetes (Daniels 2009; Lobstein 2004). The condition can also affect psychosocial wellbeing, with obese young people susceptible to reduced self esteem and health-related quality of life (Griffiths 2010), and stigmatisation (Puhl 2007; Tang-Peronard 2008). Evidence also shows that childhood obesity can track into adulthood (Parsons 1999; Singh 2008; Whitaker 1997), and is therefore associated with an increased risk of ill health later in life (Reilly 2011).

\section{Description of the intervention}

Given the serious implications associated with childhood and adolescent obesity, effective treatment is imperative. While the fundamental principles of weight management in children and adolescents are the same as adults (i.e. reduced energy intake and increased energy expenditure), the primary aim of treatment (i.e. weight reduction or deceleration of weight gain) and the most suitable intervention approach varies, and is dependent on the child's age and degree of excess weight, among other considerations. Family-based interventions combining dietary, physical activity and behavioural components are effective and are considered as the current best practice in the treatment of childhood obesity in children under 12 years of age (Oude Luttikhuis 2009). However, interventions that involve the whole family can be costly, especially with parents and children in separate groups and when not running at full capacity (Upton 2012). Therefore, increased attention is being paid to the possibility of parent-focused interventions.

Parents have been defined as the 'agents of change' for intervening with children under 12 years of age who are obese (Golan 2004). Several interventions have been developed where parents are targeted solely for the treatment of their child's obesity, thereafter referred to as 'parent-only' interventions, in which the child is not involved directly with the intervention. Parent-only interventions vary both by type (e.g. based on parenting courses, cognitive behavioural therapy, behaviour change) and by setting (e.g. community, clinic based).

\section{Adverse effects of the intervention}

It is not anticipated that parent-only interventions will lead to adverse outcomes. However, as with all obesity treatment interventions in children and young people, potential adverse effects 
should be considered, including effects on linear growth, eating disorders and psychological well-being.

\section{How the intervention might work}

The home environment is important in the aetiology of childhood obesity, with parents playing a large role in food choice and physical activity for their children. In surveys in the US, Wansink estimated that the 'nutritional gatekeeper' (who buys and cooks the food) controls $72 \%$ of the food eaten by children, both within and outside the home (Wansink 2006). One systematic review by Clark et al. showed that a high level of parental restriction of snack foods is associated with increased energy intake and weight gain in children (Clark 2007). In contrast, 'covert' control of children's food intake by controlling the home eating environment to limit exposure to unhealthy foods (i.e. not buying unhealthy foods) lows the intake of unhealthy snacks when compared with 'overt' control (i.e. buying the snacks but not allowing access) (Ogden 2006). In terms of physical activity, one systematic review showed that parental support is strongly associated with physical activity levels in children, albeit the influence of parental modelling by being physically active themselves was inconsistent (Gustafson 2006). Poor family functioning, such as poor communication and high levels of conflict, is also associated with higher risk of obesity in children (Halliday 2014). Authoritative parenting style is associated with lower risk of obesity in children, when compared with other parenting styles (Sleddens 2011).

Due to the importance of the role of parents in the home environment and the importance of parenting styles and skills, parents have been defined as the 'agents of change' in the family for intervening with children under 12 years of age who are obese (Golan 2004). The importance of parents in the change process has led to a questioning of whether children need to be at the intervention. Parent-only interventions aim to work by giving parents the responsibility for their family's eating and physical activity environment and by increasing parental capacity to implement the lifestyle changes. Trials have assessed whether parent-only interventions are superior to or equivalent to parent-child interventions, as well as comparisons with waiting list control.

\section{Why it is important to do this review}

The first version of this systematic review was published in 2003 and included analysis of childhood obesity treatment trials published up to July 2001 (Summerbell 2003). The second version was published in 2009 providing an update to the 2003 review (Oude Luttikhuis 2009).

To reflect the rapid growth in this field, the third update to this review has been split across six reviews focusing on the following treatment approaches: surgery; drugs; parent-only interventions; diet, physical activity and behavioural interventions for young children aged 0 to 4 years; school children aged 5 to 11 years and adolescents aged 12 to 17 years.

The current review examined the effectiveness of interventions in which parents were targeted solely for the treatment of childhood obesity. This review built on two reviews in this area in which parent-only interventions appeared to be as effective as interventions that adopted the traditional model where the parent and child were both involved in the intervention (Ewald 2014; Jull 2013). Faith 2012 revealed inconsistent evidence that greater parent and adult carer involvement was associated with better child outcomes. This review extended the evidence of effectiveness by including trials that compared parent-only interventions with parent-child interventions, waiting list controls, other interventions with only minimal information or contact and other types of parent-only interventions. These trials were brought together to examine the effectiveness of parent-only interventions for the treatment of childhood obesity. The review also intended to explore the impact of the type of parent-only intervention (e.g. focusing on parenting, cognitive behavioural therapy, behaviour change) and the setting (e.g. community, clinic-based, internet), to determine if any specific approach was more effective for the treatment of childhood obesity.

The results of this current review and other systematic reviews in this series provided information on which to underpin clinical guidelines and health policy on the treatment of childhood overweight or obesity.

\section{O B J E C T I VES}

To assess the efficacy of diet, physical activity and behavioural interventions delivered to parents only for the treatment of overweight and obesity in children aged 5 to 11 years.

\section{METHODS}

\section{Criteria for considering studies for this review}

\section{Types of studies}

We included randomised controlled trials (RCTs).

\section{Types of participants}

Study groups consisted of children with a mean study age of 5 to 11 years at the commencement of the intervention.

Diagnostic criteria

We included overweight or obese children by any classification. 


\section{Types of interventions}

We planned to investigate the following comparisons of intervention versus control/comparator.

\section{Intervention}

Any form of lifestyle intervention with a primary aim to treat overweight or obesity in children (any form of dietary, physical activity, behavioural therapy, or a combination of these delivered as single or multi-component interventions) directed at the parents as the agents of change (i.e. interventions did not include their children).

\section{Comparator}

Usual care, a parent-child intervention, child only intervention or an alternative concomitant therapy providing it was delivered in the intervention arm.

Concomitant interventions had to be the same in the intervention and comparator groups to establish fair comparisons.

Minimum duration of intervention/follow-up

Duration of intervention/follow-up had to be at least six months. Exclusion criteria

We excluded critically ill children or children with a syndromic cause for their obesity (e.g. Prader-Willi).

\section{Types of outcome measures}

\section{Primary outcomes}

- Changes in body mass index (BMI) and body weight.

- Adverse events.

\section{Secondary outcomes}

- Health-related quality of life and self esteem.

- All-cause mortality.

- Morbidity.

- Measures of body fat distribution.

- Behaviour change.

- Participants' views of the intervention.

- Parent-child relationship or assessment of parenting.

- Socioeconomic effects by validated measures.

Method and timing of outcome measurement

- Changes in BMI $\left(\mathrm{kg} / \mathrm{m}^{2}\right)$ and body weight $(\mathrm{kg})$ measured at baseline, and at least at 6,12 and 24 months.

- Adverse events: defined as an adverse outcome that occurred during or after the intervention but was not necessarily caused by it, and measured at baseline, and at least at 6,12 and 24 months.

- Health-related quality of life: evaluated by a validated instruments such as the Paediatric Quality of Life Inventory and measured at baseline, and at least at 6,12 and 24 months.

- All-cause mortality: defined as any death that occurred during or after the intervention and measured at baseline, and at least at 6,12 and 24 months
- Morbidity: defined as illness or harm associated with the intervention and measured at baseline, and at least at 6,12 and 24 months.

- Measures of body fat distribution: defined using validated tools such as dual-energy X-ray absorptiometry (DXA), waist circumference, skin-fold thickness, waist-to-hip ratio or bioelectrical impedance analysis and measured at baseline, and at least at 6, 12 and 24 months.

- Behaviour change: defined as validated measures of diet and physical activity and measured at baseline, and at least at 6, 12 and 24 months.

- Participants' views of the intervention: defined as documents accounts from participant feedback and measured at baseline, and at least at 6, 12 and 24 months.

- Parent-child relationship or assessment of parenting: evaluated by a validated instrument and measured at baseline, and at least at 6,12 and 24 months.

- Socioeconomic effects defined as a validated measure of socioeconomic status such as parental income or educational status and measured at baseline, and at least at 6,12 and 24 months.

\section{Summary of findings}

We present a 'Summary of findings' table to report the following outcomes, listed according to priority.

1. Changes in BMI and body weight.

2. Adverse events.

3. Health-related quality of life

4. All-cause mortality.

5. Mobidity.

6. Parent-dhild relationship or assessment of parenting.

7. Socioeconomic effects.

\section{Search methods for identification of studies}

\section{Electronic searches}

We searched the following sources from inception of each database to the specified date and placed no restrictions on the language of publication.

- Cochrane Library:

- Cochrane Database of Systematic Reviews (CDSR)

(Issue 3, 10 March 2015).

- Cochrane Central Register of Controlled Trials

(CENTRAL) (Issue 3, 10 March 2015).

- Database of Abstracts of Reviews of Effects (DARE)

(Issue 1, 10 March 2015).

- Health Technology Assessment (HTA) (Issue 1, 10

March 2015).

- MEDLINE and MEDLINE In-Process \& Other NonIndexed Citations, 1946 to 10 March 2015. 
- EMBASE, 1974 to 10 March 2015.

- PsycINFO, 1806 to 10 March 2015.

- CINAHL (10 March 2015).

- LILACS (10 March 2015).

- ClinicalTrials.gov (10 March 2015).

- World Health Organization (WHO) International Clinical Trials Registry Platform (ICTRP) Search Portal (http:// apps.who.int/trialsearch/), which is a meta-register of trials with links to several trial registers, that includes

- Australian New Zealand Clinical Trials Registry (2

March 2015).

- Chinese Clinical Trial Registry (2 March 2015).

- ClinicalTrials.gov (2 March 2015).

- EU Clinical Trials Register (EU-CTR) (2 March 2015).

- ISRCTN (International Standard Randomised Controlled Trial Number) Register (2 March 2015).

- The Netherlands National Trial Register (2 March 2015).

- Brazilian Clinical Trials Registry (2 February 2015).

- Clinical Trials Registry - India (2 March 2015).

- Clinical Research Information Service - Republic of Korea (3 March 2015).

- Cuban Public Registry of Clinical Trials (3 March 2015).

- German Clinical Trials Register (3 March 2015).

- Iranian Registry of Clinical Trials (3 March 2015).

- Japan Primary Registries Network (3 March 2015).

- Pan African Clinical Trial Registry (3 March 2015).

- Sri Lanka Clinical Trials Registry (2 March 2015).

- Thai Clinical Trials Register (3 March 2015).

We continuously applied a MEDLINE (via Ovid) email alert service established by the Cochrane Metabolic and Endocrine Disorders (CMED) Group to identify newly published trials using the same search strategy as described for MEDLINE (for details on search strategies see Appendix 1). Should we have identified new trials for inclusion, we would have evaluated these, incorporated findings in our review and re-submitted another review draft (Beller 2013).

\section{Searching other resources}

We tried to identify other potentially eligible trials or ancillary publications by searching the reference lists of retrieved included trials, (systematic) reviews, meta-analyses and HTA reports.

\section{Data collection and analysis}

\section{Selection of studies}

Two review authors (two of RJ, LA, KR, EL, JC, WR, EM) independently scanned the abstract, title, or both, of every record retrieved, to determine which trials should be assessed further. We investigated all potentially relevant articles as full text. We resolved any discrepancies through consensus or recourse to a third review author (KR, EL, LA). Where resolution of a disagreement was not possible, we added the article to those 'awaiting assessment' and contacted study authors for clarification. We presented an adapted PRISMA (Preferred Reporting Items for Systematic Reviews and Meta-Analyses) flow diagram showing the process of study selection (Liberati 2009).

\section{Data extraction and management}

For trials that fulfilled inclusion criteria, two review authors (two of RJ, LA, EL, JC, WR) independently abstracted key participant and intervention characteristics and reported data on efficacy outcomes and adverse events using standard data extraction templates as supplied by the CMED group, with any disagreements resolved by discussion, or, if required, by consultation with a third review author (KR) (for details see Table 1; Appendix 2; Appendix 3; Appendix 4; Appendix 5; Appendix 6; Appendix 7; Appendix 8; Appendix 9).

We provided information including trial identifier about potentially relevant ongoing trials in the Characteristics of ongoing studies table and in the 'Matrix of study endpoints (publications and trial documents)' where available. We tried to find the protocol of each included study in trial registers or publications of study designs, or both, and reported primary, secondary and other outcomes in comparison with data in publications in Appendix 5. We emailed all authors of included trials to enquire whether they were willing to answer questions regarding their trials. Appendix 10 shows the results of this survey. Thereafter, we sought relevant missing information on the trial from the primary author(s) of the article, if required.

\section{Dealing with duplicate and companion publications}

In the event of duplicate publications, companion documents or multiple reports of a primary study, we tried to maximise yield of information by collating all available data and use the most complete data set aggregated across all known publications. In case of doubt, we gave priority to the publication reporting the longest follow-up associated with our primary or secondary outcomes.

\section{Assessment of risk of bias in included studies}

Two review authors (two of RJ, LA, EL, JC) assessed the risk of bias of each included study independently. We resolved possible 
disagreements by consensus, or with consultation with a third review author (KR).

We used Cochrane's tool for assessing risk of bias (Higgins 2011a;

Higgins 2011b), and evaluated the following criteria.

- Random sequence generation (selection bias).

- Allocation concealment (selection bias).

- Blinding of participants and personnel (performance bias).

- Blinding of outcome assessment (detection bias).

- Incomplete outcome data (attrition bias).

- Selective reporting (reporting bias).

- Other potential sources of bias.

We judged the above risk of bias criteria as 'low risk', 'high risk' or 'unclear risk' and evaluated individual bias items as described in the Cochrane Handbook for Systematic Reviews of Interventions (Higgins 2011a). We presented a 'Risk of bias' graph and a 'Risk of bias' summary. We assessed the impact of individual bias domains on study results at endpoint and study levels. In case of high risk of selection bias, we marked all endpoints investigated in the associated study as high risk.

We evaluated whether imbalances in baseline characteristics existed and how these were addressed (Egbewale 2014).

For performance bias (blinding of participants and personnel) and detection bias (blinding of outcome assessors), we evaluated the risk of bias separately for each outcome type (objective and subjective) (Hróbjartsson 2013).

We considered the implications of missing outcome data from individual participants per outcome such as high drop-out rates (e.g. above $15 \%$ ) or disparate attrition rates (e.g. difference of $10 \%$ or more between study arms).

We assessed outcome reporting bias by integrating the results of 'Examination of outcome reporting bias' (Appendix 6) and the 'Matrix of study endpoints (publications and trial documents)' (Appendix 5) (Kirkham 2010). This analysis formed the basis for the judgement of selective reporting (reporting bias).

We defined the following as self reported outcomes ('subjective outcomes').

- Adverse events.

- Health-related quality of life and self esteem.

- Parent-child relationship or assessment of parenting.

- Participants' views of the intervention.

We defined the following as investigator-assessed outcomes ('objective outcomes').

- Changes in BMI measures and body weight.

- Measures of body fat distribution.

- Adverse events.

- All-cause mortality.

- Morbidity.

- Behaviour change.

- Socioeconomic effects.

\section{Measures of treatment effect}

We expressed dichotomous data as odds ratios (ORs) or risk ratios (RRs) with 95\% confidence intervals (CIs). We expressed continuous data as mean differences (MD) with $95 \%$ CI. We planned to express time-to-event data as hazard ratios (HRs) with 95\% CIs.

\section{Unit of analysis issues}

We took into account the level at which randomisation occurred, such as cross-over trials, cluster-randomised trials and multiple observations for the same outcome. For cluster-randomised trials, we used the adjusted data reported in the original studies. Where studies had multiple control groups, we used data from the control group for each comparison by reducing the weight assigned to the control group by dividing the number of participants in the control group by the number of intervention groups.

\section{Dealing with missing data}

We obtained relevant missing data from authors, if possible, and evaluated important numerical data such as screened, eligible, randomised participants as well as intention-to-treat (ITT), as-treated and per-protocol (PP) populations where possible. We investigated attrition rates, for example drop-outs, losses to follow-up and withdrawals, and critically appraise issues of missing data and imputation methods (e.g. last-observation-carried-forward (LOCF)). Where standard deviations (SD) for outcomes were not reported, and we did not receive information from study authors, we calculated these following the methods presented in the Cochrane Handbook for Systematic Reviews of Interventions (Higgins 2011a). Where papers did not report results as change from baseline, we calculated this and for the SD differences followed the methods presented in the Cochrane Handbook for Systematic Reviews of Interventions for imputing these (Section 16.1.3.2 Imputing standard deviations for changes from baseline; Higgins 2011a), and assumed a correlation of 0.5 between baseline and follow-up measures as suggested by Follman 1992.

\section{Assessment of heterogeneity}

In the event of substantial clinical or methodological heterogeneity, we did not report study results as meta-analytically pooled effect estimates. We identified heterogeneity by visual inspection of the forest plots and by using a standard $\mathrm{Chi}^{2}$ test with a significance level of $\alpha=0.1$, in view of the low power of this test. We examined heterogeneity using the $\mathrm{I}^{2}$ statistic, which quantifies inconsistency across trials to assess the impact of heterogeneity on the meta-analysis (Higgins 2002; Higgins 2003), where an $\mathrm{I}^{2}$ statistic of $75 \%$ or more indicates a considerable level of inconsistency (Higgins 2011a).

When we found heterogeneity, we attempted to determine potential reasons for it by examining individual study and subgroup characteristics. 


\section{Assessment of reporting biases}

If we had included 10 trials or more for a given outcome, we would have used funnel plots to assess small-study effects. Due to several explanations for funnel plot asymmetry, we would have interpreted results carefully (Sterne 2011).

\section{Data synthesis}

Unless there was good evidence for homogeneous effects across trials, we primarily summarised data by means of a random-effects model (Wood 2008). We had planned to interpret randomeffects meta-analyses with due consideration of the whole distribution of effects, ideally by presenting a prediction interval; however, there were relatively few trials included in each category, of low methodological quality and so theses analyses were not conducted (Higgins 2009). A prediction interval specifies a predicted range for the true treatment effect in an individual study (Riley 2011). We performed statistical analyses according to the statistical guidelines referenced in the latest version of the Cochrane Handbook for Systematic Reviews of Interventions (Higgins 2011a).

\section{Quality of evidence}

We present the overall quality of the evidence for each outcome according to the Grading of Recommendations Assessment, Development and Evaluation (GRADE) approach, which takes into account issues not only related to internal validity (risk of bias, inconsistency, imprecision, publication bias) but also to external validity such as directness of results. Two review authors (EL, KR) rated the quality for each outcome. We presented a summaries of the evidence in a 'Summary of findings' tables, which provide key information about the best estimate of the magnitude of the effect, in relative terms and absolute differences for each relevant comparison of alternative management strategies, numbers of participants and trials addressing each important outcome, and the rating of the overall confidence in effect estimates for each outcome. We created the 'Summary of findings' tables based on the methods described in the Cochrane Handbook for Systematic Reviews of Interventions (Higgins 2011a). We presented results on the outcomes as described in Types of outcome measures.

In addition, we established an appendix 'Checklist to aid consistency and reproducibility of GRADE assessments' (Meader 2014) to help with standardisation of 'Summary of findings' tables (Appendix 9).

\section{Subgroup analysis and investigation of heterogeneity}

We expected the following characteristics to introduce clinical heterogeneity, and aimed to carry out subgroup analyses with investigation of interactions where data permitted.

- Differences in BMI at baseline.

- Length of follow-up.

- The impact of comparator/control: whether concomitant therapy or no treatment (true control).

- The setting in which the intervention was conducted.

\section{Sensitivity analysis}

We planned to perform sensitivity analyses to explore the influence of the following factors on effect size.

- Restricting the analysis to published trials.

- Restricting the analysis taking into account risk of bias, as specified in the 'Assessment of risk of bias in included studies' section.

- Restricting the analysis to very long or large trials to establish how much they dominated the results.

- Restricting the analysis to trials using the following filters: diagnostic criteria, language of publication, source of funding (industry versus other), country.

We tested the robustness of the results by repeating the analysis using different statistical models (fixed-effect and random-effects models).

\section{RE S U L T S}

\section{Description of studies}

For a detailed description of trials, see the Characteristics of included studies, Characteristics of excluded studies and Characteristics of ongoing studies tables.

\section{Results of the search}

The searches generated 13,759 hits after duplicates were removed. Screening of titles and abstracts identified 137 papers to go forward for formal inclusion and exclusion. Twenty completed RCTs fulfilled the inclusion criteria and were included in the review. For a detailed description of the included trials, see the Characteristics of included studies table. The search identified 10 ongoing trials, which are reported in the Characteristics of ongoing studies table. The flow of trials through the review is presented in Figure 1. 
Figure I. Study flow diagram.

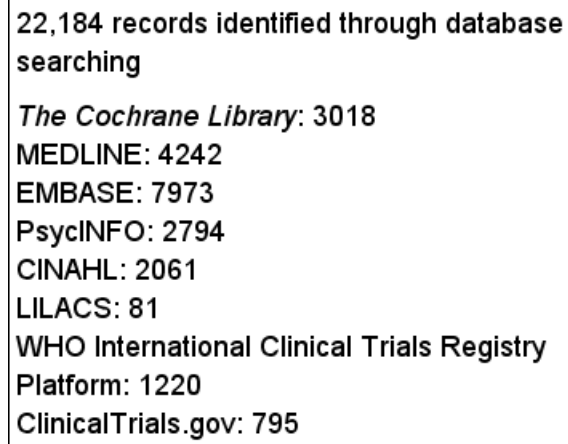

10 additional records identified through non-database sources (contacts with experts, manufacturers, handsearching of literature)

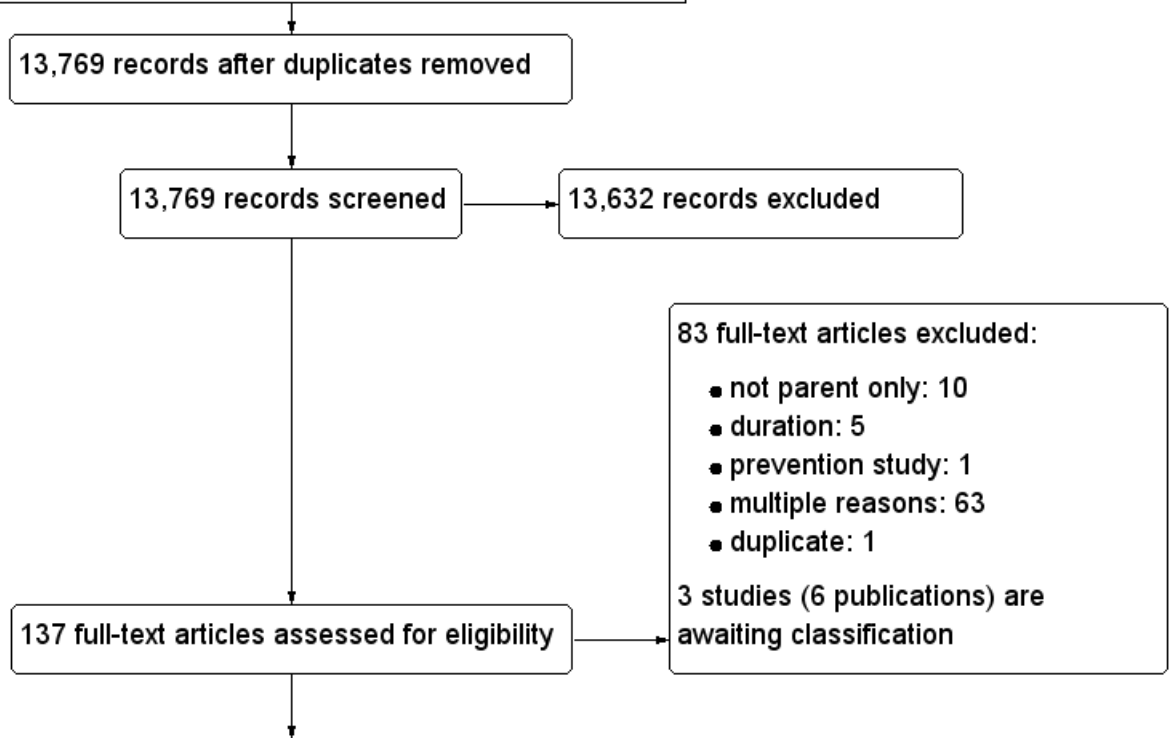

0 additional studies identified through handsearching of reference lists of included trials, systematic reviews/meta-analyses and HTA reports

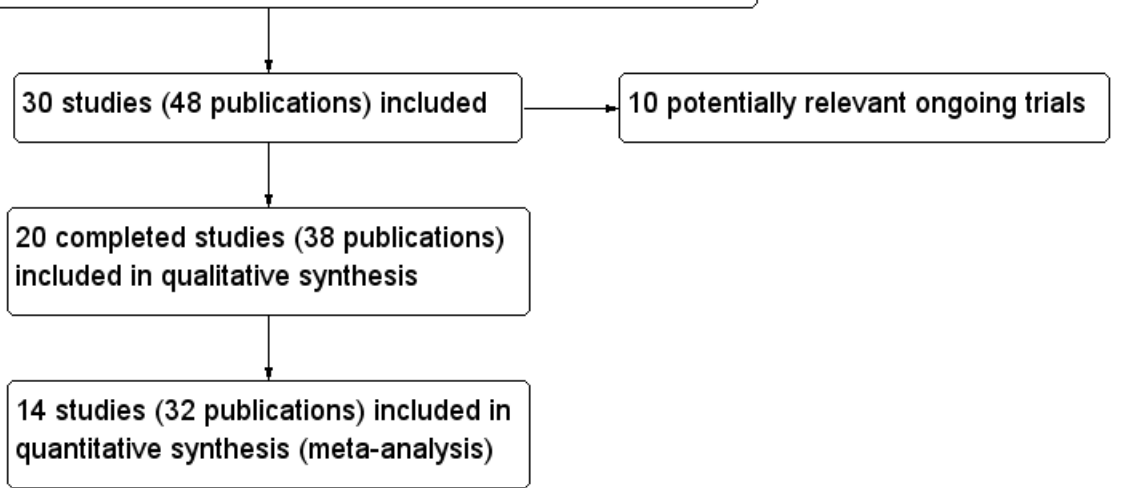




\section{Included studies}

A detailed description of the characteristics of included trials is presented elsewhere (see Characteristics of included studies table and Appendix 2; Appendix 3; Appendix 4; Appendix 5; Appendix 6; Appendix 7; Appendix 8; Appendix 9). The following is a succinct overview.

\section{Source of data}

The majority of data presented in the review was obtained from published literature, including supplementary published data where available. For five trials, trial authors provided data (Appendix 10).

\section{Study details}

We included 20 RCTs in 19 main publications (Aragona 1975; Boutelle 2011; Collins 2011; Esfarjani 2013; Estabrooks 2009; Golan 2006; Golley 2007; Janicke 2008; Jansen 2011; Magarey 2011; Mazzeo 2014; Munsch 2008; Raynor 2012a; Raynor 2012b; Resnick 2009; Resnicow 2015; Small 2013; van Grieken 2013; West 2010). Eighteen RCTs were parallel comparisons with individual randomisation. In most trials, the unit of randomisation was the family (parent and child); however, study authors analysed the children and parents for respective outcomes separately. Two RCTs were cluster RCTs, where the Primary Care Provider (PCP) (Resnicow 2015) or Youth Health Care (YHC) (van Grieken 2013) team were the unit of randomisation. Eighteen RCTs were superiority trials, one had a non-inferiority study design (Boutelle 2011), and one an equivalence study design (Munsch 2008). Eight RCTs had three comparisons (Aragona 1975; Collins 2011; Estabrooks 2009; Golley 2007; Janicke 2008; Raynor 2012a; Raynor 2012b; Resnicow 2015); the remaining trials had two comparison groups. Ten trials were undertaken in the USA (Aragona 1975; Boutelle 2011; Estabrooks 2009; Janicke 2008; Mazzeo 2014; Raynor 2012a; Raynor 2012b; Resnick 2009; Resnicow 2015; Small 2013); four in Australia (Collins 2011; Golley 2007; Magarey 2011; West 2010), and two in the Netherlands (Jansen 2011; van Grieken 2013). There was one trial each from Israel (Golan 2006), Switzerland (Munsch 2008), Iran (Esfarjani 2013), and Belgium (Moens 2012). Five trials were single-centre trials (Aragona 1975; Boutelle 2011; Esfarjani 2013; Golan 2006; Moens 2012); the remaining trials were either multi-centre trials (with two centres: Golley 2007; Munsch 2008; Resnick 2009; three centres: Jansen 2011; Magarey 2011; and six centres: West 2010), or the numbers of centres were not reported (Collins 2011; Estabrooks 2009; Janicke 2008; Mazzeo 2014; Raynor 2012a; Raynor 2012b; Small 2013). The cluster RCT by van Grieken 2013 was undertaken in nine study centres, across 44 healthcare teams. The cluster RCT by Resnicow 2015 was undertaken in 42 primary care practices.

\section{Overview of study populations}

All trials included parents of overweight or obese children. The diagnostic criteria differed between trials, in 10 trials this was based on the BMI 85th percentile (Boutelle 2011; Estabrooks 2009; Golan 2006; Janicke 2008; Mazzeo 2014; Munsch 2008; Raynor 2012a; Raynor 2012b; Resnick 2009; Resnicow 2015), and in one trial the BMI 95th percentile (Esfarjani 2013). In other trials, this was based on the parent or physician describing their child as overweight (Aragona 1975; Moens 2012; Small 2013; West 2010); the IOTF definition (Golley 2007; Magarey 2011), or stated as international cut-off points that were not specified (van Grieken 2013); a specified BMI cut-off (Collins 2011); or the proportion of BMI above expected BMI (at least 30\% greater in Jansen 2011). All trials included parents of children aged between 4 and 13 years, the majority of which did not include children above 11 years of age. The mean ages of participants were reported in 16 trials. In six trials, the mean ages were between 5 and 7 years (Esfarjani 2013; Raynor 2012a; Raynor 2012b; Resnicow 2015; Small 2013; van Grieken 2013); in seven trials, the mean ages were between 8 and 9 years (Aragona 1975; Collins 2011; Golan 2006; Magarey 2011; Moens 2012; West 2010); and in four trials, the mean ages of the children was between 10 and 11 years (Boutelle 2011; Estabrooks 2009; Janicke 2008; Munsch 2008). The proportion of girls in the trials typically ranged from $40 \%$ to $70 \%$ where reported (four trials did not report this: Esfarjani 2013; Janicke 2008; Mazzeo 2014; Resnick 2009), although was $100 \%$ in one study (Aragona 1975). Only eight trials reported ethnicity of the children and in all trials there was a high proportion of children categorised as white (between $60 \%$ and $100 \%$ across all trials: Estabrooks 2009; Janicke 2008; Moens 2012; Raynor 2012a; Raynor 2012b; Resnicow 2015; Small 2013; West 2010). Only eight trials reported socioeconomic indices of the parents (Boutelle 2011; Esfarjani 2013; Janicke 2008; Moens 2012; Resnicow 2015; Small 2013; van Grieken 2013; West 2010). Each used a different indicator of socioeconomic status (see Appendix 4).

The number of participants included in the 19 trials ranged between 15 and 645. Five trials had fewer than 30 participants per study arm (Aragona 1975; Moens 2012; Munsch 2008; Raynor 2012b; Resnick 2009), whereas four trials had greater than 60 participants per study arm (Collins 2011; Esfarjani 2013; Estabrooks 2009; Magarey 2011). In the cluster RCT by van Grieken 2013, there were 22 clusters in each arm (total 637 participants), in the Resnicow 2015 cluster RCT, there were 42 clusters (total 645 participants).

Seven trials reported the BMI z score, which ranged from 2.0 to

Parent-only interventions for childhood overweight or obesity in children aged 5 to II years (Review) 
2.8 at baseline (Boutelle 2011; Collins 2011; Estabrooks 2009; Golley 2007; Janicke 2008; Magarey 2011; West 2010). Five trials reported the BMI percentile, which ranged from $92 \%$ to $98.5 \%$ at baseline (Jansen 2011; Mazzeo 2014; Resnick 2009; Resnicow 2015; Small 2013). Five trials reported BMI (Esfarjani 2013; Mazzeo 2014; Munsch 2008; Raynor 2012a; Raynor 2012b), and could be calculated from individual participant data in one other study (Aragona 1975). The BMI in these trials ranged from 22 to 33.6 at baseline. Eleven trials reported parental BMI. In eight trials, the mean parental BMI ranged between 26 and 36; with four trials including parents with BMIs between 26 and 30 (Jansen 2011; Munsch 2008; Resnick 2009; Resnicow 2015), and four trials including parents with BMIs above 30 (Boutelle 2011; Janicke 2008; Mazzeo 2014; Small 2013). One study reported the proportions of parents in three categories (healthy, overweight, obese); these were approximately 37\% (healthy), 23\% (overweight) and $40 \%$ (obese) (West 2010). Another study reported the proportions of 'normal' (about 56\%) and 'overweight' (about 44\%) (van Grieken 2013). One study reported the weight of the parents (for mothers and fathers for each group respectively) (Golan 2006). For details of baseline characteristics of participants in the included trials, see Appendix 4 and Appendix 5. There were no substantial differences in baseline characteristics between the intervention and comparator groups in the included trials.

\section{Interventions}

Seventeen trials reported the settings for the interventions. In four trials, the intervention was in an outpatient setting (Collins 2011; Estabrooks 2009; Golley 2007; Magarey 2011), and in four trials it was a community setting (Janicke 2008; Mazzeo 2014; Resnick 2009; van Grieken 2013). Two trials were undertaken in a university setting (Boutelle 2011; Moens 2012); two in a primary care setting (Resnicow 2015; Small 2013), and five trials win a mixture of settings including outpatient, university, primary care or a combination of these settings (Jansen 2011; Munsch 2008; Raynor 2012a; Raynor 2012b; West 2010).

The interventions in the included trials predominantly focused on nutritional, physical activity and behavioural components; see Characteristics of included studies table and Appendix 2 for specific details for each included study.

Five trials compared a parent-only intervention to a parent-child intervention (two also had a third comparison of a waiting list control, Janicke 2008 or a second parent-child intervention, Collins 2011) (Boutelle 2011; Collins 2011; Golan 2006; Janicke 2008; Munsch 2008). In one study, the duration of the intervention was 10 weeks (Munsch 2008); and four trials had interventions of 16 to 24 weeks' duration (Boutelle 2011; Collins 2011; Golan 2006; Janicke 2008). Session lengths ranged from one to two hours in all five trials. All trials followed participants beyond the timing of the end of the intervention. This was 14 weeks' post intervention in one study (Janicke 2008), 24 to 26 weeks' post intervention in two trials (Boutelle 2011; Munsch 2008), one year post intervention in one trial (Golan 2006), and 80 weeks' post intervention in one study (Collins 2011).

Six trials compared a parent-only intervention to a waiting list control (Aragona 1975; Golley 2007; Janicke 2008; Jansen 2011; Moens 2012; West 2010) and two of these trials had two different parent-only interventions (Aragona 1975; Golley 2007). The duration of the intervention ranged from 12 to 24 weeks in these trials and sessions ranged between 90 minutes and two hours (one study did not provide details, Aragona 1975). In two trials, there were additional sessions via telephone contact between interventionists and the parents, which were approximately $20 \mathrm{~min}$ utes long (Golley 2007; West 2010). All but one study followed participants up beyond the timing of the end of the intervention (Moens 2012). This was 12 to 14 weeks post intervention in one trial (Jansen 2011), 24 weeks post intervention in two trials (Golley 2007; Janicke 2008), 39 weeks post intervention in one trial (Aragona 1975) and 40 weeks post intervention in one trial West 2010).

Seven trials compared a parent-only intervention with a minimal contact parenting advice control (mailed information or a workbook or minimal sessions) (Esfarjani 2013; Estabrooks 2009; Mazzeo 2014; Resnick 2009; Resnicow 2015; Small 2013; van Grieken 2013); two trials had two different parent-only interventions (Estabrooks 2009; Resnicow 2015). The duration of the interventions ranged from 12 to 26 weeks in five trials (in the Resnick 2009 study the interventions ranged from 30 to 41 weeks but the study reports a mean of 18 weeks). In one study, the duration of the intervention differed for each participants, but was up to 12 months (van Grieken 2013). In one trial, the intervention continued to 24 months (Resnicow 2015). In three trials, the number of sessions provided to parents was lower than reported in the other parent-only interventions, being three or four sessions (Resnick 2009; Small 2013; van Grieken 2013). In two trials, the intervention was delivered across 12 sessions (Esfarjani 2013; Mazzeo 2014); in the study by Estabrooks 2009, there were two parentonly treatment arms, which were either 12 or 24 weeks in duration and in the study by Resnicow 2015, there were also two parentonly treatment arms, which were either four or 10 sessions. Six trials reported the duration of follow-up in relation to the completion of the intervention. In the study by Mazzeo 2014, this was 12 weeks post intervention completion, in Small 2013, this was 24 weeks post completion of the intervention, in Estabrooks 2009, this was between 28 and 40 weeks post completion of the intervention and in Aragona 1975, this was 39 weeks post completion of the intervention. In the van Grieken 2013 RCT, follow-up was 24 months after baseline (the duration of the intervention was "up to 12 months") and in the Resnicow 2015 trial, follow-up was at completion of the intervention at 24 months.

Seven trials compared different types of parent-only interventions (Aragona 1975; Estabrooks 2009; Golley 2007; Magarey 2011; Raynor 2012a; Raynor 2012b; Resnicow 2015), two also had a 
parent-only versus waiting list control comparison (Aragona 1975; Golley 2007), and two trials also had a parent-only versus minimal contact intervention (Estabrooks 2009; Resnicow 2015). In the first, by Estabrooks 2009, there were three treatment arms. In one treatment group, the parents received a self help workbook, attended two group sessions and these were followed up with 10 interactive voice response (IVR) counselling sessions over a 10week period. In the second arm, the parents received the work book and attended the same two group sessions but did not have the IVR follow-up sessions. In the third group, parents received the workbook only. In the study by Resnicow 2015, participants either received motivational interviewing over four sessions in the primary care practice, or received the four motivational interviewing sessions and six sessions with a dietician (the third arm received usual care information). In the study by Magarey 2011, parents were randomised into two arms, one received a four-session parenting programme that was followed by eight group sessions about healthy lifestyles. The second group received the eight sessions about healthy lifestyles only. Both the trials by Estabrooks 2009 and Magarey 2011 followed participants up beyond the timing of the end of the intervention. In the study by Estabrooks 2009, this was between 28 and 40 weeks post completion of the intervention and in the study by Magarey 2011, this was 80 weeks post completion of the intervention. In the study by Resnicow 2015, followup was at the point of completing the intervention (24 weeks) although in the second 12-month period of the intervention there were fewer sessions held.

Two trials in one publication also compared different parent-only interventions (Raynor 2012a; Raynor 2012b). There were many shared attributes of the two trials. The first trial compared a parent-only intervention that focused on growth monitoring to a parent intervention that focused on decreasing sugary foods in the diet and a parent intervention that focused on increasing healthy food consumption (Raynor 2012a). Parents randomised to the two comparison groups also received the parent-only intervention (growth monitoring). The second study compared the parent-only intervention to a parent intervention that focused on decreasing sugary foods and increasing physical activity, and to a parent intervention that focused on increasing low-fat milk intake and decreasing television watching (Raynor 2012b). Parents randomised to the two comparison groups also received the parentonly 'growth monitoring' intervention. The duration of the interventions in these two trials was six months, and there were eight sessions of 45 minutes' duration. These trials followed participants up 24 weeks after the timing of the end of the intervention. Golley 2007 compared parenting skills training with the addition of intensive lifestyle education with the parenting skills training without the additional intensive lifestyle education. The duration of the interventions are as described above. Aragona 1975 compared a parenting response-cost and training in reinforcement skills with the response-cost training only. Parents were required to enter a contract with the interventionists and pay a deposit relating to a goal of weight loss in their child, which could be returned for attendance at the sessions and when the goal was achieved. The duration of the interventions is as described above.

Six trials reported compliance rates with the interventions ( Estabrooks 2009; Golan 2006; Golley 2007; Mazzeo 2014; Raynor 2012a; Raynor 2012b). One study reported that attendance in both arms of their trial was above $80 \%$ (Golan 2006). In two trials, the trial authors reported that attendance at growth monitoring appointments did not differ among the groups (Raynor 2012a; Raynor 2012b). In Raynor 2012a, compliance with attendance and turning in monitoring dairies was $73 \%$, in Raynor 2012b this was $64 \%$. In the study by Mazzeo 2014, the numbers attending $50 \%$ or more of sessions were reported for the parent-only intervention (22/43). Mean session attendance across groups was $53 \%$. In the control group, $35 / 41$ parents attended the single session. Golley 2007 reported the number of participants attending sessions in the two intervention arms. There were 11 sessions in the parenting-skill training arm and 19 parents attended at least eight sessions; 13 attended 2 to 7 sessions and 5 attended 1 or 0 sessions. In the parenting-skills training with intensive lifestyle education arm, there were 18 sessions and the study reported that 18 parents attended at least 13 sessions; 18 attended 2 to 12 sessions; and 2 attended 1 or sessions. Estabrooks 2009 reported the number of participants taking part in the IVR intervention where 20 participants took part in 0 to 5 calls while 38 took part in 6 to 10 calls.

\section{Outcomes}

All trials reported weight, BMI, or both, as an outcome measure. Other outcomes reported in the trials differed with few similarities across the included trials in the choice of outcomes reported (see Appendix 5). Where reported, measures of BMI were reported differently across the trials. Where a study reported more than one measure of BMI, we took the BMI z score as the preferred measure in the data synthesis.

\section{Excluded studies}

We excluded 83 of 137 full-text articles after evaluation of the full publication.

The main reasons for exclusion were the interventions also included the children (not parent-only) and the duration of the study was less than six months. Many trials had multiple reasons for exclusion (for further details see Characteristics of excluded studies table, which lists the 16 trials that most closely missed the inclusion criteria).

\section{Risk of bias in included studies}

For details on risk of bias of included trials, see Characteristics of included studies table. For an overview of review authors' judge- 
ments about each risk of bias item for individual trials and across

all trials, see Figure 2 and Figure 3.

Figure 2. Risk of bias graph: review authors' judgements about each risk of bias item presented as percentages across all included studies (blank cells indicate that the particular outcome was not investigated in some studies).

\begin{tabular}{l} 
Random sequence generation (selection bias) \\
Blinding of participants and personnel (performance bias): Subjective outcomes \\
Blinding of participants and personnel (performance bias): Objective outcomes \\
Blinding of outcome assessment (detection bias): Subjective outcomes \\
Blinding of outcome assessment (detection bias): Objective outcome \\
Incomplete outcome data (attrition bias): Subjective outcomes \\
Incomplete outcome data (attrition bias): Objective outcomes \\
Selective reporting (reporting bias) \\
\hline Low risk of bias
\end{tabular}


Figure 3. Risk of bias summary: review authors' judgements about each risk of bias item for each included study (blank cells indicate that the study did not report that particular outcome).

\begin{tabular}{|c|c|c|c|c|c|c|c|c|c|c|}
\hline & 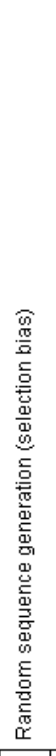 & 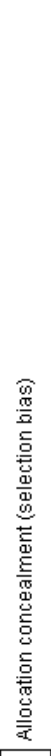 & 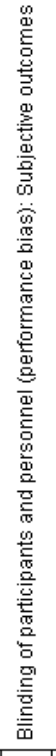 & 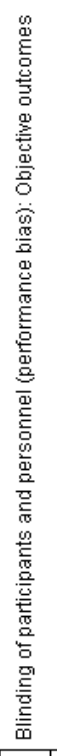 & 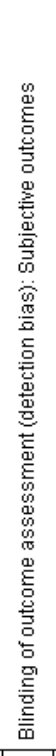 & 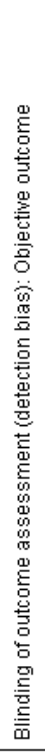 & 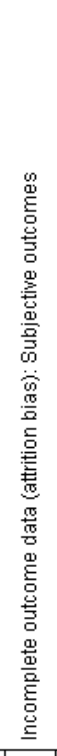 & 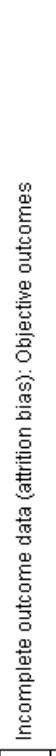 & 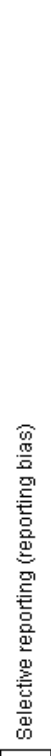 & 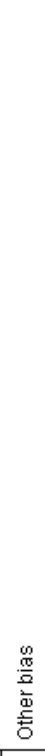 \\
\hline Aragona 1975 & $?$ & $?$ & & $?$ & & $?$ & & $\odot$ & $?$ & $?$ \\
\hline Boutelle 2011 & $\odot$ & $?$ & & $?$ & & $?$ & & $\odot$ & $?$ & $?$ \\
\hline Collins 2011 & 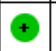 & $?$ & $\theta$ & $\theta$ & + & $\odot$ & $\theta$ & $\theta$ & $\theta$ & $?$ \\
\hline Esfarjani 2013 & $?$ & $?$ & & $?$ & & $?$ & & $\theta$ & $?$ & $?$ \\
\hline Estabrooks 2009 & $\odot$ & $?$ & $\theta$ & $\odot$ & $\theta$ & + & + & - & - & $?$ \\
\hline Golan 2006 & $?$ & + & $?$ & $?$ & $\odot$ & + & + & + & $?$ & $?$ \\
\hline Golley 2007 & $\odot$ & $\odot$ & & $?$ & & $\odot$ & & $?$ & 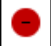 & $?$ \\
\hline Janicke 2008 & $?$ & $?$ & $\theta$ & 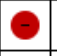 & $?$ & $?$ & - & $\theta$ & $\theta$ & $?$ \\
\hline Jansen 2011 & $?$ & $?$ & $?$ & $?$ & $?$ & $?$ & + & $\odot$ & $?$ & 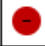 \\
\hline Magarey 2011 & $\odot$ & + & $\Theta$ & $\odot$ & $\odot$ & $\odot$ & - & - & $\Theta$ & $?$ \\
\hline Mazzeo 2014 & $\odot$ & $?$ & $?$ & $?$ & $?$ & $?$ & + & $\odot$ & $\odot$ & $?$ \\
\hline Moens 2012 & - & 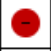 & - & - & $\Theta$ & $\Theta$ & $?$ & $?$ & $\theta$ & $?$ \\
\hline Munsch 2008 & $?$ & $?$ & $?$ & $?$ & $?$ & $?$ & - & 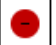 & $?$ & $?$ \\
\hline Raynor 2012a & $\odot$ & + & & $?$ & & $\odot$ & & $\odot$ & $?$ & $?$ \\
\hline Raynor 2012b & $\odot$ & $\odot$ & & $?$ & & $\odot$ & & + & $?$ & $?$ \\
\hline Resnick 2009 & $?$ & $?$ & & $?$ & & $?$ & & $\odot$ & $?$ & $?$ \\
\hline Resnicow 2015 & $?$ & $?$ & & $\odot$ & & $\odot$ & & $\odot$ & 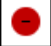 & $\odot$ \\
\hline Small 2013 & $?$ & $?$ & & $?$ & & $?$ & & + & $?$ & $?$ \\
\hline van Grieken 2013 & $\odot$ & $?$ & & $\odot$ & & $\odot$ & & $?$ & $\odot$ & $?$ \\
\hline West 2010 & $\odot$ & - & $\odot$ & $\odot$ & $\theta$ & $\Theta$ & + & $\odot$ & $?$ & $?$ \\
\hline
\end{tabular}


Trial quality was generally low. Many trials did not report adequate information to assess the risk of bias and we assessed 14 trials at high risk of bias on at least one domain (Boutelle 2011; Collins 2011; Esfarjani 2013; Estabrooks 2009; Golley 2007; Janicke 2008; Jansen 2011; Magarey 2011; Mazzeo 2014; Moens 2012; Munsch 2008; Resnick 2009; Resnicow 2015; West 2010). We assessed six trials at high risk of bias on three or more domains (Collins 2011; Estabrooks 2009; Janicke 2008; Magarey 2011; Moens 2012; West 2010).

\section{Allocation}

Only 10 of 19 trials reported an adequate method of randomisation (Boutelle 2011; Collins 2011; Estabrooks 2009; Golley 2007; Magarey 2011; Mazzeo 2014; Raynor 2012a; Raynor 2012b; van Grieken 2013; West 2010). Five trials reported an adequate concealment of allocation (Golan 2006; Golley 2007; Magarey 2011; Raynor 2012a; Raynor 2012b).

\section{Blinding}

The potential for performance bias was unknown in most trials. Six trials were at high risk of performance bias for objective or subjective (or both) outcomes as appropriate to their study outcomes (Collins 2011; Estabrooks 2009; Magarey 2011; Moens 2012; Resnicow 2015; West 2010). Blinding of outcome assessors was adequate for objective outcomes in nine trials (Collins 2011; Estabrooks 2009; Golan 2006; Golley 2007; Magarey 2011; Raynor 2012a; Raynor 2012b; Resnicow 2015; van Grieken 2013). In three trials, the risk of detection bias was high for subjective outcomes (Estabrooks 2009; Moens 2012; West 2010).

\section{Incomplete outcome data}

Many trials reported high levels of drop-out or loss to follow-up (highest drop-out rates ranged between $40 \%$ and $79 \%$ ) and only nine trials reported adequate means to address these in the analysis (Aragona 1975; Golan 2006; Jansen 2011; Mazzeo 2014; Raynor 2012a; Raynor 2012b; Resnicow 2015; Small 2013; West 2010). Eight trials were at high risk of attrition bias on objective outcomes (Boutelle 2011; Collins 2011; Esfarjani 2013; Estabrooks 2009; Janicke 2008; Magarey 2011; Munsch 2008; Resnick 2009), and four trials on subjective outcome measures (Collins 2011; Janicke 2008; Magarey 2011; Munsch 2008).

\section{Selective reporting}

One trial was at low risk of selective reporting bias (van Grieken 2013), whereas eight trials were at high risk of selective reporting bias (Collins 2011; Estabrooks 2009; Golley 2007; Janicke 2008; Magarey 2011; Mazzeo 2014; Moens 2012; Resnicow 2015). All other trials were at unclear risk of selective reporting bias.

\section{Other potential sources of bias}

One study by Jansen 2011 was at high risk of bias because nine participant families who had originally been randomised to the waiting list control were included in the analysis for the parentonly intervention. There were no other potential sources of bias identified by review authors and all of the remaining trials were at unclear risk of bias on this factor.

\section{Effects of interventions}

See: Summary of findings for the main comparison Parent-only interventions versus parent-child interventions for childhood overweight or obesity; Summary of findings 2 Parent-only interventions versus waiting list control for childhood overweight or obesity; Summary of findings 3 Parent-only interventions versus minimal contact control for childhood overweight or obesity; Summary of findings 4 Parent-only interventions versus parent-only interventions for childhood overweight or obesity The included trials had different durations of interventions and follow-up. To assess the effects of the interventions we considered outcomes from the longest period of follow-up in each study. In addition, we also reported outcomes from any post intervention follow-up period (if it differed from the longest period of followup). This allowed an assessment of the initial response to the intervention and any maintenance of that response on outcomes. If a study reported outcomes at any interim time points, we extracted these data as per the review protocol.

For 10 of 19 trials, we had to calculate SD as described (Dealing with missing data) (Boutelle 2011; Collins 2011; Esfarjani 2013; Estabrooks 2009; Jansen 2011; Magarey 2011; Mazzeo 2014; Resnick 2009; Small 2013; West 2010). Furthermore, one trial author provided SD data on two trials (Raynor 2012a; Raynor 2012b), after being contacted (Appendix 10).

\section{Parent-only interventions versus parent-child interventions}

Five trials reported seven comparisons of a parent-only intervention and a parent-child intervention (Boutelle 2011; Collins 2011; Golan 2006; Janicke 2008; Munsch 2008). To allow consideration of the effects of the interventions, we considered outcomes here from the longest period of follow-up and any post intervention follow-up. The period for the post intervention follow-up in these trials ranged from 10 weeks to 12 months and the period for the longest point of follow-up ranged from 8 to 24 months. Losses to follow-up ranged from $6 \%$ to $50 \%$ at the post intervention followup and $18 \%$ to $72 \%$ at the longest period of follow-up (see Table 1). One study reported the participants completing the study but it was unclear what numbers of participants were included in the

Parent-only interventions for childhood overweight or obesity in children aged 5 to II years (Review) 
post intervention follow-up (Golan 2006). In the trials by Golan 2006, Collins 2011, and Munsch 2008, there was a differential rate of losses to follow-up between groups. All of these factors need to be considered when interpreting the results of the trials.

\section{Primary outcomes}

\section{Changes in body mass index and body weight}

Five trials reported BMI variables at the end of the intervention (Boutelle 2011 at five months; Collins 2011 and Golan 2006 at six months; Janicke 2008 at four months; Munsch 2008 at 10 weeks). All trials reported the BMI z score. Three trials (four comparisons) reported data that could be analysed in a meta-analysis; Golan 2006 and Munsch 2008 did not report SDs or information that could be used to estimate SDs.

A pooled summary estimate of the change in $\mathrm{BMI} z$ score is shown in Analysis 1.1, the MD was -0.06 ( $(95 \%$ CI -0.13 to 0.02$)$; $\mathrm{P}$ $=0.14 ; 277$ participants; 3 trials with 4 treatment groups; low quality evidence). There was moderate heterogeneity $\left(\mathrm{I}^{2}=37 \%\right)$, and similar results occurred with a fixed-effect meta-analysis. All included trials had a high risk of attrition bias. SDs were imputed for one trial (Boutelle 2011).

Of the two trials that did not report measure of variance, the mean change in BMI z score was reduced in both groups; -0.4 in the parent-only group and -0.1 in the parent-child group in the study by Golan 2006, and -0.16 in the parent-only group and -0.08 in the parent-child group in the study by Munsch 2008 . All trials had either high rates of non-completion across groups or differential non-completion rates between study groups and, therefore, these results need to be interpreted with caution. In the study by Collins 2011, the BMI was also reported, there were no significant differences in change from baseline between groups.

Five trials (six comparisons) reported BMI variables beyond the end of the intervention (Boutelle 2011, Janicke 2008, and Munsch 2008 after six months; Collins 2011 after 18 months; Golan 2006 after 12 months). In all five trials, this was the BMI $\mathrm{z}$ score. Two trials did not report SDs or information that could be used to estimate SDs and, therefore, these were not included in the pooled summary estimate (Golan 2006; Munsch 2008). Three trials (four comparisons) therefore provided data that could be analysed in a meta-analysis (Boutelle 2011; Collins 2011; Janicke 2008) (see Analysis 1.2). There was no substantial difference on BMI z score change between those in the parent-only interventions and those in the parent-child interventions ((MD -0.04 (95\% CI -0.15 to $0.08) ; \mathrm{P}=0.56 ; 267$ participants; 3 trials with 4 treatment groups; low quality evidence). There was moderate heterogeneity $\left(\mathrm{I}^{2}=\right.$ $37 \%)$; results were similar with a fixed-effect meta-analysis. There was a high risk of attrition bias in these three studies; we imputed SDs for two of these trials (Boutelle 2011; Collins 2011). In one of the two trials that did not report SDs, there was a reduction in
BMI $\mathrm{z}$ score in both intervention groups (-0.3 with parent-only versus -0.14 with parent-child) (Munsch 2008) . In the study by Golan 2006, there was a reduction in BMI z score in the parentonly group (-1.28) and a slight increase in BMI $\mathrm{z}$ score in the parent-child group (0.032).

Two trials also reported the change in percentage overweight at follow-up. In the study by Golan 2006, immediately after the intervention the overweight change in the parent-only group was $10 \%$ (SD 22) and in the parent-child group this was - $3 \%$ (SD 18) $(\mathrm{P}<0.05$ for between-group difference). At follow-up 12 months later, the change in percentage overweight was $-12 \%$ in the parentonly group and $0.4 \%$ in the parent-child group $(\mathrm{P}<0.05)$. In Munsch 2008, the change reported was a $-4 \%$ reduction at the end of treatment in the parent-only group and a $-2 . \%$ reduction at the end of treatment in the parent-child group (P not significant). At follow-up six months later, the reduction in per cent overweight was $-5 \%$ in the parent-only group and $-2 \%$ in the parent-child group (P not significant).

Collins 2011 reported body weight, adjusted for age, immediately after the intervention and at the longest period of follow-up (24 months). At six months (immediately after the intervention), in the parent-only group the change from baseline weight was 0.4 $\mathrm{kg}$ (SD 2); in the parent-child physical activity group this was $1.3 \mathrm{~kg}$ (SD 3.2) and in the parent-child physical activity plus diet group this was $0.1 \mathrm{~kg}$ (SD 2.9). At 24 months, in the parentonly intervention this was $-1.7 \mathrm{~kg}$ (SD 9.4), in the parent-child physical activity group this was $0.4 \mathrm{~kg}$ (SD 11) and in the parentchild physical activity plus diet group this was $-0.9 \mathrm{~kg}$ (SD 10.7). Three trials reported parental BMI (Boutelle 2011; Janicke 2008; Munsch 2008). The change from baseline parental BMI in the Janicke 2008 study was -0.9 (SD 1.6) in the parent-based group and -0.7 (SD 1.0) in the control group at the end of the intervention (five months) and was -0.6 (SD 2.4) in the parent-only group and 0.2 (SD 1.5) in the control group at the follow-up at 11 months. There were no substantial differences between groups at either time point $(\mathrm{P}=0.93$ at four months, $\mathrm{P}=0.17$ at 10 months). In the study by Munsch 2008, the change in parental BMI at the end of treatment (10 weeks) was 0.1 (SD not calculable) in the parent-only group and -0.04 (SD not calculable) in the parent-child group. At the end of follow-up (six months later), the change in parental BMI was - 0.1 (SD not calculable) in the parent-only group and 0.1 (SD not calculable) in the parent-child group. The study reported that there were no significant differences between groups. Boutelle 2011 reported BMI post-intervention (five months) and six months later. Parental BMI change was - 0.7 (SD 8.7) in the parent-only group and -0.1 (SD 7.4) in the parent-child group post intervention, and 0.1 (SD 9.1) in the parent-only group and 0.3 (SD 7.2) in the parent-child group at follow-up. Results were suggested by the authors to be likely to be non-inferior. There were high levels of non-completion in these trials. 


\section{Adverse events}

No trials reported adverse events.

\section{Secondary outcomes}

\section{Health-related quality of life and self esteem}

No trials reported health-related quality of life measures.

Immediately post the four-month intervention Janicke 2008 assessed the self esteem of the children on four subscales of the validated Self-Perception Profile for Children (SPPC) measure. These were social self esteem, athletic self esteem, physical self esteem and global self esteem. Results were reported for boys and girls separately because of gender differences in self esteem and, therefore, normative data were presented for the separate groups. An increase in score on this measure corresponds to increased self esteem. Changes from baseline were generally positive for all measures for both the parent-only and parent-child groups. There were no substantial differences between the groups seen at follow-up (analysis was for a treatment effect between the three interventions included in the study, no pairwise analysis was undertaken of the parent-only and parent-child groups).

\section{All-cause mortality}

No trials reported all-cause mortality.

\section{Morbidity}

No trials reported morbidity.

\section{Measures of body fat distribution}

One trial reported waist circumference adjusted for age and gender (waist z score) (Collins 2011). The change from baseline between groups at the immediate follow-up was: parent-only -0.3 (SD 0.3); parent-child physical activity -0.1 (SD 0.5); parent-child physical activity plus diet -0.2 (SD 0.5). At 24-month follow-up, change from baseline was: parent-only -3.9 (SD 9.9); parent-child physical activity -1.5 (SD 11.6); parent-child physical activity plus diet 1.1 (SD 11.1). Both sets of results should be interpreted in view of the differential and high rates of study non-completion.

\section{Behaviour change}

One trial used the Physical Activity Questionnaire for Older Children (Boutelle 2011). This was a seven-day recall measure designed to assess physical activity levels and consists of nine items, each being rated on a 5-point scale. At post-intervention follow-up and six months later, results for the parent-only group showed noninferiority to the parent-child group. Scores were 2.8 (SD 0.6) in the parent-only group and 2.7 (SD 1) in the parent-child group at immediate follow-up, and 4.2 (SD 3.7) in the parent-only group versus 2.8 (SD 0.7) in the parent-child group at follow-up six months later. Non-completion rates were high in both groups in this study.

Munsch 2008 reported outcomes on the German version of the Child Behaviour CheckList (CBCL), reporting the global score and the subscales of CBCL externalising, CBCL internalising and CBCL social problems at the end of treatment (10 weeks) and six months later. There were no substantial differences between the parent-only group and parent-child groups on any of these scales (data provided by study author).

The study by Collins 2011 used an objective measure of physical activity from the Actigraph 7164 uniaxial accelerometer to assess counts per minute over an eight-day period. The total physical activity recorded increased in all groups at the immediate point of assessment (six months) but there were no substantial differences between groups. At the longest point of follow-up (12 months), the physical activity recorded increased in the two parent-child groups and decreased in the parent-only group, but there were no substantial differences seen. In addition, this study measured parental report of screen behaviours by a validated measure, the Children's Leisure Activities Study Survey. The total screen time use decreased in all three groups at both measurements, but there were no substantial differences between groups.

\section{Participants' views of the intervention}

The study by Janicke 2008 asked parents whether they would be prepared to join the programme again. In the parent-only group, $88 \%$ of parents responded that they would and $12 \%$ responded that they may be prepared to join the programme again. In the parent-child intervention, $91 \%$ of parents responded that they would and $9 \%$ responded that they may be prepared to join the programme again. Children in the parent-child group were asked if it was true that "Overall, this was a good program", where $85 \%$ responded 'really true'; $12 \%$ responded 'sort of true' and $3 \%$ responded 'sort of not true'.

\section{Parent-child relationship or assessment of parenting}

No trials reported outcomes assessing parent-child relationships or an assessment of parenting.

\section{Socioeconomic effects}

No trials reported outcomes assessing socioeconomic effects.

\section{Parent-only interventions versus waiting list controls}

Six trials reported eight comparisons of a parent-only intervention and a waiting list control (Aragona 1975; Golley 2007; Janicke 
2008; Jansen 2011; Moens 2012; West 2010). To allow consideration of the effects of the interventions, we considered outcomes from the longest period of follow-up and any post intervention follow-up. The period for the post intervention follow-up in these trials ranged from three to six months and the period for the longest point of follow-up ranged from six to 12 months. Two trials did not report a period of follow-up beyond the post intervention follow-up (Moens 2012; West 2010). Losses to follow-up ranged from $6 \%$ to $40 \%$ at the post intervention follow-up and $18 \%$ to $60 \%$ at the longest period of follow-up (see Table 1). In the trials by Janicke 2008, Jansen 2011, West 2010, and Aragona 1975, there was a differential rate of losses to follow-up between groups. In the trials by Golley 2007 and Aragona 1975, losses to followup at both time points were high: between $14 \%$ and $24 \%$ in the study by Golley 2007 and up to 69\% in the study by Aragona 1975. Aragona 1975 had a very small sample size of five participants per treatment group. These factors need to be considered when interpreting the results of the trials.

\section{Primary outcomes}

\section{Changes in body mass index and body weight}

Three trials reported BMI variables at follow-up post intervention (Janicke 2008 at four months; West 2010 at 12 weeks; Jansen 2011 at three months). In two trials, this was the BMI z score (Janicke 2008; West 2010), and in the third trial, this was the BMI percentile (Jansen 2011). A fourth study reported individual participant data for weight and height and we calculated the mean BMI from these data (Aragona 1975). Another study reported adjusted BMI based on parental report of weight and height and as the data were, therefore, not reliable, we have not discussed them further here (Moens 2012).

The meta-analysis for the change in the BMI z score comparing the parent-only group and the waiting list control group showed an $\mathrm{MD}$ of -0.12 ((95\% CI -0.21 to -0.04$)$; $\mathrm{P}=0.003 ; 153$ participants; 2 trials; low quality evidence; Analysis 2.1). Janicke 2008 had a high risk of attrition and reporting bias, and West 2010 had a high risk of selection and performance bias. We imputed SDs for West 2010.

At the longest point of follow-up, four trials (six comparisons) reported BMI variables (Golley 200712 months post baseline; Janicke 2008 and Jansen 2011 six months after; Aragona 197539 weeks after). In two trials, this was the BMI z score (Golley 2007; Janicke 2008), in one trial, this was the BMI percentile (Jansen 2011), and in one trial this was the BMI (Aragona 1975).

Meta-analysis for the change in BMI z score comparing the parentonly group with the waiting list control group showed a MD of 0.10 ((95\% CI -0.19 to -0.01$) ; \mathrm{P}=0.04 ; 136$ participants; 2 trials with 3 treatment arms; low quality evidence; Analysis 2.2). Janicke
2008 had a high risk of attrition and reporting bias, Golley 2007 had a high risk of reporting bias.

Jansen 2011 found no significant difference between the parentonly intervention and the waiting list control in BMI percentile change from baseline (MD - 1.90 (95\% CI -3.76 to -0.04); Analysis 2.4). Aragona 1975 found no significant difference between either the parent-only plus reinforcement group or the parent-only group and the waiting list control on change from baseline BMI (Analysis 2.6). These data were based on small numbers and the rate of noncompletion was high and these data have not been combined in a meta-analysis.

In Jansen 2011, there was a treatment effect in favour of the parentonly intervention for BMI percentile change $(-0.48$ (95\% CI $0.89,0.07$ ); Analysis 2.3); however, Jansen 2011 was at a high risk of bias owing to nine families originally allocated to the waiting list control group being included in the data set for the parent-only intervention, and, therefore, these data should be considered with caution. In Aragona 1975, there was a reduction from baseline BMI in both the parent-only plus reinforcement group and the parent-only intervention group compared with the waiting list control group (Analysis 2.5). However, we did not perform a metaanalysis because these data were based on small numbers and the rate of non-completers was high.

We undertook no sensitivity analyses on BMI change for trials with high loss to follow-up as all of the trials had high loss to follow-up. Therefore, caution is recommended in the interpretation of these data.

Aragona 1975 found a reduction in weight (which we converted to kilograms) in all three intervention groups at the end of the intervention period (parent-only with reinforcement $-5.1 \mathrm{~kg}$ (SD 0.8 ); parent-only without reinforcement $-4.3 \mathrm{~kg}$ (SD 2.9); control $-0.2 \mathrm{~kg}(\mathrm{SD} 2.1))$. At the longest point of follow-up, there was a decrease in weight in the parent-only with reinforcement group (0.3 (SD 2.4)) and increases in weight in the parent-only without reinforcement group (3.3 (SD 2.9) and the control group (4.7 (SD 3.4)).

Two trials reported the change in parental BMI (Janicke 2008; Jansen 2011). In the study by Janicke 2008, there was a decrease in parental BMI in both the parent-only group and the waiting list control group at the post intervention follow-up (parent-only -0.9 (SD 1.6) versus waiting list control -0.7 (SD 2.6); $\mathrm{P}=0.93$ based on a three-way comparison). At the longest period of followup (at 10 months), there was also a decrease in parental BMI from baseline, albeit smaller (parent-only -0.6 (SD 2.4) versus waiting list control -0.6 (SD 3.6); $\mathrm{P}=0.17$, based on a threeway comparison). In the study by Jansen 2011, the parental BMI decreased in the parent-only group and increased in the waiting list control group immediately post intervention (parent-only 0.3 (SD 4.5) versus waiting list control 0.1 (SD 6.3)), and at the longest period of follow-up six months post intervention (parentonly -0.2 (SD 4.5) versus waiting list control 0.1 (SD 6.3)). 


\section{Adverse events}

No trials reported adverse events.

\section{Secondary outcomes}

\section{Health-related quality of life and self esteem}

No trials reported health-related quality of life.

The study by Jansen 2011 assessed self esteem on the SPPC. This validated measure comprises of six subscales, two of which were of interest and reported by the study authors (physical appearance, global self worth). Scores for each item range from 1 to 4 with higher scores relating to poorer outcome. At the post treatment assessment (three months), the physical appearance rating from the parent-only intervention group's children was 15.9 (SD 5) and in the waiting list control this was 16.1 (SD 4.7). Six months later, the ratings were 16 (SD 5.3) for the parent-only group and 15.7 (SD 4.7) for the waiting list control group. There was no substantial main effect between groups (comparing both groups over both time periods). On the global self worth scale, the parentonly intervention rating was 18.8 (SD 4.2) and the waiting list control rating was 20.3 (SD 4.2) at the three month post intervention follow-up. At the final follow-up, these ratings were 19 (SD 4.9) for the parent-only group and 20.2 (SD 4.1) for the waiting list control group. There was no substantial main effect between groups (comparing both groups over both time periods).

Janicke 2008 assessed the self esteem of the children on four subscales (social self esteem, athletic self esteem, physical self esteem and global self esteem) of the SPPC measure at the end of the four-month intervention. Results were reported for boys and girls separately. Changes from baseline were generally positive for all measures for both the parent-only and waiting list control groups. There were no substantial differences between the groups at follow-up.

\section{All-cause mortality}

No trials reported all-cause mortality.

\section{Morbidity}

No trials reported morbidity.

\section{Measures of body fat distribution}

Golley 2007 calculated the waist circumference z score. At the final follow-up (at 12 months), the mean change in score was - 0.3 (SD $0.5)$ in the parent-only intense group, $-0.2(0.5)$ in the parentonly group and $-0.02(0.6)$ in the waiting list control group. These reductions in waist circumference $\mathrm{z}$ scores were not substantially different between groups.

\section{Behaviour change}

West 2010 reported the Lifestyle Behaviour Checklist (LBC), which is a measure of child weight-related problem behaviour and includes items on eating behaviours and physical activity, yielding scores on two scales, the LBC problem scale (lower scores indicate better outcome) and the LBC confidence scale (higher scores indicate better outcome). At the post intervention follow-up at 12 weeks, the LBC problem scores were 59.4 (SD 20.7) in the parentonly group and 73.8 (SD 19.3) in the waiting list control group. The LBC confidence scores were 204.4 (SD 37.5) in the parentonly group compared with 165.8 (SD 46.4) in the waiting list control group $(\mathrm{P}<0.0125$ on both scales in favour of the parentonly intervention).

\section{Participants' views of the intervention}

For the participants' views of the parent-only interventions in the study by Golley 2007 see 'Parent-only interventions versus parentonly interventions' below.

\section{Parent-child relationship or assessment of parenting}

The study by West 2010 reported data from the Parenting Scale, which is a validated measure of parental discipline practices. It has 30 items and parents indicate their tendencies to use specific discipline strategies using 7-point Likert scales, where 7 indicates a high probability of making the discipline mistake and 1 indicates a high probability of using an effective, alternative discipline strategy. At the post intervention follow-up at 12 weeks the Parenting Scale scores were 2.7 (SD 0.7) in the parent-only group and 3.4 (SD 0.5) in the waiting list control group $(\mathrm{P}<0.0125$ in favour of the parent-only intervention; low quality evidence).

\section{Socioeconomic effects}

No trials reported socioeconomic effects.

\section{Parent-only interventions versus minimal contact interventions}

Seven trials reported 10 comparisons of a parent-only intervention and a minimal contact control (Esfarjani 2013; Estabrooks 2009; Mazzeo 2014; Resnick 2009; Small 2013; Resnicow 2015; van Grieken 2013). To allow consideration of the effects of the interventions, we considered outcomes from the longest period of follow-up and any post intervention follow-up. The period for the post intervention follow-up in these trials ranged from three to six months in all but two trials (12 months for van Grieken 2013; 24 months for Resnicow 2015), and the period for the longest point of follow-up ranged from six to 12 months (one study, Esfarjani 2013, did not state the duration of follow-up). One study had a planned six-month follow-up but did not report results as the loss 
to follow-up was high (Mazzeo 2014, 68\% to 73\% loss to followup). Two trials did not report a period of follow-up beyond the end of the intervention (Resnick 2009; Resnicow 2015). Losses to follow-up in the included trials ranged from $0 \%$ to $34 \%$ at the post intervention follow-up and were between $17 \%$ and $38 \%$ at the longest period of follow-up (see Table 1). These need to be considered when interpreting the results of the trials below.

\section{Primary outcomes}

\section{Changes in body mass index and body weight}

Six trials (eight comparisons) reported BMI variables at follow-up post intervention. In one study (two comparisons), this was the BMI z score (Estabrooks 2009), and in the remaining trials, this was the BMI percentile (Mazzeo 2014; Resnick 2009; Resnicow 2015; Small 2013) or BMI (Esfarjani 2013). The period for the outcome assessment differed between trials (Esfarjani 2013 at six months; Estabrooks 2009 at 12 to 24 weeks; Mazzeo 2014 at 12 weeks; Resnick 2009 at 18 to 30 weeks; Resnicow 2015 at 24 months; Small 2013 at 17 weeks).

Meta-analysis for change in BMI z score between the parent-only groups and the control group showed an MD of -0.00 ((95\% CI 0.08 to 0.08$) ; \mathrm{P}=0.99 ; 170$ participants; 1 trial with 3 treatment arms; low quality evidence). There was a high risk of attrition bias and reporting bias for Estabrooks 2009 and we imputed SDs.

Four trials reported the change in BMI percentile; however, because of lack of standardisation these could not be pooled. None of the studies demonstrated treatment effects (Analysis 3.3).

For BMI change from the trial by Esfarjani 2013, there was no difference between comparison groups (Analysis 3.5).

At the longest point of follow-up, four trials (five comparisons) reported BMI variables. In one trial (two comparisons, Estabrooks 2009), this was the BMI $z$ score, in one trial this was the BMI percentile (Small 2013), and in the remaining two trials this was the BMI (Esfarjani 2013; van Grieken 2013). The duration of follow-up varied, in the study by Estabrooks 2009 and Small 2013, this was six months after the end of the intervention; the study by Esfarjani 2013 did not report the duration of follow-up after the six month intervention; in the study by van Grieken 2013, follow-up was at 24 months following an intervention of up to 12 months.

Meta-analysis for the change in BMI z score comparing the parentonly group with the minimal contact control group showed a MD of 0.01 ( (95\% CI -0.07 to 0.09$)$; $\mathrm{P}=0.81 ; 165$ participants; 1 trial with 3 treatment arms; low quality evidence; Analysis 3.2).

The one study reporting BMI percentile found no a substantial treatment effect on BMI percentile change (MD - 0.93 (95\% CI 3.49 to 1.63 ); Analysis 3.4).

Meta-analysis for change from baseline BMI showed an MD of 0.12 ((95\% CI -0.39 to 0.15$)$; $\mathrm{P}=0.39$; 614 participants; 2 trials; low quality evidence; Analysis 3.6) (Esfarjani 2013; van Grieken 2013). In the cluster trial, the study authors calculated an intracluster correlation coefficient of 0.06 for BMI and the numbers analysed appear to have been adjusted appropriately (van Grieken 2013). All of these trials had high loss to follow-up, which should be considered when interpreting these results.

No trials reported the parental BMI.

In the study by Esfarjani 2013, weight in kilograms was also reported as an outcome. The change from baseline weight at the end of the six-month intervention was $1.8 \mathrm{~kg}$ (SD 4.9) in the parent-only group and $2.6 \mathrm{~kg}$ (SD 5) in the control group, giving a difference of $0.8 \mathrm{~kg}(95 \% \mathrm{CI}-2.67$ to 1.1$)$. At the longest point of follow-up (not defined), the weight change for the two groups were $5 \mathrm{~kg}$ (SD 5.1) for the parent-only group and $5.7 \mathrm{~kg}$ (SD 5.17) for the control group (difference $0.7 \mathrm{~kg}$ (95\% CI -2.6 to 1.2 ).

\section{Adverse events}

No trials reported adverse events.

\section{Secondary outcomes}

\section{Health-related quality of life and self esteem}

One study reported narratively that there were no improvements in health-related quality of life but reported no data (Mazzeo 2014). No trials reported self esteem.

\section{All-cause mortality}

No trials reported all-cause mortality.

\section{Morbidity}

No trials reported morbidity.

\section{Measures of body fat distribution}

Small 2013 reported waist and waist-to-height ratio. At the post intervention assessment (three months), waist circumference was 28.2 inches (SD 3.8) in the parent-only intervention compared with 28.4 inches (SD 3.7) in the control group (1 inch $=2.5 \mathrm{~cm}$ ). At the final assessment (six months later), the waist circumference in the parent-only group was 29.5 inches (SD 3.5) compared with 28.9 inches (3.7) in the control group. The waist-to-height ratio at three months was 0.58 (SD 0.08) in the parent-only group and 0.60 (SD 0.06) in the control group. The corresponding values at six-month follow-up were 0.59 (SD 0.09) in the parent-only group and 0.59 (SD 0.08) in the control group. The trial reported that these results were not statistically significant between groups. The study by Esfarjani 2013 reported waist circumference. The change from baseline waist circumference at the end of the six- 
month intervention was $-1.0 \mathrm{~cm}$ (SD 5.8) in the parent-only group and $1.5 \mathrm{~cm}$ (SD 5.1) in the control group, with a difference of 2.5 $\mathrm{cm}(95 \% \mathrm{CI}-4.6$ to -0.4$)$. At the longest point of follow-up (not defined), the changes in waist circumference were $2 \mathrm{~cm}$ (SD 5.8) in the parent-only group and $3.5 \mathrm{~cm}$ (SD 5.28) in the control group, with a difference of $1.5 \mathrm{~cm}$ (95\% CI -3.6 to 0.6). van Grieken 2013 also reported waist circumference at 24-month follow-up. In the parent-only group the change in waist circumference was $7.2 \mathrm{~cm}$ (SD 5.5) compared with $7.3 \mathrm{~cm}$ (SD 5.3) in the control group, with a difference of $0.1 \mathrm{~cm}(95 \% \mathrm{CI}-1.1$ to 0.8$)$.

Esfarjani 2013 reported hip circumference. There was a change in hip circumference in the parent-only group at the end of the intervention of $-0.5 \mathrm{~cm}$ (SD 4.9) compared with $1.4 \mathrm{~cm}$ (SD 4.9) in the control group, with a difference of $1.9 \mathrm{~cm}(95 \% \mathrm{CI}-3.8$ to 0.1 ). At the longest point of follow-up (not defined), the change in hip circumference was $2.1 \mathrm{~cm}$ (SD 4.8) in the parent-only group and $3.7 \mathrm{~cm}(\mathrm{SD} 5.1)$ in the control group, with a difference of 1.6 $\mathrm{cm}(95 \% \mathrm{CI}-3.5$ to 0.2$)$.

\section{Behaviour change}

No trials reported validated measures assessing behaviour change.

\section{Participants' views of the intervention}

The study by Mazzeo 2014 sought parental views at completion of the parent-only intervention. Parents either strongly agreed (79\%) or moderately agreed (21\%) with the statement "I enjoyed attending each NOURISH session.” In addition, 92\% strongly agreed that they would recommend the intervention to other parents, 91\% strongly or moderately agreed that the sessions had helped them eat in a healthier manner and $78 \%$ said they were exercising more. The study also reported examples of qualitative responses received from parents in interviews, both positive and negative (e.g. "I really enjoyed them and hearing what other parents concerns were like mine" and "I was excited at first but once it started, it was sometimes difficult to get there, park, and stay focused for 90 min after working all day").

Resnick 2009 asked parents a questions about the materials used and both groups appeared to be satisfied with the materials. Of 20 parents in the parent-only group, 13 (65\%) reported reading all the study materials, the corresponding rate in the control group was $17 / 22(77 \%)$. Some 17 (94\%) parents in the parent-only group and $18(82 \%)$ of parents in the control group stated that they would recommend the programme to other families. The parents in the intervention group were also asked if they found the community health workers to be helpful and 16/20 (80\%) stated "yes". The trial authors reported responses to other questions.

\section{Parent-child relationship or assessment of parenting}

Mazzeo 2014 reported parental concern about the child's weight. This was one of seven subscales of the Child Feeding Questionnaire (results of other subscales were not reported). Parental concern decreased from 4.7 (SD 0.5) to 4.6 (SD 0.7) in the parent-only group and from 4.7 (SD 0.5) to 4.7 (SD 0.5) in the control group at the follow-up immediately following the 12-week intervention ( $\mathrm{P}$ $=0.041$ in favour of the parent-only intervention; 93 participants; 1 trial; low quality evidence).

\section{Socioeconomic effects}

No trials reported socioeconomic effects.

\section{Parent-only interventions versus parent-only interventions}

Seven trials reported nine comparisons of two different parentonly interventions (Aragona 1975; Estabrooks 2009; Golley 2007; Magarey 2011; Raynor 2012a; Raynor 2012b; Resnicow 2015). The point of post intervention follow-up was three months in one study (Aragona 1975); six months in five studies (Estabrooks 2009; Golley 2007; Magarey 2011; Raynor 2012a; Raynor 2012b); and 24 months in one study (Resnicow 2015). The longest points of follow-up ranged from 12 to 24 months (all trials except Magarey 2011 had a 12-month follow-up, Resnicow 2015 only had one period of follow-up at the end of the intervention). Losses to followup in the included trials ranged from $3 \%$ to $40 \%$ at the post intervention follow-up and were between $8 \%$ and $60 \%$ at the longest period of follow-up (see Table 1). These need to be considered when interpreting the results of the trials.

\section{Primary outcomes}

\section{Changes in body mass index and body weight}

Five trials reported BMI z score and Analysis 4.1 and Analysis 4.2 show the change from baseline for each study. No meta-analysis was possible because there was little or no consistency between trial interventions and comparators. A narrative synthesis of the results of the trials follows.

The study by Estabrooks 2009 (a three-arm trial, see above for other comparisons) compared two parent-only interventions. Parents either attended a group intervention that addressed behavioural health skills and knowledge or a group intervention that addressed the same issues but also included 10 automated telephone counselling sessions over a period of six months. Both groups also utilised a workbook aimed at promoting healthy habits and physical activity throughout the interventions. Change in BMI $z$ score at the immediate follow-up at six months showed an MD of -0.04 ((95\% CI -0.16 to 0.08$) ; \mathrm{P}=0.51)$ and at the followup 12 months later an MD of -0.06 ((95\% CI -0.18 to 0.06$)$; $\mathrm{P}=$ $0.34)$. Losses to follow-up were above $20 \%$ in both groups at both time points. 
In a three-arm study, Golley 2007 had two parent-only interventions: parents either attended a behavioural change parenting skills training intervention or the same intervention with seven additional intensive lifestyle support group sessions. At six months, BMI $z$ score change showed a MD of -0.09 ((95\% CI -0.38 to $0.20) ; \mathrm{P}=0.54)$ where there was a $24 \%$ drop-out rate in both groups at this time point. Similarly, at 12 months' follow-up, the MD was -0.09 ( $(95 \% \mathrm{CI}-0.32$ to 0.14$) ; \mathrm{P}=0.44)$. The non-completion rate at this point of follow-up was $18 \%$ in the intensive group and $22 \%$ in the standard group intervention.

Magarey 2011 compared two parent-only interventions, a healthy lifestyle group (which included recommendations, practical skills and monitoring aspects) and a group that had the same healthy lifestyle intervention with the addition of four parenting skills sessions. BMI $\mathrm{z}$ score change at the completion of the intervention at six months showed an MD of -0.07 ((95\% CI -0.29 to 0.15$)$; $\mathrm{P}=0.54)$ and at the 24-month follow-up, an MD of $0.03((95 \%$ $\mathrm{CI}-0.24$ to 0.30 ); $\mathrm{P}=0.82$ ). Non-completion rates in this study were between $17 \%$ and $22 \%$ at the post intervention follow-up and $36 \%$ and $39 \%$ at the 24 -month follow-up.

One publication reported two trials and each trial had three arms, a standard arm that was a growth monitoring intervention and two additional parent-only interventions. In the first trial, the two additional interventions focused on diet, with one intervention focusing on decreasing sugar and salty snack food and drink consumption and the second intervention focusing on increasing fruit, vegetable and low-fat dairy intake (Raynor 2012a). In the second trial, the two additional interventions focused on diet and physical activity, with one intervention focusing on decreasing sugar intake and increasing physical activity and the second intervention focusing on increasing low-fat intake and decreasing sedentary lifestyles such as watching television (Raynor 2012b). The completion rates in these trials were better than the other trials, with between $3 \%$ and $12 \%$ of participants not completing the study. At the point of completion of the intervention (six months) in the Raynor 2012a trial, the difference in BMI z score change between the growth monitoring group and the group focusing on decreasing sugar consumption was -0.04 ( $(95 \%$ CI -0.17 to $0.09) ; \mathrm{P}=0.53$; data provided by study authors). The difference between the growth monitoring group and the group focusing on increasing healthy food consumption was -0.01 ((95\% CI -0.14 to 0.12$) ; \mathrm{P}=0.88$; data provided by study authors). Similar results were also seen at 12 months in the decreasing sugar group with an MD of -0.04 ((95\% CI -0.19 to 0.11$)$; $\mathrm{P}=0.61)$, and in the increasing healthy food group with an MD of -0.02 ((95\% CI 0.17 to 0.13 ); $\mathrm{P}=0.80$ ) (data provided by study authors). At the point of completion of the Raynor 2012b trial, BMI z score change between the group focusing on increasing activity compared with the growth monitoring group was -0.69 ( $(95 \% \mathrm{CI}-0.83$ to -0.55$)$; $\mathrm{P}<0.00001$; data provided by study authors). The difference between the group focusing on reducing sedentary behaviours compared with the growth monitoring group was -0.70 (( $95 \% \mathrm{CI}$ -
0.86 to -0.54$) ; \mathrm{P}<0.00001$; data provided by study authors). At 12 months, there was a different pattern, with neither comparisons showing substantial differences between groups: increasing activity group MD 0.01 ( (95\% CI -0.17 to 0.19$) ; \mathrm{P}=0.92)$ and decreasing sedentary behaviours group MD -0.03 ((95\% CI -0.24 to 0.18$) ; \mathrm{P}=0.78$ ) (data provided by study authors).

Aragona 1975 reported the weight and height of individual participants and mean BMI was calculated from these data. Both parent-only interventions included training in nutrition, exercise and behaviour, and parents contracted to the investigators regarding a weight loss goal for their child. One intervention also taught reinforcement techniques. Immediately after the intervention, and at the longest point of follow-up, there were no substantial differences in change from baseline BMI seen (Analysis 4.3; Analysis 4.4).

The study by Resnicow 2015 reported the BMI percentile at the end of the 24-month intervention. There were no differences between the two parent-only interventions (Analysis 4.5).

\section{Adverse events}

The trials by Raynor 2012a; Raynor 2012b reported narratively that there were no serious adverse events.

\section{Secondary outcomes}

\section{Health-related quality of life and self esteem}

No trials reported health-related quality of life or self esteem.

\section{All-cause mortality}

No trials reported all-cause mortality.

\section{Morbidity}

No trials reported morbidity.

\section{Measures of body fat distribution}

Golley 2007 calculated waist circumference z score. At the post intervention follow-up (six months), the mean change in waist $\mathrm{z}$ score was -0.27 (SD 0.7) in the parent-only intense group and 0.12 (SD 0.61) in the parent-only group. These reductions in waist circumference $\mathrm{z}$ scores were not significantly different between groups. At the final follow-up (at 12 months), the mean change in score was - 0.31 (SD 0.53) in the parent-only intense group and $-0.17(0.50)$ in the parent-only group. These reductions in waist circumference $\mathrm{z}$ scores were not statistically significantly different between groups. 


\section{Behaviour change}

No trials reported behaviour changes.

\section{Participants' views of the intervention}

Golley 2007 reported a programme evaluation. Response rates varied, with $26 / 31$ in the parenting and intensive lifestyle group responding and 10/29 in the parenting group responding. All participants in both groups rated the quality of the service provided during the interventions as good to excellent. Fifty per cent (13/ 26) of participants in the parenting and intensive lifestyle training group and $80 \%(8 / 10)$ in the parent-only group said they either generally or definitely received the type of help they required from the respective programme. One hundred per cent of parents in the parenting group and $85 \%(22 / 26)$ in the parenting and intensive lifestyle group stated they were satisfied to very satisfied with the amount of help received. One hundred per cent of parents in the parenting group and $92 \%$ (24/26) in the parenting and intensive lifestyle group stated they were helped somewhat or helped a great deal by the intervention. The study authors provided other responses.

\section{Parent-child relationship or assessment of parenting}

One study reported results from the Alabama Parenting Questionnaire (satisfaction, efficacy, involvement, positive parenting, poor monitoring, inconsistent discipline, corporal punishment), which is a validated measure (Magarey 2011). However, outcomes were reported only for the total group, and no comparisons between the two treatment groups were provided.

\section{Socioeconomic effects}

No trials reported socioeconomic effects.

\section{Subgroup analyses}

We did not perform subgroups analyses because there were not enough trials to estimate effects in various subgroups.

\section{Sensitivity analyses}

We did not perform any sensitivity analyses because there were not enough trials included in the analyses.

\section{Assessment of reporting bias}

We did not draw funnel plots due to limited number of trials with data included in any one analysis.

\section{Ongoing trials}

We found 10 ongoing RCTs (see Characteristics of ongoing studies table). Nine of these RCTs are parallel trials, one is a cluster trial. The ages of the participants in these trials incorporate the range of 2 to 6 years in six trials, 7 to 13 years in two trials and were not reported in three trials. In five trials, the target population are children classed as overweight (various definitions), in two trials the target population are children classed as obese, and in four trials the population is described as overweight or obese. Five trials are comparing a parent-only intervention with another parent-only intervention; three trials are comparing a parent-only intervention with a parent-child intervention; three trials are comparing a parent-only intervention with a minimal contact intervention and one trial is comparing a parent-only intervention to no treatment. The primary outcome is the BMI $\mathrm{z}$ score in nine of the 10 ongoing trials; in the one other trial, this is weight change. The estimated study completion dates, where reported, range from January 2014 to November 2016. 


\begin{tabular}{|c|c|c|c|c|c|c|c|}
\hline 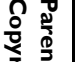 & A D D I T IONA & S U M M A R Y & F F INDINGS & [Explanation] & & & \\
\hline$\frac{7}{0}$ & Parent-only interventions & vs. waiting list control for & childhood overweight or c & obesity & & & \\
\hline 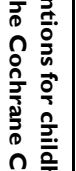 & $\begin{array}{l}\text { Population: children with } \\
\text { Settings: outpatients; com } \\
\text { Intervention: parent-only } \\
\text { Comparison: waiting list c }\end{array}$ & $\begin{array}{l}\text { overweight or obesity } \\
\text { nmunity } \\
\text { interventions } \\
\text { control }\end{array}$ & & & & & \\
\hline 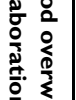 & Outcomes & Waiting list & Parent-only & $\begin{array}{l}\text { Relative effect } \\
(95 \% \mathrm{CI})\end{array}$ & $\begin{array}{l}\text { No of participants } \\
\text { (trials) }\end{array}$ & $\begin{array}{l}\text { Quality of the evidence } \\
\text { (GRADE) }\end{array}$ & Comments \\
\hline 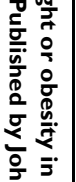 & $\begin{array}{l}\text { BMI z score change ( } x \text { * } \\
\text { SD) } \\
\text { Follow-up: } 40-48 \text { weeks }\end{array}$ & $\begin{array}{l}\text { The mean BMI } z \text { score } \\
\text { change ranged across } \\
\text { control groups from }-0 \text {. } \\
13 \text { to } 0.02\end{array}$ & $\begin{array}{l}\text { The mean BMI } z \text { score } \\
\text { change in the intervention } \\
\text { groups was } 0.1 \text { lower ( } 0 \text {. } \\
19 \text { lower to } 0.01 \text { lower) }\end{array}$ & - & $136(2)$ & $\begin{array}{l}\oplus \oplus \bigcirc \bigcirc \\
\mathbf{l o w}^{a}\end{array}$ & $\begin{array}{l}\text { Lower scores indicate im- } \\
\text { proved weight loss }\end{array}$ \\
\hline 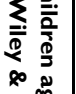 & Adverse events & See comment & See comment & See comment & See comment & See comment & $\begin{array}{l}\text { No trials reported adverse } \\
\text { events }\end{array}$ \\
\hline 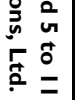 & $\begin{array}{l}\text { Health-related quality of } \\
\text { life }\end{array}$ & See comment & See comment & See comment & See comment & See comment & $\begin{array}{l}\text { No trials reported health- } \\
\text { related quality of life }\end{array}$ \\
\hline רై & All-cause mortality & See comment & See comment & See comment & See comment & See comment & $\begin{array}{l}\text { No trials reported all- } \\
\text { cause mortality }\end{array}$ \\
\hline & Morbidity & See comment & See comment & See comment & See comment & See comment & $\begin{array}{l}\text { No trials reported morbid- } \\
\text { ity }\end{array}$ \\
\hline N & $\begin{array}{l}\text { Parent-child relation- } \\
\text { ship or assessment of } \\
\text { parenting } \\
\text { (parenting scale (PS), } 30 \\
\text { items, scored from } 1 \text { to } \\
7 \text {; lower scores indicate } \\
\text { more effective parental } \\
\text { discipline practices) } \\
\text { Follow-up: } 12 \text { weeks }\end{array}$ & $\begin{array}{l}\text { The mean PS score for } \\
\text { the control group was } 3 \text {. } \\
4\end{array}$ & $\begin{array}{l}\text { The mean PS score in the } \\
\text { intervention group was } 0 \text {. } \\
6 \text { points lower }\end{array}$ & - & $101(1)$ & $\begin{array}{l}\oplus \oplus \bigcirc \bigcirc \\
\mathbf{l}_{\mathbf{0}} \mathbf{w}^{a}\end{array}$ & - \\
\hline
\end{tabular}




$\begin{array}{lllll}\text { Socioeconomic effects } & \text { See comment } & \text { See comment } & \text { See comment } & \text { See comment }\end{array}$

*The basis for the assumed risk (e.g. the median control group risk across trials) is provided in footnotes. The corresponding risk (and its $95 \%$ confidence interval) is based on the assumed risk in the comparison group and the relative effect of the intervention (and its $95 \% \mathrm{Cl}$ ).

BMI: body mass index; CI: confidence interval; PS: parenting scale; SD: standard deviation.

GRADE Working Group grades of evidence

High quality: Further research is very unlikely to change our confidence in the estimate of effect.

Moderate quality: Further research is likely to have an important impact on our confidence in the estimate of effect and may change the estimate.

Low quality: Further research is very likely to have an important impact on our confidence in the estimate of effect and is likely to change the estimate. Very low quality: We are very uncertain about the estimate.

" A BMI z score or standard deviation score indicates how many units (of the standard deviation) a child's BMI is above or below the average BMI value for their age group and sex. For instance, a $z$ score of 1.5 indicates that a child' is 1.5 standard deviations above

the average value, and a z score of -1.5 indicates a child is 1.5 standard deviations below the average value" (Noo NHS 2011).

${ }^{a}$ Downgraded by one level because of serious risk of attrition bias and one level for serious imprecision (see Appendix 9). 


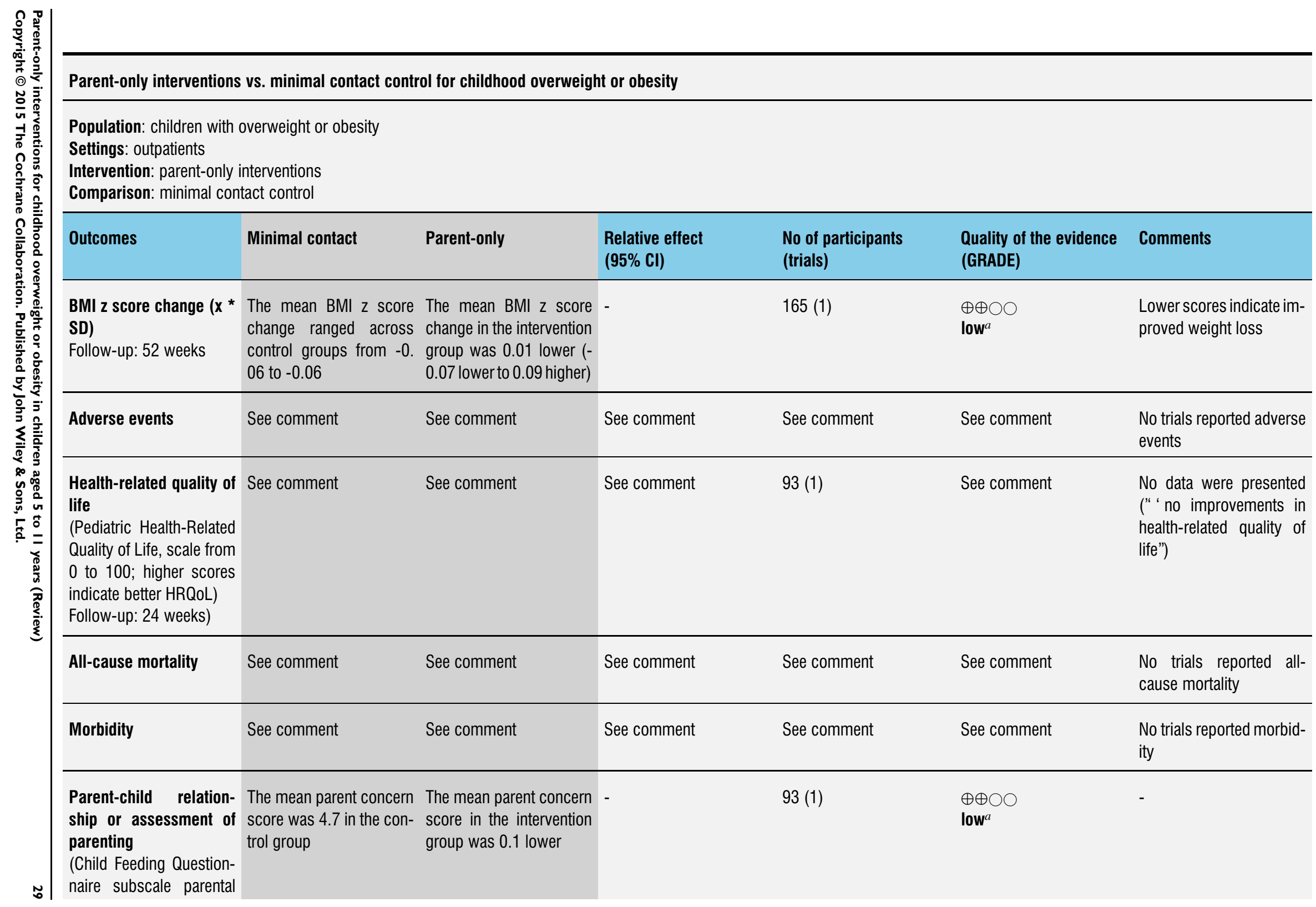


concern (total of 7 sub-

scales), score range $3-$

15 ; higher scores indicate

greater parental concern)

Follow-up: 12 weeks

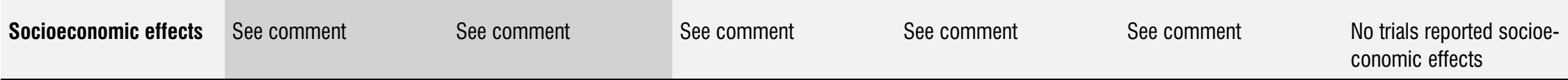

*The basis for the assumed risk (e.g. the median control group risk across trials) is provided in footnotes. The corresponding risk (and its $95 \%$ confidence interval) is based on the assumed risk in the comparison group and the relative effect of the intervention (and its $95 \% \mathrm{Cl}$ ).

BMI: body mass index; Cl: confidence interval; HRQoL: health-related quality of life; SD: standard deviation.

GRADE Working Group grades of evidence

High quality: Further research is very unlikely to change our confidence in the estimate of effect.

Moderate quality: Further research is likely to have an important impact on our confidence in the estimate of effect and may change the estimate.

Low quality: Further research is very likely to have an important impact on our confidence in the estimate of effect and is likely to change the estimate.

Very low quality: We are very uncertain about the estimate.

' A BMI $z$ score or standard deviation score indicates how many units (of the standard deviation) a child's BMI is above or below the average BMI value for their age group and sex. For instance, a z score of 1.5 indicates that a child' is 1.5 standard deviations above the average value, and a $z$ score of -1.5 indicates a child is 1.5 standard deviations below the average value" (Noo NHS 2011).

${ }^{a}$ Downgraded by one level because of serious risk of attrition bias and one level for serious imprecision (see Appendix 9). 


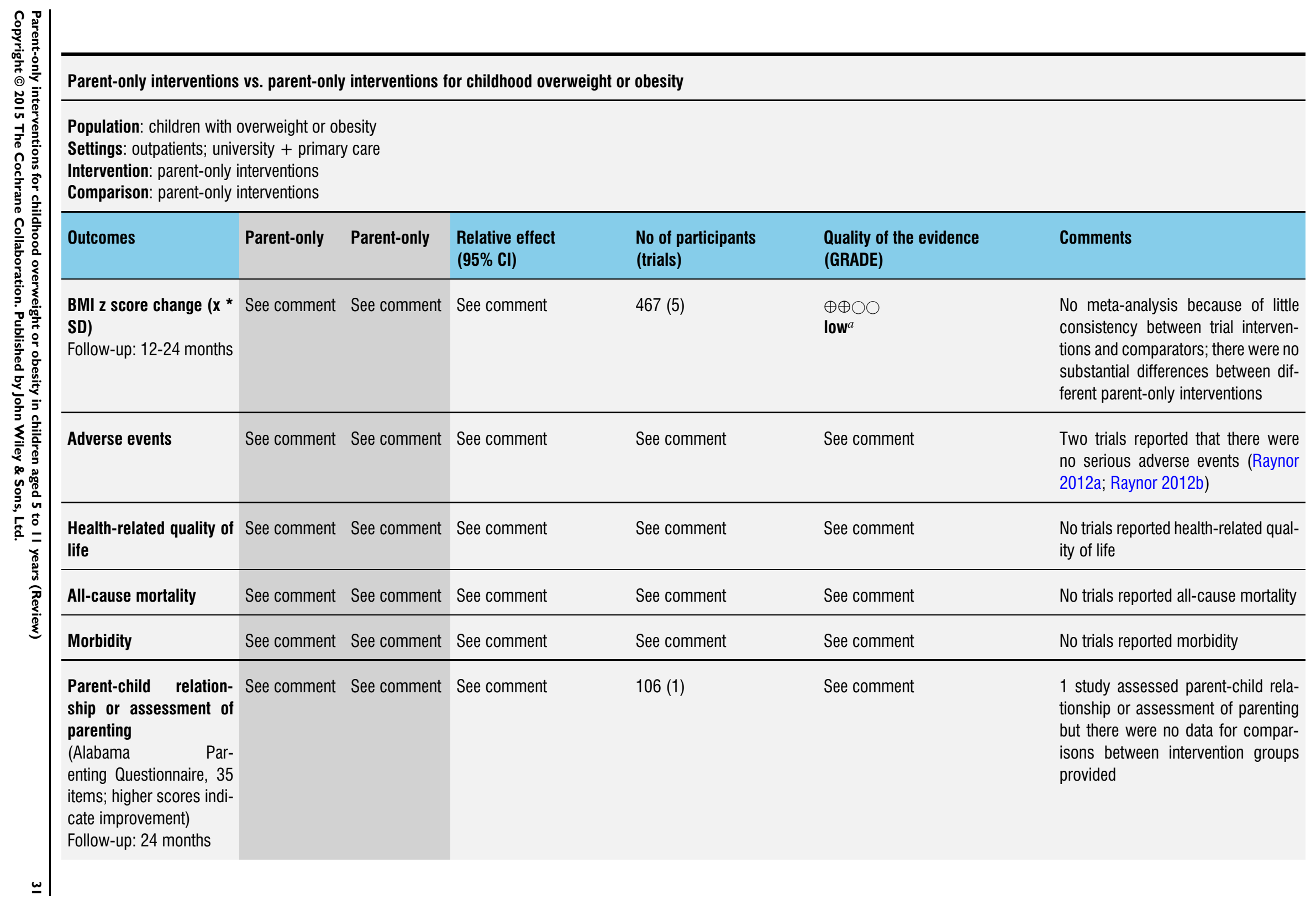




\begin{tabular}{|c|c|c|c|c|}
\hline cioeconomic effects & See comment & See commer & & See comment \\
\hline
\end{tabular}

*The basis for the assumed risk (e.g. the median control group risk across trials) is provided in footnotes. The corresponding risk (and its $95 \%$ confidence interval) is based on the assumed risk in the comparison group and the relative effect of the intervention (and its $95 \% \mathrm{Cl}$ ).

BMI: body mass index; Cl: confidence interval; SD: standard deviation.

GRADE Working Group grades of evidence

High quality: Further research is very unlikely to change our confidence in the estimate of effect.

Moderate quality: Further research is likely to have an important impact on our confidence in the estimate of effect and may change the estimate.

Low quality: Further research is very likely to have an important impact on our confidence in the estimate of effect and is likely to change the estimate. Very low quality: We are very uncertain about the estimate.

" A BMI z score or standard deviation score indicates how many units (of the standard deviation) a child's BMI is above or below the average BMI value for their age group and sex. For instance, a $z$ score of 1.5 indicates that a child' is 1.5 standard deviations above

the average value, and a z score of -1.5 indicates a child is 1.5 standard deviations below the average value" (Noo NHS 2011).

${ }^{a}$ Downgraded by one level because of serious risk of attrition bias and one level for serious imprecision (see Appendix 9) 


\section{DISCUSSION}

\section{Summary of main results}

This systematic review summarised 20 RCTs examining the effect of parent-only interventions for treating overweight and obesity in children aged 5 to 11 years. We only included trials with at least a six-month outcome assessment with the aim of assessing the longer-terms effects of these types of interventions. Interventions and comparators varied between the included trials and we divided the trials into four main groups to ease interpretation. Outcomes assessed also varied between groups; although all trials reported a measure of body weight, this varied between the BMI, BMI z score, BMI percentile and weight. Many trials were of low quality, non-completion rates of the trials were generally high and few trials accounted for these in their analyses. To allow comparisons across trials, we analysed outcome data, where reported, from the post intervention assessment and the longest period of follow-up. Overall, in trials comparing a parent-only intervention with a parent-child intervention there were no substantial differences in BMI measures at either the post intervention follow-up or the longest follow-up period. Other outcomes, reported less consistently across trials, also generally reported no substantial differences between groups, including parental BMI, behavioural changes and health-related quality of life. One study undertook a process evaluation where parents responses about undertaking the programme again were similar across groups. In trials comparing a parent-only intervention with a waiting list control, there was a treatment effect on BMI in favour of the parent-only intervention at the post intervention follow-up and at the longest follow-up period (low quality evidence). There was no substantial effect of the parent-only intervention on parental BMI or the child's self esteem. One trial reported better outcomes in the parent-only groups on a measure of behavioural change and on a measure of parenting discipline practices (low quality evidence). There were no substantial effects of parent-only interventions on BMI or weight when compared with minimal contact control interventions. One trial found reduced levels of parental concern in the parent-only group. Two trials reported process evaluations and the interventions were generally rated more highly by those who responded in the parentonly group than the control group. A number of trials compared a parent-only intervention with another parent-only intervention. To meet the inclusion criteria for the review, parent-only comparisons were required to be a concomitant therapy delivered in the intervention arm. There were few similarities between interventions and comparators across the included trials. Generally, these trials did not show substantial differences between their respective parent-only groups on the various outcomes reported.

All results need to be interpreted cautiously because of the low quality of these trials and the differences between the interventions and comparators.

\section{Overall completeness and applicability of evidence}

This review included only trials that reported outcomes at six months or more; however, within the individual trials there was considerable heterogeneity with the duration of the interventions and their respective follow-ups varied considerably. Few trials had similar intervention characteristics and, together with the mixture of outcomes assessed and results seen, it was difficult to establish whether there is any particular intervention type that is more likely to lead to a successful outcome. It was our intention to explore the impact of the type of parent-only intervention (e.g. focusing on parenting, cognitive behavioural therapy, behaviour change) and the setting (e.g. community, clinic-based, internet), to determine if any specific approach was more effective for the treatment of childhood overweight and obesity. Despite the relatively large number of trials included in the review, the profound clinical heterogeneity meant that we were unable to do this. Few trials provided enough details of the intervention to allow these to be replicated by other trialists (length, type, nature of sessions, group sizes, contents, training of the provider or theoretical basis for the intervention). Few trials reported outcomes over a relatively long period of follow-up (e.g. over two years).

Results seemed to suggest that parent-only interventions are similar to parent-child interventions, and minimal contact interventions, but that they are better than a waiting list control. However, there are numerous issues to consider, not least the sample sizes of many trials, the loss to follow-up and the low quality evidence. There was an insufficient number of trials reporting secondary outcomes of interest to the review and we could only pool BMI indices in meta-analyses.

\section{Quality of the evidence}

Trial quality was generally low with a large proportion of trials being rated at high risk of bias on individual risk of bias criteria, and many others being rated at unclear risk of bias. GRADE assessment of the outcomes that have been pooled in this review have led to trials being downgraded for risk of bias (in particular attrition bias), and also imprecision owing to the small number of trials and small sample sizes. This makes overall interpretation of the data difficult.

\section{Potential biases in the review process}

We conducted a comprehensive search across major databases for interventions involving parent-only interventions. In addition, we screened the reference lists of systematic reviews. Two review authors comprehensively selected, assessed trials; extracted data and assessed quality of trials for inclusion to minimise potential biases in the review processes. No decisions were made about the analysis or investigation of heterogeneity after seeing the data. Where data 
of relevance were missing, either to allow assessment of eligibility or at the data extraction stage, the review authors contacted the study authors for further information. Multiple groups were included in seven of the included studies and for analyses we split the shared group into two or more groups with smaller sample sizes. This approach only partially overcomes a unit of analysis error as the resulting comparisons remain correlated. Three trials are awaiting classification as information is currently unavailable to the review.

\section{Agreements and disagreements with other studies or reviews}

National Institute for Health and Care Excellence (NICE) guidance emphasises the importance of parental support in weight management services for children and young people under the age of 18 years (NICE 2013). However, parent-only interventions are not specifically mentioned, instead family-based interventions are advocated. The results are consistent with a previous metaanalysis that has compared parent-only with parent-child (family-focused) interventions, showing no substantial difference in the change in the BMI z score between groups (Jull 2013). The findings also agree with another systematic review that indicated that parent-only interventions were at least as effective as parentchild interventions, albeit showing higher drop-out from the parent-only interventions (Ewald 2014). Jang 2015 have also considered parent-only interventions in a systematic review using the RE-AIM ( Reach Effectiveness Adoption Implementation Maintenance) framework to analyse reach, adoption, implementation, efficacy/effectiveness and maintenance. They also support the effectiveness of parent-only interventions in improving children's BMIs, but that interventions did not appear to reach higher-risk populations. The current systematic review, as far as we are aware, is the first to analyse the parent-only interventions by comparison group, comparing with parent-child interventions, with waiting list and minimal contact interventions, and with different parentonly interventions.

\section{A U THORS'CONCLUSIONS}

\section{Implications for practice}

Parent-only interventions may be an effective treatment option for overweight or obese children aged 5 to 11 years when compared with waiting list controls; however, parent-only interventions had similar effects compared with parent-child interventions and compared with those with minimal contact controls. However, the evidence for parent-only interventions is at present limited, particularly when the trials were split for analysis by comparator, and some of the trials had a high risk of bias.

\section{Implications for research}

The systematic review identified 10 ongoing trials that have a parent-only intervention arm, which will contribute to the results of this review when being updated. These trials will improve the robustness of the analyses by type of comparator, and may permit subgroup analysis by intervention component and the setting for parent-only interventions.

There is a need to conduct and report cost-effectiveness analyses in these ongoing trials in order to establish whether parent-only interventions are more cost-effective than parent-child interventions. Trial reports should provide adequate details about the interventions to be replicated by others and report important outcomes such as health-related quality of life.

\section{ACK NOW LEDGEMENTS}

We thank Drs Janicke, Moens, Raynor, Roth and Van Grieken for providing additional information about their trials (Janicke 2008; Moens 2012; Munsch 2008; Raynor 2012a; van Grieken 2013). We thank Christine Clar for translating a German publication to confirm its status as an ongoing study (subsequently excluded following contact with the authors). We acknowledge the editorial contributions by Gudrun Paletta and Bernd Richter (Cochrane Metabolic and Endocrine Disorders Group).

\section{R E F E R E N C E S}

\section{References to studies included in this review}

Aragona 1975 \{published data only\}

Aragona J, Cassady J, Drabman RS. Treating overweight

children through parental training and contingency contracting. Journal of Applied Behavior Analysis 1975;8(3): 269-78.

Boutelle 2011 \{published data only\} Boutelle KN, Cafri G, Crow SJ. Parent-only treatment for childhood obesity: a randomized controlled trial. Obesity
2011;19:574-80.

Collins 2011 \{published data only\}

Burrows T, Janet WM, Collins CE. Long-term changes

in food consumption trends in overweight children in the HIKCUPS intervention. Journal of Pediatric Gastroenterology and Nutrition 2011;53(5):543-7. Burrows T, Warren JM, Baur LA, Collins CE. Impact of a child obesity intervention on dietary intake and behaviors. International Journal of Obesity 2008;32(10):1481-8. Burrows T, Warren JM, Collins CE. The impact of a child 
obesity treatment intervention on parent child-feeding practices. International Journal of Pediatric Obesity 2010;5 (1):43-50.

Cliff DP, Okely AD, Morgan PJ, Steele JR, Jones RA, Colyvas K, et al. Movement skills and physical activity in obese children: randomized controlled trial. Medicine and Science in Sports and Exercise 2011;43(1):90-100. Collins CE, Morgan PJ, Okely AD, Burrows TL, Cliff DP, Jones RA, et al. HIKCUPS (Hunter Illawarra Kids Challenge Using Parent Support) reduces BMI z-score up to 2 years: results of a multi-site randomized trial for overweight children. Obesity Reviews 2010;11:280.

* Collins CE, Okely AD, Morgan PJ, Jones RA, Burrows TL, Cliff DP, et al. Parent diet modification, child activity, or both in obese children: an RCT. Pediatrics 2011;127: 619-27.

Jones RA, Okely AD, Collins CE, Morgan PJ, Steele JR, Warren JM, et al. The HIKCUPS trial: a multi-site randomized controlled trial of a combined physical activity skill-development and dietary modification program in overweight and obese children. BMC Public Health. 2007; 7:15.

Okely AD, Collins CE, Morgan PJ, Jones RA, Warren JM, Cliff DP, et al. Multi-site randomized controlled trial of a child-centered physical activity program, a parent-centered dietary-modification program, or both in overweight children: the HIKCUPS study. Journal of Pediatrics 2010; 157(3):388-94.

\section{Esfarjani 2013 \{published data only\}}

Esfarjani F, Khalafi M, Mohammadi F, Mansour A, Roustaee $\mathrm{R}$, Zamani-Nour N, et al. Family-based intervention for childhood obesity: an experience among Tehranian children. Annals of Nutrition and Metabolism 2013;63:844. * Esfarjani F, Khalafi M, Mohammadi F, Mansour A, Roustaee R, Zamani-Nour N, et al. Family-based intervention for controlling childhood obesity: an experience among Iranian children. International Journal of Preventive Medicine 2013;4(3):358-65.

Estabrooks 2009 \{published data only\} Estabrooks PA, Shoup JA, Gattshall M, Dandamudi P, Shetterly S, Xu S. Automated telephone counseling for parents of overweight children: a randomized controlled trial. American Journal Preventive Medicine 2009;26(1): $35-42$.

\section{Golan 2006 \{published data only\}}

Golan M. Parents as agents of change in childhood obesity from research to practice. International Journal of Pediatric Obesity 2006;1:66-76.

* Golan M, Kaufman V, Shahar DR. Childhood obesity treatment: targeting parents exclusively v. parents and children. British Journal of Nutrition 2006;95:1008-15.

Golley 2007 \{published data only\}

* Golley RK, Magarey AM, Baur LA, Steinbeck KS, Daniels LA. Twelve-month effectiveness of a parent-led, familyfocused weight-management program for prepubertal children: a randomized, controlled trial. Pediatrics 2007; 119(3):517-25.

Golley RK, Magarey AM, Daniels LA. Children's food and activity patterns following a six-month child weight management program. International Journal of Pediatric Obesity 2011;6:409-14.

Janicke 2008 \{published data only\}

* Janicke DM, Sallinen BJ, Perri MG, Lutes LD, Huerta $\mathrm{M}$, Silverstein JH, et al. Comparison of parent-only versus family-based interventions for overweight children in underserved rural settings: outcomes from Project STORY. Archives of Pediatrics and Adolescent Medicine 2008;162:

1119-25.

Janicke DM, Sallinen BJ, Perri MG, Lutes LD, Silverstein JH, Brumback B. Comparison of program costs for parentonly and family-based interventions for pediatric obesity in medically underserved rural settings. Journal of Rural Health 2009;25:326-30.

Janicke DM, Sallinen BJ, Perri MG, Lutes LD, Silverstein JH, Huerta MG, et al. Sensible treatment of obesity in rural youth (STORY): design and methods. Contemporary Clinical Trials 2008;29(2):270-80.

Walker K. Mechanisms of self-esteem change in overweight children participating in a family-based weight management program. Dissertation. University of Florida. 2007.

\section{Jansen 2011 \{published data only\}}

Jansen E, Mulkens S, Jansen A. Tackling childhood overweight: treating parents exclusively is effective. International Journal of Obesity (Lond) 2011;35(4):501-9.

Magarey 2011 \{published data only\}

Magarey AM, Perry RA, Baur LA, Steinbeck KS, Sawyer M, Hills AP, et al. A parent-led family-focused treatment program for overweight children aged 5 to 9 years: the PEACH RCT. Pediatrics 2011;127(2):214-22.

\section{Mazzeo 2014 \{published data only\}}

Bean MK, Wilson DB, Thornton LM, Kelly N, Mazzeo SE. Dietary intake in a randomized-controlled pilot of NOURISH: a parent intervention for overweight children. Preventive Medicine 2012;55(3):224-7.

* Mazzeo SE, Kelly NR, Stern M, Gow RW, Cotter EW, Thornton LM, et al. Parent skills training to enhance weight loss in overweight children: evaluation of NOURISH. Eating Behavior 2014;15(2):225-9.

Mazzeo SE, Kelly NR, Stern M, Gow RW, Serdar K, Evans RK, et al. Nourishing Our Understanding of Role Modeling to Improve Support and Health (NOURISH): design and methods. Contemporary Clinical Trials 2012;33(3):515-22.

\section{Moens 2012 \{published data only\}}

Moens E, Braet C. Training parents of overweight children in parenting skills: a 12-month evaluation. Behaviour. 2012;40(1):1-18.

Munsch 2008 \{published data only\}

* Munsch S, Roth B, Michael T, Meyer AH, Biedert E, Roth $\mathrm{S}$, et al. Randomized controlled comparison of two cognitive behavioral therapies for obese children: 
mother versus mother-child cognitive behavioral therapy. Psychotherapy and Psychosomatics 2008;77:235-46. Roth B, Munsch S, Meyer AH. Long-term evaluation of a psychological training for obese children and their parents (TAKE) [Langzeitevaluation eines psychologischen Trainingsfür adipöse Kinder und ihre Eltern (TAKE)]. Praxis der Kinderpsychologie und Kinderpsychiatrie 2011;60: 304-21.

Raynor 2012a \{published data only\}

Raynor HA, Osterholt KM, Hart CN, Jelalian E, Vivier P, Wing RR. Efficacy of US pediatric obesity primary care guidelines: two randomized trials. Pediatric Obesity 2012;7 (1):28-38

Raynor 2012b \{published data only\}

Raynor HA, Osterholt KM, Hart CN, Jelalian E, Vivier P, Wing RR. Efficacy of US pediatric obesity primary care guidelines: two randomized trials. Pediatric Obesity 2012;7 (1):28-38

Resnick 2009 \{published data only\}

Resnick EA, Bishop M, O'Connell A, Hugo B, Isern G, Timm A, et al. The CHEER study to reduce BMI in Elementary School students: a school-based, parentdirected study in Framingham, Massachusetts. Journal of School Nursing 2009;25(5):361-72.

Resnicow 2015 \{published data only\} Resnicow K, McMaster F, Bocian A, Harris D, Zhou Y, Snetselaar L, et al. Motivational nterviewing and dietary counseling for obesity in primary care: an RCT. Pediatrics 2015;135:649-57.

Small 2013 \{published data only\} Small L, Bonds-McClain D. A primary care-based randomized treatment trial with overweight/obese children. Communicating Nursing Research 2013;46:291.

* Small L, Bonds-McClain D, Melnyk B, Vaughan L, Gannon AM. The preliminary effects of a primary carebased randomized treatment trial with overweight and obese young children and their parents. Journal of Pediatric Health Care 2014;28(3):198-207.

van Grieken 2013 \{published data only\} Veldhuis L, Struijk MK, Kroeze W, Oenema A, Renders $\mathrm{CM}$, Bulk-Bunschoten AM, et al. "Be active, eat right", evaluation of an overweight prevention protocol among 5year-old children: design of a cluster randomised controlled trial. BMC Public Health 2009;9:177.

van Grieken A, Renders CM, Veldhuis L, Looman CW, Hirasing RA, Raat H. Promotion of a healthy lifestyle among 5-year-old overweight children: health behavior outcomes of the 'Be active, eat right' study. BMC Public Health 2014;14:59.

* van Grieken A, Veldhuis L, Renders CM, Borsboom GJ, van der Wouden JC, Hirasing RA, et al. Population-based childhood overweight prevention: outcomes of the 'Be Active, Eat Right' study. PLoS One 2013;8:5.

West 2010 \{published data only\}

West F, Sanders MR, Cleghorn GJ, Davies PS. Randomised clinical trial of a family-based lifestyle intervention for childhood obesity involving parents as the exclusive agents of change. Behaviour Research and Therapy 2010;48(12): 1170-9.

\section{References to studies excluded from this review}

\section{Berry 2007 \{published data only\}}

Berry D, Savoye M, Melkus G, Grey M. An intervention for multiethnic obese parents and overweight children. Applied Nursing Research 2007;20(2):63-71.

\section{Biotnott 2009 \{published data only\}}

Boitnott AD. Childhood obesity: development of a parentfocused intervention. Dissertation Abstracts International: Section B: The Sciences and Engineering 2009;7(3):1588.

Bohlin 2012 \{published data only\}

Bohlin A, Klaesson S, Kowalski J. Can telephone consultations substitute visits in treatment of childhood obesity? Results from a randomized trial. Obesity Facts 2012;5:237.

De Bock 2013 \{published data only\} De Bock F, Fischer JE, Hoffmann K, Renz-Polster H. A participatory parent-focused intervention promoting physical activity in preschools: design of a clusterrandomized trial. BMC Public Health 2010;10:49.

* De Bock F, Genser B, Raat H, Fischer JE, Renz-Polster H. A participatory physical activity intervention in preschools: a cluster randomized controlled trial. American Journal of Preventive Medicine 2013;45(1):64-74.

Dewes 2014 \{published data only\} Dewes O, Sluyter J, Scragg R, Jiang Y, Percival T. Fanau FAB: parent-focused weight management programme for Pacific children. Obesity Reviews 2014;15:212.

Hendrie 2011 \{published data only\}

Hendrie GA, Golley RK. A parent led intervention targeting purchase of low fat dairy foods reduces saturated fat intake without a change in energy, BMI or negative food consequences. Obesity Research and Clinical Practice 2011;5: S28.

Hystad 2013 \{published data only\} Hystad HT, Steinsbekk S, Ødegard R, Wichstrøm L, Gudbrandsen OA. A randomised study on the effectiveness of therapist-led v. self-help parental intervention for treating childhood obesity. British Journal of Nutrition 2013;110: 1143-50.

John 2009 \{unpublished data only\} John R. Effects of Parent-Focused Media Interventions on Body Mass Index, Waist Size, Self-Perception, Family Eating Habits, and Family Activity Habits in Overweight Hispanic Children [Thesis]. New York: Teachers College, Columbia University, 2009.

Lawson 2015 \{published data only\} Lawson Health Research. FOR HEALTH: a familyoriented healthy eating, activity and lifestyle intervention for overweight preschool children, 2015. ClinicalTrials.gov/ show/NCT01698606 (accessed 16 December 2015). 
Le Gross 2006 \{published data only\}

The "Kids on Track" program, 2006. www.anzctr.org.au/ ACTRN12606000382572.aspx (accessed 15 December 2015).

NHLBI 2008 \{published data only\}

National Heart Lung Blood Institute. Family-based nutrition intervention for Latino children, 2008. ClinicalTrials.gov/show/NCT00224887 (accessed 15 December 2015).

Parra-Medina 2015 \{published data only\} University of Texas Health Science Center. Health4Kids intervention trial for Hispanic families, 2015. ClinicalTrials.gov/show/NCT02343367 (accessed 15 December 2015).

Shelton 2007 \{published data only\} Shelton D, LeGros K, Norton L, Stanton-Cook S, Morgan J, Masterman P. Randomised controlled trial: a parentbased group education programme for overweight children. Journal of Paediatrics and Child Health 2007;43:799-805.

Steele 2014 \{published data only\}

Steele RG, Jensen CD, Gayes LA, Liebold HC. Medium is the message: moderate parental control of feeding correlates with improved weight outcome in a pediatric obesity intervention. Journal of Pediatric Psychology 2014;39(7): 708-17.

Volkenant 2011 \{published data only\}

Volkenant KR, Quinlan NP, Ubinger M, Rukstalis M, Cochran WJ. Early childhood obesity intervention in primary care: parent stress levels and child mental health symptoms. Obesity 2011;19:S108.

Warschburger 2013 \{published data only\}

* Warschburger P, Kroller K, Unverzagt S, Haerting J. What is the parents' part in long-term weight management of their obese child? Results from the EPOC study. Obesity Facts 2013;6:230.

Warschburger P, Kroller K, Unverzagt S, Haerting J, van Egmond-Fröhlich A. Empowering parents of obese children (EPOC): a randomized-controlled trial on additional longterm weight effects of a parent training. Submitted data. Warschburger P, Kuhne D, Kroller K. Do parents influence the long term development of obese children's weight? First results of the EPOC-study [in German]. Padiatrische Praxis 2012;79(2):185-92.

\section{References to studies awaiting assessment}

\section{Geronilla 1981 \{published and unpublished data\}}

Geronilla LS. A study of weight control in pediatric obesity using mothers as behavior modifiers. Dissertation Abstracts International 1981;42(5-B):2027.

Gillick 1975 \{published and unpublished data\} Gillick SL. Training parents as therapists in the treatment of juvenile obesity. Dissertation Abstracts International 1975;35 (10-B):5111-2.

\section{Golan 1998 \{published data only\}}

Golan M, Crow S. Targeting parents exclusively in the treatment of childhood obesity: long-term results. Obesity Research 2004;12:357-61.

* Golan M, Fainaru M, Weizman A. Role of behaviour modification in the treatment of childhood obesity with the parents as the exclusive agents of change. International journal of obesity and related metabolic disorders 1998;22: 1217-24.

Golan M, Weizman A, Apter A, Fainaru M. Parents as the exclusive agents of change in the treatment of childhood obesity. American Journal of Clinical Nutrition 1998;67(6): 1130-5.

Golan M, Weizman A, Fainaru M. Impact of treatment for childhood obesity on parental risk factors for cardiovascular disease. Preventive Medicine 1999;29(6):519-26.

\section{References to ongoing studies}

\section{Ball 2012 \{published data only\}}

Ball GD, Ambler KA, Keaschuk RA, Rosychuk RJ, Holt NL, Spence JC, et al. Parents as agents of change (PAC) in pediatric weight management: the protocol for the PAC randomized clinical trial. BMC Pediatrics 2012;12:114. [DOI: 10.1186/1471-2431-12-114.]

Dalton 2011 \{published data only\} Dalton WT, Schetzina KE, Holt N, Fulton-Robinson H, Ho AL, Tudiver F, et al. Parent-Led Activity and Nutrition (PLAN) for healthy living: design and methods. Contemporary Clinical Trials 2011;32(6):882-92.

Gerards 2012 \{published data only\} Gerards SM, Dagnelie PC, Jansen MW, van der Goot LO, de Vries NK, Sanders MR, et al. Lifestyle Triple P: a parenting intervention for childhood obesity. BMC Public Health 2012;12:267. [DOI: 10.1186/1471-2458-12-267]

\section{Janicke 2011 \{published data only\}} Janicke DM, Lim CS, Perri MG, Bobroff LB, Mathews AE, Brumback BA, et al. The Extension Family Lifestyle Intervention Project (E-FLIP for kids): design and methods. Contemporary Clinical Trials 2011;32(1):50-8. [DOI: 10.1016/j.cct.2010.08.002]

\section{NCT01197443 \{published data only\}} Acronym: PAAC. Ongoing study Study start date: November 2010Study completion date: July 2015.

NCT01546727 \{published data only\} Acronym: Behavioral Treatment for Obese Preschoolers (LAUNCH). Ongoing study Study start date: March 2012Study completion date: November 2016.

NCT01552642 \{published data only\} Acronym: none. Ongoing study Study start date: February 2013Study completion date: August 2015.

\section{NCT01792531 \{published data only\}} Acronym: More and Less study $(\mathrm{M}+\mathrm{L})$. Ongoing study Study start date: January 2013Study completion date: December 2017. 


\section{NCT02373670 \{published data only\}}

Acronym: none. Ongoing study Study start date: January 2015Study completion date: June 2015.

Önnerfält 2012 \{published data only\}

Önnerfält J, Erlandsson LK, Orban K, Broberg M, Helgason C, Thorngren-Jerneck K. A family-based intervention targeting parents of preschool children with overweight and obesity: conceptual framework and study design of LOOPS - Lund Overweight and Obesity Preschool Study. BMC Public Health 2012;12:879.

\section{Additional references}

\section{Beller 2013}

Beller EM, Chen JK, Wang UL, Glasziou PP. Are systematic reviews up-to-date at the time of publication?. Systematic Reviews 2013;2(1):36. [2046-4053: (Electronic)]

\section{Bocca 2013}

Bocca G, Ongering EC, Stolk RP, Sauer PJ. Insulin resistance and cardiovascular risk factors in 3- to 5-year old overweight or obese children. Hormone Research in Paediatrics 2013;80(3):201-6.

\section{Clark 2007}

Clark HR, Goyder E, Bissell P, Blank L, Peters J. How do parents' child feeding behaviours influence child weight? Implications for childhood obesity policy. Journal of Public Health 2007;29:132-41.

\section{CMO 2012}

Chief Medical Officer. Annual report of the Chief Medical Officer: surveillance volume, 2012: on the State of the Public's Health. www.gov.uk/government/publications/ chief-medical-officer-annualreport-surveillance-volume2012 (accessed 30 March 2015).

\section{Cole 2000}

Cole TJ, Bellizzi MC, Flegal KM, Dietz WH. Establishing a standard definition for child overweight and obesity worldwide: international survey. $B M J$ 2000;320(7244): $1240-3$.

\section{Cole 2012}

Cole TJ, Lobstein T. Extended international (IOTF) body mass index cut-offs for thinness, overweight and obesity. Pediatric Obesity 2012;7(4):284-94.

\section{Daniels 2009}

Daniels SR. Complications of obesity in children and adolescents. International Journal of Obesity 2009;33(Suppl 1):S60-5.

\section{de Onis 2010}

de Onis M, Blössner M. Borghi E. Global prevalence and trends of overweight and obesity among preschool children. American Journal of Clinical Nutrition 2010;92(5):1257-64.

Eady 2008

Eady AM, Wilczynski NL, Haynes RB. PsycINFO search strategies identified methodologically sound therapy studies and review articles for use by clinicians and researchers. Journal of Clinical Epidemiology 2008;61(1):34-40.
Egan 2013

Egan K, Ettinger A, Bracken M. Childhood body mass index and subsequent physician-diagnosed asthma: a systematic review and meta-analysis of prospective cohort studies. BMC Pediatrics 2013;13(1):121.

\section{Egbewale 2014}

Egbewale BE, Lewis M, Sim J. Bias, precision and statistical power of analysis of covariance in the analysis of randomized trials with baseline imbalance: a simulation study. $B M C$ Medical Research Methodology 2014;14:49:49. [DOI: 10.1186/1471-2288-14-49]

\section{Ells 2015}

Ells LJ, Hancock C, Copley VR, Mead E, Dinsdale H, Kinra S, et al. Prevalence of severe childhood obesity in England: 2006-2013. Archives of Disease in Childhood 2015;100(7):631-6.

\section{Ewald 2014}

Ewald H, Kirby J, Rees K, Robertson W. Parent-only interventions in the treatment of childhood obesity: a systematic review of randomized controlled trials. Journal of Public Health 2014;36(3):476-89.

Faith 2012

Faith MS, Van Horn L, Appel LJ, Burke LE, Carson JAS, Franch HA, et al. AHA scientific statement: evaluating parents and adult caregivers as "Agents of Change" for treating obese children: evidence for parent behavior change strategies and research gaps: a scientific statement from the American Heart Association. Circulation 2012;125: 1186-207.

Follman 1992

Follmann D, Elliot P, Suh I, Cutler J. Variance imputation for overviews of clinical trials with continuous response. Journal of Clinical Epidemiology 1992;45(7):769-73.

Freedman 2006

Freedman DS, Khan LK, Serdula MK, Ogden CL, Dietz WH. Racial and ethnic differences in secular trends for childhood BMI, weight, and height. Obesity 2006;14(2): 301-8.

Glenny 1997

Glenny AM, O'Meara S, Melville A, Sheldon TA, Wilson C. The treatment and prevention of obesity: a systematic review of the literature. International Journal of Obesity and Related Metabolic Disorders 1997;21(9):715-37.

\section{Golan 2004}

Golan M, Crow S. Targetting parents exclusively in the treatment of childhood obesity: long-term results. Obesity Research 2004;12:357-61.

\section{Griffiths 2010}

Griffiths LJ, Parsons TJ, Hill AJ. Self-esteem and quality of life in obese children and adolescents: a systematic review. International Journal of Pediatric Obesity 2010;5(4): 282-304.

\section{Gustafson 2006} Gustafson SL, Rhodes RE. Parental correlates of physical activity in children and early adolescents. Sports Medicine 2006;36:79-97. 


\section{Halliday 2014}

Halliday JA, Palma CL, Mellor D, Green J, Renzaho AMN. The relationship between family functioning and child and adolescent overweight and obesity: a systematic review. International Journal of Obesity 2014;38:480-93.

Higgins 2002

Higgins JPT, Thompson SG. Quantifying heterogeneity in a meta-analysis. Statistics in Medicine 2002;21 (11):1539-58.

\section{Higgins 2003}

Higgins JPT, Thompson SG, Deeks JJ, Altman DG. Measuring inconsistency in meta-analyses. BMJ 2003;327 (7414):557-60.

\section{Higgins 2009}

Higgins JPT, Thompson SG, Spiegelhalter DJ. A reevaluation of random-effects meta-analysis. Journal of the Royal Statistical Society. Series A (Statistics in Society) 2009; 172(1):137-59.

\section{Higgins 2011a}

Higgins JPT, Green S (editors). Cochrane Handbook for Systematic Reviews of Interventions Version 5.1.0 [updated March 2011]. The Cochrane Collaboration, 2011. Available from www.cochrane-handbook.org.

\section{Higgins 2011b}

Higgins JPT, Altman DG, Gøtzsche PC, Jüni P, Moher D, Oxman AD, et al. The Cochrane Collaboration's tool for assessing risk of bias in randomised trials. BMJ 2011;343: d5928.

Hróbjartsson 2013

Hróbjartsson A, Thomsen AS, Emanuelsson F, Tendal B, Hilden J, Boutron I, et al. Observer bias in randomized clinical trials with measurement scale outcomes: a systematic review of trials with both blinded and nonblinded assessors. Canadian Medical Association Journal 2013;185 (4):E201-11.

\section{HSCIC 2015}

Health and Social Care Information Centre. National Child Measurement Programme - England, 2012-13 school year. www.hscic.gov.uk/catalogue/PUB13115 (accessed 30 March 2015).

Jang 2015

Jang M, Chao A, Whittemore R. Evaluating intervention programs targeting parents to manage childhood overweight and obesity: a systematic review using the RE-AIM framework. Journal of Pediatric Nursing 2015;30(6): 877-87. [DOI: 10.1016/j.pedn.2015.05.004.]

Jull 2013

Jull A, Chen R. Parent-only vs. parent-child (familyfocused) approaches for weight loss in obese and overweight children: a systematic review and meta-analysis. Obesity Reviews 2013;14:761-8.

Kelly 2013

Kelly AS, Barlow SE, Rao G, Inge TH, Hayman LL, Steinberger J, et al. Severe obesity in children and adolescents: identification, associated health risks, and treatment approaches: a scientific statement from the
American Heart Association. Circulation 2013;128(15):

1689-712.

\section{Kirkham 2010}

Kirkham JJ, Dwan KM, Altman DG, Gamble C, Dodd $\mathrm{S}$, Smyth R, et al. The impact of outcome reporting bias in randomised controlled trials on a cohort of systematic reviews. BMJ 2010;340:c365. [DOI: 10.1136/bmj.c365]

\section{Knai 2012}

Knai C, Lobstein T, Darmon N, Rutter H, McKee M. Socioeconomic patterning of childhood overweight status in Europe. International Journal of Environmental Research and Public Health 2012;9(4):1472-89.

\section{Leclercq 2013}

Leclercq E, Leeflang MM, van Dalen EC, Kremer LC. Validation of search filters for identifying pediatric studies in PubMed. Journal of Pediatrics 2013;162(3):629-34.

\section{Liberati 2009}

Liberati A, Altman DG, Tetzlaff J, Mulrow C, Gøtzsche PC, Ioannidis JPA, et al. The PRISMA statement for reporting systematic and meta-analyses of studies that evaluate interventions: explanation and elaboration. PLoS Medicine 2009;6(7):1-28. [DOI: 10.1371/journal.pmed.1000100]

\section{Lobstein 2004}

Lobstein T, Baur L, Uauy R. Obesity in children and young people: a crisis in public health. Obesity Reviews 2004;5 (Suppl 1):4-104.

\section{Meader 2014}

Meader N, King K, Llewellyn A, Norman G, Brown J, Rodgers $\mathrm{M}$, et al. A checklist designed to aid consistency and reproducibility of GRADE assessments: development and pilot validation. Systemic Reviews 2014;3:82.

\section{Narang 2012}

Narang I, Mathew JL. Childhood obesity and obstructive sleep apnea. Journal of Nutrition and Metabolism 2012; 2012:134202. [DOI: $10.1155 / 2012 / 134202$; PUBMED: 22957216]

\section{$\mathrm{Ng} 2014$}

Ng M, Fleming T, Robinson M, Thomson B, Graetz N, Margono C, et al. Global, regional, and national prevalence of overweight and obesity in children and adults during 1980-2013: a systematic analysis of the Global Burden of Disease Study 2013. Lancet 2014;384(9945):766-81.

\section{NICE 2013}

National Institute for Health and Care Excellence. Managing overweight and obesity among children and young people: lifestyle weight management services. NICE Guidelines PH47, 2013. www.nice.org.uk/guidance/ph47 (accessed 15 December 2015).

\section{Noo NHS 2011}

National Obesity Observatory on behalf of the Public Health Observatories in England. A simple guide to classifying body mass index in children, 2011. www.noo.org.uk/uploads/doc/ vid $11601{ }^{\prime}$ A' simple guide to 'classifying ${ }^{\prime} \mathrm{BMI}$ ' in ' children.pdf (accessed 3 November 2015). 
Ogden 2006

Ogden J, Reynolds R, Smith A. Expanding the concept of parental control: a role for overt and covert control in children's snacking behaviour?. Appetite 2006;47:100-6.

\section{Olds 2011}

Olds T, Maher C, Zumin S, Péneau S, Lioret S, Castetbon $\mathrm{K}$, et al. Evidence that the prevalence of childhood overweight is plateauing: data from nine countries. International Journal of Pediatric Obesity 2011;6(5-6): 342-60.

Oude Luttikhuis 2009

Oude Luttikhuis H, Baur L, Jansen H, Shrewbury VA, O'Malley C, Stolk RP, et al. Interventions for treating obesity in children. Cochrane Database of Systematic Reviews 2009, Issue 1. [DOI: 10.1002/ 14651858.CD001872.pub2]

\section{Parsons 1999}

Parsons TJ, Power C, Logan S, Summerbell CD. Childhood predictors of adult obesity: a systematic review. International Journal of Obesity 1999;23(Suppl 8):S1-107.

\section{Paulis 2014}

Paulis WD, Silva S, Koes BW, van Middelkoop M. Overweight and obesity are associated with musculoskeletal complaints as early as childhood: a systematic review. Obesity Reviews 2014;15(1):52-67.

Puhl 2007

Puhl RM, Latner JD. Stigma, obesity, and the health of the nation's children. Psychology Bulletin 2007;133(4):557-80.

\section{Rajput 2014}

Rajput N, Tuohy P, Mishra S, Smith A, Taylor B. Overweight and obesity in 4-5-year-old children in New Zealand: results from the first 4 years (2009-2012) of the B4 School Check programme. Journal of Paediatrics and Child Health 2014;51(3):334-43.

\section{Reilly 2003}

Reilly JJ, Methven E, McDowell ZC, Hacking B, Alexander D, Stewart L, et al. Health consequences of obesity. Archives of Diseases in Childhood 2003;88(9):748-52.

\section{Reilly 2011}

Reilly JJ, Kelly J. Long-term impact of overweight and obesity in childhood and adolescence on morbidity and premature mortality in adulthood: systematic review. International Journal of Obesity 2011;35(7):891-8.

Riley 2011

Riley RD, Higgins JP, Deeks JJ. Interpretation of random effects meta-analyses. BMJ 2011;342:d549.

\section{Rokholm 2010}

Rokholm B, Baker JL, Sørenson TI. The levelling off of the obesity epidemic since the year 1999 - a review of evidence and perspectives. Obesity Reviews 2010;11(12):835-46.

\section{Shrewsbury 2008}

Shrewsbury V, Wardle J. Socioeconomic status and adiposity in childhood: a systematic review of cross-sectional studies 1990-2005. Obesity (Silver Spring) 2008;16(2):275-84.

\section{Singh 2008}

Singh AS, Mulder C, Twisk JW, vanMechelen W, Chinapaw MJ. Tracking of childhood overweight into adulthood: a systematic review of the literature. Obesity Reviews 2008;9 (5):474-88

\section{Skinner 2014}

Skinner AC, Skelton JA. Prevalence and trends in obesity and severe obesity among children in the United States, 1999-2012. JAMA Pediatrics 2014;168(6):561-6.

\section{Sleddens 2011}

Sleddens EF, Gerards SM, Thijs C, de Vries NK, Kremers SP. General parenting, childhood overweight and obesityinducing behaviors: a review. International Journal of Pediatric Obesity 2011;6:e12-27.

\section{Sterne 2011}

Sterne JA, Sutton AJ, Ioannidis JP, Terrin N, Jones DR, Lau $\mathrm{J}$, et al. Recommendations for examining and interpreting funnel plot asymmetry in meta-analyses of randomised controlled trials. BMJ 2011;343:d4002.

Tang-Peronard 2008

Tang-Peronard JL, Heitmann BL. Stigmatization of obese children and adolescents, the importance of gender. Obesity Reviews 2008;9(6):522-34.

Upton 2012

Upton D, Upton P, Bold J, Peters DM. Regional evaluation of weight management programmes for children and families, 2012. www.obesitywm.org.uk/resources/ Worcester' Report Fina.pdf (accessed 15 December 2015).

Wang 2012

Wang Y, Lim H. The global childhood obesity epidemic and the association between socio-economic status and childhood obesity. International Review of Psychiatry 2012; 24(3):176-88.

\section{Wansink 2006}

Wansink B. Nutritional gatekeepers and the $72 \%$ solution. Journal of the American Dietetic Association 2006;106: 1324-7.

\section{Whitaker 1997}

Whitaker RC, Wright JA, Pepe MS, Seidel KD, Dietz WH. Predicting obesity in young adulthood from childhood and parental obesity. New England Journal of Medicine 1997; 337(13):869-73.

\section{WHO 2015a}

World Health Organization. Fact sheet on overweight and obesity. www.who.int/mediacentre/factsheets/fs311/en (accessed 30 March 2015).

\section{WHO 2015b}

World Health Organization. The WHO Child Growth Standards. www.who.int/childgrowth/publications/ technical.report.pub/en/ (accessed 30 March 2015).

\section{Wong 2006a}

Wong SS, Wilczynski NL, Haynes RB. Developing optimal search strategies for detecting clinically sound treatment studies in EMBASE. Journal of the Medical Library Association 2006;94(1):41-7. 
Wong 2006b

Wong SS, Wilczynski NL, Haynes RB. Optimal CINAHL search strategies for identifying therapy studies and review articles. Journal of Nursing Scholarship 2006;38(2):194-9.

\section{Wood 2008}

Wood L, Egger M, Gluud LL, Schulz KF, Juni P, Altman DG, et al. Empirical evidence of bias in treatment effect estimates in controlled trials with different interventions and outcomes: meta-epidemiological study. BMJ 2008;336
(7644):601-5.

\section{References to other published versions of this review}

\section{Summerbell 2003}

Summerbell CD, Ashton V, Campbell KJ, Edmunds L, Kelly S, Waters E. Interventions for treating obesity in children. Cochrane Database of Systematic Reviews 2003, Issue 3. [DOI: 10.1002/14651858.CD001872]

* Indicates the major publication for the study 


\section{CHARACTERISTICS OF STUDIES}

\section{Characteristics of included studies [author-defined order]}

Resnicow 2015

Cluster randomised controlled trial
Randomisation ratio: initially $1: 1: 1$, final 5 sites randomised $1: 2: 2$ owing to higher
drop-out in the practices providing the 2 interventions
Superiority design

Participants

Inclusion criteria: aged 2-8 years with a BMI $\geq 85$ th and $\leq 97$ th percentile based on Centers for Disease Control cut-points (reference provided)

Exclusion criteria: type 1 or type 2 diabetes, non-English-speaking parent, no working telephone, chronic medical disorders, chromosomal disorders, syndromes and nonambulatory conditions, medications known to affect growth, enrolment in a weight loss programme, seen by weight loss specialist in past 12 months

Diagnostic criteria: as above

Interventions

Number of study centres: -

Treatment before study: -

Titration period: -

Description of interventions:

BMI2 (Brief Motivational Interviewing to reduce body mass index):

1. Moderate-intensity ( 4 sessions, 3 in year 1 ) primary care providers (PCP) motivational interviewing (MI) based counselling. PCPs received 2 days of in-person training in MI and behaviour therapy and an interactive MI DVD training. Provided counselling sessions with a parent of the index child in year 1 and 1 additional "booster" visit in year 2 as well as usual care (described below). MI uses specific techniques such as reflective listening, autonomy support, shared decision-making, and eliciting change talk. Focused on discrete behaviours, such as snack foods, sweetened beverages, fruits, vegetables, TV/screen time and physical activity/exercise. Provided positive feedback for healthy behaviours and then, collaboratively with the parent, identify behaviours that might be modified. Pre-existing or new materials written in a style consistent with MI and self determination theory. Content emphasised child choice in making behaviour change. Self monitoring logs could be used, clinicians educational materials and logs specific to the family

2. High-Intensity, PCP and dietician, intervention. Same intervention components as moderate-intensity group ( 4 sessions with PCP) but added 6 MI-based counselling (4 in year 1) from a trained dietician. Sessions were delivered either in-person or by telephone. Dieticians given same training in $\mathrm{MI}$ as the PCPs

3. Usual care: routine care by the PCP, as well as standard educational materials for parents on healthy eating and exercise. Practitioners attended a half-day orientation session that included current treatment guidelines

PCPs and dieticians were incentivised for the number of sessions and the number of participants recruited additional payments for retaining $50 \%$ and $80 \%$ of cohort 
Resnicow 2015 (Continued)

\begin{tabular}{ll} 
Study details & $\begin{array}{l}\text { Run-in period: - } \\
\text { Study terminated before regular end (for benefit/because of adverse events): no }\end{array}$ \\
\hline Publication details & $\begin{array}{l}\text { Language of publication: English } \\
\text { Non-commercial funding } \\
\text { Publication status: peer-reviewed journal }\end{array}$ \\
\hline
\end{tabular}

Stated aim for study Quote from publication: "to test the efficacy of moderate-intensity (4 sessions) PCP MIbased counselling and the effect of adding 6 MI-based counselling sessions by trained dietitians delivered to parents of overweight youth aged 2 to 8 years recruited through primary care offices"

Notes

Risk of bias

\begin{tabular}{|c|c|c|}
\hline Bias & Authors' judgement & Support for judgement \\
\hline $\begin{array}{l}\text { Random sequence generation (selection } \\
\text { bias) }\end{array}$ & Unclear risk & $\begin{array}{l}\text { Quote from publication: "randomly as- } \\
\text { signed" } \\
\text { Comment: no details }\end{array}$ \\
\hline Allocation concealment (selection bias) & Unclear risk & Comment: no details \\
\hline $\begin{array}{l}\text { Blinding of participants and personnel } \\
\text { (performance bias) } \\
\text { Objective outcomes }\end{array}$ & High risk & $\begin{array}{l}\text { Quote from publication: “open label” } \\
\text { Comment: investigator-assessed }\end{array}$ \\
\hline $\begin{array}{l}\text { Blinding of outcome assessment (detection } \\
\text { bias) } \\
\text { Objective outcome }\end{array}$ & Low risk & $\begin{array}{l}\text { Quote from publication: "open label" } \\
\text { Comment: investigator-assessed, low risk } \\
\text { of bias from objective outcomes }\end{array}$ \\
\hline $\begin{array}{l}\text { Incomplete outcome data (attrition bias) } \\
\text { Objective outcomes }\end{array}$ & Low risk & $\begin{array}{l}\text { Comment: reported with reasons per prac- } \\
\text { tice }\end{array}$ \\
\hline Selective reporting (reporting bias) & High risk & $\begin{array}{l}\text { Comment: no secondary outcomes re- } \\
\text { ported }\end{array}$ \\
\hline Other bias & Low risk & Comment: no other bias \\
\hline
\end{tabular}

\section{Mazzeo 2014}

Methods

Parallel randomised controlled trial

Randomisation ratio: not reported

Superiority design

Parent-only interventions for childhood overweight or obesity in children aged 5 to I I years (Review) 
Participants

Interventions
Inclusion criteria: $\mathrm{BMI} \geq$ the 85 th percentile who primarily resided with the participating carer. Participants also had to speak English, be able to understand basic instructions and perform simple exercises

Exclusion criteria: carers: non-ambulatory, pregnant or have a medical diagnosis that may be negatively impacted by exercise. Parents whose children have a medical condition or developmental disorder that precludes weight loss using conventional diet and exercise methods

Diagnostic criteria: $\mathrm{BMI} \geq 85$ th percentile

\section{Number of study centres: not reported \\ Treatment before study: none \\ Titration period: none \\ Description of interventions \\ NOURISH parenting intervention}

Based in Social Cognitive Theory (SCT), and emphasises parental role modelling as a primary way children learn health behaviours. Focuses on enhancing parents' self efficacy to make positive changes in eating and exercise behaviours. In addition, cognitive-behavioural strategies such as self monitoring, contingency management and stimulus control incorporated. All sessions involved participatory activities, including self assessments, group discussions and experiential activities. These participatory experiences aimed to enhance overall intervention efficacy

The intervention also informed by Stages of Change Theory and is integrated into the intervention through regular self assessment of barriers to change as well as moderated discussions on the process (pros and cons) of engaging in a healthier lifestyle. Focus on the parents' relationship with everyone in the family, not just the "identified patient" or overweight child, as recommended by Golan and colleagues

Outline of NOURISH session content (each session was 90 minutes):

Session 1 - overview of childhood eating problems and becoming an empowered parent; session 2 - the "toxic environment": how can parents fight back?; Session 3 - nutrition, portion sizes, fruits and vegetables; session 4 - emotional and mindful eating; session 5 parenting styles; session 6 - helping your child develop a healthy relationship with food; session 7 - increasing physical activity; session 8 - reducing physical activity barriers; session 9 - promoting a healthy body image; session 10 - dealing with teasing; session 11 - raising a media-savvy child; session 12 - bringing it all together

All sessions led by doctoral students in psychology working under the supervision of a licensed, clinical psychologist with specific training in group facilitation.

Sessions were video-recorded to allow the investigators to monitor treatment fidelity. Interventionists met weekly with the principal investigator for supervision. These meetings were used to review interventionists' adherence to the treatment protocol, to review programme retention, and to discuss participant interactions

Parents had a 1-hour booster session 2 months after the intervention to allow parents to share with one another their successes, and to elicit suggestions from group leaders and fellow parents regarding barriers they have encountered

Control parenting intervention

Parents in the control group attended a group session moderated by an independent interventionist (a doctoral student in psychology). The session addressed the role of diet and exercise in paediatric overweight. Control participants were mailed publicly available brochures on paediatric overweight on 3 occasions during the study: between weeks 4 
and 5, between weeks 8 and 9, and 2 months after post-testing (the latter of which was meant to match the NOURISH booster session)

Parents and children in both the intervention and control groups received a pedometer. Intervention parents also received a raffle ticket at each session for a USD 75 gift card, which will take place at the final session. Participants who attend the final session were given Certificates of Completion. All parents (i.e. intervention and control groups) were given USD 20 gift cards for completing the pre-test, post-test and the 6-month followup. The study provided childcare for all programme sessions and assessments

Outcomes

Outcomes reported in abstract of publication: child BMI, parents satisfaction, parent behaviour change

$\begin{array}{ll}\text { Study details } & \text { Run-in period: none } \\ \text { Study terminated before regular end (for benefit/because of adverse events): no }\end{array}$

Publication details

Language of publication: English

Non-commercial funding

Publication status: peer-reviewed journal

Stated aim for study

Quote from publication: "The primary aim of this study is to implement and evaluate the feasibility, acceptability, and preliminary effectiveness of NOURISH, a culturally sensitive, parent-only skills-based group intervention, and a single-session, educationonly, control group (parent-only) intervention on overweight children's BMI percentile. The secondary aim of this study is to evaluate the effectiveness of the intervention for improving children's dietary intake, body dissatisfaction, and quality of life. The impact of these two programs on adult participants will also be evaluated, including parental BMI and dietary intake. Parent satisfaction and feedback regarding the NOURISH intervention will also be elicited..."

Notes

Risk of bias

Bias

Authors' judgement

Support for judgement

Random sequence generation (selection Low risk bias)

Quote from publication: "Participants will be randomly assigned (using a random number generator) to one of two parentonly interventions...”

Allocation concealment (selection bias) Unclear risk Comment: no details provided

Blinding of participants and personnel Unclear risk (performance bias)

Subjective outcomes

Blinding of participants and personnel Unclear risk (performance bias)

Objective outcomes
Comment: carers in the control arm were blinded to the aims and hypotheses of this study otherwise no details masking of treat- 
ment assignment

\begin{tabular}{l|l|l}
\hline $\begin{array}{l}\text { Blinding of outcome assessment (detection } \\
\text { bias) } \\
\text { Subjective outcomes }\end{array}$ & Unclear risk & Comment: no details provided \\
\hline
\end{tabular}

Blinding of outcome assessment (detection Unclear risk

Comment: no details provided

bias)

Objective outcome

Incomplete outcome data (attrition bias) Low risk

Comment: no details provided

Subjective outcomes

Incomplete outcome data (attrition bias) Low risk

Objective outcomes

Comment: numbers of drop-outs and withdraws provided and similar across groups. ITT analysis - analysed all the data according to participants' assigned group, whether or not they actually completed the intervention. Used participants' most recent data as post intervention scores

Selective reporting (reporting bias) High risk

Comment: many outcomes were collected but not reported - though it was reported that these were not significantly different from baseline

Other bias

Unclear risk

Comment: although it was made clear why the trial team changed the protocol intervention time from 12 weeks to 6 weeks, this did not seem to help retain participants and meant if there was to be an effect it could not be shown in the health-related quality of life of participants' children

van Grieken 2013

Methods

Cluster randomised controlled trial

Randomisation ratio: $1: 1$

Superiority design:

Participants

Inclusion criteria: child classified as being overweight (not obese) according to international age and gender specific cut-off points for BMI at the well-child visit (country wide health visit in the year a child turns 5 years), attended between September 2007 and October 2008. Parents with at least basic Dutch language skills

Exclusion criteria: obese children with chronic health problems that many influence outcomes

Diagnostic criteria: not reported 
van Grieken 2013 (Continued)

\begin{tabular}{ll}
\hline Interventions & Number of study centres: 9 ( 44 teams) \\
Treatment before study: none \\
Titration period: none \\
Intervention description: \\
Parent-only intervention: motivational interviewing if needed, with information about \\
overweight prevention and healthy lifestyle choices. Initiated at the well-child visit and up \\
to 3 structured counselling sessions could be offered at approximately 3,6 and 12 months \\
later. The session content depended on the stage of behavioural change of the parents \\
(individually tailored). Motivation was assessed by Youth Health Care professionals by \\
creating awareness of the child's weight status and associated consequences. 4 lifestyle- \\
related behaviours could be promoted: playing outdoors, eating breakfast, reducing sweet \\
drinks and sedentary behaviour. Parents choose 1 or 2 behaviours to target during the \\
sessions. Advice was by international guidelines. Information materials provided, diet \\
and activity diaries discussed and family-oriented action plans to change health-related \\
behaviour documented \\
Youth Health care professionals were provided with a half-day training in motivational \\
interviewing techniques, were provided with a workbook with information and practical \\
examples and an information sheet with step-by-step guide to how the information could \\
be applied \\
Control group: parents informed of overweight of their child but usual care (general \\
information about a healthy lifestyle) given
\end{tabular}

Outcomes

Outcomes reported in abstract of publication: BMI, minutes of outside play or TV viewing, having breakfast, number drinks of sweet beverages

Study details

Run-in period: none

Study terminated before regular end (for benefit/because of adverse events): no

Publication details

Language of publication: English

Non-commercial funding

Publication status: peer-review publication

Stated aim for study

Quote from publication: "Children who are overweight (not obese) visiting YHC teams...would have a less increase in BMI and waist circumference at follow-up compared to overweight children visiting YHC teams allocated to the control condition, performing usual care"

Notes

Risk of bias

Bias

Authors' judgement

Support for judgement

Random sequence generation (selection Low risk bias)

Quote from publication: "within each centre, YHC teams were randomised... by means of a computer-generated random number list... random permuted blocks... lengths were 4 or 6 depending on the num- 
van Grieken 2013 (Continued)

ber of teams per Municipal Health Service”

\begin{tabular}{l|l|l}
\hline Allocation concealment (selection bias) & Unclear risk & $\begin{array}{l}\text { Comment: no details of allocation conceal- } \\
\text { ment provided by study authors }\end{array}$ \\
\hline $\begin{array}{l}\text { Blinding of participants and personnel } \\
\text { (performance bias) } \\
\text { Objective outcomes }\end{array}$ & Low risk & $\begin{array}{l}\text { Quote from publication: "parents were not } \\
\text { aware of the research condition they were } \\
\text { allocated to" } \\
\text { Comment: personnel were aware of alloca- } \\
\text { tion. Investigator-assessed outcomes }\end{array}$ \\
\hline
\end{tabular}

Blinding of outcome assessment (detection Low risk bias)

Objective outcome

Incomplete outcome data (attrition bias) Unclear risk

Objective outcomes

Selective reporting (reporting bias)

Low risk

Other bias

Unclear risk
Quote from publication: “... research assistants [measuring weight and height] were blinded to the research condition."

Comment: 2 clusters ( 1 per arm) not analysed. Also states used ITT, numbers analysed for BMI and waist circumference outcomes differ, assume because of ICC for clustering

Comment: all outcomes stated are reported and response from study author concurs

Comment: 211 parent-child dyads in the intervention group did not receive any of the counselling sessions

\section{Small 2013}

Methods

Randomisation ratio: not reported
Superiority design

Participants
Inclusion criteria: parents of overweight or obese 4- to 8-year-old

Exclusion criteria: children who had uncontrolled medical problems (e.g. asthma) that might preclude them from fully participating in the intervention

Diagnostic criteria: described only as overweight or obese

Number of study centres: unclear

Treatment before study: none

Titration period: none

Description of interventions

Before each of the sessions for either group, parents were provided with age-appropriate, audio-taped, educational information on a range of topics. Each group had 4 face-toface sessions held for 30-60 minutes and 3 telephone calls between sessions (duration unclear)

Parent intervention: 
Theoretically based intervention. Parents were offered educational information about the establishment of healthy habits in young children, nutritional information, information regarding increasing physical activity and decreasing sedentary time, and age-specific information regarding the child's behaviour in response to change

4 face-to-face sessions during which trained research assistants used principles of brief motivational interviewing (i.e. elicit information from the participant, provide nonjudgemental information, and elicit the participant's understanding; to collaborate with parents on identifying specific realistic healthy lifestyle goals, developing clear steps to reach those goals, routinely having the parents re-evaluate progress, and identifying new goals as needed)

Parents were provided with specific feedback about their child (i.e. physical activity and dietary intake) before establishing goals for their child and family. All face-to-face parent intervention sessions were separated by $4-6$ weeks to provide each family with time to enact planned changes, encounter child responses to those changes and review new educational information before the next face-to-face session

Telephone calls were made to each parent to:

- review the established goals and planned steps,

- check with the parent regarding progress toward planned changes,

- answer questions,

- encourage review of newly provided educational materials

- establish a date and time for the next face-to-face appointment.

At each of the 4 measurement time points, parents were offered USD 35 as remuneration for their time in completing the various measurements. Each child was given a group specific (e.g. treatment group and control group) bag of toys to facilitate activities that parents would be encouraged to complete with their child

Sessions taken by trained research assistants who were also supervised

Control:

Parents were provided with educational age-appropriate, evidence-based health and safety information (e.g. care for thermal injuries, first-aid care, and care for insect bites and stings) that is specific to parenting in the southwest US. Parents met with a control interventionist and in a similar way were encouraged to make health and safety goals for their family (e.g. development of first-aid materials and identification of a fire escape plan)

Telephone calls were made to each parent as described above

Outcomes

Outcomes reported in abstract of publication: waist circumference, waist-by-height ratio, $\mathrm{BMI}$ and $\mathrm{BMI}$ percentile

Study details

Run-in period: none

Study terminated before regular end (for benefit/because of adverse events): no

Publication details

Language of publication: English

Non-commercial funding

Publication status: peer-reviewed journal

Stated aim for study

Quote from publication: "The purpose of this randomised control pilot study was to determine the feasibility and preliminary effects of a theoretically based, primary care intervention on the physical outcomes of 60 overweight/obese preschool/early schoolaged 4- to 8-year-old children..."

Parent-only interventions for childhood overweight or obesity in children aged 5 to II years (Review) 
Small 2013 (Continued)

Notes

Risk of bias

\begin{tabular}{|c|c|c|}
\hline Bias & Authors' judgement & Support for judgement \\
\hline $\begin{array}{l}\text { Random sequence generation (selection } \\
\text { bias) }\end{array}$ & Unclear risk & $\begin{array}{l}\text { Quote from publication: "each parent- } \\
\text { child dyad was randomly assigned to the } \\
\text { treatment or control condition..." } \\
\text { Comment: no description provided }\end{array}$ \\
\hline Allocation concealment (selection bias) & Unclear risk & Comment: no description provided \\
\hline $\begin{array}{l}\text { Blinding of participants and personnel } \\
\text { (performance bias) } \\
\text { Objective outcomes }\end{array}$ & Unclear risk & Comment: no details provided \\
\hline $\begin{array}{l}\text { Blinding of outcome assessment (detection } \\
\text { bias) } \\
\text { Objective outcome }\end{array}$ & Unclear risk & Comment: no details provided \\
\hline $\begin{array}{l}\text { Incomplete outcome data (attrition bias) } \\
\text { Objective outcomes }\end{array}$ & Low risk & $\begin{array}{l}\text { Quote from publication: "Seven parent- } \\
\text { child dyads returned incomplete T1 data, } \\
\text { and thus we removed data from those dyads } \\
\text { from all other analyses. As a result, experi- } \\
\text { mental and control group sample sizes were } \\
33 \text { and } 27 \text {, respectively" } \\
\text { Comment: multiple imputation tech- } \\
\text { niques to account for the missing data in } \\
\text { subsequent analyses for the remaining } 60 \\
\text { parent-child dyads }\end{array}$ \\
\hline Selective reporting (reporting bias) & Unclear risk & $\begin{array}{l}\text { Comment: all outcomes reported as stated } \\
\text { but not enough information to judge }\end{array}$ \\
\hline Other bias & Unclear risk & Comment: not enough detail to judge \\
\hline
\end{tabular}

Esfarjani 2013

\begin{tabular}{l|l} 
Methods & Parallel randomised controlled trial \\
& Randomisation ratio: $1: 1$ \\
& Superiority design \\
\hline
\end{tabular}

Participants

Inclusion criteria: aged 7 years, $\geq 95$ th percentile of BMI for age

Exclusion criteria: mental retardation, psychiatric symptoms, current obesity treatment, chronic disease and use of medication

Diagnostic criteria: obesity defined as BMI 95th percentile for age by 2000 reference 
standards

$\begin{array}{ll}\text { Interventions } & \text { Number of study centres: } 1 \\ \text { Treatment before study: none } & \text { Titration period: none } \\ \text { Intervention description: } \\ \text { Parent-only: } \\ 12 \text { session training programme over } 6 \text { months } \\ 8 \text { weekly sessions for the first } 2 \text { months, then } 4 \text { monthly sessions. Each session lasts } 4 \\ \text { hours, including a review of parent progress in implementing strategies developed for } \\ \text { changing child's eating or exercise habits, and the specific topic of the day, such as learning } \\ \text { about the reasons of the childhood obesity, receiving nutritional information (e.g. food } \\ \text { pyramid, food choices, food labels, food preparation and cooking, eating habits, regular } \\ \text { meals, controlling environments that stimulate overeating, special dietary consideration } \\ \text { during holidays and at the restaurants) and guidelines for physical activity and reducing } \\ \text { sedentary behaviours (e.g. reduce watching TV and playing computer games, use stairs } \\ \text { instead of lifts and play outside instead of inside) } \\ \text { Control: } \\ 2 \text { sessions of training programme (occurred after intervention group's 6-month training } \\ \text { programme), no details provided }\end{array}$

Outcomes

Outcomes reported in abstract of publication: weight, waist and hip circumference, cholesterol, serum triglycerides, food group consumption, TV and computer time, walking time

\begin{tabular}{ll} 
Study details & $\begin{array}{l}\text { Run-in period: none } \\
\text { Study terminated before regular end }\end{array}$ \\
\hline Publication details & $\begin{array}{l}\text { Language of publication: English } \\
\text { Non-commercial funding } \\
\text { Peer review publication }\end{array}$ \\
\hline
\end{tabular}

Stated aim for study

Quote from publication: "to assess the effect of lifestyle modification family-based intervention in young Iranian children"

Notes

Risk of bias

\begin{tabular}{|c|c|c|}
\hline Bias & Authors' judgement & Support for judgement \\
\hline $\begin{array}{l}\text { Random sequence generation (selection } \\
\text { bias) }\end{array}$ & Unclear risk & $\begin{array}{l}\text { Quote from publication: "children got a } \\
\text { code number and were randomly divided } \\
\text { into two groups" } \\
\text { Comment: randomly assigned }\end{array}$ \\
\hline Allocation concealment (selection bias) & Unclear risk & $\begin{array}{l}\text { Comment: not enough information to } \\
\text { judge }\end{array}$ \\
\hline
\end{tabular}

Parent-only interventions for childhood overweight or obesity in children aged 5 to II years (Review) 


\section{Esfarjani 2013 (Continued)}

Blinding of participants and personnel Unclear risk (performance bias)

Objective outcomes
Comment: adjudicated/investigatorassessed, no other details

Blinding of outcome assessment (detection Unclear risk bias)

Objective outcome

Incomplete outcome data (attrition bias) High risk Objective outcomes
Comment: no details provided
Selective reporting (reporting bias) Unclear risk
Comment: numbers only reported, numbers in flow chart and results do not match, differential drop-out rates

Comment: all outcomes reported as stated but not enough information to judge

Comment: not enough information to judge

Moens 2012

Methods

Parallel randomised controlled trial

Randomisation ratio: not reported

Superiority design

Participants

Inclusion criteria: child aged 6-12 years, $20-85 \%$ overweight, medical clearance form a physician

Exclusion criteria: secondary overweight caused by endocrinological, chromosomal or hypothalamic disease or mental retardation

Diagnostic criteria: as above

Interventions

\section{Number of study centres: 1 \\ Treatment before study: none \\ Titration period: none \\ Intervention description:}

Parent-only intervention:

6 group meetings of 2 hours each over a 5-month period. Provided information with a focus on weight control not weight loss to re-establish a sense of healthy balance between energy intake and energy expenditure. Parent workbook. Education on different food groups, detailed product information and child-friendly recipes. Used the Food Pyramid. Parenting skills focused on understanding of eating habits and lifestyles, cognitive and behavioural barriers to change, general parenting skills of positive involvement, monitoring, problem-solving skills and maintaining positive changes already effected; self control and healthier lifestyle sessions (full details of content provided)

Sessions conducted by dietician and a psychologist under supervision of a behavioural therapist and a manual for each session was available

Control:

Waiting list control 
Moens 2012 (Continued)

\section{Risk of bias}

\section{Bias}

Random sequence generation (selection High risk bias)

\section{Authors' judgement}

\begin{tabular}{l|l}
\hline Outcomes & $\begin{array}{l}\text { Outcomes reported in abstract of publication: BMI, parental report of child's eating } \\
\text { behaviour, familial health principles }\end{array}$ \\
\hline Study details & $\begin{array}{l}\text { Run-in period: none } \\
\text { Study terminated before regular end (for benefit/because of adverse events): no }\end{array}$ \\
\hline Publication details & $\begin{array}{l}\text { Language of publication: English } \\
\text { Commercial funding } \\
\text { Peer review publication }\end{array}$ \\
\hline Stated aim for study & $\begin{array}{l}\text { Quote from publication “To evaluate a parent-led intervention for overweight children } \\
\text { between } 6 \text { and } 12 \text { years old" }\end{array}$ \\
\hline
\end{tabular}

Notes

Also report a follow-up study comparing all families post intervention with a sample of

families who did not respond to the original invitation. Not relevant here 
Moens 2012 (Continued)

Objective outcome

\begin{tabular}{|c|c|c|}
\hline $\begin{array}{l}\text { Incomplete outcome data (attrition bias) } \\
\text { Subjective outcomes }\end{array}$ & Unclear risk & $\begin{array}{l}\text { Comment: questionnaire completion rates } \\
\text { provided and some withdrawn by study au- } \\
\text { thors as missing items }\end{array}$ \\
\hline $\begin{array}{l}\text { Incomplete outcome data (attrition bias) } \\
\text { Objective outcomes }\end{array}$ & Unclear risk & $\begin{array}{l}\text { Comment: reports numbers but not rea- } \\
\text { sons for drop-out. All drop-outs from wait- } \\
\text { ing list control group so some imbalance. } \\
\text { No description of how analysed }\end{array}$ \\
\hline Selective reporting (reporting bias) & High risk & $\begin{array}{l}\text { Comment: subjective outcomes not re- } \\
\text { ported at baseline or follow-up for the } 2 \\
\text { groups separately but are reported for both } \\
\text { combined }\end{array}$ \\
\hline Other bias & Unclear risk & $\begin{array}{l}\text { Comment: is reported to be a pilot study } \\
\text { so unclear if sample size is appropriate to } \\
\text { detect a difference }\end{array}$ \\
\hline
\end{tabular}

Raynor 2012a

Methods

Participants

\section{Parallel randomised controlled trial \\ Randomisation ratio: $1: 1: 1$ \\ Superiority design}

Inclusion criteria: $\geq 85$ th percentile for BMI as determined by the Centers for Disease Control growth charts, having no dietary or physical activity restrictions

Exclusion criteria: participating parent could not read English, had a psychological disorder that would impair ability to participate or if the family was planning to move out of the area during the programme

Diagnostic criteria: $\mathrm{BMI} \geq 85$ th percentile
Number of study centres: not reported

Treatment before study: none

Titration period: none

Description of interventions

Behavioural parent-only intervention:

8 sessions, 45 minutes each

Focused on increasing child growth monitoring and providing feedback to families

2 interventions that combined the parent-only intervention with a 6-month, behavioural, parent-only intervention that focused on 2 energy-balance (diet) behaviours:

[DECREASE]

Decreasing sugar sweetened beverage and sweet and salty snack food intake. Children and parents reduced intake of sweet and salty snack foods (i.e. candy, cookies, ice cream, chips, nuts) to $\leq 3$ servings/week, and sugar sweetened beverages (i.e. soda, Kool-aid, sweetened tea, non-100\% fruit juice, sports drinks) to $\leq 3$ servings/week [INCREASE] 
Increasing fruit, vegetable and low-fat dairy intake. Children and parents were encouraged to consume 2 servings/day of whole fruit, 3 servings/day of vegetables and 2 servings/day of low-fat dairy products

Sessions covered behavioural lessons and emphasised monitoring of targeted behaviours, pre-planning, problem solving, shaping, setting goals, positive reinforcement, stimulus control and parental modelling of targeted behaviours. These behavioural strategies are endorsed in both the 1997 and 2007 recommendations. Children and their parents self monitored the targeted behaviours and turned in records at each meeting

Families received USD 20 for completing each of the 6- and 12-month assessments

Based on behavioural economics theory (changing a substitute behaviour of a target behaviour enhances the feeling of choice for engaging in and liking the targeted behaviour, which could increase long-term adherence). Meetings were led by an experienced research-staff therapist (either master or doctoral level) with expertise in nutrition or exercise science, and behaviour modification

Following the 6-month intervention, all families received feedback on growth at 9 months, and final assessments were conducted at 12 months

Publication details

Language of publication: English

Non-commercial funding

Publication status: peer-reviewed journal treatment recommendations, within two randomised trials"

\begin{tabular}{|c|c|c|}
\hline Bias & Authors' judgement & Support for judgement \\
\hline $\begin{array}{l}\text { Random sequence generation (selection } \\
\text { bias) }\end{array}$ & Low risk & $\begin{array}{l}\text { Quote from publication: "Participants in } \\
\text { each trial were randomly assigned to one of } \\
\text { three interventions in a 1:1:1 allocation ra- } \\
\text { tio. Using random permuted blocks within } \\
\text { strata (gender), cards with intervention as- } \\
\text { signment were sealed in an envelope by re- } \\
\text { search staff not engaged in intervention or } \\
\text { assessments and provided to families at a } \\
\text { randomisation visit, following completion } \\
\text { of baseline assessments" }\end{array}$ \\
\hline
\end{tabular}


Raynor 2012a (Continued)

\begin{tabular}{|c|c|c|}
\hline Allocation concealment (selection bias) & Low risk & $\begin{array}{l}\text { Quote from publication: "Participants in } \\
\text { each trial were randomly assigned to one of } \\
\text { three interventions in a 1:1:1 allocation ra- } \\
\text { tio. Using random permuted blocks within } \\
\text { strata (gender), cards with intervention as- } \\
\text { signment were sealed in an envelope by re- } \\
\text { search staff not engaged in intervention or } \\
\text { assessments and provided to families at a } \\
\text { randomisation visit, following completion } \\
\text { of baseline assessments" }\end{array}$ \\
\hline
\end{tabular}

Blinding of participants and personnel Unclear risk (performance bias)

Objective outcomes

Comment: masking of carers and participants to allocation but unclear if blinded to intervention

Blinding of outcome assessment (detection Low risk bias)

Objective outcome

Quote from publication: "Dependent measures were collected by trained researchstaff blinded to treatment assignment"

Comment: outcome assessors masked to treatment assignment

Incomplete outcome data (attrition bias) Low risk Objective outcomes

Comment: ITT analysis. Missing data filled using a multiple imputation strategy. Specifically, for each participant with a missing BMI z score value, 5 random variables from a normal distribution that has a mean equal to the baseline BMI $\mathrm{z}$ score and variance equal to the estimated variance for BMI $\mathrm{z}$ score of other participants at the time where BMI $\mathrm{z}$ score is missing

Selective reporting (reporting bias) Unclear risk

Comment: all outcomes reported as stated but not enough information to judge

Other bias

Unclear risk

Comment: not enough detail to judge

Raynor 2012b

Methods

Parallel randomised controlled trial

Randomisation ratio: $1: 1: 1$

Superiority design

Participants

Inclusion criteria: $\geq 85$ th percentile for BMI as determined by the Centers for Disease Control growth charts, having no dietary or physical activity restrictions

Exclusion criteria: participating parent could not read English, had a psychological disorder that would impair ability to participate or if the family was planning to move out of the area during the programme

Diagnostic criteria: $\mathrm{BMI} \geq 85$ th percentile 
Raynor 2012b (Continued)

Number of study centres: not reported
Treatment before study: none
Titration period: none
Description of interventions
Behavioural parent-only intervention:
as above.
2 additional interventions were:
[TRADITIONAL]

Decreasing sugar-sweetened beverage intake and increasing physical activity. Encouraged children to reach 60 minutes/day (parents 30 minutes/day) of moderate-intensity physical activity most days of the week and for children and parents to consume $\leq 3$ servings of sugar-sweetened beverages/week.

[SUBSTITUTE]

Increasing low-fat milk intake and decreasing TV watching, encouraged children and parents to watch $\leq 2$ hours of TV/day and to consume 2 servings of low-fat milk/day

Outcomes

Outcomes reported in abstract of publication: BMI z score, energy intake

Study details

Run-in period: none

Study terminated before regular end (for benefit/because of adverse events): no

Publication details

Language of publication: English

Non-commercial funding

Publication status: peer-reviewed journal

Stated aim for study

Quote from publication: "to examine the efficacy of U.S. primary care paediatric obesity treatment recommendations, within two randomised trials"

Notes

There are 2 comparisons of relevance to this review: the parent-only vs. the substitute group and the parent-only vs. the increase group

Risk of bias

Bias

Authors' judgement

Support for judgement

Random sequence generation (selection Low risk bias)

Quote from publication: "Participants in each trial were randomly assigned to one of three interventions in a 1:1:1 allocation ratio. Using random permuted blocks within strata (gender), cards with intervention assignment were sealed in an envelope by research staff not engaged in intervention or assessments and provided to families at a randomisation visit, following completion of baseline assessments" 
Raynor 2012b (Continued)

\begin{tabular}{|c|c|c|}
\hline Allocation concealment (selection bias) & Low risk & $\begin{array}{l}\text { Quote from publication: "Participants in } \\
\text { each trial were randomly assigned to one of } \\
\text { three interventions in a 1:1:1 allocation ra- } \\
\text { tio. Using random permuted blocks within } \\
\text { strata (gender), cards with intervention as- } \\
\text { signment were sealed in an envelope by re- } \\
\text { search staff not engaged in intervention or } \\
\text { assessments and provided to families at a } \\
\text { randomisation visit, following completion } \\
\text { of baseline assessments..." }\end{array}$ \\
\hline
\end{tabular}

Blinding of participants and personnel Unclear risk (performance bias)

Objective outcomes

Comment: masking of carers and participants to allocation but unclear if blinded to intervention

Blinding of outcome assessment (detection Low risk bias)

Objective outcome

Quote from publication: "Dependent measures were collected by trained researchstaff blinded to treatment assignment.."

Comment: outcome assessors masked to treatment assignment

Incomplete outcome data (attrition bias) Low risk Objective outcomes

Comment: ITT analysis. Missing data filled using a multiple imputation strategy. Specifically, for each participant with a missing BMI z score value, 5 random variables from a normal distribution that has a mean equal to the baseline BMI $\mathrm{z}$ score and variance equal to the estimated variance for BMI $\mathrm{z}$ score of other participants at the time where BMI $\mathrm{z}$ score is missing

Selective reporting (reporting bias) Unclear risk

Comment: all outcomes reported as stated but not enough information to judge

Other bias

Unclear risk

Comment: not enough detail to judge

\section{Magarey 2011}

$\begin{array}{ll}\text { Methods } & \text { Parallel randomised controlled trial } \\ \text { Randomisation ratio: not reported } \\ \text { Superiority design }\end{array}$

Participants

Inclusion criteria: aged 5.0-9.9 years, overweight (International Obesity Task Force definition) and prepubertal (Tanner stage 1), having a carer willing to attend sessions and able to speak English

Exclusion criteria: BMI z score of 4.0, having a syndromal cause of obesity, using medications that influence weight, having a physical or developmental disability, having a chronic illness or having a sibling enrolled in the study 


\begin{tabular}{|c|c|}
\hline Interventions & $\begin{array}{l}\text { Number of study centres: } 3 \\
\text { Treatment before study: none } \\
\text { Titration period: none } \\
\text { Description of interventions } \\
24 \text {-week intervention delivered by a dietician (accredited training for the parenting skills } \\
\text { component) } \\
2 \text { groups: parent healthy lifestyle group and healthy lifestyle group } \\
\text { The interventions included } 12 \text { (parent group) or } 8 \text { (healthy lifestyle) } 90 \text { - to } 120 \text {-minute } \\
\text { group sessions (open to both parents but mostly attended by mothers) and } 4 \text { tele- } \\
\text { phone sessions, delivered over } 6 \text { months with tapered frequency (weekly, bimonthly, } \\
\text { then monthly). Details of the standardised parenting skills programme and the healthy } \\
\text { lifestyle education sessions reported (references given). The } 4 \text { telephone sessions alter- } \\
\text { nated with the last } 4 \text { group sessions for both arms, using a standard protocol } \\
\text { Parenting healthy lifestyle: } \\
\text { The Positive Parenting Program (Triple P) was delivered in } 4 \text { sessions before the healthy } \\
\text { lifestyle component. It is a standardised and evaluated generic parenting programme } \\
\text { widely used in Australia and provides comprehensive facilitator training } \\
\text { Healthy lifestyle: } \\
\text { The } 8 \text { sessions included recommendations on specific core food servings; practical skills } \\
\text { for healthy eating, reduced sedentary behaviours and increased activity; and monitoring } \\
\text { of lifestyle behaviours and roles and responsibilities around eating, managing appetite, self } \\
\text { esteem and teasing. Children and siblings participated in fun, non-competitive activity } \\
\text { sessions run by physical activity educators. These sessions provided optional active child } \\
\text { care for participants in both groups and were not part of the intervention }\end{array}$ \\
\hline
\end{tabular}

Publication details

Language of publication: English Non-commercial funding

Publication status: peer-reviewed journal

Quote from publication: “... to evaluate (1) the effectiveness of a healthy lifestyle intervention for overweight children aged 5 to 9 years that targets parents as the agents of change and (2) whether additional specific parenting skills training would improve parenting skills and enhance the intervention effect. The long-term effect ( 2 years from baseline) and the immediate postintervention effect (at completion of the intervention, 6 months from baseline) were assessed. We also aimed to confirm gender differences reported in our previous study...”

Notes

\section{Risk of bias}




\section{Magarey 2011 (Continued)}

\begin{tabular}{l|l|l|}
$\begin{array}{l}\text { Random sequence generation (selection } \\
\text { bias) }\end{array}$ & Low risk & $\begin{array}{l}\text { Quote from publication: “.. After baseline } \\
\text { measurements, participants were allocated }\end{array}$ \\
& to intervention groups using computer- \\
& generated randomisation schedules strati- \\
& fied according to gender and recruitment \\
site and prepared by staff not otherwise in- \\
volved in the study"
\end{tabular}

Allocation concealment (selection bias) Low risk

Quote from publication: "Allocation was concealed in opaque, sequentially numbered, sealed envelopes and opened by parents after completion of baseline measurements..."

Blinding of participants and personnel High risk (performance bias)

Comment: a single-blinded randomised Subjective outcomes controlled trial blind at point of allocation, but not blinded to which intervention they received

Blinding of participants and personnel High risk (performance bias)

Comment: a single-blinded randomised controlled trial blind at point of allocation,

Objective outcomes but not blinded to which intervention they received

Blinding of outcome assessment (detection Low risk bias)

Comment: those who took measurements were blinded to type of intervention

Subjective outcomes

Blinding of outcome assessment (detection Low risk bias)

Objective outcome
Comment: those who took measurements were blinded to type of intervention
Incomplete outcome data (attrition bias) High risk

Subjective outcomes
Comment: stated ITT was conducted using all available data according to allocation, regardless of attendance (details provided). However, a second per-protocol analysis was performed that included only those who attended $\geq 75 \%$ of the programme sessions. The potential effect of missing data were explored using $\mathrm{T}$ tests to compare the baseline and 6-month BMI $\mathrm{z}$ scores of those who remained and those who were lost to follow-up. No reasons for drop-outs were given. It is unclear whether data presented were ITT or not

Comment: stated ITT was conducted using all available data according to allocation, regardless of attendance (details pro- 
vided). However, a second per-protocol analysis was performed that included only those who attended $\geq 75 \%$ of the programme sessions. The potential effect of missing data were explored using $\mathrm{T}$ tests to compare the baseline and 6-month BMI $\mathrm{z}$ scores of those who remained and those who were lost to follow-up. No reasons for drop-outs were given. It is unclear whether data presented were ITT or not

Selective reporting (reporting bias) High risk

Unclear risk
Comment: states health-related quality of life measured but no data reported

Comment: no true control group. Parenting outcomes only given for the whole sample, not split into intervention and control groups - therefore success of intervention in each group cannot be compared. Retention rates moderate for the long follow-up period. Unclear if ITT analysis was performed. Likely the participants who dropped out the study were more overweight

Jansen 2011

Methods

Parallel randomised controlled trial

Randomisation ratio: not reported

Superiority design

Participants

Inclusion criteria: parents participated in the treatment voluntarily. Children's percentage of overweight had to be at least $130 \%$

Exclusion criteria: none stated

Diagnostic criteria: overweight (as above)

Interventions

Number of study centres: 3

Treatment before study: none

Titration period: none

Description of interventions:

Parental CBT:

'Finger in the pie' was a cognitive behavioural treatment and each session addressed a different theme associated with childhood overweight. The purpose was not purely to present information, but to teach parents to think of alternatives and possible solutions themselves. This way, future coping abilities were addressed. The following themes were included: creating realistic expectations concerning the development of their children's weight status, modifying eating and exercising habits, knowledge on how parents can influence the behaviour of their children (e.g. by modelling and by the use of control

Parent-only interventions for childhood overweight or obesity in children aged 5 to I I years (Review) 
Jansen 2011 (Continued)

and rewards), information on the development of overweight, handling feelings of guilt, and recognising and handling a child with low self esteem. So, instead of purely focusing on nutrition and physical activity, a substantial part of the treatment was devoted to enhancing parenting tactics (e.g. teaching parents to ignore undesirable behaviours and to reward desirable behaviours). This aspect of the treatment combined with extensively discussing parental control makes the current intervention distinguishing

Of the 2-hour sessions, the first hour was interactive. 1 of the main goals of this first hour was to identify wrong thought patterns and challenging these patterns. The second hour of each session was more informative and practical by nature

Behavioural and nutritional components

Session 1 - the part that parents play; session 2 - eating behaviour; session 3 - physical exercise; session 4 - parental control; session 5 - be in good spirit; session 6 - food and party; session 7 - relapse; session 8 - responsibility

Intervention carried out according to a protocol, written by the first and the second authors and carried out by trained cognitive behavioural therapists

Waiting list control:

Offered the treatment after 6 months

Outcomes

Outcomes reported in abstract of publication: BMI percentile, relapse, psychopathology, self esteem and negative thoughts

Study details

Run-in period: none

Study terminated before regular end (for benefit/because of adverse events): no

Publication details

Language of publication: English

Funding not stated

Publication status: peer-reviewed journal

Stated aim for study

Quote from publication: "The aim of the current study is to investigate whether a treatment that aims at parents exclusively (by targeting eating and exercise behaviours, and supporting self-esteem of the child in a cognitive-behavioural manner) would be successful in reducing their children's overweight. Such a treatment might lead to better results than treatments focusing on children, as parents play an important role in their children's eating and exercising behaviours, and in promoting their self-esteem..."

Notes

Randomisation was broken because 9 participant families from the waiting list control were included in the intervention arm, the study did not report the numbers randomised to each group, just the total numbers randomised, the group sizes were unbalanced (59 vs. 39), and the paper states that 9 were 'included in the treatment group'

Risk of bias

Bias

Authors' judgement

Support for judgement

Random sequence generation (selection Unclear risk bias)

Quote from publication: "Parents were randomly assigned to either the treatment group or the waiting-list control group" Comment: no description of randomisation process

Parent-only interventions for childhood overweight or obesity in children aged 5 to II years (Review)

Copyright $\Subset 2015$ The Cochrane Collaboration. Published by John Wiley \& Sons, Ltd. 
Jansen 2011 (Continued)

\begin{tabular}{|c|c|c|}
\hline Allocation concealment (selection bias) & Unclear risk & Comment: no details provided \\
\hline $\begin{array}{l}\text { Blinding of participants and personnel } \\
\text { (performance bias) } \\
\text { Subjective outcomes }\end{array}$ & Unclear risk & Comment: no details provided \\
\hline $\begin{array}{l}\text { Blinding of participants and personnel } \\
\text { (performance bias) } \\
\text { Objective outcomes }\end{array}$ & Unclear risk & Comment: no details provided \\
\hline $\begin{array}{l}\text { Blinding of outcome assessment (detection } \\
\text { bias) } \\
\text { Subjective outcomes }\end{array}$ & Unclear risk & Comment: no details provided \\
\hline $\begin{array}{l}\text { Blinding of outcome assessment (detection } \\
\text { bias) } \\
\text { Objective outcome }\end{array}$ & Unclear risk & Comment: no details provided \\
\hline $\begin{array}{l}\text { Incomplete outcome data (attrition bias) } \\
\text { Subjective outcomes }\end{array}$ & Low risk & Comment: no details provided \\
\hline $\begin{array}{l}\text { Incomplete outcome data (attrition bias) } \\
\text { Objective outcomes }\end{array}$ & Low risk & $\begin{array}{l}\text { Comment: ITT analysis. The missing val- } \\
\text { ues at the follow-up meeting were re- } \\
\text { placed by the last-observation-carried-for- } \\
\text { ward. The missing in-between values (post- } \\
\text { treatment) were replaced by the mean of } \\
\text { the values before treatment and at 3-month } \\
\text { follow-up. Numbers of drop-outs reported }\end{array}$ \\
\hline Selective reporting (reporting bias) & Unclear risk & $\begin{array}{l}\text { Comment: all outcomes reported as stated } \\
\text { but not enough information to judge }\end{array}$ \\
\hline Other bias & High risk & $\begin{array}{l}\text { Comment: as only } 9 \text { of the } 48 \text { families in } \\
\text { the waiting-list control group eventually } \\
\text { decided to participate in the treatment af- } \\
\text { ter the waiting period, it was decided to } \\
\text { include these } 9 \text { families in the treatment } \\
\text { group, and to disregard their data from the } \\
\text { waiting period. These } 9 \text { families did not } \\
\text { differ from the original treatment group or } \\
\text { the control group }\end{array}$ \\
\hline
\end{tabular}


Methods

Participants
Parallel randomised controlled trial

Randomisation ratio: not reported

Superiority design

Inclusion criteria: overweight or obese (defined according to age- and sex-specific international BMI cut-off points (reference provided); aged 5-9 years; pre-pubertal (no pubic hair - Tanner stage 1); generally healthy

Exclusion criteria: extreme obesity (BMI z score > 4); known syndromal cause of obesity; long-term steroid use; medications associated with weight gain; chronic illness; significant dietary restrictions

Diagnostic criteria: overweight or obese (defined according to age- and sex-specific international BMI cut-off points

Interventions

Number of study centres: not reported

Treatment before study: not reported

Titration period: not reported

Detailed description of all interventions

Parent DIET Intervention:

Aimed at parents, not children. Informs parents of how to improve their child's diet. Based on the "Health Belief Model" (reference provided).

10 parent focused face-to-face group sessions - each 2 hours long. 3 monthly telephone calls for the first 3 months of the intervention. Goal setting, problem-solving, rolemodelling and positive reinforcement are used to manage changes in food behaviours and strategies incorporated to help parents increase their confidence in making changes related to their goals. The structure and content of the programme uses a cognitive behavioural, solution-focused approach from the emerging field of health coaching. Behavioural change targeted during the sessions to reduce total energy and fat intakes, increase fruit and vegetable intake and make healthy beverage and snack choices. Practical advice about food shopping and preparation is provided with the sessions including a didactic component, group work and practical activities

Delivered by a dietician

Parent and child physical activity (child was main focus):

Parent encouraged to set realistic short- to medium-term SMART goals for increasing physical activity and reducing sedentary behaviours. Asked to identify barriers in their family lives that may prevent their child from participating in sufficient physical activity or that leads to their child spending excessive amounts of time in small screen recreation Children attended $10 \times 2$-hour face-to-face weekly sessions. Each week children participate in a variety of activities aimed at improving their mastery of 12 fundamental movement skills (run, jump, leap, hop, slide, gallop, strike, roll, kick, throw, catch, bounce) . Each session covered 3 fundamental movement skills, such that over the course of the 10 weeks each skill is re-visited, although the focus is on more complex components of the skill, in subsequent sessions. Skill mastery is aided by adherence to lesson plans for each skill incorporating several learning stages:

- contextual stage (questions children as to what games, sports and activities require mastery of the specific skill and how the skill is performed proficiently);

- exploration stage (allows children to explore the different movement patterns related to the skill using movement concepts such as force, speed, levels and relationships);

- guided discovery stage (isolates specific components of a skill and using a problem 
Collins 2011 (Continued)

solving approach, guides children to discover the correct way to perform the skill);

- skill application stage (applies the skills in small drill activities and modified game contexts)."

1 Refresher session (2 hours) attended at week 18; 3 monthly telephone calls at weeks 14,18 and 22 of the invention

To maximise the children's competence and confidence, they were strongly encouraged to practice the fundamental movement skills at home with their parents or siblings (or both) , between each group session. Each participant given a 'Home-challenge folder', which included fun, relevant and developmentally appropriate activities enabling practice of skills at home. The home challenges took approximately 30 minutes and children were encouraged to complete 3 sessions each week

Based on the "Competence Motivation Theory" (reference provided). Delivered by physical education teachers

Parent-Child Physical Activity + DIET:

Same components as the DIET and physical activity interventions. 25 sessions. Delivered by physical education teachers and dieticians.

10 Parent-focused face-to-face group sessions - each were 2 hours long (10 weeks); 10 child-focused face-to-face group sessions - each 2 hours long (10 weeks); 1 refresher session ( 2 hours) attended at week 18; 3 monthly telephone calls at weeks 14, 18 and 22 of the invention; 1 parent workshop - 1 hour long

Outcomes

Outcomes reported in abstract of publication: BMI z score, waist measurements, metabolic outcomes

Study details

Run-in period: not reported

Study terminated before regular end (for benefit/because of adverse events): no

Publication details

Language of publication: English

Non-commercial funding

Publication status: peer-reviewed journal

Stated aim for study

Quote from publication: "Outcomes of childhood obesity interventions are rarely reported beyond 1 year. We hypothesized that the impact on the BMI z score from a childcentred physical-activity program in combination with a parent-entered dietary-modification program would be greater than either program conducted alone at 24 months' after baseline"

Notes

There were 2 comparisons of relevance to this review: the parent-only vs. parent-child physical activity group and the parent-only vs. the parent-child physical activity and diet group

\section{Risk of bias}

\section{Bias}

Random sequence generation (selection Low risk bias)
Authors' judgement

\section{Support for judgement}

Quote from publication: “To randomly allocate participants to one of the three intervention groups the bias coin method of allocation, using a computer-based random 


\begin{tabular}{|c|c|c|}
\hline & & $\begin{array}{l}\text { number-producing algorithm, is used. This } \\
\text { method ensures an equal chance of alloca- } \\
\text { tion to each group. Stratification by gender } \\
\text { and site is done to ensure an equal repre- } \\
\text { sentation in groups at each site. Only one } \\
\text { study member at each site has access to the } \\
\text { allocation codes and these are stored on a } \\
\text { password-protected computer" }\end{array}$ \\
\hline Allocation concealment (selection bias) & Unclear risk & Comment: no details provided \\
\hline $\begin{array}{l}\text { Blinding of participants and personnel } \\
\text { (performance bias) } \\
\text { Subjective outcomes }\end{array}$ & High risk & Comment: no blinding \\
\hline $\begin{array}{l}\text { Blinding of participants and personnel } \\
\text { (performance bias) } \\
\text { Objective outcomes }\end{array}$ & High risk & Comment: no blinding \\
\hline $\begin{array}{l}\text { Blinding of outcome assessment (detection } \\
\text { bias) } \\
\text { Subjective outcomes }\end{array}$ & Low risk & $\begin{array}{l}\text { Quote from publication: "Outcome mea- } \\
\text { sures were assessed at baseline and } 6,12 \text {, } \\
\text { and } 24 \text { months by trained assessors blinded } \\
\text { to group assignment..." }\end{array}$ \\
\hline $\begin{array}{l}\text { Blinding of outcome assessment (detection } \\
\text { bias) } \\
\text { Objective outcome }\end{array}$ & Low risk & $\begin{array}{l}\text { Comment: "Outcome measures were as- } \\
\text { sessed at baseline and } 6,12 \text {, and } 24 \text { months } \\
\text { by trained assessors blinded to group as- } \\
\text { signment..." }\end{array}$ \\
\hline $\begin{array}{l}\text { Incomplete outcome data (attrition bias) } \\
\text { Subjective outcomes }\end{array}$ & High risk & $\begin{array}{l}\text { Comment: ITT performed, however, high } \\
\text { numbers not completing ( } 35 \% \text { to } 51 \% \\
\text { completers after } 2 \text { years) }\end{array}$ \\
\hline $\begin{array}{l}\text { Incomplete outcome data (attrition bias) } \\
\text { Objective outcomes }\end{array}$ & High risk & $\begin{array}{l}\text { Comment: ITT performed, however, high } \\
\text { numbers not completing ( } 35 \% \text { to } 51 \% \\
\text { completers after } 2 \text { years) }\end{array}$ \\
\hline Selective reporting (reporting bias) & High risk & $\begin{array}{l}\text { Comment: only the 24-month results are } \\
\text { shown }\end{array}$ \\
\hline Other bias & Unclear risk & $\begin{array}{l}\text { Comment: not enough information to } \\
\text { judge }\end{array}$ \\
\hline
\end{tabular}


Methods

Participants
Parallel randomised controlled trial

Randomisation ratio: not reported

Non-inferiority design: 1 -sided confidence interval

Inclusion criteria: parents and their overweight or obese ( $>85$ th BMI percentile) children, aged 8-12 years. At least 1 parent or guardian participated with the child. If 2 children in the family met criteria for the study, both were invited to attend the treatment groups but a coin-flip was used to determine which child's data would be part of the study

Exclusion criteria: either the child or parent currently involved in psychological or weight loss treatment, using medications that affected weight or appetite, or had a psychiatric or physical condition (e.g. eating disorder, psychosis) that would interfere with participation

Diagnostic criteria: as above ( $>85$ th BMI percentile)
Number of study centres: 2

Treatment before study: none

Titration period: none

Intervention description:

Parent-only:

Behavioural change skills; included self monitoring of targeted behaviours, positive reinforcement, stimulus control, pre-planning and modelling. Parents in the parent-only group were coached on how to assist their children in weight monitoring at home and reflect on the behaviours that influenced weight. Goal setting for the parent-only group occurred during the treatment groups. Completed quizzes each week to assure knowledge of the treatment protocol. The intervention was theoretically based: using a current state-of-the-art behavioural treatment for childhood obesity described by Epstein and colleagues (references provided)

All interventionists attended a 3-day training regarding the behavioural intervention for the study, and were supervised by the first author on a weekly basis during treatment. The intervention was provided by a psychologist

Parent-child intervention:

The material taught in the child groups was similar in content to that taught to the parents and described above, but presented in an age-appropriate manner (i.e. fun games) . Parent-child dyads also met with an interventionist either pre- or post group for family goal setting for a maximum of 10 minutes. All parents and children completed quizzes each week to assure knowledge of the treatment protocol

Components of the interventions in common between the groups included:

- 20-week intervention, number of sessions not reported, each session was 60 minutes

- A physical activity component: energy expenditure was addressed by increasing physical activity (lifestyle and planned) and decreasing sedentary behaviour

- A nutritional advice component (traffic-light diet): following the food guide pyramid and focusing on decreasing energy intake while increasing the nutrient density of the diet

- No medication component was included

- No financial incentives

Outcomes

Outcomes reported in abstract of publication: inferiority of treatment group on child weight loss, parent weight loss and child physical activity, caloric intake 
Boutelle 2011 (Continued)

\begin{tabular}{ll}
\hline Study details & $\begin{array}{l}\text { Run-in period: none } \\
\text { Study terminated before regular end (for benefit/because of adverse events): no }\end{array}$ \\
\hline Publication details & $\begin{array}{l}\text { Language of publication: English } \\
\text { Non-commercial funding } \\
\text { Publication status: peer-reviewed journal }\end{array}$ \\
\hline Stated aim for study & $\begin{array}{l}\text { Quote from publication: "to evaluate whether a standardized behavioral parent only } \\
\text { treatment program would not be inferior to a standardized behavioral parent-child pro- } \\
\text { gram on child weight loss and other relevant markers of change" }\end{array}$ \\
\hline Notes & \\
\hline Risk of bias &
\end{tabular}

\begin{tabular}{|c|c|c|}
\hline Bias & Authors' judgement & Support for judgement \\
\hline $\begin{array}{l}\text { Random sequence generation (selection } \\
\text { bias) }\end{array}$ & Low risk & $\begin{array}{l}\text { Comment: } 80 \text { parent-child dyads were ran- } \\
\text { domly assigned to either parent-child or } \\
\text { parent-only groups. Random assignment } \\
\text { was conducted after completing the ini- } \\
\text { tial assessment using a computer-generated } \\
\text { random numbers table }\end{array}$ \\
\hline Allocation concealment (selection bias) & Unclear risk & Comment: no further details \\
\hline $\begin{array}{l}\text { Blinding of participants and personnel } \\
\text { (performance bias) } \\
\text { Objective outcomes }\end{array}$ & Unclear risk & Comment: no details provided \\
\hline
\end{tabular}

Blinding of outcome assessment (detection Unclear risk

Comment: no details provided

bias)

Objective outcome

Incomplete outcome data (attrition bias) High risk

Objective outcomes

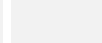

Run-in period: none

Language of publication: English

Non-commercial funding

Quote from publication: "to evaluate whether a standardized behavioral parent only oral parent-child pro-

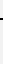


Boutelle 2011 (Continued)

$\begin{array}{lll}\text { Other bias } & \text { Unclear risk } & \begin{array}{l}\text { Comment: not enough information to } \\ \text { judge }\end{array}\end{array}$

West 2010

$\begin{array}{ll}\text { Methods } & \text { Parallel randomised controlled trial } \\ \text { Randomisation ratio: not reported } \\ \text { Superiority design }\end{array}$

Participants

Inclusion criteria: parent described the child's body size as overweight, the child was 411 years of age, and the parent agreed to attend a 12-week intervention

Exclusion criteria: child taking medication that affected growth or weight control, or had a severe developmental delay or disability

Diagnostic criteria: parental description of child as overweight

Interventions

Number of study centres: 6

Treatment before study: none

Titration period: none

Description of the intervention:

Parent-only:

12-week intervention, 1 session per week. Group Lifestyle Triple P is a modification of Level 4 Group Triple P tailored to the concerns of parents of overweight and obese children. The intervention is a 12 -week intervention that consists of $9 \times 90$-minute group sessions and $3 \times 20$-minute telephone sessions. To help parents acquire new knowledge and skills, all sessions used an active skills training process (e.g. demonstrating and rehearsing skills) within a self regulation framework (e.g. self selecting goals and self evaluating progress). Each parent received a workbook summarising the session content, and suggested between-session tasks

Group sessions: in the first group session, motivational interviewing techniques were used to enhance parents' commitment to change. During subsequent group sessions, a range of specific strategies were introduced and practised. These included: positive parenting strategies (e.g. keeping track of children's lifestyle behaviour, setting clear guidelines about food and activity, reinforcing healthy behaviour). Also physical activity strategies (e.g. reducing TV and computer time, increasing energetic play, encouraging involvement in sport) and nutrition strategies (e.g. establishing eating routines, modifying recipes, reading food labels) were introduced and practised

Telephone sessions: during the telephone sessions, the facilitator reviewed parents' implementation of strategies, and problem-solved any difficulties. The final group session covered progress review and maintenance of treatment gains

Triple P programme was standardised. All sessions were facilitated by a clinical psychologist and accredited provider of Group Triple P (who co-authored the intervention materials), with assistance from graduate students in nutrition and dietetics, physical education and psychology

Control:

Waiting list control for 12 weeks. Included a physical activity and nutritional advice components

Parent-only interventions for childhood overweight or obesity in children aged 5 to I I years (Review) 
West 2010 (Continued)

\begin{tabular}{l|l} 
Outcomes & $\begin{array}{l}\text { Outcomes reported in abstract of publication: child BMI z score and weight-related } \\
\text { problem behaviour, confidence in managing children's weight-related behaviour }\end{array}$ \\
\hline Study details & $\begin{array}{l}\text { Run-in period: none } \\
\text { Study terminated before regular end (for benefit/because of adverse events): no }\end{array}$ \\
\hline Publication details & $\begin{array}{l}\text { Language of publication: English } \\
\text { Non-commercial funding } \\
\text { Publication status: peer-reviewed journal }\end{array}$ \\
\hline Stated aim for study & $\begin{array}{l}\text { Quote from publication: “..to evaluate the effects of the intervention on parenting and } \\
\text { child weight-related behaviour, relative to a waiting list control condition. This study } \\
\text { describes the evaluation of a lifestyle-specific parenting program (Group Lifestyle Triple } \\
\text { P) on multiple child and parent outcomes...” }\end{array}$ \\
\hline
\end{tabular}

Notes

Risk of bias

\begin{tabular}{|c|c|c|}
\hline Bias & Authors' judgement & Support for judgement \\
\hline $\begin{array}{l}\text { Random sequence generation (selection } \\
\text { bias) }\end{array}$ & Low risk & $\begin{array}{l}\text { Quote from publication: "Families were } \\
\text { randomly allocated to either the interven- } \\
\text { tion...or the waiting list control...Groups } \\
\text { were allocated to conditions according to } \\
\text { a list of computer generated random num- } \\
\text { bers..." }\end{array}$ \\
\hline Allocation concealment (selection bias) & High risk & $\begin{array}{l}\text { Quote from publication: "Allocation con- } \\
\text { cealment and blinded outcome assessment } \\
\text { were not possible due to limited staff and } \\
\text { resources" } \\
\text { Comment: no masking of allocation to in- } \\
\text { tervention or control }\end{array}$ \\
\hline $\begin{array}{l}\text { Blinding of participants and personnel } \\
\text { (performance bias) } \\
\text { Subjective outcomes }\end{array}$ & High risk & Comment: no masking \\
\hline $\begin{array}{l}\text { Blinding of participants and personnel } \\
\text { (performance bias) } \\
\text { Objective outcomes }\end{array}$ & High risk & Comment: no masking \\
\hline $\begin{array}{l}\text { Blinding of outcome assessment (detection } \\
\text { bias) } \\
\text { Subjective outcomes }\end{array}$ & High risk & Comment: no masking \\
\hline
\end{tabular}

Parent-only interventions for childhood overweight or obesity in children aged 5 to I I years (Review) 
West 2010 (Continued)

\author{
Blinding of outcome assessment (detection High risk \\ Comment: no masking \\ bias) \\ Objective outcome
}

Incomplete outcome data (attrition bias) Low risk

Subjective outcomes

Comment: numbers missing reported and reasons explained; ITT analysis with the

last point-carried-forward

Incomplete outcome data (attrition bias) Low risk

Objective outcomes

Comment: numbers missing reported and reasons explained; ITT analysis with the last point-carried-forward

Selective reporting (reporting bias) Unclear risk

Comment: all outcomes reported as stated but not enough information to judge

Other bias $\quad$ Unclear risk

Comment: not enough information to judge

Resnick 2009

Methods

Parallel randomised controlled trial

Randomisation ratio: not reported

Superiority design

Participants

Inclusion criteria: eligible parents must have had a child: (a) with a BMI $\geq 85$ th percentile, (b) enrolled at 1 of the 2 study schools, (c) between Grades K and 5, (d) parents also had to agree to be randomised to either 1 of the 2 study conditions. In instances when families had multiple children enrolled at a study school with BMIs $\geq$ 85th percentile, the oldest child was considered the index child

Exclusion criteria: none stated

Diagnostic criteria: 85 th percentile BMI

Interventions

Number of study centres: 2

Treatment before study: none

Titration period: none

Description of interventions:

Focus groups with 9 parents helped to determine study content, messages, and potential use of the 2 delivery methods, 1-to-1 counselling and mailed materials

Educational mailed materials:

Posted approximately every 5 weeks, 6 mailings over 30 weeks

The educational material included: tips to increase walking, talk with children about TV viewing. Received a physical activity book and a pedometer. Read nutrition labels, shop more healthfully at grocery stores, talk with children about eat out healthfully. Received a cookbook, had a hands-on activity about portion sizes

Educational material plus personal encounters:

Received the same educational materials as above

The type of encounter (home visit, telephone call, etc.) was based on participating

Parent-only interventions for childhood overweight or obesity in children aged 5 to I I years (Review)

Copyright $\odot 2015$ The Cochrane Collaboration. Published by John Wiley \& Sons, Ltd. 
Resnick 2009 (Continued)

parents' preferences and schedules. Parents selected the topics discussed during each visit from choices provided by community health workers (CHWs) (i.e. epidemiology of childhood overweight, biological, social and environmental influences on childhood overweight; basic nutrition; label reading; grocery shopping strategies, including a tour of a local grocery store; physical activity guidelines)

Mean 3.4 personal encounters over the 30 weeks. On average, parents received encounters for 18 weeks

Both interventions were delivered by CHWs who attended a 36-hour training programme over the course of 6 days. The purpose of the training was to prepare CHWs to make evidence-based recommendations to families such as changing to reduced fat milk, reducing the intake of snack foods, replacing sugar-sweetened beverages with water, increasing fruit and vegetable intake, decreasing TV viewing and increasing physical activity. The topics covered during the training included guidelines for home visits; the epidemiology of childhood overweight, biological, social and environmental influences on childhood overweight; basic nutrition; label reading; grocery shopping strategies, including a tour of a local grocery store; physical activity guidelines and counselling strategies. CHWs practiced counselling skills during their training by engaging in role-plays with each other. After training and throughout the study, the study staff and CHWs met monthly to discuss specific concerns or difficulties with study participants

Outcomes

Study details

Publication details

Stated aim for study

Notes

\section{Risk of bias}

Bias

Random sequence generation (selection Unclear risk bias)
Outcomes reported in abstract of publication: BMI

Run-in period: none

Study terminated before regular end (for benefit/because of adverse events): no

Language of publication: English

Non-commercial funding

Publication status: peer-reviewed journal

Quote from publication: "The goal was to create an easy-to-use parent outreach model that could ultimately be used by school nurses, paediatricians, community health agencies, and CHWs"

$\begin{array}{ll} & \begin{array}{l}\text { pate in one of two study groups and were } \\ \text { randomised to either one of the two study } \\ \text { conditions...” }\end{array} \\ \text { Comment: no description of randomisa- } \\ \text { tion process }\end{array}$


Resnick 2009 (Continued)

Blinding of participants and personnel Unclear risk

Comment: no details provided

(performance bias)

Objective outcomes

Blinding of outcome assessment (detection Unclear risk

bias)

Objective outcome

Incomplete outcome data (attrition bias) High risk

Objective outcomes

Comment: no ITT analysis. In all, 42/

$46(91 \%)$ parents completed post-inter-

vention surveys

Selective reporting (reporting bias) Unclear risk

Comment: all outcomes reported as stated but not enough information to judge

Other bias

Unclear risk

Comment: not enough detail to judge

Estabrooks 2009

Methods

Parallel randomised controlled trial

Randomisation ratio: not reported

Superiority design

Participants

Inclusion criteria: $\mathrm{BMI} \geq 85$ th percentile for their age

Exclusion criteria: plans to move out of the state during the course of the study, a request by the child's paediatrician that the family not be contacted

Diagnostic criteria: $\mathrm{BMI} \geq 85$ th percentile for their age

Interventions

Number of study centres: not reported

Treatment before study: not reported

Titration period: not reported

Description of interventions:

Based on social-ecological theory (reference provided). 3 intervention groups: workbook, group sessions, group sessions + interactive voice response (IVR) counselling

Parent group + IVR counselling:

Group sessions as above. Then 10 follow-up sessions by IVR, 1 per week. Included behavioural, physical activity and nutritional components (12-week intervention)

Physical activity get at least 2.5 hours of moderate physical activity this week; make a list of your family's barriers to physical activity around the house, and come up with a way to overcome them; do something active with your child for 15 minutes at least 3 times this week; review the family goal sheet with your family and set measurable, specific and objective goals this week; decrease your sitting time by 0.5 hours each day this week; increase your physical activity by 1 day per week; set a goal to take your family to a new park or trail that you have not visited before; survey your family regarding physical activity that they would like to do as a family, and try to do that activity at least 3 times per week

Nutrition parent healthy habits: set a good example for your child by eating 5-9 servings

Parent-only interventions for childhood overweight or obesity in children aged 5 to I I years (Review) 
Estabrooks 2009 (Continued)

of fruits and vegetables every day this week; drink low-fat milk at 1 meal each day; clear the kitchen cupboards of unhealthy snacks; prepare at least 1 healthy meal together with your child; post signs at least twice this week about your family's positive changes with healthy eating; decrease your soda and sugared-drink consumption by 1 serving per day; increase your servings of fruits and vegetables by 3 per day; check your library for cookbooks, recipes or videos that help your family to prepare nutritious meals; change 1 food item that is high-fat to a healthy snack of fruit or vegetable

Behavioural consistency and contingency; communication; praise; parenting skills; support; plan; commitment

Group sessions were led by a dietician (no further details)

Parent group sessions:

2 group sessions. Utilised the workbook used in the control condition. Addressed parents' behavioural health skills and knowledge of weight, nutrition and physical activity. It also identified key parenting skills: limit setting, effective communication and role modelling. This session concluded with role playing, problem solving and the development of an action plan. 24-week intervention

Parent workbook group:

61-page workbook to promote physical activity and the consumption of fruits and vegetables and decrease sugared-drink consumption and TV viewing/recreational computer time. Activity to explore parental beliefs about eating and physical activity, healthy habits for creating a healthy family, defining the division of responsibility for eating and activity. Physical activity: using FITT (frequency, intensity, time, type) principles. Nutrition: helping children to avoid fad diets, reading labels, selecting healthy food options, sample menus, tips for preparing healthy snacks and meals. Assessing and calculating BMI in children and adults, causes of overweight in children (biological, cognitive, environmental), 5 reasons children gain weight, impact of being overweight, parenting skills to support weight reduction, survey of the family home environment, ways to promote a healthy home environment, goal setting: creating a family action plan, process of goal setting and keeping objectives clear, parent's personal action plan, barriers and strategies to maintaining family action plan. 24-week intervention

Outcomes

Outcomes reported in abstract of publication: child BMI z scores, symptoms of eating disorders and body image

Study details

Run-in period: not reported

Study terminated before regular end (for benefit/because of adverse events): not reported

Publication details

Language of publication: English

Non-commercial funding

Publication status: peer-reviewed journal

Stated aim for study

Quote from publication: “A practical RCT [randomised controlled trial] evaluated the relative effectiveness of three interventions to support parents of overweight or at-risk children to change the home environment to foster more healthful child eating and activity behaviours, thereby reducing child BMI and BMI z scores. A secondary purpose was to determine the patterns of use and potential dose effect for the highest-intensity intervention"

Parent-only interventions for childhood overweight or obesity in children aged 5 to II years (Review) 
Estabrooks 2009 (Continued)

Notes

There were 3 comparisons of relevance to this review: the parent-only group and IVR vs. the control (workbook); the parent-only group vs. the control; the parent-only group and IVR vs. the parent group

\section{Risk of bias}

\begin{tabular}{|c|c|c|}
\hline Bias & Authors' judgement & Support for judgement \\
\hline $\begin{array}{l}\text { Random sequence generation (selection } \\
\text { bias) }\end{array}$ & Low risk & $\begin{array}{l}\text { Quote from publication: "Through a ran- } \\
\text { dom-numbers table, participants were as- } \\
\text { signed randomly (families/staff unblinded) } \\
\text { to the FC-workbook, the FC-group, or the } \\
\text { FC-IVR intervention..." }\end{array}$ \\
\hline Allocation concealment (selection bias) & Unclear risk & Comment: no details \\
\hline $\begin{array}{l}\text { Blinding of participants and personnel } \\
\text { (performance bias) } \\
\text { Subjective outcomes }\end{array}$ & High risk & $\begin{array}{l}\text { Comment: families/staff were both un- } \\
\text { blinded }\end{array}$ \\
\hline
\end{tabular}

Blinding of participants and personnel High risk (performance bias)

Comment: families/staff were both un-

Objective outcomes

blinded

Blinding of outcome assessment (detection High risk

bias)

Comment: families/staff were both unblinded

Subjective outcomes

Blinding of outcome assessment (detection Low risk bias)

Objective outcome

Incomplete outcome data (attrition bias) Low risk Subjective outcomes

Incomplete outcome data (attrition bias) High risk Objective outcomes
Comment: families/staff were both unblinded, but probably no substantial impact on outcome measures

Comment: study says they carried out ITT analysis; however, results were only given for completers. $72 \%$ in workbook intervention, $66 \%$ in group intervention and $74 \%$ in group + IVR intervention completed 12 month follow-up - drop-outs rates average for this length of follow-up

Comment: study says they carried out ITT analysis; however, results were only given for completers. $72 \%$ in workbook intervention, $66 \%$ in group intervention and $74 \%$ in group + IVR intervention completed 12month follow-up - drop-out rates average for this length of follow-up 
Estabrooks 2009 (Continued)

Selective reporting (reporting bias)

High risk

Unclear risk

Other bias
Comment: states health-related quality of life reported but no data reported
Comment: unsure if all the participants stayed in their randomised groups

Munsch 2008

Methods

Participants

\section{Parallel randomised controlled tria \\ Randomisation ratio: not reported Equivalence design}

Inclusion criteria: $\mathrm{BMI}>85$ th percentile adjusted for gender and age. Participating family members were able to speak and write in German. All participants were free from diabetes, heart disease and endocrine disorders

Exclusion criteria: parents and children meeting the criteria of the DSMIV-TR for mental disorders warranting immediate treatment (assessed in a clinical interview), such as suicidal tendency, psychosis, mania, organic dementia or substance abuse disorder. Parents' or children's participation in a diet programme or other psychotherapy treatment with weight loss medication. There were only 4 fathers eligible for treatment, therefore, excluded from the analyses

Diagnostic criteria: $\mathrm{BMI}>85$ th percentile adjusted for gender and age

\section{Number of study centres: 2 \\ Treatment before study: none \\ Titration period: no}

Description of interventions: the TAKE (Training adipöser Kinder und ihrer Eltern) programme. Programme overall proceeded with group sessions that began with a short overview of the topic, then individual difficulties and progress with homework were discussed and the group established coping strategies. Afterwards the major topics of the sessions were implemented and new homework was assigned. Interventions throughout the programme were highly interactive, proceeded step by step, and involved the group as a whole as well as individual mothers and children (where appropriate)

Mother's CBT:

10 sessions in 5 phases over 10 weeks (120-minute sessions)

Phase 1 (nutrition and eating behaviour, covered across 8 sessions including psychoeducation about childhood obesity, risks, nutritional counselling, goal setting, stimulus control, family rules); phase 2 (physical activity, covered within 2 sessions including psychoeducation about physical activity, motivation, suitable sports); phase 3 (social competences, covered in 1 session, including social skills training, parental modelling and support of children dealing with difficulty situations); phase 4 (body concept, covered in 1 session, included supporting child's developments of a positive body concept); phase 5 (relapse prevention, covered in 1 session, included training of maintenance skills, appraisal of goal attainment, developing coping strategies). Children attended a relaxation training (progressive muscle relaxation training) of equal frequency and duration to the disorder-specific CBT of children in the mother-child group

Mother-child arm: 
10 weekly treatment sessions of 120 minutes. Mothers received CBT including the same components as the Mothers-only group. Children received sessions on nutrition and eating behaviour, basic nutritional education, reinforcement and tokens, lessons in physical activity, social competencies (self assertiveness, social skills, saying 'no' to food offers, role modelling, anti-bullying plans), developing a positive body concept and relapse prevention

Sessions were undertaken by psychologists and trained co-therapists. All therapists were trained and supervised weekly by 1 of the authors

Outcomes

Outcomes reported in abstract of publication: per cent overweight, general behaviour problems (externalising and internalising behaviour problems), global and social anxiety, and depression

Study details

Run-in period: none

Study terminated before regular end (for benefit/because of adverse events): no

Publication details

Language of publication: English

Non-commercial funding

Publication status: peer-reviewed journal and Word document

Stated aim for study

Quote from publication: "we investigated whether the treatment of parents only would be as efficacious as a parent-child treatment in a randomised controlled clinical trial. Our group treatment approach, TAKE [Training adipöser Kinder und deren Eltern ('training of obese children and their parents')], targeted weight stabilization and reduction of behavioral problems of obese children aged 8-12 years"

Notes

Risk of bias

$\begin{array}{ll}\text { Bias Authors' judgement } & \text { Aud }\end{array}$

Random sequence generation (selection Unclear risk bias)

\section{Support for judgement}

Quote from publication: "families were randomly assigned according to a permuted block design to either the mother-child (condition A) or the mother-only (condition B) cognitive behavioral therapy (CBT) treatment..."

Comment: no details provided

Comment: no details provided

Blinding of participants and personnel Unclear risk (performance bias)

Subjective outcomes

Blinding of participants and personnel Unclear risk (performance bias)

Objective outcomes
Comment: no details provided 
Munsch 2008 (Continued)

\begin{tabular}{l|l|l|l}
\hline $\begin{array}{l}\text { Blinding of outcome assessment (detection } \\
\text { bias) } \\
\text { Subjective outcomes }\end{array}$ & Unclear risk & Comment: no details provided \\
\hline
\end{tabular}

Blinding of outcome assessment (detection Unclear risk Comment: no details provided

bias)

Objective outcome

Incomplete outcome data (attrition bias) High risk

Subjective outcomes

Incomplete outcome data (attrition bias) High risk

Objective outcomes

Selective reporting (reporting bias) Unclear risk

Other bias

Unclear risk
Comment: high rates of drop-out (motherchild 11/31 dropped out, in mother-only 18/25 dropped out), no ITT analysis

Comment: high rates of drop-out (motherchild 11/31 dropped out, in mother-only 18/25 dropped out), no ITT analysis

Comment: all outcomes reported as stated but not enough information to judge

Comment: families were randomly assigned according to a permuted block design to either the mother-child (condition A) or the mother-only (condition B) CBT treatment. Selection bias: there were only 4 fathers eligible for treatment and they were excluded from the analyses. Also underpowered

Janicke 2008

Methods

Participants

\section{Parallel randomised controlled tria \\ Randomisation ratio: not reported \\ Superiority design}

Inclusion criteria: $\mathrm{BMI} \geq 85$ th percentile for age and gender, required to live within the same dwelling in a rural county that is designated in whole or in part as a "Health Professional Shortage Areas" by the U.S. Department of Health and Human Services, to obtain physician approval to participate in the study, with documentation provided via letter signed by a physician medically clearing individuals to participate in a weight management programme

Exclusion criteria: child or participating parent has a dietary or exercise restriction, or a medical condition that contraindicates mild energy restriction or moderate physical activity (including a history of musculoskeletal condition that limits walking; heart condition; chronic lung diseases limiting physical activity; uncontrolled diabetes; uncontrolled hypertension; thyroid disease; or uncontrolled exercise-induced asthma as determined by a physician). Children or participating parents on antipsychotic agents, systemic corticosteroids or who were currently using prescription weight-loss drugs, insulin or other 
diabetic medications. Not engaged in another weight control programme, exhibit conditions or behaviours that were likely to affect their participation in the trial, such as being unwilling or unable to give informed consent, parent(s) or legal guardian(s) unable to read English at approximately the 5th grade level, unwilling to accept random assignment, unable to travel to extension office for intervention sessions, or likely to move out of the county within the next 12 months

Diagnostic criteria: $\mathrm{BMI} \geq 85$ th percentile for age and gender

Interventions

\section{Number of study centres: not reported \\ Treatment before study: none \\ Titration period: none \\ Detailed description of interventions}

Behavioural parent-based intervention:

12 sessions of 90 minutes

Only the participating parent(s) attended group meetings. The primary treatment objectives were to build healthier dietary habits, increase moderate intensity physical activity, establish a healthier weight status and build positive self worth

Each session included 3 segments, similar to the parent group for the Family-Based intervention. An emphasis was placed on teaching parents how to work with their children to set goals. Each week interventionists suggested a range of dietary and physical activity targets that would be appropriate for each child and parent. Parents were encouraged to meet with their children to set individual goals within the suggested range

Increased physical activity encouraged through a pedometer-based step programme as described above

Changes in dietary habits were addressed via a modified version of the Stoplight Diet Set daily dietary goals at the end of each group session, which included limiting the consumption of high-fat/high-sugar foods (i.e. "red foods") and increasing fruit and vegetable intake. Encouraged to eat a well-balanced diet based on the food guide pyramid Parents participated in role-play activities to practice negotiation of goals with their child. As children were not attending group sessions, an emphasis was placed on teaching parents how to work with their children to set goals together. Parents encouraged to utilise praise, incentives and modelling to encourage participation and goal achievement. Parents provided handouts to guide them in discussing programme material and setting weekly goals with their children. Parents weighed every other group session to monitor their weight status

Delivered as described below

Behavioural family-based intervention:

12 sessions of 90 minutes. Parent-child dyads participated in simultaneous but separate groups. The primary objectives were to build healthier dietary habits, increase moderate intensity physical activity, establish a healthier weight status and build positive self worth 1) In the parent group: the first portion of the meeting involved a review of the progress made in implementing the strategies developed for changing their eating and exercise habits. Difficulties reported by the parents were addressed through group support and discussion. The second segment focused on knowledge and skill training related to nutrition, physical activity and behaviour management strategies

At the end of each session, children and parents were brought together to develop goals for the week and specific plans to achieve these goals

2) The child group sessions included 3 segments: a review of progress during the previous 
week, a physical activity to demonstrate strategies to keep active and preparation of a healthy snack

Increased physical activity encouraged through a pedometer-based step programme. Children and parents encouraged to monitor their physical activity and gradually increase their steps per day. Programme goals based on their baseline level of steps and targeting an increase of at least 3000 steps per day by the end of the programme. Goals set for gradually decreasing sedentary activities (children spend no more than 2 hours per day watching TV or playing video games). If excessive TV viewing was not a concern for a given family, group leaders targeted non-homework-based computer time

Changes in dietary habits addressed via a modified version of the Stop-Light programme. Child and parent participants monitored everything they ate using a daily habit log. Goals were individualised to the needs of each family and based on each individual's baseline dietary intake and progress (i.e. goal attainment, weight change) over the course of the programme. Daily dietary goals set each week, including limiting the consumption of high-fat/high-sugar "red foods" (with an absolute minimum goal of 2 red foods per day), and increasing fruit and vegetable intake. Children and adults encouraged to eat a well-balanced diet based on the food guide pyramid

Incentivised by providing payment for transportation costs (USD 5 per session) and USD 50 for completing post-treatment and 6-month assessment visits

Delivered by Family and Consumer Sciences Agents, in collaboration with a post-doctoral clinical psychologist and graduate students in clinical health psychology who had extensive training and certification in the treatment protocols. The principal investigator of the study conducted periodic direct observation of group sessions to monitor interventionist's performance and assess treatment fidelity. The interventionists also participated in weekly supervision meetings to review each family's progress, discuss group interactions and prepare for the next group session

Waiting list control:

Families assigned to the waiting list control condition completed the assessment protocol at baseline, and at 4 and 10 months. After the follow-up period (month 10), families were invited to participate in a 12 -session behavioural-based intervention. No treatment was delivered until after the final, 6-month follow-up assessment

Publication details

Language of publication: English

Non-commercial funding

Publication status: peer-reviewed journal

Quote from publication: "The goals of the study are to (a) assess the feasibility of recruitment in rural settings, (b) develop and evaluate training protocol for group leaders, (c) determine strategies to increase adherence to monitoring and goal setting protocol, (d) evaluate strategies for participant retention, (e) assess the relative cost-effectiveness of the interventions, (f) assess the acceptability of the intervention to families and Cooperative Extension administrators and personnel, and (g) if successful, estimate the sample size needed for a full scale trial" 
Janicke 2008 (Continued)

Notes

There were 2 comparisons of relevance to this review: the parent-only vs. parent-child group and the parent-only vs. the waiting list control group

Risk of bias

Bias Authors' judgement $\quad$ Support for judgement

Random sequence generation (selection Unclear risk bias)

Comment: during the initial telephone screen, families were informed as to which 2 evenings the group intervention sessions would be held in their county. Families then indicated which of these evenings they were available to attend. Before the baseline assessment, all families were randomised via computer assignment, based on availability, to 1 of the 2 specific week nights or the waiting list control condition. After randomisation of all families, the interventions (parent-only or family based) were assigned randomly to the specific week nights

Allocation concealment (selection bias) Unclear risk

Quote from study author: "Randomization was conducted by a research team member who did not participate in assessments. Assignments were written down and put in an envelope by the person making the assignments for each dyad. Envelope was opened with family at end of baseline visit"

Comment: unclear whether envelopes were opaque

Blinding of participants and personnel High risk (performance bias)

Quote from study author: "Participants and treatment personnel were not blind" Comment: families were notified of their group assignment at pre-treatment assessment

Blinding of participants and personnel High risk (performance bias)

Quote from study author: "Participants and treatment personnel were not blind" Comment: families were notified of their group assignment at pre-treatment assessment

Blinding of outcome assessment (detection Unclear risk bias)

Subjective outcomes

Quote from study author: "Outcome assessors were blinded in that we used personnel to complete outcome assessments that did not serve as treatment personnel in each respective county"

Parent-only interventions for childhood overweight or obesity in children aged 5 to I I years (Review)

Copyright $\Subset 2015$ The Cochrane Collaboration. Published by John Wiley \& Sons, Ltd. 


\begin{tabular}{|c|c|c|}
\hline $\begin{array}{l}\text { Blinding of outcome assessment (detection } \\
\text { bias) } \\
\text { Objective outcome }\end{array}$ & Unclear risk & $\begin{array}{l}\text { Quote from study author: "Outcome asses- } \\
\text { sors were blinded in that we used personnel } \\
\text { to complete outcome assessments that did } \\
\text { not serve as treatment personnel in each re- } \\
\text { spective county" }\end{array}$ \\
\hline $\begin{array}{l}\text { Incomplete outcome data (attrition bias) } \\
\text { Subjective outcomes }\end{array}$ & High risk & $\begin{array}{l}\text { Comment: no ITT analysis. Numbers } \\
\text { completing assessment provided only }\end{array}$ \\
\hline $\begin{array}{l}\text { Incomplete outcome data (attrition bias) } \\
\text { Objective outcomes }\end{array}$ & High risk & $\begin{array}{l}\text { Comment: no ITT analysis. Numbers } \\
\text { completing assessment provided only }\end{array}$ \\
\hline Selective reporting (reporting bias) & High risk & $\begin{array}{l}\text { Comment: collected other measures that } \\
\text { were not reported }\end{array}$ \\
\hline Other bias & Unclear risk & $\begin{array}{l}\text { Comment: not enough information to } \\
\text { judge }\end{array}$ \\
\hline
\end{tabular}

Golley 2007

Methods

Participants

\section{Parallel randomised controlled trial \\ Randomisation ratio: not reported \\ Superiority design}

Inclusion criteria: overweight (according to the International Obesity Task Force definition), and Tanner stage with a carer willing to attend sessions and able to read and understand English

Exclusion criteria: BMI z score $>3.5$, diagnosed with a syndromal cause of obesity, using medications that influence weight gain or loss, a diagnosis of physical or developmental disability or chronic illness, and a sibling enrolled in the study

Diagnostic criteria: overweight (defined as above)

\section{Number of study centres: 2 \\ Treatment before study: none \\ Titration period: none \\ Detailed description of interventions:}

Parenting-skills training:

11 sessions over 24 weeks, 4 weekly 2 -hour group sessions followed by 4 weekly, then 3 monthly 15 - to 20 -minute individual telephone sessions. Parenting-skills training was used to facilitate and support parents to undertake family lifestyle change. Positive, Parenting Program (Triple P) based on child development theory and social learning principles and aimed to promote parental competence to manage their child's behaviour. Standard Triple P resource materials were used with programme examples adapted to reflect dietary and activity behaviours. Application of Triple $P$ to eating and activity behaviours was supported by provision of a general healthy lifestyle pamphlet

The Triple P Selected Seminar Series consisted of $3 \times 2$-hour seminars covering: 1. positive parenting; 2. raising confident and competent-children, and; 3 . raising resilient-children 
Learning outcomes - to practitioners (which some is applicable to parents)

- Core principles of positive parenting and behaviour change

- Specific positive parenting strategies for promoting children's development

- Responding to parents' questions

- Early detection and effective management of child behaviour problems

- Core principles of positive parenting and behaviour change

- Factors influencing child behaviour

- Specific positive parenting strategies for promoting children's development

- Effective parent consultation

- Identification of indicators suggesting more intervention is required and appropriate referral procedures

Triple P Discussion Groups topics are:

- hassle-free shopping with children

- managing fighting and aggression

- developing good bedtime routines

- dealing with disobedience

Intervention delivered using standard protocols and a single, trained facilitator to limit site bias and enhance internal study validity. Sessions taken by a dietician

Parenting-skills training with intensive lifestyle education:

As above in addition to: 7 intensive lifestyle support group sessions that focused on lifestyle knowledge and skills including the following: family-focused healthy eating with specific core food serve recommendations, monitoring, label reading, snacks, modifying recipes, being active in a variety of ways, roles and responsibilities around eating, managing appetite, self esteem and teasing

While parents attended the lifestyle sessions, children in the group attended structured, supervised activity sessions developed by physical activity experts. The sessions consisted of fun, non-competitive games designed around aerobic activity and development of fundamental motor skills. Sessions were designed as play rather than exercise and were diversional rather than interventional. The activities required minimal equipment and were deliverable by non-expert staff and easily replicated at home. Sessions taken by a dietician

Waiting list control:

Received the same general healthy-lifestyle pamphlet as the parenting-alone group. During the 12-month waiting list period, the waiting list control group was contacted by telephone 3 or 4 times for 5 minutes as a retention strategy. Researcher contact with the waiting list families was minimised to avoid the potential placebo effect of therapist contact 
Golley 2007 (Continued)

\begin{tabular}{ll}
\hline Stated aim for study & $\begin{array}{l}\text { Quote from publication: "Our goal was to evaluate the relative effectiveness of parenting- } \\
\text { skills training as a key strategy for the treatment of overweight children. The aim of } \\
\text { this study was to evaluate the relative effectiveness of parenting-skills training as a key } \\
\text { strategy for the treatment of overweight children" }\end{array}$ \\
\hline Notes & $\begin{array}{l}\text { There were } 3 \text { comparisons of relevance to this review: the parent-only intensive education } \\
\text { group vs. the parent-only group; the parent-only + intensive education group vs. the } \\
\text { waiting list control; the parent-only group vs. the waiting list control }\end{array}$ \\
\hline
\end{tabular}

Risk of bias

\begin{tabular}{|c|c|c|}
\hline Bias & Authors' judgement & Support for judgement \\
\hline $\begin{array}{l}\text { Random sequence generation (selection } \\
\text { bias) }\end{array}$ & Low risk & $\begin{array}{l}\text { Quote from publication: "Randomization } \\
\text { schedules were computer generated using a } \\
\text { 3-block design stratified for gender and site } \\
\text { of recruitment..." } \\
\text { Comment: researchers involved in recruit- } \\
\text { ment, participant allocation and interven- } \\
\text { tion delivery or data collection were not in- } \\
\text { volved in the randomisation process }\end{array}$ \\
\hline Allocation concealment (selection bias) & Low risk & $\begin{array}{l}\text { Quote from publication: "Individual group } \\
\text { allocations were sealed in opaque en- } \\
\text { velopes, with the next envelope opened on } \\
\text { a child's completion of baseline measure- } \\
\text { ments..." }\end{array}$ \\
\hline
\end{tabular}

Blinding of participants and personnel Unclear risk (performance bias)

Comment: carers and participants masked Objective outcomes to allocation to treatment assignment but unclear if this also related to blinding throughout the trial

Blinding of outcome assessment (detection Low risk bias)

Objective outcome
Quote from publication: “...Data collection was performed by the same trained assessor who was blinded to participant group allocation"

Comment: outcome assessment was masked

Quote from publication: "Intention-totreat analysis was performed, with all participants included in the analysis according to original group allocation, and follow-up was maximized regardless of program attendance"

Comment: states ITT but the total number did not match 
Golley 2007 (Continued)

\begin{tabular}{l|l|l} 
Selective reporting (reporting bias) & High risk & $\begin{array}{l}\text { Comment: health-related quality of life and } \\
\text { satisfaction stated as outcomes but not re- } \\
\text { ported }\end{array}$ \\
\hline Other bias & Unclear risk & Comment: not enough detail to judge
\end{tabular}

Golan 2006

Methods

Parallel randomised controlled trial

Randomisation ratio: not reported

Superiority design

Participants

Inclusion criteria: children $>20 \%$ overweight (BMI for age and sex $>85$ th percentile) . Parents agreed to attend programme meetings. No current participation of any family member in a weight-loss programme. No restriction regarding participation in a physical activity programme for children and parents. No diagnosis of psychiatric or major endocrine pathology

Exclusion criteria: current participation of any family member in a weight-loss programme; restriction regarding participation in a physical activity programme for children and parents; diagnosis of psychiatric or major endocrine pathology

Diagnostic criteria: BMI for age and sex $>85$ th percentile

Interventions

Number of study centres: 1

Treatment before study: none

Titration period: none

Description of interventions:

Parent-only group:

$16 \times 1$ hour sessions at the following intervals: weeks 1-10 (10 sessions); bi-weekly - weeks 11-18 (4 sessions); monthly - weeks 19-26 (2 sessions). Nurturing the child emotionally. Problem solving. The first 3 sessions focused on nutrition education and parental modelling. In the next 2 sessions, the use of an authoritative feeding style was discussed. Sessions 6 and 7 focused on eating and activity behaviour modification, reinforcing means to influence a child's food preferences, as well as employing behaviour modification. Sessions 8 and 9 focused on problem solving while implementing the change in the home. Sessions 10 and 11 dealt with cognitive restructuring and media management. Session 12 focused on coping with resistance. In the remaining 4 sessions, groups discussed their successes and difficulties, as well as recommendations on how to work around constraints imposed on parents in order to promote a healthy lifestyle for all family members

Physical activity goals of 4 hours per week, and decrease in sedentary behaviours (to $<3$ hours/day)

Theoretical basis: parents as the exclusive agent of change. Authoritative feeding style. Nurturing. Parental modelling. Behaviour change. Based on previous work

Structured 12-session programme (unclear if a manual). A clinical dietician, supervised by a family therapist, administered the programme. Training of dietician not reported Parent and children group:

Similar in content; however, adapted to fit the children included

Parent-only interventions for childhood overweight or obesity in children aged 5 to II years (Review) 


\section{Golan 2006 (Continued)}

\begin{tabular}{l|l} 
Outcomes & $\begin{array}{l}\text { Outcomes reported in abstract of publication: \% overweight at end of programme } \\
(6 \text { months) and 1-year follow-up. Food stimuli in the home (from Family Eating and } \\
\text { Activity questionnaire). Parents' weight }\end{array}$ \\
\hline Study details & $\begin{array}{l}\text { Run-in period: none } \\
\text { Study terminated before regular end (for benefit/because of adverse events): no }\end{array}$ \\
\hline Publication details & $\begin{array}{l}\text { Language of publication: English } \\
\text { Funding not stated } \\
\text { Publication status: peer-reviewed journal }\end{array}$ \\
\hline
\end{tabular}

Stated aim for study

Quote from publication: “The question remains, which is better: parents only or parent and child treatment? The present study extends this knowledge by comparing targeting parents and child versus parents alone, to address the question: Do the children need to be involved at all?...”

Notes

Risk of bias

\begin{tabular}{|c|c|c|}
\hline Bias & Authors' judgement & Support for judgement \\
\hline $\begin{array}{l}\text { Random sequence generation (selection } \\
\text { bias) }\end{array}$ & Unclear risk & $\begin{array}{l}\text { Comment: randomisation stratified by } \\
\text { age groups: participants divided into age } \\
\text { groups: } 6-7 \text { years, } 8-9 \text { years, } 10-11 \text { years, } \\
\text { then randomised. No details of randomisa- } \\
\text { tion schedule }\end{array}$ \\
\hline Allocation concealment (selection bias) & Low risk & $\begin{array}{l}\text { Quote from publication: "The process was } \\
\text { carried out by using two concealed opaque } \\
\text { envelopes indicating group } 1 \text {, namely par- } \\
\text { ents-only, or group 2, parents and children" }\end{array}$ \\
\hline $\begin{array}{l}\text { Blinding of participants and personnel } \\
\text { (performance bias) } \\
\text { Subjective outcomes }\end{array}$ & Unclear risk & Comment: no details provided \\
\hline
\end{tabular}

Blinding of participants and personnel Unclear risk

Comment: no details provided

(performance bias)

Objective outcomes

Blinding of outcome assessment (detection Low risk bias)

Subjective outcomes

Blinding of outcome assessment (detection Low risk bias)

Objective outcome
Quote from publication: "The data were gathered by an MSc [Master of Science] student who was blinded to the treatment allocation"

Quote from publication: "The data were gathered by an MSc student who was

Copyright (c) 2015 The Cochrane Collaboration. Published by John Wiley \& Sons, Ltd. 
Incomplete outcome data (attrition bias) Low risk Subjective outcomes
Comment: ITT analysis where the missing values were replaced with baseline values. Reasonable attendance in both arms and numbers and reasons for drop-outs given

Comment: ITT analysis where the missing values were replaced with baseline values. Reasonable attendance in both arms and numbers and reasons for drop-outs given

Comment: all outcomes reported as stated but not enough information to judge

Comment: although power calculations given, 12 participants in each arm seems a small number from the power calculation

Aragona 1975

Methods

Randomisation ratio: superiority
Superiority design: $1: 1: 1$

Participants

Interventions programme reduction programme

Diagnostic criteria: not stated
Inclusion criteria: overweight girls aged 5-10 years. Children considered overweight if both their physician and parents recommended that they participate in the weight-loss

Exclusion criteria: undergoing psychotherapy, drug therapy or involved in a weight-

\section{Number of study centres: assume 1 \\ Treatment before study: none \\ Titration period: none \\ Intervention description:}

Response-cost plus reinforcement:

12-week treatment. Parents given daily weight and calorie graphs, a calorie counter guide and an eating diary. Parents also given a weight reduction programme behavioural contract, instruction on daily exercise and an exercise programme (daily calisthenics that increased in difficulty over a 3 -week period, thereafter 30 minutes per day), nutritional information, instructions in stimulus control techniques, a book 'Living with Children' and information in reinforcement techniques, a daily reinforcement diary. At second baseline visit given a response-cost contract to return the following week with money for deposit and a weight loss goal of between 1 and 2 pounds per week. Deposits were on a sliding scale of income vs. number of dependents and ranged between USD 12-30. Money could be redeemed in 12 weekly instalments (25\% weekly for attendance, $25 \%$ for bringing completed graphs and charts to the meeting, 50\% if the child lost the agreed 
weight). Unearned money divided among the successful parents. Children weighed and then sent to a playroom. After the programme there was an 8-week no contract followup and following that a post follow-up check 31 weeks later

Response-cost:

Parents given the same as the response-cost plus reinforcement group except did not receive the book, information on reinforcement techniques or the reinforcement diary Control:

Informed would be able to participate at a later date (waiting list control)

\begin{tabular}{ll} 
Outcomes & Outcomes reported in abstract of publication: weight change \\
\hline Study details & $\begin{array}{l}\text { Run-in period: none } \\
\text { Study terminated before regular end (for benefit/because of adverse events): no }\end{array}$ \\
\hline Publication details & $\begin{array}{l}\text { Language of publication: English } \\
\text { Non-commercial funding } \\
\text { Publication status: peer-reviewed publication }\end{array}$ \\
\hline
\end{tabular}

Stated aim for study

Quote from publication: “The present study treated with behavioural techniques overweight children 5 to 10 year of age"

Notes

There were 3 comparisons of relevance to this review: parent-only + reinforcement vs. parent-only; parent-only + reinforcement vs. control; parent-only vs. control

\section{Risk of bias}

\begin{tabular}{|c|c|c|}
\hline Bias & Authors' judgement & Support for judgement \\
\hline $\begin{array}{l}\text { Random sequence generation (selection } \\
\text { bias) }\end{array}$ & Unclear risk & $\begin{array}{l}\text { Quote from publication: "subjects were } \\
\text { randomly assigned to one of three groups" } \\
\text { Comment: no other details }\end{array}$ \\
\hline Allocation concealment (selection bias) & Unclear risk & Comment: no details \\
\hline $\begin{array}{l}\text { Blinding of participants and personnel } \\
\text { (performance bias) } \\
\text { Objective outcomes }\end{array}$ & Unclear risk & Comment: no details \\
\hline $\begin{array}{l}\text { Blinding of outcome assessment (detection } \\
\text { bias) } \\
\text { Objective outcome }\end{array}$ & Unclear risk & Comment: no details \\
\hline $\begin{array}{l}\text { Incomplete outcome data (attrition bias) } \\
\text { Objective outcomes }\end{array}$ & Low risk & $\begin{array}{l}\text { Comment: numbers and reasons for drop- } \\
\text { outs provided, differential drop-out be- } \\
\text { tween groups (small numbers) }\end{array}$ \\
\hline Selective reporting (reporting bias) & Unclear risk & $\begin{array}{l}\text { Comment: not enough information to } \\
\text { judge }\end{array}$ \\
\hline
\end{tabular}

Parent-only interventions for childhood overweight or obesity in children aged 5 to II years (Review) 
Aragona 1975 (Continued)

Other bias $\quad$ Unclear risk

Comment: not enough information to judge

Note: where the judgement is 'unclear' and the description is blank, the study did not report that particular outcome.

“-” denotes not reported.

BMI: body mass index; CBT: cognitive behavioural therapy; ITT: intention-to-treat; NOURISH: Nourishing Our Understanding of

Role modelling to Improve Support and Health; TV: television.

Characteristics of excluded studies [ordered by study ID]

\begin{tabular}{|c|c|}
\hline Study & Reason for exclusion \\
\hline Berry 2007 & Intervention not parent-only \\
\hline Biotnott 2009 & Duration of intervention/follow-up $<6$ months \\
\hline Bohlin 2012 & Intervention not parent-only \\
\hline De Bock 2013 & Intervention not parent-only \\
\hline Dewes 2014 & Intervention not parent-only \\
\hline Hendrie 2011 & Intervention not parent-only \\
\hline Hystad 2013 & Intervention not parent-only \\
\hline John 2009 & Duration of intervention/follow-up $<6$ months \\
\hline Lawson 2015 & Intervention not parent-only \\
\hline Le Gross 2006 & Duration of intervention/follow-up $<6$ months \\
\hline NHLBI 2008 & Primary prevention study \\
\hline Parra-Medina 2015 & Intervention not parent-only \\
\hline Shelton 2007 & Duration of intervention/follow-up $<6$ months \\
\hline Steele 2014 & Intervention not parent-only \\
\hline Volkenant 2011 & Duration of intervention/follow-up $<6$ months \\
\hline
\end{tabular}


(Continued)

Warschburger 2013 Intervention not parent-only

Characteristics of studies awaiting assessment [ordered by study ID]

Geronilla 1981

\begin{tabular}{ll}
\hline Methods & No details \\
\hline Participants & Obese children and adolescents \\
\hline Interventions & No details \\
\hline Outcomes & No details \\
\hline Study identifier & Dissertation abstract: $1982-72486-001$ \\
\hline Official title & A study of weight control in paediatric obesity using mothers as behavior modifiers \\
\hline Stated purpose of study & No details \\
\hline Notes & We were unable to access the full publication of this study \\
\hline
\end{tabular}

Gillick 1975

\begin{tabular}{ll}
\hline Methods & No details \\
\hline Participants & Mothers and their 6- to 12-year-old obese children \\
\hline Interventions & No details \\
\hline Outcomes & No details \\
\hline Study identifier & Dissertation abstract: $1977-13293-001$ \\
\hline Official title & Training parents as therapists in the treatment of juvenile obesity \\
\hline Stated purpose of study & No details \\
\hline Notes & We were unable to access the full publication of this study \\
\hline
\end{tabular}




$\begin{array}{ll}\text { Methods } & \text { Parallel randomised controlled trial } \\ \text { Randomisation ratio: not reported } \\ \text { Superiority design }\end{array}$

Participants Inclusion criteria: aged 6-11 years; weight $>20 \%$ over expected weight for age, height and gender; no history of psychiatric contact for children; and both parents living at home and parental agreement to meet all requirements of the study (check-ups, questionnaire, group sessions)

Exclusion criteria: the main reasons for exclusion were the children's reluctance to undergo blood sampling and the parents' denial of their children being obese or needing treatment

Diagnostic criteria: weight $>20 \%$ over expected weight for age, height and gender

\begin{tabular}{|c|c|}
\hline Interventions & $\begin{array}{l}\text { Number of study centres: } 1 \\
\text { Treatment before study: none } \\
\text { Titration period: none } \\
\text { Description of interventions } \\
\text { Parent-only: } \\
14 \text { x } 1 \text {-hour group sessions, conducted by clinical dietician, attended only by parents (delivered as } 2 \text { groups } \\
\text { of } 15 \text { parents). } 4 \text { sessions - weekly; } 4 \text { sessions - bi-weekly; } 6 \text { sessions - every } 6 \text { weeks. Also, } 5 \text { x } 15 \text { minute } \\
\text { individual sessions for whole family, during last } 6 \text { group sessions } \\
\text { Apply behavioural modifications (implement lifestyle change); practice parenting skills (overlap with nu- } \\
\text { trition advice as well). All instructions were oriented to the family system. At the sessions, the parents were } \\
\text { taught to alter the family sedentary lifestyle, provide a prudent diet (reduction of total and saturated fats, } \\
\text { increase of mono-unsaturated fatty acids), decrease the family's exposure to food stimuli, apply behavioural } \\
\text { modifications and practise relevant parenting skills. } \\
\text { Other topics discussed were limits of responsibilities, parental modelling, cognitive restructuring and coping } \\
\text { with resistance } \\
\text { Parent's role was to control the quality and pattern of the food environment, but not restrict the amount } \\
\text { of food eaten } \\
\text { Training of staff not reported, assumed delivered by a dietician } \\
\text { Control intervention: } \\
\text { Child prescribed calorie-controlled diet. Children divided into } 2 \text { subgroups. } 30 \text { x 1-hour group sessions, } \\
\text { by clinical dietician; } 8 \text { sessions - weekly; } 22 \text { session - biweekly } \\
\text { Children taught techniques how to follow a prudent diet, restrict energy intake, increase exercise, control } \\
\text { food stimuli, techniques in self monitoring, practise problem solving and cognitive restructuring, and make } \\
\text { use of social support. Individual counselling was offered when a child missed the group session or needed } \\
\text { extra support }\end{array}$ \\
\hline
\end{tabular}

Outcomes

Outcomes reported in abstract of publication: drop-out; mean reduction in percentage overweight; exposure to food stimuli/changes in eating habits

$\begin{array}{ll}\text { Study identifier } & \begin{array}{l}\text { Run-in period: none } \\ \text { Study terminated before regular end (for benefit/because of adverse events): no }\end{array}\end{array}$

Official title Language of publication: English

Non-commercial funding

Publication status: peer-reviewed journal

Parent-only interventions for childhood overweight or obesity in children aged 5 to I I years (Review) 


\section{Golan 1998 (Continued)}

Stated purpose of study Quote from publication: “This study examined the reduction in overweight and changes in eating-related behaviours in obese children treated with a family-based approach, in which the parents were the exclusive agents of change. Results were compared to the conventional approach in which children are responsible for their own weight loss"

\section{Characteristics of ongoing studies [ordered by study ID]}

\section{Ball 2012}

\begin{tabular}{ll}
\hline Trial name or title & Acronym: PAC \\
\hline Methods & $\begin{array}{l}\text { Type of study: efficacy study } \\
\text { Allocation: randomised } \\
\text { Intervention model: parallel assignment } \\
\text { Masking: single blind (participants) } \\
\text { Primary purpose: weight loss intervention }\end{array}$ \\
\hline $\begin{array}{l}\text { Condition: parents of overweight children } \\
\text { Enrolment: estimated } 90 \\
\text { Inclusion criteria: families are eligible for this study if children are aged 8-12 years, children present with } \\
\text { an age- and sex-specific BMI } \geq 85 \text { th percentile, at least } 1 \text { parent agrees to attend weekly PAC sessions for } \\
\text { 16 weeks and children and parents are fluent in English (verbal and written) } \\
\text { Exclusion criteria: not stated }\end{array}$ \\
$\begin{array}{l}\text { Intervention(s): Parents as Agents of Change (PAC) intervention (includes cognitive behavioural therapy) } \\
\text { Comparator(s): psycho-educational-based intervention } \\
\text { Both intervention arms in the trial are the same in frequency of contact (16 sessions), content (identical } \\
\text { information is delivered), mode (group format), duration (60-90 minutes per session), intervention goals } \\
\text { (related to nutrition and physical activity) and the number of group leaders (2 per group). The intervention } \\
\text { arms differ in how information is conveyed to parents, and how parents work towards attempting, achieving } \\
\text { and maintaining healthy cognitive and behavioural changes }\end{array}$ \\
\hline $\begin{array}{l}\text { Interventions } \\
\text { and }\end{array}$ \\
\hline
\end{tabular}

Outcomes

Primary outcome(s): BMI z score

Secondary outcome(s): lifestyle behaviours, nutrition and physical activity behaviours; Parental Stress Index (PSI); blood pressure; fasting glucose; fasting insulin; HDL cholesterol; LDL cholesterol; total cholesterol; triglycerides (child only); Family Adaptability and Cohesion Scale-IV (FACES-IV)

Other outcome(s): as above

$\begin{array}{ll}\text { Starting date } & \begin{array}{l}\text { Study start date: September } 2010 \\ \text { Study completion date: January } 2014\end{array}\end{array}$

Contact information

Responsible party/principal investigator: Geoff Ball (gdball@ualberta.ca), University of Alberta, Canada

Study identifier

NCT01267097

Parent-only interventions for childhood overweight or obesity in children aged 5 to II years (Review) 
Ball 2012 (Continued)

Official title Parents as Agents of Change (PAC) in paediatric weight management

Stated purpose of study Quote: "We hypothesize that children with obesity whose parents complete a CBT-based PAC intervention will achieve greater reductions in adiposity and improvements in cardiometabolic risk factors, lifestyle behaviours, and psychosocial outcomes than children whose parents complete a psycho-education-based PAC intervention (PEP)"

Notes

\section{Dalton 2011}

Trial name or title Acronym: PLAN

Methods $\quad$ Type of study: efficacy study

Allocation: randomised

Intervention model: cluster trial

Masking: none

Primary purpose: weight loss intervention

Participants Condition: parents of overweight children

Enrolment: estimated 80

Inclusion criteria: $\mathrm{BMI} \geq$ the 85 th percentile during the recruitment period, and 1 parent or other primary carer agrees to participate in the study, including individual visits and group sessions as well as telephone follow-ups. Only one child per family will be included in the study

Exclusion criteria: current child or parent/primary carer participation in a weight management programme; presence of a diagnosed psychiatric/psychological disorder in the child or parent/primary carer (e.g. attention deficit hyperactivity disorder, autism, eating disorder); presence of an underlying medical condition affecting weight status (e.g. hypothyroidism, Cushing's syndrome, chronic steroid use); current dietary or physical activity restrictions (e.g. such as in children with diabetes or orthopaedic problems including slipped capital femoral epiphysis); and parents/primary carers do not have telephone accessibility. Parents/primary carers will also have to understand and speak English

Interventions

Intervention(s): parent-mediated childhood overweight intervention (PLAN) for healthy living and the parent handbook described below. 2 individual visits with clinic provider, 4 clinic-based group sessions moderated by a trained clinic provider and 4 follow-up telephone calls from the Project Co-ordinator or research staff

Comparator(s): parents will receive "Families Finding the Balance: A Parent Handbook", a health education handbook adopted by NIH We Can! during the baseline assessment

Outcomes Primary outcome(s): BMI z score

Secondary outcome(s): child's and family's eating and physical activity behaviours and the child's healthrelated quality of life

Other outcome(s): covariates; healthcare provider perceptions of treatment of child overweight and obesity. Parents and healthcare providers will also complete surveys and focus groups, respectively, on the acceptability and feasibility of this approach including provider perceptions of training

Starting date

Study start date: not reported

Study completion date: not reported

Parent-only interventions for childhood overweight or obesity in children aged 5 to I I years (Review)

Copyright @ 2015 The Cochrane Collaboration. Published by John Wiley \& Sons, Ltd. 


\section{Dalton 2011 (Continued)}

\begin{tabular}{ll} 
Contact information & $\begin{array}{l}\text { Responsible party/principal investigator: Karen E Schetzina, East Tennessee State University, Johnson } \\
\text { City, Tennessee, USA }\end{array}$ \\
\hline Study identifier & NCT number: NCT01729910 \\
\hline Official title & $\begin{array}{l}\text { Parent-Led Activity and Nutrition (PLAN) for Healthy Living (published protocol) } \\
\text { Primary Care Child Obesity Intervention Targeting Parents (trial document) }\end{array}$ \\
\hline Stated purpose of study & $\begin{array}{l}\text { Quote: "1) to establish a primary care based and parent-mediated childhood overweight intervention } \\
\text { program in the primary care setting, 2) to explore the efficacy of this intervention in promoting healthier } \\
\text { weight status and health behaviours of children, 3) to examine the acceptability and feasibility of the } \\
\text { approach among parents and primary care providers" }\end{array}$ \\
\hline Notes & \begin{tabular}{l}
\hline \\
\hline
\end{tabular}
\end{tabular}

Gerards 2012

\begin{tabular}{ll}
\hline Trial name or title & Acronym: GO4fit \\
\hline Methods & $\begin{array}{l}\text { Type of study: efficacy study } \\
\text { Allocation: randomised } \\
\text { Intervention model: parallel assignment } \\
\text { Masking: none } \\
\text { Primary purpose: weight loss intervention }\end{array}$ \\
\hline Participants & $\begin{array}{l}\text { Condition: parents of overweight children } \\
\text { Enrolment: estimated } 84 \text { child-parent triads } \\
\text { Inclusion criteria: parents of children are eligible for participation if their child is considered overweight } \\
\text { or obese, based on the BMI, using the international sex- and age-specific cut-off points proposed by Cole } \\
\text { et al } \\
\text { Exclusion criteria: none stated }\end{array}$ \\
\hline $\begin{array}{l}\text { Intervention(s): Lifestyle Triple P intervention for parents with active skills training methods based on self } \\
\text { regulation principles, to provide parents with new knowledge and skills. 14-week intervention, } 8 \text { weekly } \\
\text { 90-minute parental group sessions, followed by } 2 \text { weekly } 15-30 \text { minute telephone sessions, } 1 \text { further } 90- \\
\text { minute group session, } 2 \text { weekly } 15-\text { to } 30 \text {-minute telephone sessions, and a final } 90 \text {-minute group session } \\
\text { Comparator(s): receive } 2 \text { brochures }(1 \text { on healthy nutrition and physical activity, and } 1 \text { on positive } \\
\text { parenting), web-based tailored advice on setting a good example to their child, and suggestions for exercises } \\
\text { to increase active play at home }\end{array}$ \\
\hline
\end{tabular}

Outcomes Primary outcome(s): BMI z score, waist circumference, fat mass

Secondary outcome(s): children's dietary behaviour and physical activity level, parenting practices, parental feeding style, parenting styles, parental self efficacy, and body composition of family members (parents and siblings)

Other outcome(s): as above

Starting date

Study start date: December 2010

Study completion date: December 2012

Parent-only interventions for childhood overweight or obesity in children aged 5 to II years (Review) 
Gerards 2012 (Continued)

\begin{tabular}{l|l} 
Contact information & $\begin{array}{l}\text { Responsible party/principal investigator: Sanne Gerards (sanne.gerards@maastrichtuniversity.nl), Maas- } \\
\text { tricht University, The Netherlands }\end{array}$ \\
\hline Study identifier & The Netherlands National Trial Register NTR2555 \\
\hline Official title & $\begin{array}{l}\text { Lifestyle Triple P: a parenting intervention for childhood obesity (published protocol) } \\
\text { Effectiveness of Lifestyle Triple P: an intervention aimed at the prevention of excessive weight gain in 4- } \\
\text { till 8-year-old overweight children. - GO4fit (trial document) }\end{array}$
\end{tabular}

Stated purpose of study Quote: "The aim of the current randomised controlled trial is to assess the effectiveness of the Lifestyle Triple P intervention when applied to Dutch parents of overweight and obese children aged 4-8 years"

Notes

Janicke 2011

\begin{tabular}{ll}
\hline Trial name or title & Acronym: E-FLIP for Kids \\
\hline Methods & $\begin{array}{l}\text { Type of study: efficacy study } \\
\text { Allocation: randomised } \\
\text { Intervention model: parallel assignment } \\
\text { Masking: not reported } \\
\text { Primary purpose: treatment }\end{array}$ \\
\hline Participants & $\begin{array}{l}\text { Condition: overweight and obese } \\
\text { Enrolment: estimated } 240\end{array}$ \\
\hline $\begin{array}{l}\text { Inclusion criteria: aged } 8-12 \text { years, BMI } \geq 85 \text { th percentile for age and gender, living within the same } \\
\text { dwelling in a rural county. Participating parents or legal guardians must be age } \leq 75 \text { years } \\
\text { Exclusion criteria: participating parent has a dietary or exercise restriction, or a medical condition that } \\
\text { contraindicates mild energy restriction or moderate physical activity. Children or participating parents on } \\
\text { antipsychotic agents, systemic corticosteroids, antibiotics for HIV or tuberculosis, chemotherapeutic drugs } \\
\text { or who are currently using prescription weight-loss drugs, child has a resting blood pressure } \geq 140 / 90 \\
\text { mmHg, not engaged in another weight control programme, exhibit conditions or behaviours that are likely } \\
\text { to affect their participation in the trial, such as being unwilling or unable to give informed consent, parent } \\
\text { (s) or legal guardian(s) unable to read English at approximately the 5th grade level, unwilling to accept } \\
\text { random assignment, unable to travel to the extension office for intervention sessions, or likely to move out } \\
\text { of the county within the next } 24 \text { months }\end{array}$ \\
\hline
\end{tabular}

Interventions

\section{Interventions:}

1. General intervention + parent-only intervention: weekly group sessions for the first 8 weeks, then 4 biweekly sessions over the next 8 weeks for a total of 12 sessions across 16 weeks. Contact will then fade to 1 group session per month for months 5-12, with the exception of month 9, during which participants will attend 2 group sessions. Each session will last 90 minutes. Focus on diet, physical activity and behavioural components

2. General intervention + family-based behavioural intervention: as above but involves children and parents who will meet in simultaneous, but separate, parent-child groups at each meeting

Comparator(s): 21 group meetings on the same schedule as the intervention arms. Each session will last 90

Parent-only interventions for childhood overweight or obesity in children aged 5 to I I years (Review) 
Janicke 2011 (Continued)

minutes. The families in the Health Education group will not receive training in behavioural self regulation strategies, such as goal setting and self monitoring. There will be no instruction on behavioural parenting strategies and parent

Primary outcome(s): BMI z score
Secondary outcome(s): child body fat, waist circumference, height, weight, dietary intake, physical activity,
blood lipids, blood glucose, parental measures, health-related quality of life, parenting skills, costs and cost
effectiveness

Starting date Study start date: July 2009

Study completion date: December 2014

Contact information Responsible party/principal investigator: David Janicke (djanicke@phhp.ufl.edu)

Study identifier NCT number: NCT01820338

Official title

The Extension Family Lifestyle Intervention Project (E-FLIP for Kids) (published protocol and trial document)

Stated purpose of study Quote: "assessing the effectiveness of two behavioral weight management interventions in an important and at-risk population, overweight and obese children and their parents in rural counties..."

Notes

NCT01197443

Trial name or title Acronym: PAAC

Methods Type of study: efficacy and cost effectiveness study

Allocation: randomised

Intervention model: parallel assignment

Masking: none

Primary purpose: weight loss intervention

Participants Condition: parents of overweight children

Enrolment: estimated 150

Inclusion criteria: overweight child $>95$ th percentile for age and gender; an overweight $(\mathrm{BMI}>25)$ parent willing to participate and attend all treatment meetings; eligible parent who can read at a minimum of an 8th grade level; family willing to commit to 5 months of treatment attendance and follow-up for 18 months post-treatment

Exclusion criteria: major child psychiatric disorder diagnoses; child diagnoses of a serious current physical disease (such as diabetes) for which physician supervision of diet and exercise prescription are needed (self report); family with restrictions on types of food, such as food allergies, religious or ethnic practices that limit the foods available in the home; child with physical difficulties that limit the ability to exercise; child with an active eating disorder (based on Eating Disorder Examination interview); families where children or parents are involved in swimming or weight training more than 5 hours per week; major parent psychiatric disorder

Parent-only interventions for childhood overweight or obesity in children aged 5 to I I years (Review) 


\section{NCT01197443 (Continued)}

\begin{tabular}{|c|c|}
\hline Interventions & $\begin{array}{l}\text { Intervention(s): behavioural parent-only intervention } \\
\text { Comparator(s): behavioural parent-child intervention } \\
\text { The treatment length is set for } 12 \text { weekly meetings and bi-monthly meetings during months } 4 \text { and } 5 \text {. Each } \\
\text { group session will be } 60 \text { minutes including weigh-ins }\end{array}$ \\
\hline Outcomes & $\begin{array}{l}\text { Primary outcome(s): weight; BMI for age percentile; BMI z score } \\
\text { Secondary outcome(s): cost effectiveness, dietary quality, exercise behaviour, quality of life, psychosocial } \\
\text { measures, parenting adherence, parenting style, parent weight loss, compliance and changes in household } \\
\text { food environment }\end{array}$ \\
\hline Starting date & $\begin{array}{l}\text { Study start date: November } 2010 \\
\text { Study completion date: July } 2015\end{array}$ \\
\hline Contact information & $\begin{array}{l}\text { Responsible party/principal investigator: Kerri Boutelle (kboutelle@ucsd.edu), University of California, } \\
\text { San Diego, USA }\end{array}$ \\
\hline Study identifier & NCT number: NCT01197443 \\
\hline Official title & Parents as the Agent of Change for Childhood Obesity (PAAC) \\
\hline Stated purpose of study & $\begin{array}{l}\text { Quote: "To evaluate the efficacy of parent only treatment versus parent }+ \text { child treatment in the body } \\
\text { weight of the target child. To evaluate the cost effectiveness compared to current gold standard treatment } \\
\text { of parent and child dual education" }\end{array}$ \\
\hline Notes & \\
\hline
\end{tabular}

\section{NCT01546727}

Trial name or title Acronym: Behavioral Treatment for Obese Preschoolers (LAUNCH)

\begin{tabular}{ll}
\hline Methods & $\begin{array}{l}\text { Type of study: efficacy } \\
\text { Allocation: randomised } \\
\text { Intervention model: factorial assignment } \\
\text { Masking: double blind } \\
\text { Primary purpose: treatment }\end{array}$ \\
\hline Participants & $\begin{array}{l}\text { Condition: obesity } \\
\text { Enrolment: estimated } 168\end{array}$ \\
Inclusion criteria: children aged 2 years 0 months to 5 years 11 months; BMI percentile $\geq 95$ th percentile \\
for age and gender, but no more than $100 \%$ above the median BMI for age and gender; English-speaking; \\
Live within 50 miles of Cincinnati Children's Hospital Medical Center (CCHMC); medical clearance from \\
the child's paediatrician to participate \\
Exclusion criteria: medical conditions known to promote obesity (e.g. Prader-Willi syndrome, Cushing's \\
syndrome); already involved with another weight control programme; taking weight-affecting medications \\
(e.g. steroids); a disability or illness that would preclude them from engaging in at least moderate intensity \\
physical activity; developmental disability
\end{tabular}

Parent-only interventions for childhood overweight or obesity in children aged 5 to I I years (Review) 


\section{NCT01546727 (Continued)}

\begin{tabular}{|c|c|}
\hline Interventions & $\begin{array}{l}\text { Intervention(s): } \\
\text { 1. Behavioural family intervention. } 3 \text { months of weekly treatment delivered via alternating group-based } \\
\text { clinic visits and individual home visits followed by } 3 \text { months of every other week treatment alternating } \\
\text { between clinic and home } \\
\text { 2. Behavioural: motivational interviewing - } 4 \text { in-person visits spaced at first visit, month } 3 \text { and month } 5 \text {. } \\
\text { Weekly telephone calls during the first } 3 \text { months and every other week during months 4-6 } \\
\text { Comparator(s): standard care }\end{array}$ \\
\hline Outcomes & $\begin{array}{l}\text { Primary outcomes: BMI z score change } \\
\text { Secondary outcome(s): BMI z score; caloric intake; physical activity; home environment; parent caloric } \\
\text { intake; parent physical activity; parent and child eating behaviours; health-related quality of life }\end{array}$ \\
\hline Starting date & $\begin{array}{l}\text { Study start date: March } 2012 \\
\text { Study completion date: November } 2016\end{array}$ \\
\hline Contact information & $\begin{array}{l}\text { Responsible party/principal investigator: Lori J Stark, Children's Hospital Medical Center, Cincinnati, } \\
\text { USA }\end{array}$ \\
\hline Study identifier & NCT number: NCT01546727 \\
\hline Official title & Clinic and Home Family Based Behavioral Treatment for Obese Preschoolers \\
\hline Stated purpose of study & $\begin{array}{l}\text { Quote: "to test a clinic and home family behavioral intervention (LAUNCH) against 1) motivational } \\
\text { interviewing (attention control; MI) and 2) standard of care (true standard of care control" }\end{array}$ \\
\hline Notes & \\
\hline
\end{tabular}

\section{NCT01552642}

\begin{tabular}{ll}
\hline Trial name or title & Acronym: none \\
\hline Methods & $\begin{array}{l}\text { Type of study: efficacy } \\
\text { Allocation: randomised } \\
\text { Intervention model: parallel } \\
\text { Masking: open label } \\
\text { Primary purpose: treatment }\end{array}$ \\
\hline Participants & $\begin{array}{l}\text { Condition: obesity, overweight } \\
\text { Enrolment: estimated } 156 \\
\text { Inclusion criteria: } 1 \text { parent of a 3- to 6-year old child with a BMI ? } 5 \text { 85th percentile, Internet access, } \\
\text { English speaking } \\
\text { Exclusion criteria: children with a developmental disorder, children with a chronic underlying disease that } \\
\text { may contribute to obesity, children taking medication that may interfere with a healthy weight }\end{array}$ \\
\hline Interventions & $\begin{array}{l}\text { Intervention: parenting behavioural intervention: } 6 \text { weekly face-to-face group (10-12 parents) meetings } \\
\text { and access to a website. Contents include authoritative parenting, using the food plan "Go, Slow, and } \\
\text { Whoa", increasing physical activity and behaviour change strategies. Parenting skills will be discussed at } \\
\text { every session. Website has information and links about nutrition and physical activity, an interactive group }\end{array}$ \\
\hline
\end{tabular}


NCT01552642 (Continued)

session and an ask the expert facility

Comparator: no intervention

Primary outcome: feasibility
Secondary outcomes: BMI z scores, healthy behaviour changes, parenting skills
Other outcome(s): -

Starting date Study start date: February 2013

Study completion date: August 2015

Contact information Responsible party/principal investigator: Ellen R Wald, University of Wisconsin, Madison, USA

Study identifier $\quad$ NCT number: NCT01552642

Official title An Interactive Web-based Intervention to Achieve Healthy Weight in Young Children

Stated purpose of study Quote: "to develop and implement an effective intervention program designed to prevent and treat obesity in young children"

Notes

\section{NCT01792531}

\begin{tabular}{ll} 
Trial name or title & Acronym: More and Less study $(\mathrm{M}+\mathrm{L})$ \\
\hline Methods & $\begin{array}{l}\text { Type of study: efficacy } \\
\text { Allocation: randomised } \\
\text { Intervention model: parallel } \\
\text { Masking: open label } \\
\text { Primary purpose: treatment }\end{array}$ \\
\hline Participants & $\begin{array}{l}\text { Condition: obesity } \\
\text { Enrolment: estimated } 180 \\
\text { Inclusion criteria: age } 4-6 \text { years old; obesity as defined by international cut-offs } \\
\text { Exclusion criteria: weight affecting diseases }\end{array}$ \\
\hline Interventions & $\begin{array}{l}\text { Intervention(s): parent training group with } 2 \text { subgroups, 12-week only vs. bolster sessions at } 8 \text {-week } \\
\text { intervals over } 1 \text { year } \\
\text { Focus on how to use positive parenting practices instead of ineffective practices. } 12 \text { sessions (1.5 hours } \\
\text { per week), introduction to effective parenting practices, discussion and practice using role play and home } \\
\text { practice assignments. Tailored to focus on changes in the home environment, mostly related to child food } \\
\text { habits and physical activity } \\
\text { Comparator(s): standard treatment with focus on lifestyle, provided by local paediatricians in outpatient } \\
\text { paediatric departments and will be based on lifestyle modifications, as recommended in the action plan for } \\
\text { Stockholm County }\end{array}$
\end{tabular}




\section{NCT01792531 (Continued)}

Primary outcome(s): BMI change at 1 year
Secondary outcome(s): changes in: parenting practices; child's dietary intake and behaviour; child's phys-
ical activity; family functioning; child's metabolic health; parental functioning; waist circumference; child's
functioning, socioeconomic status
Other outcome(s): -

Starting date

Study start date: January 2013

Study completion date: December 2017

Contact information Responsible party/principal investigator: Paulina Nowicka, Karolinska Institutet, Sweden

Study identifier $\quad$ NCT number: NCT01792531

Official title

The More \& Less Study: A Trial Testing Different Treatment Approaches to Obesity in Preschoolers (M\& L)

Stated purpose of study Quote: "to evaluate the effectiveness of early treatment of childhood obesity"

Notes

NCT02373670

\begin{tabular}{ll} 
Trial name or title & Acronym: none \\
\hline Methods & $\begin{array}{l}\text { Type of study: interventional } \\
\text { Allocation: randomised } \\
\text { Intervention model: parallel } \\
\text { Masking: open label } \\
\text { Primary purpose: treatment }\end{array}$ \\
\hline Participants & $\begin{array}{l}\text { Condition: obesity } \\
\text { Enrolment: estimated } 60 \\
\text { Inclusion criteria: aged 2-5 years, BMI z score }>2 \\
\text { Exclusion criteria: medical conditions or receiving medications affecting weight }\end{array}$
\end{tabular}

Interventions Intervention: parent mentors using positive deviance findings to promote healthy behaviours. Using locally derived positive deviance findings to inform a behavioural intervention

Comparator: education, community health workers providing health education to promote healthy behaviour. Using standardised healthy behaviour education (EatPlayGrow curriculum from NHLBI)

Outcomes

Primary outcome: BMI z score

Secondary outcome(s): not reported

Other outcome(s): not reported

Starting date

Study start date: January 2015

Study completion date: June 2015

Parent-only interventions for childhood overweight or obesity in children aged 5 to I I years (Review) 


\section{NCT02373670 (Continued)}

\begin{tabular}{ll} 
Contact information & $\begin{array}{l}\text { Responsible party/principal investigator: The University of Texas Health Science Center at San Antonio, } \\
\text { USA }\end{array}$ \\
\hline Study identifier & NCT number: NCT02373670 \\
\hline Official title & Parent Mentors Using Positive Deviance in Childhood Obesity \\
\hline Stated purpose of study & $\begin{array}{l}\text { Quote: “A feasibility study randomising participants (parents of children age 2-5 years old) to receive either } \\
\text { education or a parent mentor with the aim of improving health behaviours and improving their body mass } \\
\text { index z-score" }\end{array}$ \\
\hline Notes & \begin{tabular}{l} 
\\
\hline
\end{tabular}
\end{tabular}

Önnerfält 2012

\begin{tabular}{|c|c|}
\hline Trial name or title & Acronym: LOOPS \\
\hline Methods & $\begin{array}{l}\text { Type of study:interventional } \\
\text { Allocation: randomised } \\
\text { Intervention model: parallel } \\
\text { Masking: open label } \\
\text { Primary purpose: treatment }\end{array}$ \\
\hline Participants & $\begin{array}{l}\text { Condition: overweight, obese } \\
\text { Enrolment: estimated } 240 \text { ( } 160 \text { overweight, } 80 \text { obese) } \\
\text { Inclusion criteria: } 4 \text { - to } 6 \text {-year old children with overweight and obesity } \\
\text { Exclusion criteria: do not understand written and spoken Swedish well enough to participate in group } \\
\text { activities }\end{array}$ \\
\hline Interventions & $\begin{array}{l}\text { Intervention(s): } \\
\text { All start with a 2-hour lecture with general facts about overweight in children (GFO), performed by health } \\
\text { professionals. Also access to a website, Healthy Children (HC), with general information about diet and } \\
\text { exercise recommendations } \\
\text { Obese children randomised to either: } \\
\text { - Better balance every day (BBE) - parenthood and lifestyle, run by a clinical psychologist, parents } \\
\text { attend } 6 \text { x 2-hour sessions over } 12 \text { months } \\
\text { - Lighter Living (LiLi), run by an occupational therapist, based on the theory that alterations } \\
\text { in the parents'-everyday life will induce changes that will gradually lead to a normalisation of their children's } \\
\text { weight. Groups meet for } 13 \times 2 \text {-hour sessions over } 12 \text { months } \\
\text { Overweight children randomised to } 1 \text { of } 3 \text { groups } \\
\text { - BBE as above } \\
\text { - Website only: information in health food and physical activity, based on national guidelines and } \\
\text { recommendations for pre-school children whether overweight or not; parents can ask questions to } \\
\text { paediatrician, a dietician, a psychologist or an occupational therapist } \\
\text { - Control (general lecture only) } \\
\text { Parents are invited to attend group meetings with the general purpose of supporting the parents to accom- } \\
\text { plish preferred lifestyle changes, both in the short and long term } \\
\text { Comparator(s): as above }\end{array}$ \\
\hline
\end{tabular}

Parent-only interventions for childhood overweight or obesity in children aged 5 to II years (Review) 
Önnerfält 2012 (Continued)

\begin{tabular}{|c|c|}
\hline Outcomes & $\begin{array}{l}\text { Primary outcome(s): change in BMI z score } \\
\text { Secondary outcome(s): dietary and exercise patterns, waist circumference, insulin resistance, dietary hor- } \\
\text { mones, fecal micro-flora } \\
\text { Other outcome(s): parent change in BMI, perception of their own health, parent stress, child feeding and } \\
\text { exercise habits }\end{array}$ \\
\hline Starting date & $\begin{array}{l}\text { Study start date: August } 2008 \\
\text { Study completion date: November } 2015\end{array}$ \\
\hline Contact information & $\begin{array}{l}\text { Responsible party/principal investigator: Kristina Thorngren-Jerneck (Kristina.Thorngren-Jer- } \\
\text { neck@med.lu.se), Lund University Children's hospital, Sweden }\end{array}$ \\
\hline Study identifier & NCT number: NCT00916318 \\
\hline Official title & $\begin{array}{l}\text { Overweight and Obesity in Preschool Children, Prevalence and Prevention - Family Based Health Inter- } \\
\text { ventions for Child Health (trial document) } \\
\text { LOOPS - Lund Overweight and Obesity Preschool Study (published protocol) }\end{array}$ \\
\hline Stated purpose of study & $\begin{array}{l}\text { Quote: "to evaluate if a family-based intervention, targeting parents of preschool children with overweight } \\
\text { and obesity, has a long-term positive effect on weight development of the children" }\end{array}$ \\
\hline Notes & \\
\hline
\end{tabular}

BMI: body mass index; HDL: high-density lipoprotein; LDL: low-density lipoprotein. 
DATA ANDANALYSES

Comparison 1. Parent-only interventions versus parent-child interventions

\begin{tabular}{|c|c|c|c|c|}
\hline Outcome or subgroup title & $\begin{array}{l}\text { No. of } \\
\text { studies }\end{array}$ & $\begin{array}{c}\text { No. of } \\
\text { participants }\end{array}$ & Statistical method & Effect size \\
\hline $\begin{array}{l}1 \text { BMI z score change post } \\
\text { intervention }\end{array}$ & 3 & 277 & Mean Difference (IV, Random, 95\% CI) & $-0.06[-0.13,0.02]$ \\
\hline $\begin{array}{l}\text { 1.1 Parent-only vs. } \\
\text { parent-child }\end{array}$ & 2 & 112 & Mean Difference (IV, Random, 95\% CI) & $-0.05[-0.13,0.04]$ \\
\hline $\begin{array}{l}\text { 1.2 Parent-only vs. } \\
\text { parent-child physical activity }\end{array}$ & 1 & 84 & Mean Difference (IV, Random, 95\% CI) & $-0.15[-0.26,-0.04]$ \\
\hline $\begin{array}{l}\text { 1.3 Parent-only vs. } \\
\text { parent-child physical activity + } \\
\text { diet }\end{array}$ & 1 & 81 & Mean Difference (IV, Random, 95\% CI) & $0.0[-0.11,0.11]$ \\
\hline $\begin{array}{l}2 \text { BMI z score change longest } \\
\text { follow-up }\end{array}$ & 3 & 267 & Mean Difference (IV, Random, 95\% CI) & $-0.04[-0.15,0.08]$ \\
\hline $\begin{array}{l}2.1 \text { Parent-only vs. } \\
\text { parent-child }\end{array}$ & 2 & 102 & Mean Difference (IV, Random, 95\% CI) & $0.06[-0.05,0.16]$ \\
\hline $\begin{array}{l}2.2 \text { Parent-only vs. } \\
\text { parent-child physical activity }\end{array}$ & 1 & 84 & Mean Difference (IV, Random, 95\% CI) & $-0.16[-0.36,0.04]$ \\
\hline $\begin{array}{l}2.3 \text { Parent-only vs. } \\
\text { parent-child physical activity + } \\
\text { diet }\end{array}$ & 1 & 81 & Mean Difference (IV, Random, 95\% CI) & $-0.11[-0.31,0.09]$ \\
\hline
\end{tabular}

Comparison 2. Parent-only interventions versus waiting list interventions

\begin{tabular}{|c|c|c|c|c|}
\hline Outcome or subgroup title & $\begin{array}{l}\text { No. of } \\
\text { studies }\end{array}$ & $\begin{array}{c}\text { No. of } \\
\text { participants }\end{array}$ & Statistical method & Effect size \\
\hline $\begin{array}{l}1 \text { BMI z score change post } \\
\text { intervention }\end{array}$ & 2 & 153 & Mean Difference (IV, Random, 95\% CI) & $-0.12[-0.21,-0.04]$ \\
\hline $\begin{array}{l}2 \text { BMI z score change longest } \\
\text { follow-up }\end{array}$ & 2 & 136 & Mean Difference (IV, Fixed, 95\% CI) & $-0.10[-0.19,-0.01]$ \\
\hline 2.1 Parent-only vs. waiting list & 2 & 92 & Mean Difference (IV, Fixed, 95\% CI) & $-0.11[-0.21,-0.01]$ \\
\hline $\begin{array}{l}\text { 2.2 Parent-only intensive } \\
\text { education vs. waiting list }\end{array}$ & 1 & 44 & Mean Difference (IV, Fixed, 95\% CI) & $-0.02[-0.29,0.25]$ \\
\hline $\begin{array}{l}3 \text { BMI percentile change post } \\
\text { intervention }\end{array}$ & 1 & & Mean Difference (IV, Fixed, 95\% CI) & Totals not selected \\
\hline $\begin{array}{l}4 \text { BMI percentile change longest } \\
\text { follow-up }\end{array}$ & 1 & & Mean Difference (IV, Fixed, 95\% CI) & Totals not selected \\
\hline 5 BMI change post intervention & 1 & & Mean Difference (IV, Fixed, 95\% CI) & Totals not selected \\
\hline $\begin{array}{l}5.1 \text { Parent-only reinforcement } \\
\text { vs. waiting list }\end{array}$ & 1 & & Mean Difference (IV, Fixed, 95\% CI) & $0.0[0.0,0.0]$ \\
\hline 5.2 Parent-only vs. waiting list & 1 & & Mean Difference (IV, Fixed, 95\% CI) & $0.0[0.0,0.0]$ \\
\hline $6 \mathrm{BMI}$ change longest follow-up & 1 & & Mean Difference (IV, Fixed, 95\% CI) & Totals not selected \\
\hline
\end{tabular}

Parent-only interventions for childhood overweight or obesity in children aged 5 to II years (Review)

Copyright @ 2015 The Cochrane Collaboration. Published by John Wiley \& Sons, Ltd. 


\section{Comparison 3. Parent-only interventions versus minimal contact interventions}

\begin{tabular}{|c|c|c|c|c|}
\hline Outcome or subgroup title & $\begin{array}{l}\text { No. of } \\
\text { studies }\end{array}$ & $\begin{array}{c}\text { No. of } \\
\text { participants }\end{array}$ & Statistical method & Effect size \\
\hline $\begin{array}{l}1 \text { BMI z score change post } \\
\text { intervention }\end{array}$ & 1 & 170 & Mean Difference (IV, Random, 95\% CI) & $-.00[-0.08,0.08]$ \\
\hline $\begin{array}{l}\text { 1.1 Parent-only IVR vs. } \\
\text { control }\end{array}$ & 1 & 87 & Mean Difference (IV, Random, 95\% CI) & $-0.02[-0.13,0.09]$ \\
\hline 1.2 Parent-only vs. control & 1 & 83 & Mean Difference (IV, Random, 95\% CI) & $0.02[-0.09,0.13]$ \\
\hline $\begin{array}{l}2 \text { BMI z score change longest } \\
\text { follow-up }\end{array}$ & 1 & 165 & Mean Difference (IV, Fixed, 95\% CI) & $0.01[-0.07,0.09]$ \\
\hline $\begin{array}{l}\text { 2.1 Parent-only interactive } \\
\text { voice response vs. control }\end{array}$ & 1 & 86 & Mean Difference (IV, Fixed, 95\% CI) & $-0.02[-0.13,0.09]$ \\
\hline 2.2 Parent-only vs. control & 1 & 79 & Mean Difference (IV, Fixed, 95\% CI) & $0.04[-0.07,0.15]$ \\
\hline $\begin{array}{l}3 \text { BMI percentile change post } \\
\text { intervention }\end{array}$ & 4 & & Mean Difference (IV, Random, 95\% CI) & Totals not selected \\
\hline $\begin{array}{l}\text { 3.1 Parent-only vs. minimal } \\
\text { contact control }\end{array}$ & 3 & & Mean Difference (IV, Random, 95\% CI) & $0.0[0.0,0.0]$ \\
\hline $\begin{array}{l}\text { 3.2 Parent motivational } \\
\text { interviewing vs. minimal } \\
\text { contact control }\end{array}$ & 1 & & Mean Difference (IV, Random, 95\% CI) & $0.0[0.0,0.0]$ \\
\hline $\begin{array}{l}\text { 3.3 Parent motivational } \\
\text { interviewing + dietician vs. } \\
\text { minimal contact control }\end{array}$ & 1 & & Mean Difference (IV, Random, 95\% CI) & $0.0[0.0,0.0]$ \\
\hline $\begin{array}{l}4 \text { BMI percentile change longest } \\
\text { follow-up }\end{array}$ & 1 & & Mean Difference (IV, Fixed, 95\% CI) & Totals not selected \\
\hline 5 BMI change post intervention & 1 & & Mean Difference (IV, Random, 95\% CI) & Totals not selected \\
\hline 6 BMI change longest follow-up & 2 & 614 & Mean Difference (IV, Random, 95\% CI) & $-0.12[-0.39,0.15]$ \\
\hline
\end{tabular}

\section{Comparison 4. Parent-only intervention versus parent-only intervention}

\begin{tabular}{lcccc} 
Outcome or subgroup title & $\begin{array}{c}\text { No. of } \\
\text { studies }\end{array}$ & $\begin{array}{c}\text { No. of } \\
\text { participants }\end{array}$ & Statistical method & Effect size \\
\hline $\begin{array}{l}1 \text { BMI z score change post } \\
\text { intervention }\end{array}$ & 5 & 507 & Mean Difference (IV, Fixed, 95\% CI) & $-0.22[-0.28,-0.17]$ \\
$\begin{array}{l}1.1 \text { Parent-only interactive } \\
\text { voice response vs. parent-only } \\
1.2 \text { Parent-only intensive vs. }\end{array}$ & 1 & 132 & Mean Difference (IV, Fixed, 95\% CI) & $-0.04[-0.16,0.08]$ \\
parent-only & 57 & Mean Difference (IV, Fixed, 95\% CI) & -0.09 [-0.38, 0.20] \\
\hline
\end{tabular}


1.3 Parent health lifestyle vs. healthy lifestyle

1.4 Parent-only vs. decrease

1.5 Parent-only vs. increase

1.6 Parent-only vs. substitute

1.7 Parent-only vs. traditional

2 BMI z score change longest

follow-up

2.1 Parent-only interactive

voice response vs. parent-only

2.2 Parent-only intensive vs. parent-only

2.3 Parent health lifestyle vs. healthy lifestyle

2.4 Parent-only vs. decrease

2.5 Parent-only vs. increase

2.6 Parent-only vs. substitute

2.7 Parent-only vs. traditional

3 BMI change post intervention

4 BMI change longest follow-up

5 BMI percentile change post intervention [\%]
136 Mean Difference (IV, Fixed, 95\% CI)

Mean Difference (IV, Fixed, 95\% CI)

Mean Difference (IV, Fixed, 95\% CI)

Mean Difference (IV, Fixed, 95\% CI)

Mean Difference (IV, Fixed, 95\% CI)

Mean Difference (IV, Fixed, 95\% CI)

467

119 Mean Difference (IV, Fixed, 95\% CI)

Mean Difference (IV, Fixed, 95\% CI)

Mean Difference (IV, Fixed, 95\% CI)

Mean Difference (IV, Fixed, 95\% CI)

Mean Difference (IV, Fixed, 95\% CI)

Mean Difference (IV, Fixed, 95\% CI)

Mean Difference (IV, Fixed, 95\% CI)

Mean Difference (IV, Fixed, 95\% CI)

Mean Difference (IV, Fixed, 95\% CI)

Mean Difference (IV, Random, 95\% CI)
$-0.07[-0.29,0.15]$

$-0.04[-0.17,0.09]$

$-0.01[-0.14,0.12]$

$-0.70[-0.86,-0.54]$

$-0.69[-0.83,-0.55]$

$-0.03[-0.10,0.03]$

$-0.06[-0.18,0.06]$

$-0.09[-0.32,0.14]$

$0.03[-0.24,0.30]$

$-0.04[-0.19,0.11]$

$-0.02[-0.17,0.13]$

$-0.03[-0.24,0.18]$

$0.01[-0.17,0.19]$

Totals not selected

Totals not selected

Totals not selected 
Analysis I.I. Comparison I Parent-only interventions versus parent-child interventions, Outcome I BMI z score change post intervention.

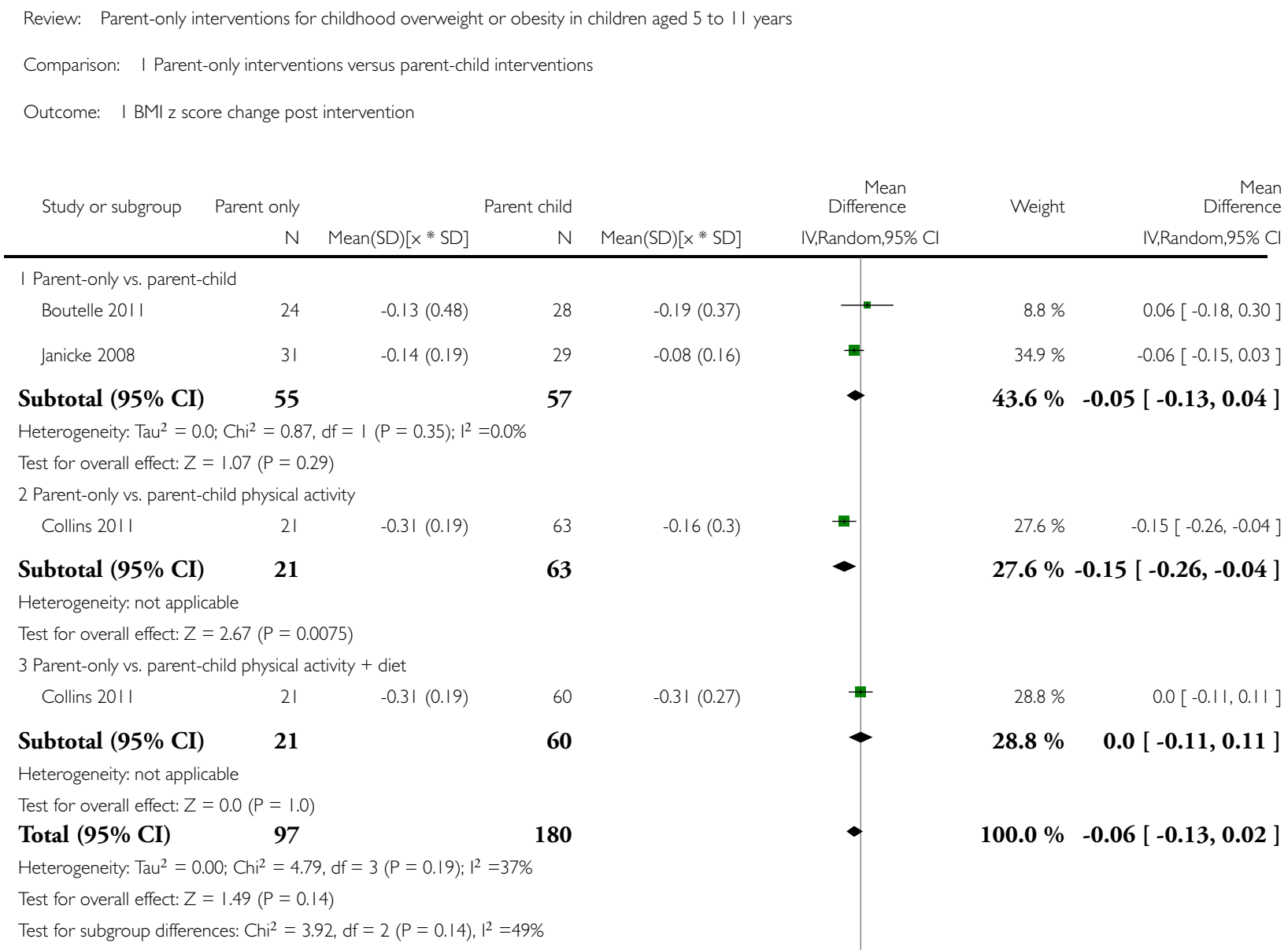

Heterogeneity: not applicable

Test for overall effect: $Z=2.67(P=0.0075)$

3 Parent-only vs. parent-child physical activity + diet

$\begin{array}{lllll}\text { Collins 2011 } & 21 & -0.31(0.19) & 60 & -0.31(0.27)\end{array}$

Subtotal $(95 \% \mathrm{CI}) \quad 21$

Heterogeneity: not applicable

Test for overall effect: $Z=0.0(P=1.0)$

\section{Total (95\% CI) $\quad 97$}

Heterogeneity: $\mathrm{Tau}^{2}=0.00 ; \mathrm{Chi}^{2}=4.79, \mathrm{df}=3(\mathrm{P}=0.19) ; \mathbf{1}^{2}=37 \%$

Test for overall effect: $Z=1.49(P=0.14)$

Test for subgroup differences: $\mathrm{Chi}^{2}=3.92, \mathrm{df}=2(\mathrm{P}=0.14), \mathrm{I}^{2}=49 \%$ 
Analysis I.2. Comparison I Parent-only interventions versus parent-child interventions, Outcome 2 BMI z score change longest follow-up.

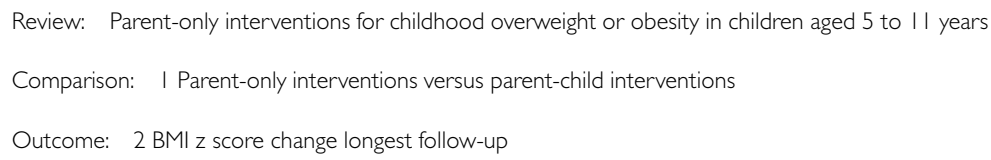

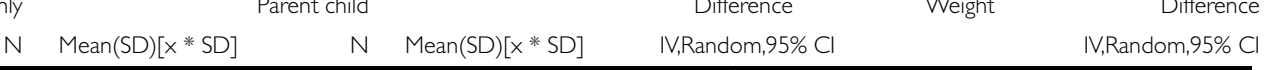

Subtotal (95\% CI)

$$
21
$$

Heterogeneity: not applicable

Test for overall effect: $Z=1.55(P=0.12)$

3 Parent-only vs. parent-child physical activity + diet
Collins 201।
21
$-0.35(0.4)$
60
$-0.24(0.43)$

Subtotal (95\% CI)

$$
21
$$

60

Heterogeneity: not applicable

Test for overall effect: $Z=1.06(P=0.29)$

Total (95\% CI)

Heterogeneity: $\mathrm{Tau}^{2}=0.01 ; \mathrm{Chi}^{2}=4.86, \mathrm{df}=3(\mathrm{P}=0.18) ; \mathrm{I}^{2}=38 \%$

Test for overall effect: $Z=0.59(P=0.56)$

Test for subgroup differences: $\mathrm{Chi}^{2}=4.5 \mathrm{I}, \mathrm{df}=2(\mathrm{P}=0 . \mathrm{II}), \mathrm{I}^{2}=56 \%$

$\begin{array}{ll}14.6 \% & -0.02[-0.29,0.25] \\ 40.2 \% & 0.07[-0.05,0.19]\end{array}$

$54.8 \% \quad 0.06[-0.05,0.16]$

$22.6 \%-0.16[-0.36,0.04]$

$22.6 \%-0.16[-0.36,0.04]$

$22.6 \%-0.11[-0.31,0.09]$

$22.6 \%-0.11[-0.31,0.09]$

$100.0 \%-0.04[-0.15,0.08]$

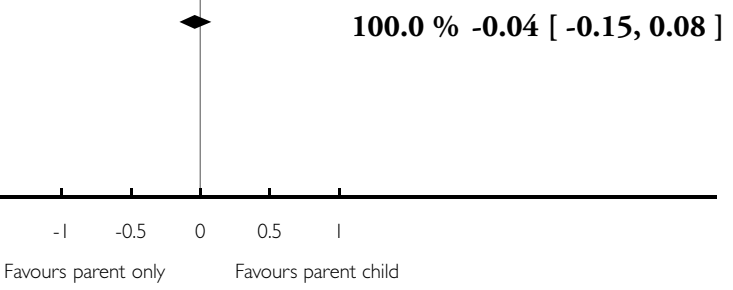


Analysis 2.I. Comparison 2 Parent-only interventions versus waiting list interventions, Outcome I BMI z score change post intervention.

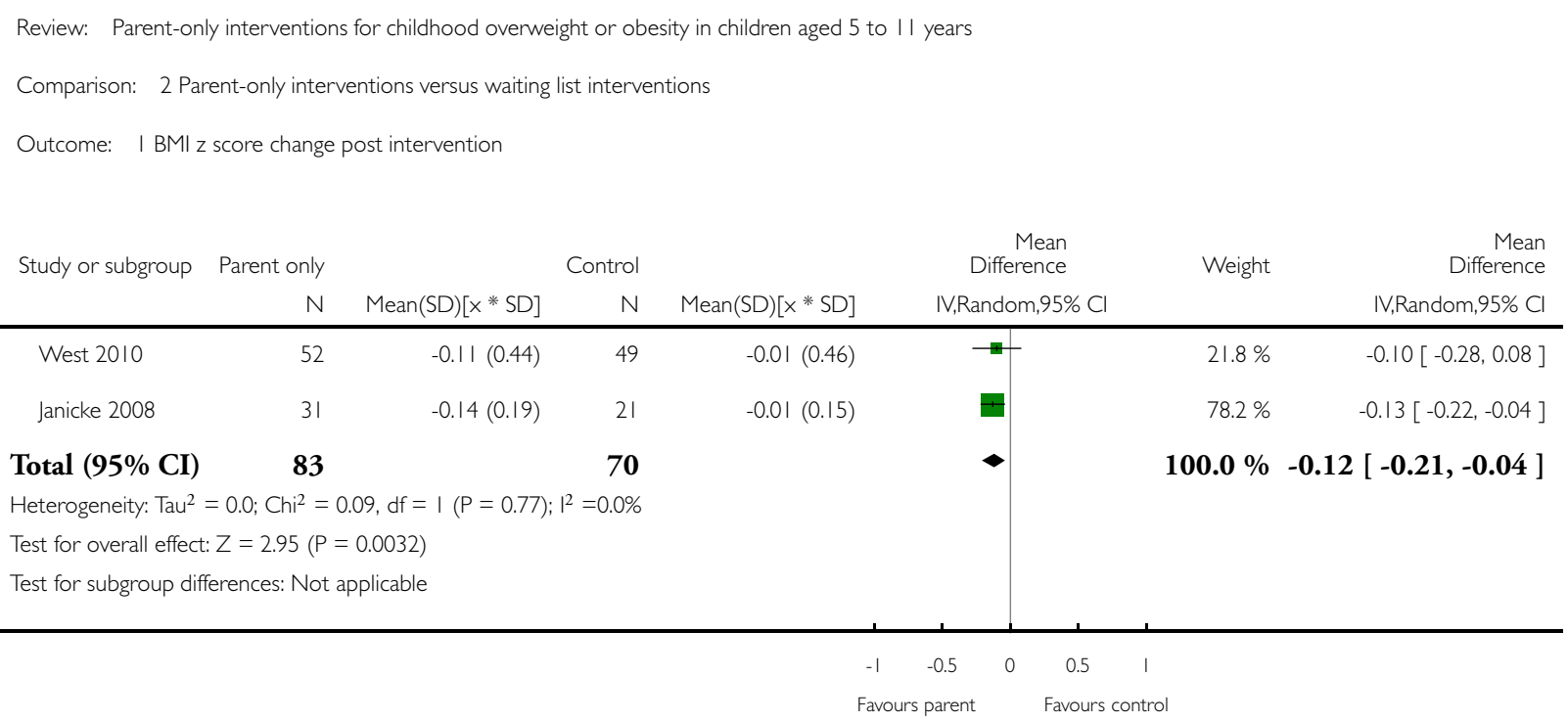


Analysis 2.2. Comparison 2 Parent-only interventions versus waiting list interventions, Outcome 2 BMI z score change longest follow-up.

Review: Parent-only interventions for childhood overweight or obesity in children aged 5 to I I years

Comparison: 2 Parent-only interventions versus waiting list interventions

Outcome: 2 BMI z score change longest follow-up

Study or subgroup Parent only Control Mean Mean

N Mean $(\mathrm{SD})[\mathrm{x} * \mathrm{SD}] \quad \mathrm{N} \quad \operatorname{Mean}(\mathrm{SD})[\mathrm{x} * \mathrm{SD}] \quad$ IV,Fixed,95\% Cl IV,Fixed,95\% Cl

\begin{tabular}{|c|c|c|c|c|c|c|}
\hline \multicolumn{7}{|c|}{ I Parent-only vs. waiting list } \\
\hline Janicke 2008 & 24 & $-0.09(0.2)$ & 21 & $0.02(0.17)$ & $73.7 \%$ & $-0.11[-0.22,0.00]$ \\
\hline Golley 2007 & 31 & $-0.24(0.43)$ & 16 & $-0.13(0.4)$ & $14.0 \%$ & $-0.11[-0.36,0.14]$ \\
\hline
\end{tabular}

Subtotal (95\% CI) $\quad \mathbf{5 5} \quad 37$

Heterogeneity: $\mathrm{Chi}^{2}=0.00, \mathrm{df}=\mathrm{I}(\mathrm{P}=1.00) ; \mathrm{I}^{2}=0.0 \%$

Test for overall effect: $Z=2.18(P=0.030)$

2 Parent-only intensive education vs. waiting list

$\begin{array}{lllll}\text { Golley } 2007 & 29 & -0.15(0.47) & 15 & -0.13(0.4)\end{array}$

Subtotal (95\% CI)

29

$12.3 \%-0.02[-0.29,0.25]$

Heterogeneity: not applicable

Test for overall effect: $Z=0.15(P=0.88)$

Total (95\% CI)

84

$87.7 \%-0.11[-0.21,-0.01]$

Heterogeneity: $\mathrm{Chi}^{2}=0.39, \mathrm{df}=2(\mathrm{P}=0.82) ; \mathrm{I}^{2}=0.0 \%$

Test for overall effect: $Z=2.09(P=0.037)$

Test for subgroup differences: $\mathrm{Chi}^{2}=0.39, \mathrm{df}=\mathrm{I}(\mathrm{P}=0.53), \mathrm{I}^{2}=0.0 \%$

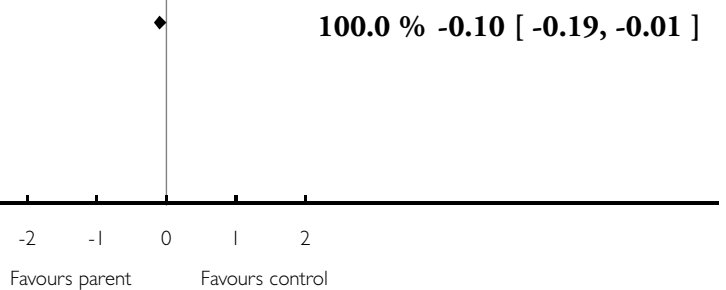


Analysis 2.3. Comparison 2 Parent-only interventions versus waiting list interventions, Outcome 3 BMI percentile change post intervention.

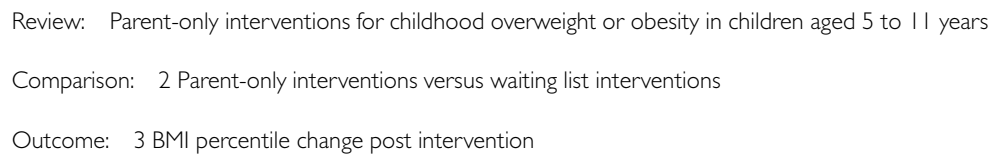

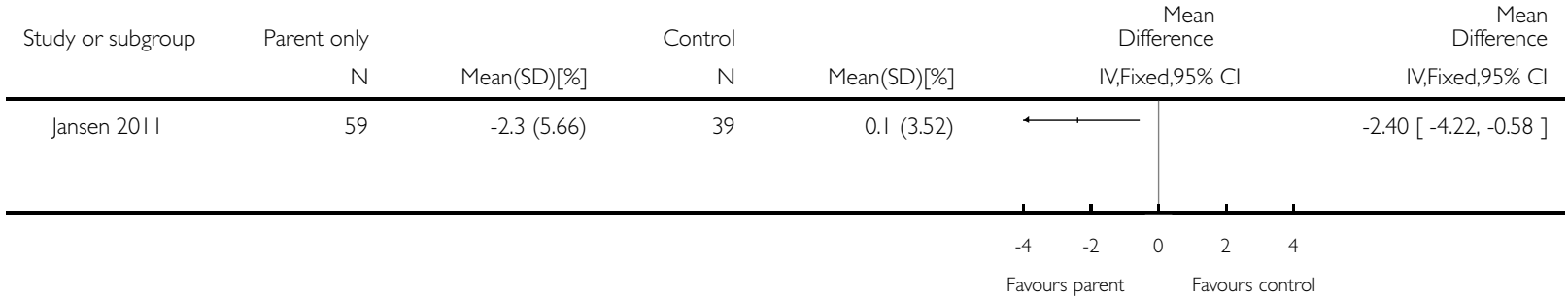

Analysis 2.4. Comparison 2 Parent-only interventions versus waiting list interventions, Outcome 4 BMI percentile change longest follow-up.

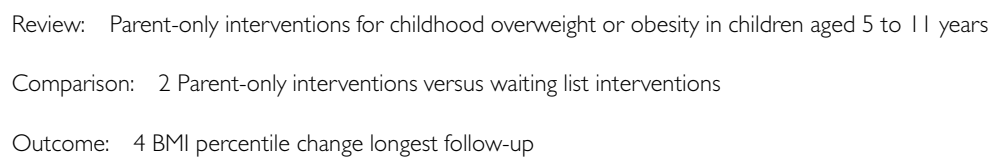

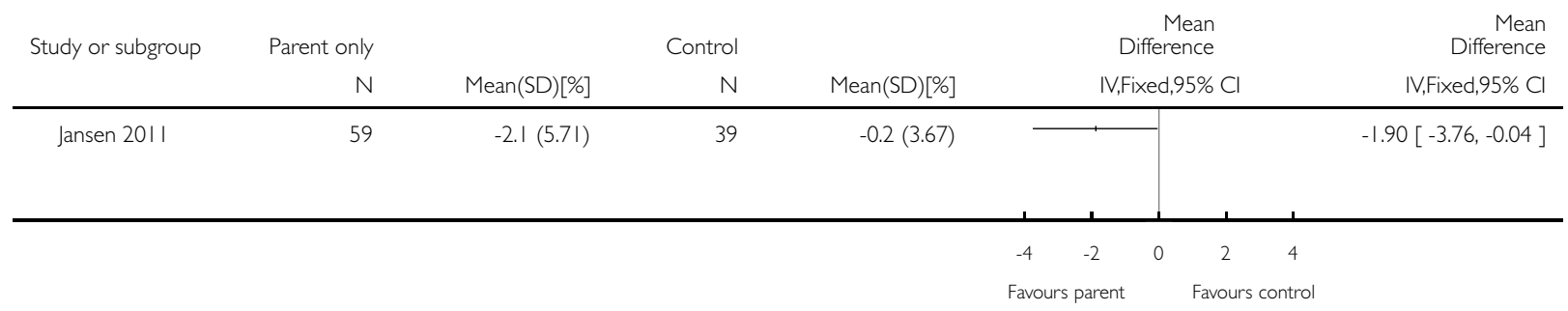


Analysis 2.5. Comparison 2 Parent-only interventions versus waiting list interventions, Outcome 5 BMI change post intervention.

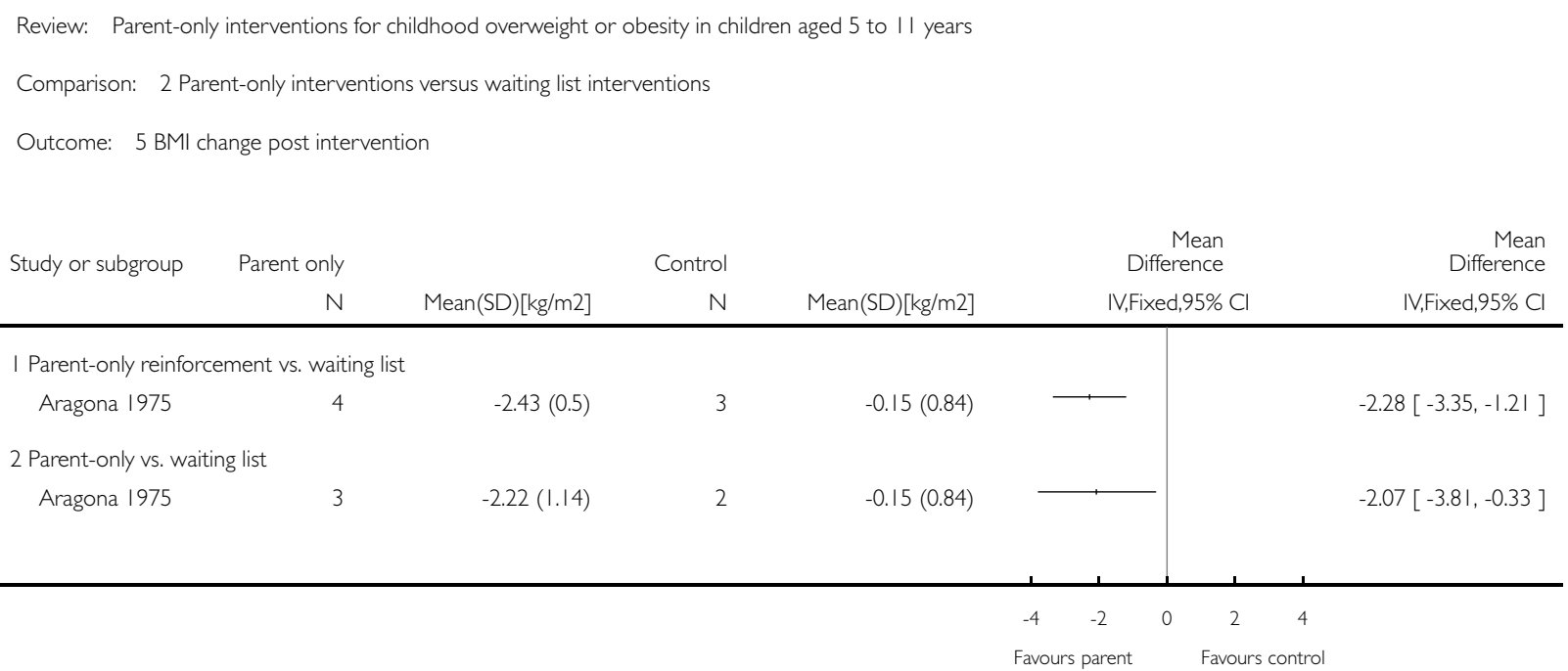

\section{Analysis 2.6. Comparison 2 Parent-only interventions versus waiting list interventions, Outcome 6 BMI} change longest follow-up.

Review: Parent-only interventions for childhood overweight or obesity in children aged 5 to I I years

Comparison: 2 Parent-only interventions versus waiting list interventions

Outcome: 6 BMI change longest follow-up

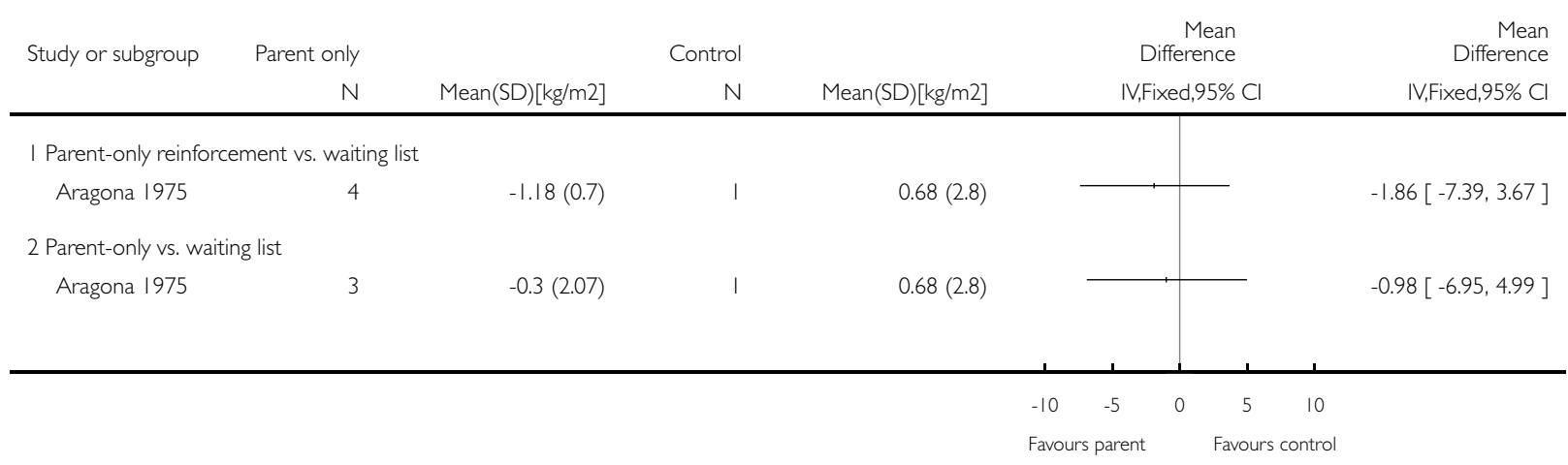


Analysis 3.I. Comparison 3 Parent-only interventions versus minimal contact interventions, Outcome I BMI z score change post intervention.

Review: Parent-only interventions for childhood overweight or obesity in children aged 5 to I I years

Comparison: 3 Parent-only interventions versus minimal contact interventions

Outcome: I BMI z score change post intervention

Study or subgroup Parent only Control Mean Mifference Meight

N Mean(SD)[x*SD] N $\quad$ Mean(SD) $[x * S D] \quad I V, R a n d o m, 95 \%$ Cl IV,Random,95\% Cl

I Parent-only IVR vs. control

$\begin{array}{llll}\text { Estabrooks } 2009 & 68 & -0.07(0.35) & 19\end{array}$

Subtotal (95\% CI)

68

19

Heterogeneity: not applicable

Test for overall effect: $Z=0.36(P=0.72)$

2 Parent-only vs. control

Estabrooks 2009

$\begin{array}{llll}64 & -0.03(0.35) & 19 & -0.05(0.16)\end{array}$

IV,Random,95\% Cl

IV,Random,95\% Cl

Subtotal (95\% CI)

64

19

$50.9 \%$

$-0.02[-0.13,0.09]$

Heterogeneity: not applicable

Test for overall effect: $Z=0.35(P=0.73)$

Total (95\% CI)

132

38

Heterogeneity: $\mathrm{Tau}^{2}=0.0 ; \mathrm{Chi}^{2}=0.25, \mathrm{df}=\mathrm{I}(\mathrm{P}=0.62) ; \mathrm{I}^{2}=0.0 \%$

Test for overall effect: $Z=0.01(P=0.99)$

Test for subgroup differences: $\mathrm{Chi}^{2}=0.25, \mathrm{df}=\mathrm{I}(\mathrm{P}=0.62), \mathrm{I}^{2}=0.0 \%$

(1)

(1)

$49.1 \%$

$0.02[-0.09,0.13]$

$49.1 \% 0.02[-0.09,0.13]$

$50.9 \%-0.02[-0.13,0.09]$

$0.1 \% 0.02[-0.09,0.13]$

$100.0 \% 0.00[-0.08,0.08]$ 
Analysis 3.2. Comparison 3 Parent-only interventions versus minimal contact interventions, Outcome 2 BMI z score change longest follow-up.

Review: Parent-only interventions for childhood overweight or obesity in children aged 5 to I I years

Comparison: 3 Parent-only interventions versus minimal contact interventions

Outcome: $2 \mathrm{BMI}$ z score change longest follow-up

Study or subgroup Parent only Control Mean Difference Weight

N Mean(SD)[x*SD] N $\quad$ Mean(SD) $[x * S D] \quad I V$,Fixed,95\% Cl IV,Fixed,95\% Cl

I Parent-only interactive voice response vs. control

Estabrooks 2009

63

$-0.08(0.35)$

$23-0.06(0.16)$

$51.0 \%$

$-0.02[-0.13,0.09]$

Subtotal (95\% CI)

63

23

Heterogeneity: not applicable

Test for overall effect: $Z=0.36(P=0.72)$

2 Parent-only vs. control

Estabrooks 2009

Subtotal (95\% CI)

56

Heterogeneity: not applicable

Test for overall effect: $Z=0.7 \mid(P=0.48)$

Total (95\% CI)

119

46

Heterogeneity: Chi $^{2}=0.58, d f=I(P=0.45) ;\left.\right|^{2}=0.0 \%$

Test for overall effect: $Z=0.24(P=0.8 \mathrm{I})$

Test for subgroup differences: $\mathrm{Chi}^{2}=0.58, \mathrm{df}=\mathrm{I}(\mathrm{P}=0.45), \mathrm{I}^{2}=0.0 \%$

3

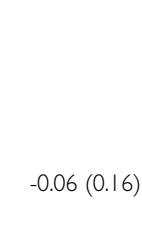

$51.0 \%-0.02[-0.13,0.09]$

$49.0 \%$

$0.04[-0.07,0.15]$

$49.0 \% 0.04[-0.07,0.15]$

$100.0 \% 0.01[-0.07,0.09]$ 
Analysis 3.3. Comparison 3 Parent-only interventions versus minimal contact interventions, Outcome 3 BMI percentile change post intervention.

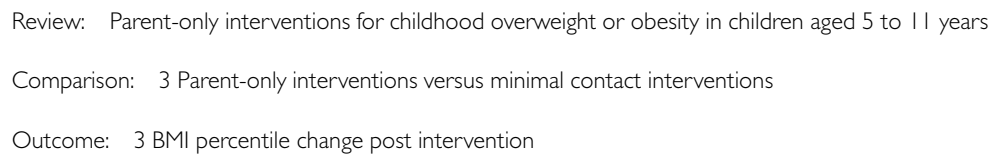

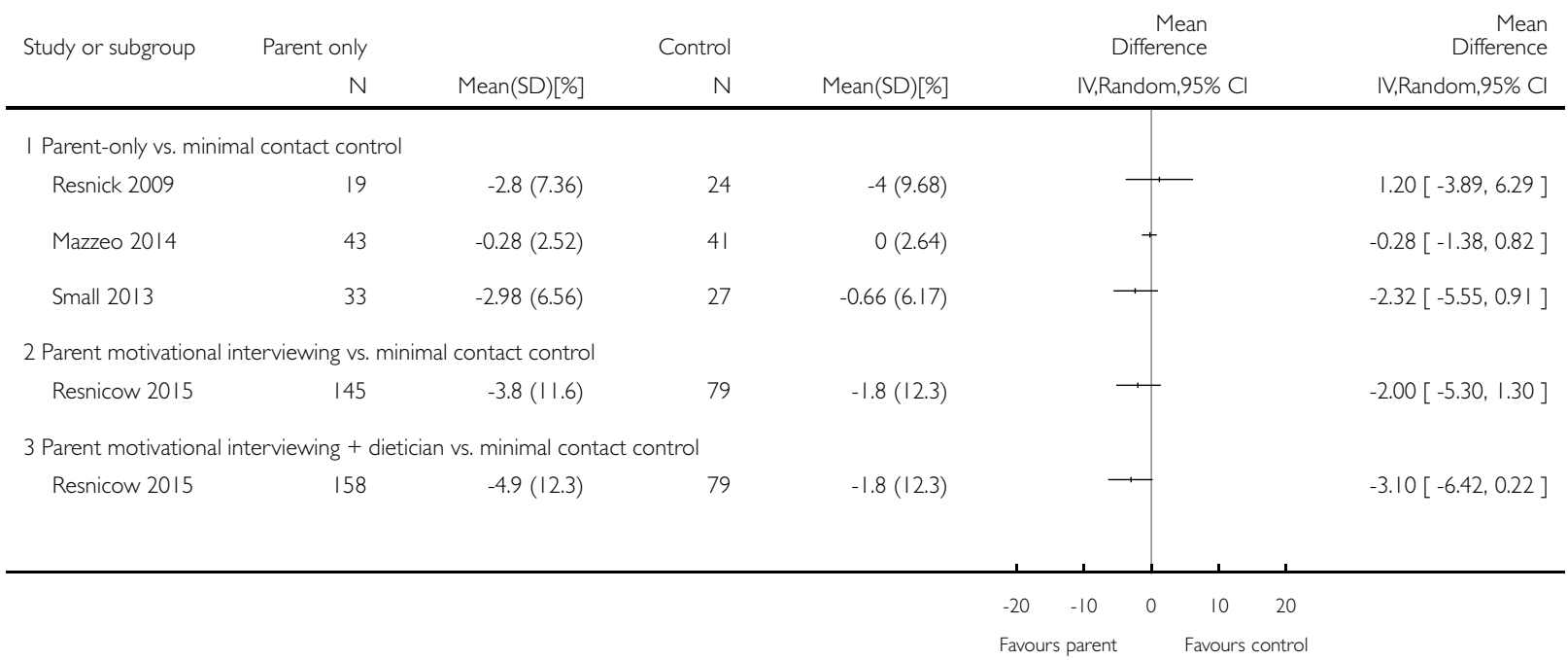

Analysis 3.4. Comparison 3 Parent-only interventions versus minimal contact interventions, Outcome 4 BMI percentile change longest follow-up.

Review: Parent-only interventions for childhood overweight or obesity in children aged 5 to I I years

Comparison: 3 Parent-only interventions versus minimal contact interventions

Outcome: $4 \mathrm{BMI}$ percentile change longest follow-up

\begin{tabular}{|c|c|c|c|c|c|c|c|c|c|c|}
\hline \multirow[t]{2}{*}{ Study or subgroup } & \multirow{2}{*}{$\begin{array}{r}\text { Parent only } \\
\text { N }\end{array}$} & \multicolumn{3}{|c|}{ Control } & \multicolumn{5}{|c|}{$\begin{array}{r}\text { Mean } \\
\text { Difference }\end{array}$} & \multirow{2}{*}{$\begin{array}{r}\text { Mean } \\
\text { Difference } \\
\text { IV,Fixed,95\% Cl }\end{array}$} \\
\hline & & Mean(SD)[\%] & $\mathrm{N}$ & Mean(SD)[\%] & & & xed, & $\% \mathrm{Cl}$ & & \\
\hline \multirow[t]{3}{*}{ Small 2013} & 33 & $-1.59(4.53)$ & 27 & $-0.66(5.4 I)$ & & & + & & & $-0.93[-3.49,1.63]$ \\
\hline & & & & & -50 & -25 & 0 & 25 & 50 & \\
\hline & & & & & Favo & paren & & Favours & ontrol & \\
\hline
\end{tabular}

Parent-only interventions for childhood overweight or obesity in children aged 5 to I I years (Review)

Copyright @ 2015 The Cochrane Collaboration. Published by John Wiley \& Sons, Ltd. 


\section{Analysis 3.5. Comparison 3 Parent-only interventions versus minimal contact interventions, Outcome 5}

BMI change post intervention.

Review: Parent-only interventions for childhood overweight or obesity in children aged 5 to I I years

Comparison: 3 Parent-only interventions versus minimal contact interventions

Outcome: 5 BMl change post intervention

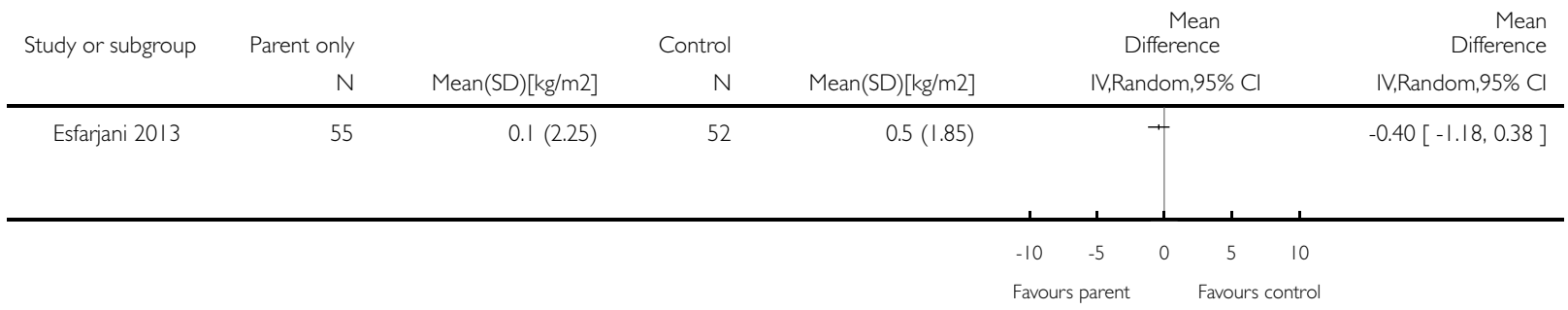


Analysis 3.6. Comparison 3 Parent-only interventions versus minimal contact interventions, Outcome 6 BMI change longest follow-up.

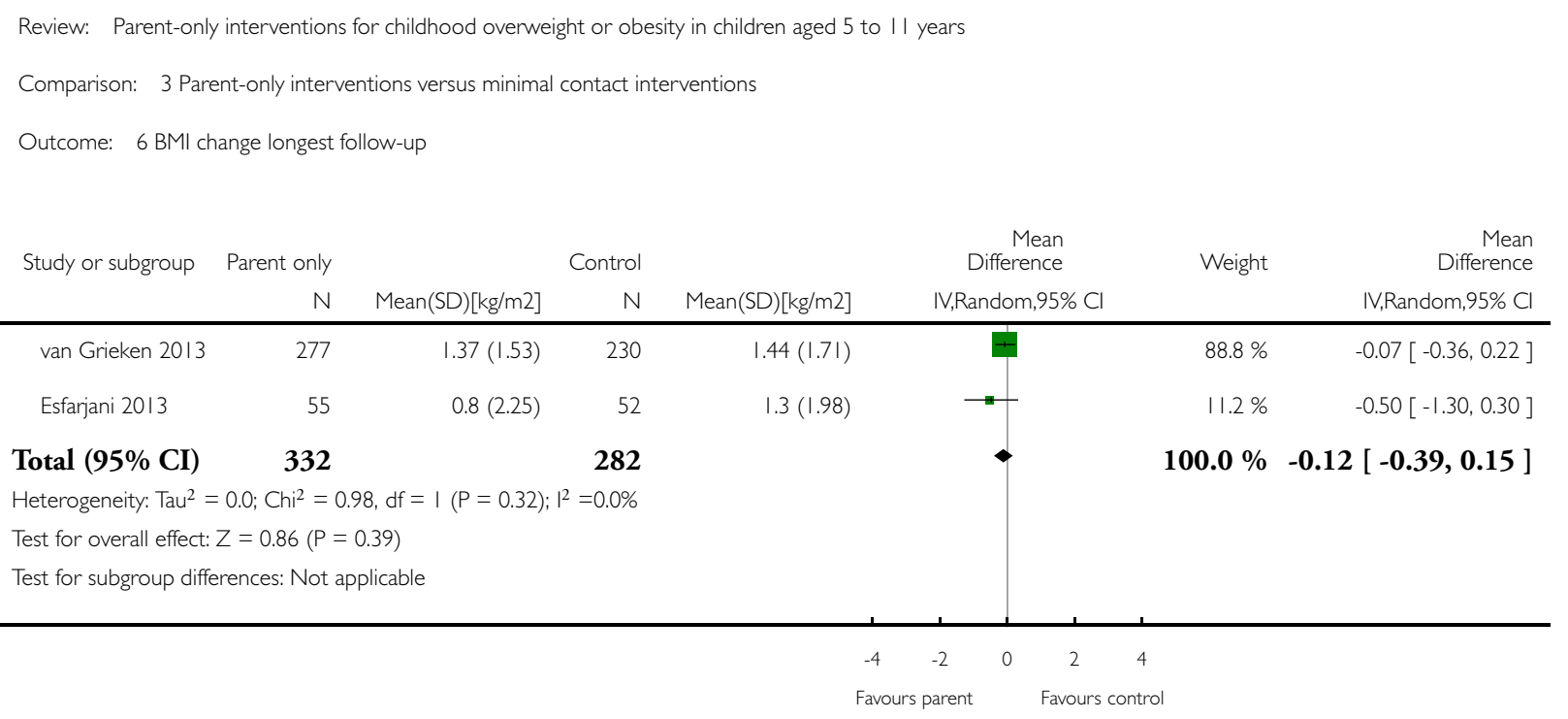




\section{Analysis 4.I. Comparison 4 Parent-only intervention versus parent-only intervention, Outcome I BMI z}

score change post intervention.

Review: Parent-only interventions for childhood overweight or obesity in children aged 5 to I I years

Comparison: 4 Parent-only intervention versus parent-only intervention

Outcome: I BMI z score change post intervention

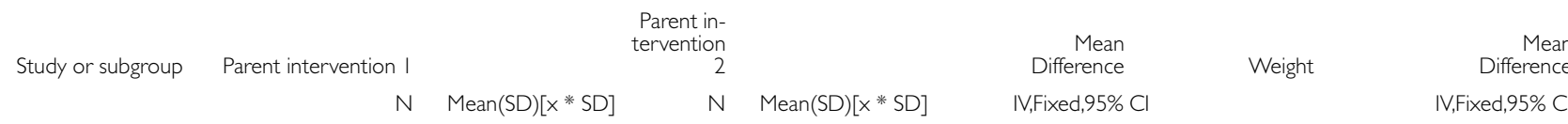

I Parent-only interactive voice response vs. parent-only

Estabrooks 2009

$68-0.07(0.35)$

64

$-0.03(0.35)$

Subtotal $(95 \% \mathrm{CI})$

68

64

Heterogeneity: not applicable

Test for overall effect: $Z=0.66(P=0.5 \mathrm{I})$

2 Parent-only intensive vs. parent-only Golley 2007

28

28

Subtotal (95\% CI)

29

Heterogeneity: not applicable

Test for overall effect: $Z=0.61(P=0.54)$

3 Parent health lifestyle vs. healthy lifestyle

Magarey 2011

Subtotal (95\% CI)

$66-0.29(0.65)$

66

70

70

Heterogeneity: not applicable

Test for overall effect: $Z=0.61(P=0.54)$

4 Parent-only vs. decrease

Raynor 2012a

$-0.11(0.23)$

35

35

Subtotal (95\% CI)

17

Heterogeneity: not applicable

Test for overall effect: $Z=0.62(P=0.53)$

5 Parent-only vs. increase

Raynor 2012a

$-0.11(0.23)$

33

16

33

Subtotal (95\% CI)

16

Heterogeneity: not applicable

Test for overall effect: $Z=0.15(P=0.88)$

6 Parent-only vs. substitute

Raynor 2012b

$-0.8(0.23)$

14

Subtotal (95\% CI)

Heterogeneity: not applicable

Test for overall effect: $Z=8.36(P<0.0000 I)$

7 Parent-only vs. traditional
$22.1 \%$

$-0.04[-0.16,0.08]$

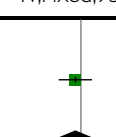

$22.1 \%-0.04[-0.16,0.08]$

$3.7 \%$

$-0.09[-0.38,0.20]$

$3.7 \%-0.09[-0.38,0.20]$

$6.3 \%$

$-0.07[-0.29,0.15]$

$6.3 \%-0.07[-0.29,0.15]$

$19.8 \%$

$-0.04[-0.17,0.09]$

$19.8 \%-0.04[-0.17,0.09]$

$-0.01[-0.14,0.12]$

$19.6 \%-0.01[-0.14,0.12]$

$11.7 \%$

$-0.70[-0.86,-0.54]$

$11.7 \%-0.70[-0.86,-0.54]$

Parent-only interventions for childhood overweight or obesity in children aged 5 to II years (Review)

Copyright $\Subset 2015$ The Cochrane Collaboration. Published by John Wiley \& Sons, Ltd. 


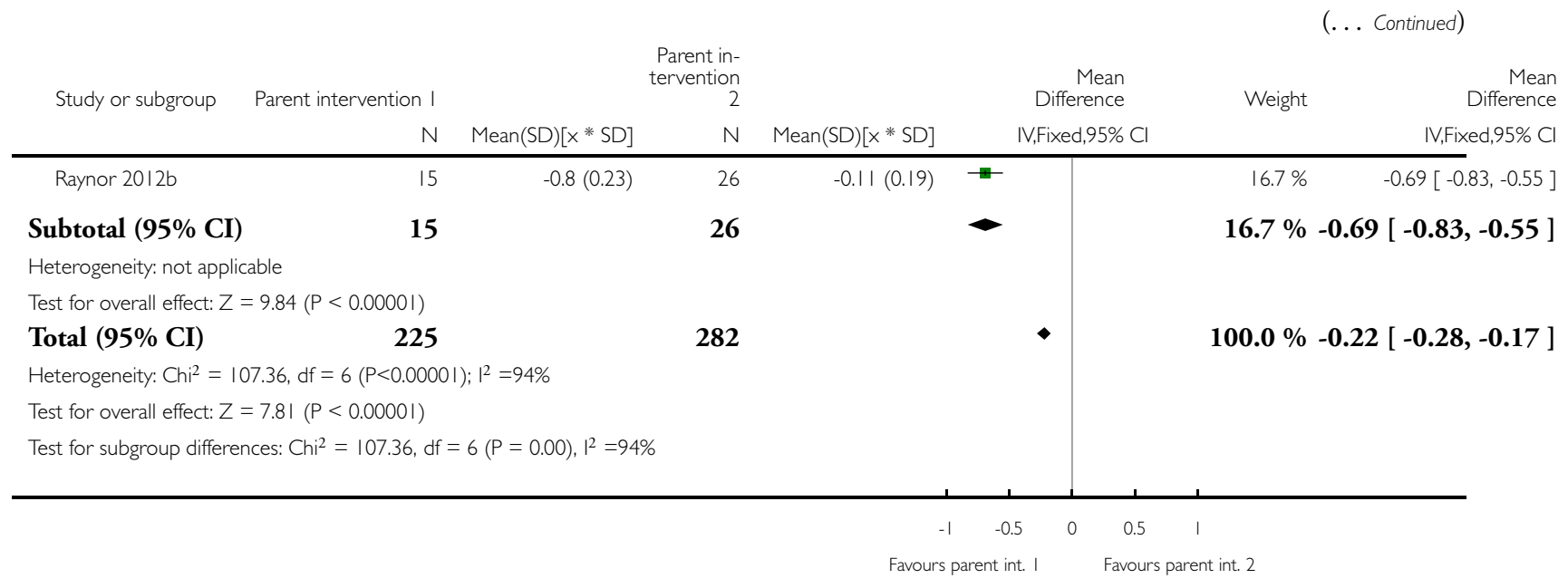

\section{Analysis 4.2. Comparison 4 Parent-only intervention versus parent-only intervention, Outcome 2 BMI z score change longest follow-up.}

Review: Parent-only interventions for childhood overweight or obesity in children aged 5 to $\mid$ I years

Comparison: 4 Parent-only intervention versus parent-only intervention

Outcome: 2 BMI z score change longest follow-up

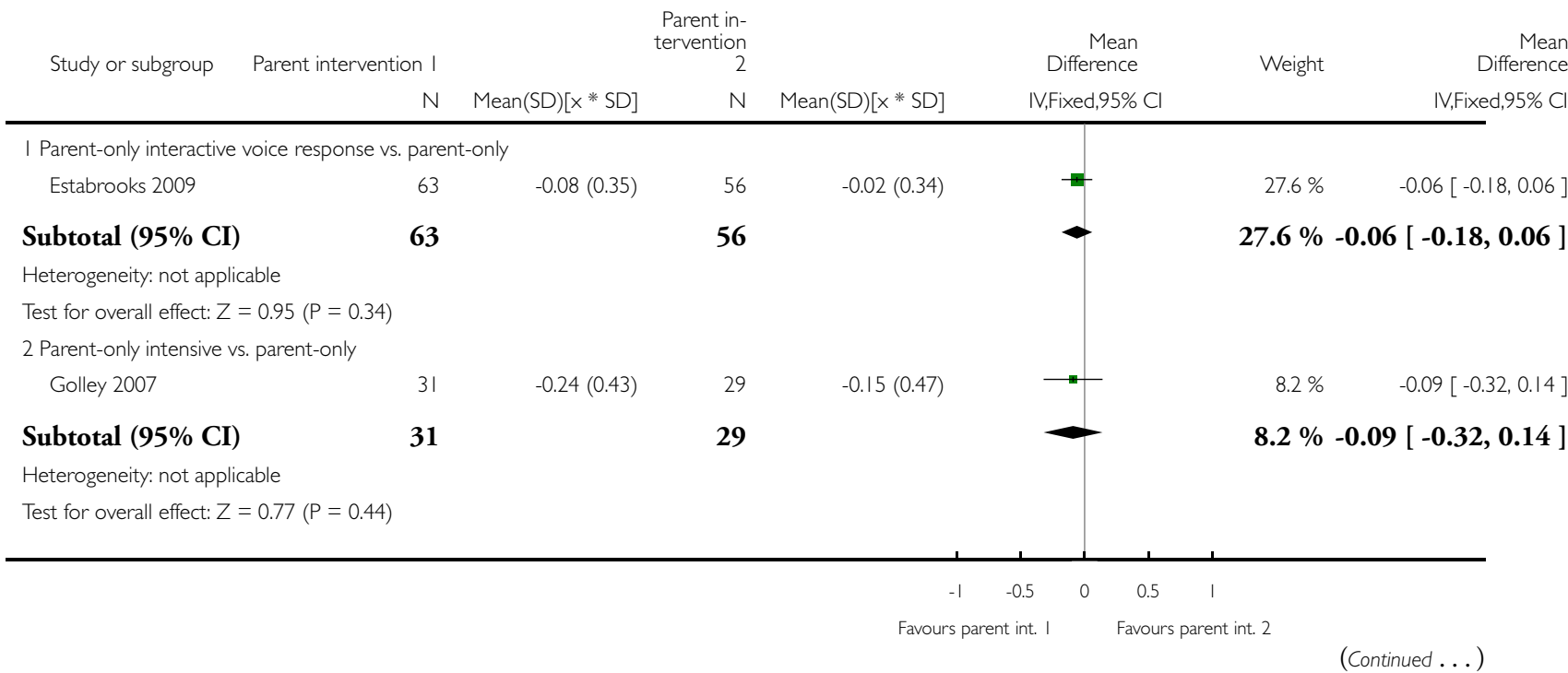

Parent-only interventions for childhood overweight or obesity in children aged 5 to I I years (Review)

Copyright $\Subset 2015$ The Cochrane Collaboration. Published by John Wiley \& Sons, Ltd. 


\begin{tabular}{|c|c|c|c|c|c|c|c|}
\hline \multirow{3}{*}{ Study or subgroup } & \multirow[b]{2}{*}{ Parent intervention I } & \multirow{2}{*}{\multicolumn{2}{|c|}{$\begin{array}{r}\text { Parent in- } \\
\text { tervention } \\
2\end{array}$}} & & \multirow[b]{2}{*}{$\begin{array}{r}\text { Mean } \\
\text { Difference }\end{array}$} & \multicolumn{2}{|r|}{ (... Continued) } \\
\hline & & & & & & Weight & $\begin{array}{r}\text { Mean } \\
\text { Difference }\end{array}$ \\
\hline & N & $\operatorname{Mean}(S D)[x * S D]$ & N & $\operatorname{Mean}(\mathrm{SD})[\mathrm{x} * \mathrm{SD}]$ & IV,Fixed,95\% Cl & & IV,Fixed,95\% Cl \\
\hline \multicolumn{8}{|c|}{3 Parent health lifestyle vs. healthy lifestyle } \\
\hline Magarey 2011 & 52 & $-0.39(0.63)$ & 54 & $-0.42(0.76)$ & ( & $6.0 \%$ & $0.03[-0.24,0.30]$ \\
\hline Subtotal $(95 \% \mathrm{CI})$ & 52 & & 54 & & - & $6.0 \%$ & $0.03[-0.24,0.30]$ \\
\hline \multicolumn{8}{|c|}{ Heterogeneity: not applicable } \\
\hline \multicolumn{8}{|c|}{ Test for overall effect: $Z=0.22(P=0.82)$} \\
\hline \multicolumn{8}{|l|}{4 Parent-only vs. decrease } \\
\hline Raynor 2012a & 17 & $-0.14(0.29)$ & 35 & $-0.1(0.21)$ & $\rightarrow$ & $17.8 \%$ & $-0.04[-0.19,0.11]$ \\
\hline Subtotal $(95 \% \mathrm{CI})$ & 17 & & 35 & & & $17.8 \%$ & $-0.04[-0.19,0.11]$ \\
\hline \multicolumn{8}{|c|}{ Heterogeneity: not applicable } \\
\hline \multicolumn{8}{|c|}{ Test for overall effect: $Z=0.51(P=0.61)$} \\
\hline \multicolumn{8}{|l|}{5 Parent-only vs. increase } \\
\hline Raynor 2012a & 16 & $-0.14(0.29)$ & 33 & $-0.12(0.17)$ & - & $18.1 \%$ & $-0.02[-0.17,0.13]$ \\
\hline Subtotal $(95 \% \mathrm{CI})$ & 16 & & 33 & & & $18.1 \%$ & $-0.02[-0.17,0.13]$ \\
\hline \multicolumn{8}{|c|}{ Heterogeneity: not applicable } \\
\hline \multicolumn{8}{|c|}{ Test for overall effect: $Z=0.26(P=0.80)$} \\
\hline \multicolumn{8}{|c|}{6 Parent-only vs. substitute } \\
\hline Raynor 2012b & 15 & $-0.16(0.29)$ & 26 & $-0.13(0.39)$ & - & $9.7 \%$ & $-0.03[-0.24,0.18]$ \\
\hline Subtotal $(95 \% \mathrm{CI})$ & 15 & & 26 & & & $9.7 \%$ & $-0.03[-0.24,0.18]$ \\
\hline \multicolumn{8}{|c|}{ Heterogeneity: not applicable } \\
\hline \multicolumn{8}{|c|}{ Test for overall effect: $Z=0.28(P=0.78)$} \\
\hline \multicolumn{8}{|l|}{7 Parent-only vs. traditional } \\
\hline Raynor 2012b & 14 & $-0.16(0.29)$ & 26 & $-0.17(0.27)$ & - & $12.6 \%$ & $0.01[-0.17,0.19]$ \\
\hline Subtotal $(95 \% \mathrm{CI})$ & 14 & & 26 & & & $12.6 \%$ & $0.01[-0.17,0.19]$ \\
\hline \multicolumn{8}{|c|}{ Heterogeneity: not applicable } \\
\hline \multicolumn{8}{|c|}{ Test for overall effect: $Z=0.11(P=0.92)$} \\
\hline Total $(95 \% \mathrm{CI})$ & 208 & & 259 & & $\bullet$ & $100.0 \%$ & $-0.03[-0.10,0.03]$ \\
\hline \multicolumn{8}{|c|}{ Heterogeneity: $\mathrm{Chi}^{2}=0.88, \mathrm{df}=6(\mathrm{P}=0.99) ; \mathrm{I}^{2}=0.0 \%$} \\
\hline \multicolumn{8}{|c|}{ Test for overall effect: $Z=1.04(P=0.30)$} \\
\hline Test for subgroup differer & nces: Chi $^{2}=0.88, \mathrm{df}=$ & $(P=0.99), I^{2}=0.0 \%$ & & & & & \\
\hline
\end{tabular}


Analysis 4.3. Comparison 4 Parent-only intervention versus parent-only intervention, Outcome 3 BMI change post intervention.

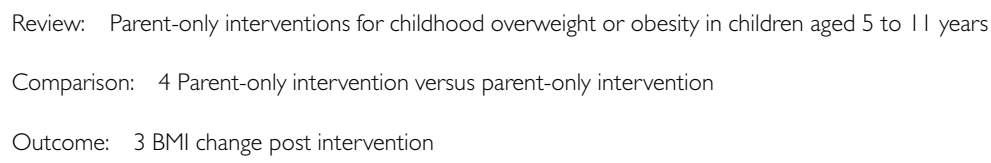

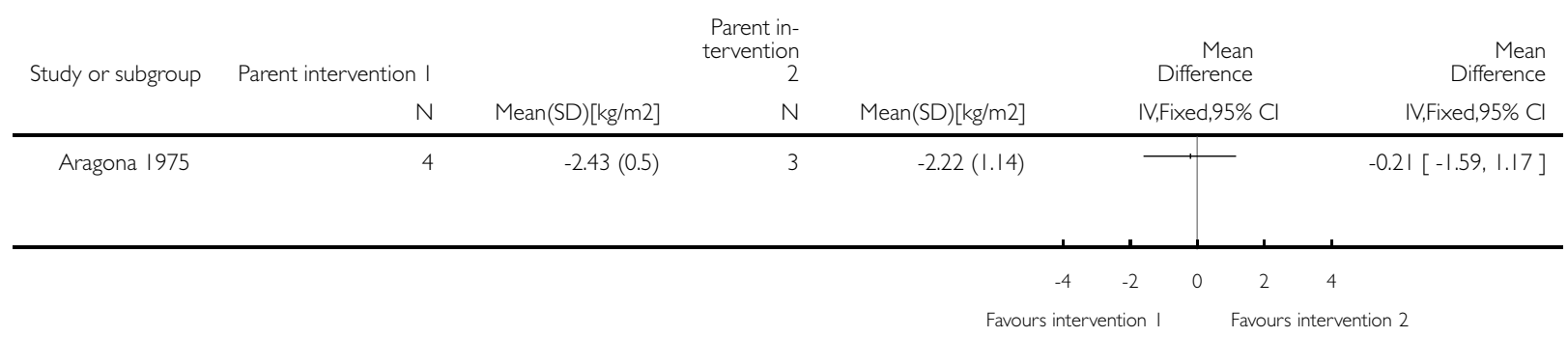

Analysis 4.4. Comparison 4 Parent-only intervention versus parent-only intervention, Outcome 4 BMI change longest follow-up.

Review: Parent-only interventions for childhood overweight or obesity in children aged 5 to II years

Comparison: 4 Parent-only intervention versus parent-only intervention

Outcome: 4 BMl change longest follow-up

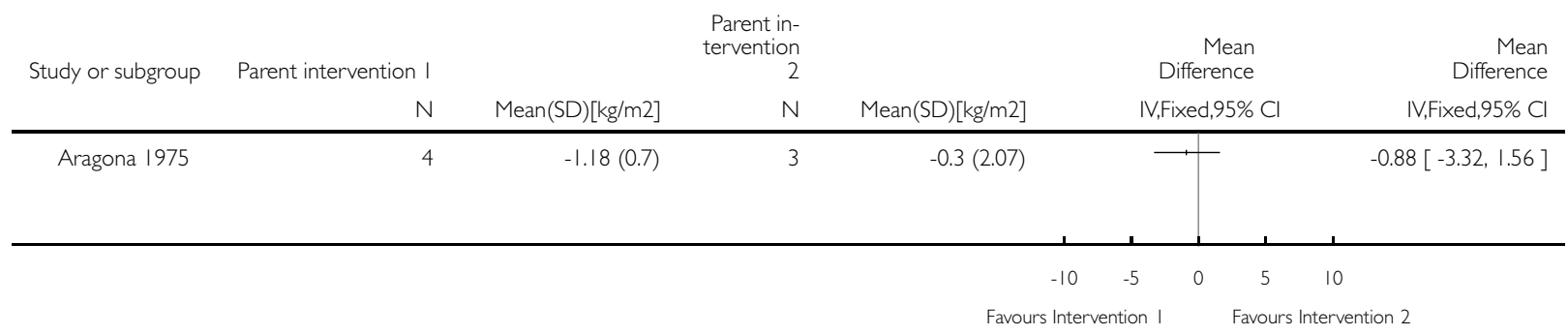


Analysis 4.5. Comparison 4 Parent-only intervention versus parent-only intervention, Outcome 5 BMI percentile change post intervention [\%].

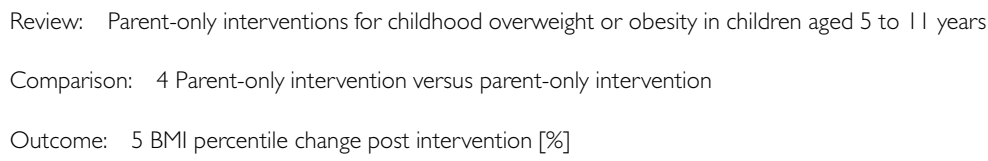

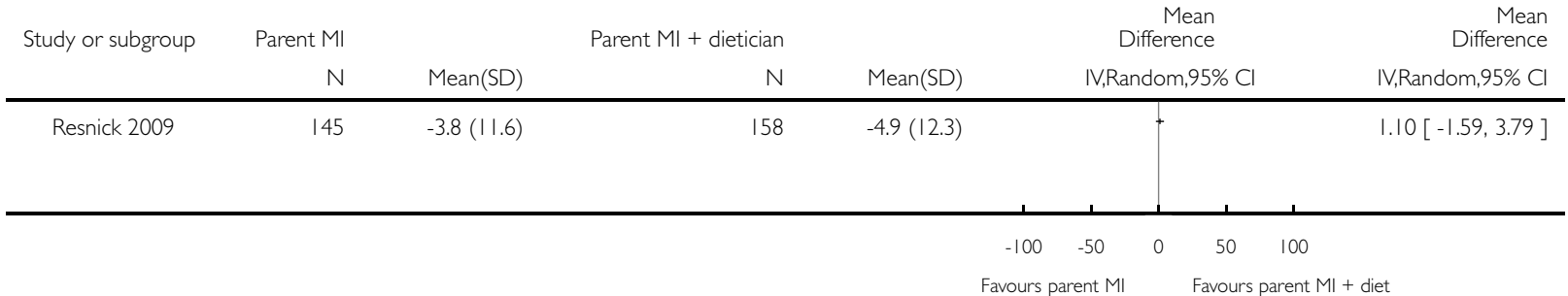

\section{ADDITIONAL TABLES}

Table 1. Overview of study populations

\begin{tabular}{|c|c|c|c|c|c|c|c|c|c|}
\hline & $\begin{array}{l}\text { Interven- } \\
\text { tion(s) } \\
\text { and com- } \\
\text { parator(s) }\end{array}$ & $\begin{array}{l}\text { Sample } \\
\text { size }^{a}\end{array}$ & $\begin{array}{l}\text { Screened/ } \\
\text { eligible } \\
{[\mathrm{N}]}\end{array}$ & $\begin{array}{l}\text { Ran- } \\
\text { domised } \\
{[\mathrm{N}]}\end{array}$ & $\begin{array}{l}\text { ITT } \\
{[\mathbf{N}]}\end{array}$ & $\begin{array}{l}\text { Analysed } \\
{[\mathrm{N}]}\end{array}$ & $\begin{array}{l}\text { Finishing } \\
\text { trial } \\
{[\mathrm{N}]}\end{array}$ & $\begin{array}{l}\text { Ran- } \\
\text { domised } \\
\text { finishing } \\
\text { trial } \\
{[\%]}\end{array}$ & $\begin{array}{l}\text { Follow-up } \\
\text { (ex- } \\
\text { tended fol- } \\
\text { low-up) }{ }^{a}\end{array}$ \\
\hline $\begin{array}{l}(20) \\
\text { Resnicow } \\
2015\end{array}$ & $\begin{array}{l}\text { I1: parent- } \\
\text { only PCP } \\
\text { motiva- } \\
\text { tional in- } \\
\text { terviewing }\end{array}$ & $\begin{array}{l}\text { The study } \\
\text { was pow- } \\
\text { ered to } \\
\text { detect a } \\
\text { 3-point } \\
\text { difference } \\
\text { in BMI } \\
\text { percentile } \\
\text { between } \\
\text { any pair } \\
\text { of study } \\
\text { groups } \\
\text { at 2-year } \\
\text { follow-up, } \\
\text { with an as- } \\
\text { sumed SD } \\
\text { for BMI } \\
\text { percentile } \\
\text { between }\end{array}$ & - & $\begin{array}{l}16 \\
\text { practices } \\
212 \text { partic- } \\
\text { ipants }\end{array}$ & 145 & 145 & 145 & 68 & $\begin{array}{l}2 \text { years }(2 \\
\text { years })\end{array}$ \\
\hline
\end{tabular}


Table 1. Overview of study populations (Continued)

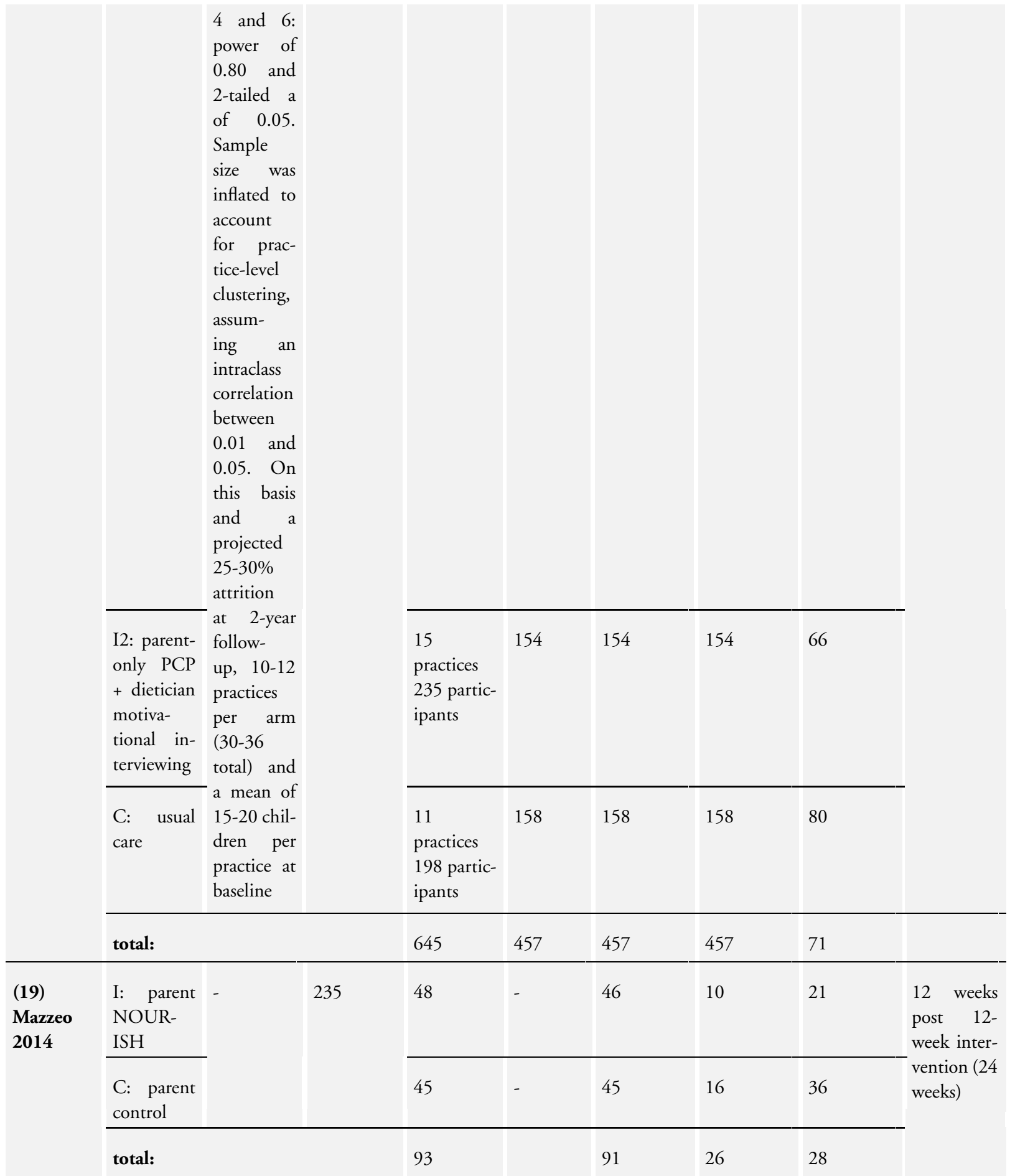


Table 1. Overview of study populations (Continued)

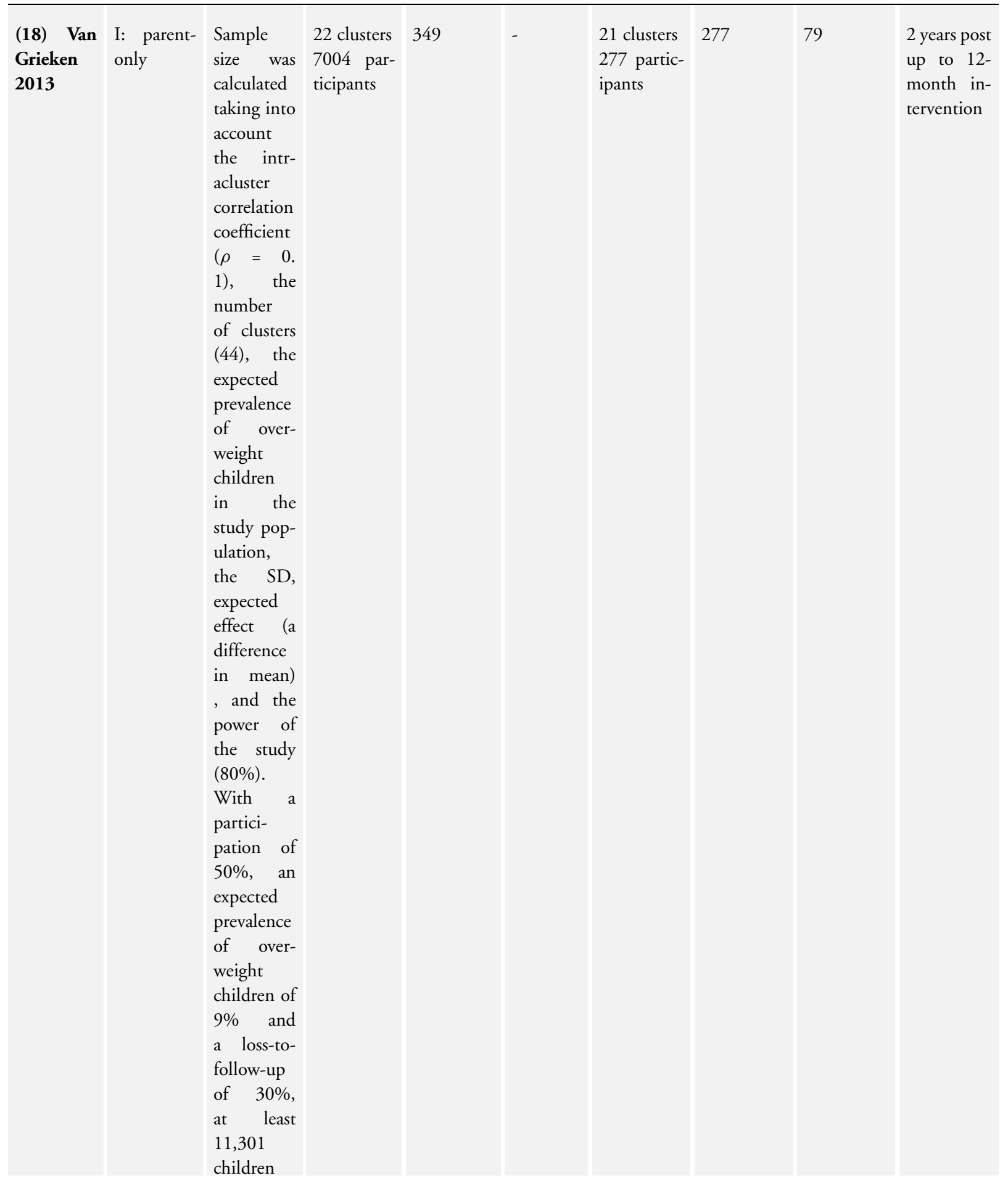


Table 1. Overview of study populations (Continued)

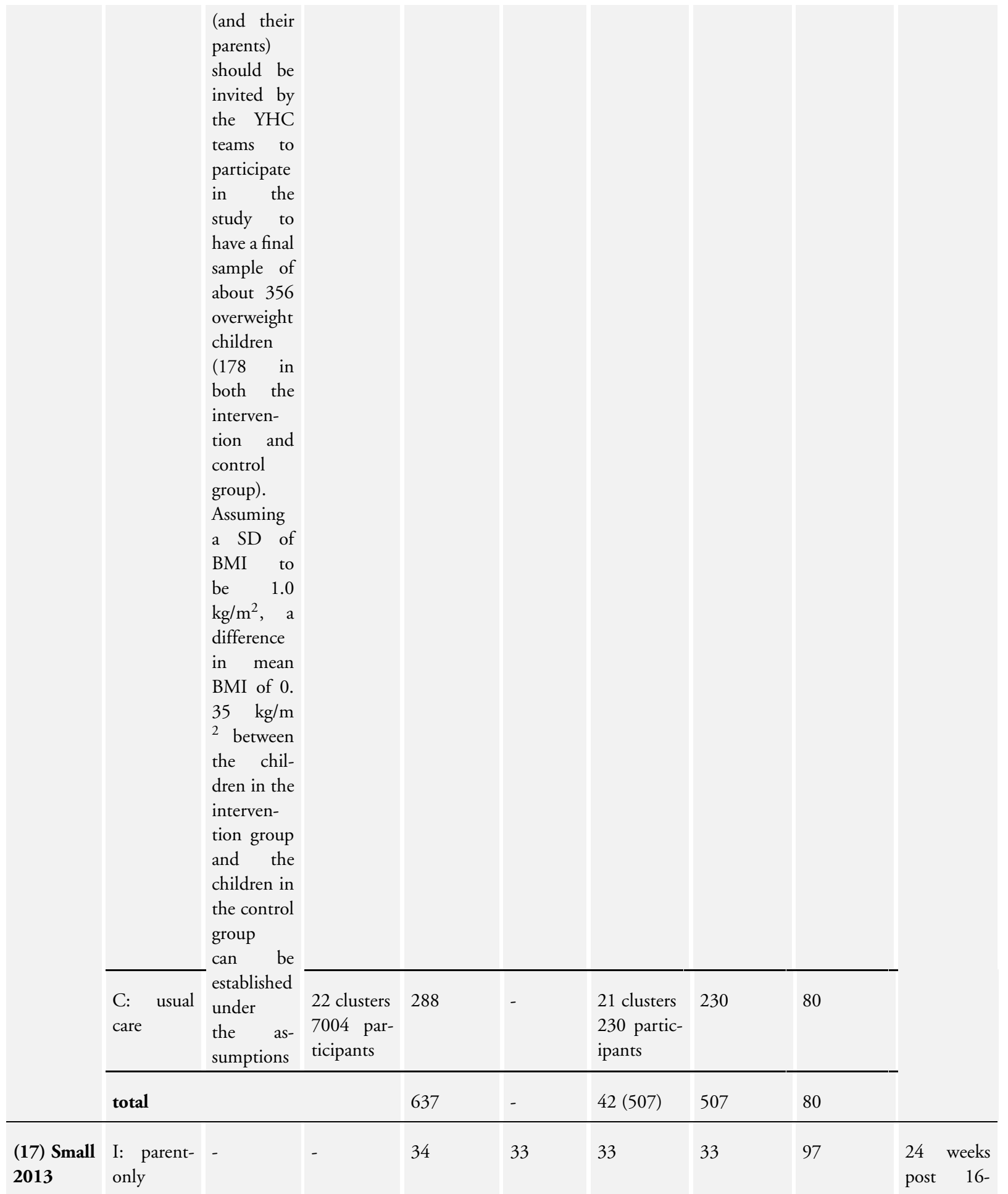

Parent-only interventions for childhood overweight or obesity in children aged 5 to I I years (Review)

Copyright $(\subset) 2015$ The Cochrane Collaboration. Published by John Wiley \& Sons, Ltd. 
Table 1. Overview of study populations (Continued)

\begin{tabular}{|c|c|c|c|c|c|c|c|c|c|}
\hline & \multirow{2}{*}{$\begin{array}{l} \\
\text { C: parent } \\
\text { control }\end{array}$} & & & \multirow[b]{2}{*}{33} & \multirow[b]{2}{*}{27} & & \multirow[b]{2}{*}{82} & \multirow[t]{3}{*}{$\begin{array}{l}\text { week inter- } \\
\text { vention ( } 41 \\
\text { weeks) }\end{array}$} \\
\hline & & & & & & 27 & 27 & & \\
\hline & total: & & & 67 & 60 & 60 & 60 & 90 & \\
\hline \multirow{3}{*}{$\begin{array}{l}(16) \\
\text { Esfarjani } \\
2013\end{array}$} & $\begin{array}{l}\text { I: parent- } \\
\text { only }\end{array}$ & - & $550 / 156$ & 70 & - & 55 & 58 & 83 & \multirow{3}{*}{$\begin{array}{l}\text { Interven- } \\
\text { tion } 6 \\
\text { months } \\
\text { (not } \\
\text { reported) }\end{array}$} \\
\hline & $\begin{array}{l}\text { C: parent } \\
\text { control }\end{array}$ & & & 86 & - & 52 & 59 & 69 & \\
\hline & \multicolumn{3}{|l|}{ total: } & 156 & - & 107 & 117 & 75 & \\
\hline \multirow{3}{*}{$\begin{array}{l}(15) \\
\text { Moens } \\
2012\end{array}$} & $\begin{array}{l}\text { I: parent- } \\
\text { only }\end{array}$ & - & $80 / 75$ & 31 & - & - & 31 & 100 & \multirow{3}{*}{$\begin{array}{l}\text { Imme- } \\
\text { diately fol- } \\
\text { lowing 6- } \\
\text { month in- } \\
\text { tervention }\end{array}$} \\
\hline & $\begin{array}{l}\text { C: waiting } \\
\text { list control }\end{array}$ & & & 19 & - & - & 15 & 79 & \\
\hline & \multicolumn{3}{|l|}{ total: } & 50 & - & - & 46 & 92 & \\
\hline $\begin{array}{l}(14) \\
\text { Raynor } \\
2012 a\end{array}$ & $\begin{array}{l}\text { I1: parent- } \\
\text { only }\end{array}$ & $\begin{array}{l}\text { Sample } \\
\text { size cal- } \\
\text { culations } \\
\text { presumed } \\
\text { 2-sided } \\
\text { hypothesis } \\
\text { testing at } \\
\text { 6-month } \\
\text { assess- } \\
\text { ment, with } \\
\text { type } 1 \\
\text { error rate } \\
=\quad 0.05 . \\
\text { To reject } \\
\text { with } 80 \% \\
\text { power } \\
\text { the null } \\
\text { hypothesis } \\
\text { of no pre- } \\
\text { to post- } \\
\text { treatment } \\
\text { difference } \\
\text { between } \\
\text { inter- }\end{array}$ & 549 & 33 & 33 & 33 & 29 & 88 & $\begin{array}{l}24 \text { weeks } \\
\text { post } 24- \\
\text { week inter- } \\
\text { vention (re- } \\
\text { ported as } \\
\text { '12 } \\
\text { months') }\end{array}$ \\
\hline
\end{tabular}


Table 1. Overview of study populations (Continued)

\begin{tabular}{|c|c|c|c|c|c|c|c|c|c|}
\hline & & $\begin{array}{l}\text { vention } \\
\text { conditions } \\
\text { vs. the } \\
\text { alternative } \\
\text { that the } \\
\text { pre- to }\end{array}$ & & & & & & & \\
\hline & $\begin{array}{l}\text { I2: parent } \\
\text { - diet de- } \\
\text { crease }\end{array}$ & $\begin{array}{l}\text { treatment } \\
\text { difference } \\
\text { was } \quad 0.6\end{array}$ & & 33 & 33 & 33 & 29 & 88 & \\
\hline & $\begin{array}{l}\text { I3: parent } \\
\text { - diet in- } \\
\text { crease }\end{array}$ & $\begin{array}{l}\text { (effect size) } \\
, 24 \text { partic- } \\
\text { ipants per }\end{array}$ & & 35 & 35 & 35 & 32 & 91 & \\
\hline & total: & & & 101 & 101 & 101 & 90 & 89 & \\
\hline $\begin{array}{l}(13) \\
\text { Raynor } \\
2012 b\end{array}$ & $\begin{array}{l}\text { I1: parent- } \\
\text { only }\end{array}$ & $\begin{array}{l}\text { Sample } \\
\text { size cal- } \\
\text { culations } \\
\text { presumed } \\
\text { 2-sided } \\
\text { hypothesis } \\
\text { testing at } \\
\text { 6-month } \\
\text { assess- } \\
\text { ment, with } \\
\text { type } 1 \\
\text { error rate } \\
= \\
\text { To reject } \\
\text { with } 80 \% \\
\text { power } \\
\text { the null } \\
\text { hypothesis } \\
\text { of no pre- } \\
\text { to post- } \\
\text { treatment } \\
\text { difference } \\
\text { between } \\
\text { inter- } \\
\text { vention } \\
\text { conditions } \\
\text { vs. the } \\
\text { alternative } \\
\text { that the } \\
\text { pre- to } \\
\text { post- }\end{array}$ & 549 & 29 & 29 & 29 & 26 & 90 & $\begin{array}{l}24 \text { weeks } \\
\text { post 24- } \\
\text { week inter- } \\
\text { vention (re- } \\
\text { ported as } \\
\text { '12 } \\
\text { months') }\end{array}$ \\
\hline
\end{tabular}


Table 1. Overview of study populations (Continued)

\begin{tabular}{|c|c|c|c|c|c|c|c|c|c|}
\hline & \multirow[b]{2}{*}{$\begin{array}{l}\text { I2: parent } \\
\text { - diet and } \\
\text { activity } \\
\text { traditional }\end{array}$} & \multirow{3}{*}{$\begin{array}{l}\text { treatment } \\
\text { difference } \\
\text { is } 0.6 \text { or } \\
\text { greater } \\
\text { (effect size) } \\
, 24 \text { partic- } \\
\text { ipants per } \\
\text { group were } \\
\text { needed }\end{array}$} & & \multirow[b]{2}{*}{26} & \multirow[b]{2}{*}{26} & \multirow[b]{2}{*}{26} & \multirow[b]{2}{*}{24} & \multirow[b]{2}{*}{92} & \\
\hline & & & & & & & & & \\
\hline & $\begin{array}{l}\text { I3: parent } \\
\text { - diet and } \\
\text { activity } \\
\text { substitute }\end{array}$ & & & 26 & 26 & 26 & 24 & 92 & \\
\hline & total: & & & 81 & 81 & 81 & 74 & 91 & \\
\hline $\begin{array}{l}\text { (12) Mar- } \\
\text { garey } \\
2011\end{array}$ & $\begin{array}{l}\text { I: par- } \\
\text { ent healthy } \\
\text { lifestyle }\end{array}$ & $\begin{array}{l}\text { Sample } \\
\text { size calcu- } \\
\text { lation was } \\
\text { based on a } \\
\text { reduction } \\
\text { in BMI z } \\
\text { score of } 0 . \\
26 \text { (SD } 0 . \\
49 \text { ) over } \\
12 \text { months } \\
\text { (power } \\
80 \% \text { al- } \\
\text { pha } 0.05 \text {, } \\
\text { and drop- } \\
\text { out rate of } \\
30 \% \text { ). } \\
\text { This repre- } \\
\text { sents } \\
\text { a } 50 \% \text { re- } \\
\text { duction in } \\
\text { weight ve- } \\
\text { locity over } \\
12 \text { months } \\
\text { and no } \\
\text { change in } \\
\text { height ve- } \\
\text { locity. We } \\
\text { sought } 42 \\
\text { children } \\
\text { per group } \\
\text { per site } \\
\text { (168 chil- } \\
\text { dren) }\end{array}$ & 398 & 85 & 85 & 85 & 52 & 61 & $\begin{array}{l}80 \text { weeks } \\
\text { post 24- } \\
\text { week inter- } \\
\text { vention } \\
(104 \\
\text { weeks })\end{array}$ \\
\hline
\end{tabular}


Table 1. Overview of study populations (Continued)

\begin{tabular}{|c|c|c|c|c|c|c|c|c|c|}
\hline & $\begin{array}{l}\text { C: healthy } \\
\text { lifestyle }\end{array}$ & & & 84 & 84 & 84 & 54 & 64 & \\
\hline & total: & & & 169 & 169 & 169 & 106 & 63 & \\
\hline \multirow{3}{*}{$\begin{array}{l}(11) \\
\text { Jansen } \\
2011\end{array}$} & $\begin{array}{l}\text { I: parent } \\
\text { CBT }\end{array}$ & - & 161 & 59 & - & 54 & 54 & 92 & \multirow{3}{*}{$\begin{array}{l}12 \text { weeks } \\
\text { post } 12- \\
\text { week inter- } \\
\text { vention ( } 24 \\
\text { weeks) }\end{array}$} \\
\hline & $\begin{array}{l}\text { C: waiting } \\
\text { list control }\end{array}$ & & & 39 & - & 34 & 34 & 87 & \\
\hline & total: & & & 98 & & 88 & 88 & 90 & \\
\hline \multirow[t]{4}{*}{$\begin{array}{l}(10) \\
\text { Collins } \\
2011\end{array}$} & $\begin{array}{l}\text { I: parent- } \\
\text { only - diet }\end{array}$ & \multirow{3}{*}{$\begin{array}{l}\text { Power: } \\
80 \% \\
\text { chance of } \\
\text { detect- } \\
\text { ing signif- } \\
\text { icance (2- } \\
\text { sided } 5 \% \\
\text { level), with } \\
\text { a } \\
0.26 \text { BMI } \\
\text { z score dif- } \\
\text { ference } \\
\text { from base- } \\
\text { line to } 12 \\
\text { months as } \\
\text { the initial } \\
\text { end point, } \\
\text { with an an- } \\
\text { tic- } \\
\text { ipated loss }\end{array}$} & \multirow[t]{3}{*}{$505 / 319$} & 63 & - & 42 & 22 & 35 & \multirow[t]{4}{*}{$\begin{array}{l}80 \text { weeks } \\
\text { post 24- } \\
\text { week inter- } \\
\text { vention } \\
(104 \\
\text { weeks) }\end{array}$} \\
\hline & $\begin{array}{l}\mathrm{C} 1 \text { : par- } \\
\text { ent-child } \\
\text { (physical } \\
\text { activity) }\end{array}$ & & & 73 & - & 63 & 35 & 48 & \\
\hline & $\begin{array}{l}\text { C2: par- } \\
\text { ent-child } \\
\text { (phys- } \\
\text { ical activity } \\
+ \text { diet) }\end{array}$ & & & 70 & - & 60 & 36 & 51 & \\
\hline & \multicolumn{3}{|l|}{ total: } & 206 & - & 165 & 93 & 45 & \\
\hline $\begin{array}{l}(9) \\
\text { Boutelle } \\
2011\end{array}$ & $\begin{array}{l}\text { I: parent- } \\
\text { only }\end{array}$ & $\begin{array}{l}\text { Sample } \\
\text { size was } \\
\text { deter- } \\
\text { mined by } \\
\text { pragmatic } \\
\text { factors, } \\
\text { including } \\
\text { budget } \\
\text { and in- } \\
\text { vestigator } \\
\text { time com- } \\
\text { mitments. } \\
\text { No interim }\end{array}$ & 157 & 40 & - & 24 & 24 & 60 & $\begin{array}{l}24 \text { weeks } \\
\text { post 20- } \\
\text { week inter- } \\
\text { vention } \\
\text { (week 44) }\end{array}$ \\
\hline
\end{tabular}


Table 1. Overview of study populations (Continued)

$$
\begin{aligned}
& \text { analyses } \\
& \text { were } \\
& \text { done. The } \\
& \text { hypotheses } \\
& \text { tested } \\
& \text { related } \\
& \text { to non- } \\
& \text { inferiority } \\
& \text { of the } \\
& \text { parent } \\
& \text { treatment } \\
& \text { to the par- } \\
& \text { ent-child } \\
& \text { treatment } \\
& \text { on child } \\
& \text { and parent } \\
& \text { weight } \\
& \text { loss and } \\
& \text { child daily } \\
& \text { caloric } \\
& \text { intake and } \\
& \text { physical } \\
& \text { activity. } \\
& \text { The bound } \\
& \text { for non- } \\
& \text { inferiority } \\
& \text { hypotheses } \\
& \text { related } \\
& \text { to BMI } \\
& \text { percentile } \\
& \text { was set to } \\
& 1 . \quad \text { This } \\
& \text { is the } \\
& \text { maximum } \\
& \text { value the } \\
& \text { parent- } \\
& \text { child } \\
& \text { group } \\
& \text { could dow } \\
& \text { better than } \\
& \text { parent- } \\
& \text { only, be- } \\
& \text { This } \\
& \text { noncluded. } \\
&
\end{aligned}
$$


Table 1. Overview of study populations (Continued)

$$
\begin{aligned}
& \text { bound } \\
& \text { could cor- } \\
& \text { respond to } \\
& \text { an mean- } \\
& \text { aged child } \\
& \text { in this } \\
& \text { sample } \\
& \text { having a } \\
& \text { BMI of } \\
& 26 \text { in the } \\
& \text { parent- } \\
& \text { child } \\
& \text { group and } \\
& 28.5 \quad \text { in } \\
& \text { the parent- } \\
& \text { only group } \\
& \text { at post- } \\
& \text { treatment/ } \\
& \text { follow-up, } \\
& \text { assuming } \\
& \text { equiva- } \\
& \text { lence at } \\
& \text { baseline. } \\
& \text { For a non- } \\
& \text { inferiority } \\
& \text { bound for } \\
& \text { child BMI, } \\
& \text { which was } \\
& \text { selected } \\
& \text { post hoc, } \\
& \text { we con- } \\
& \text { sidered } \\
& \text { value of } \\
& \text { BMI = } 1 \\
& \text { BMoosing a } \\
& \text { ins that } \\
& \text { would cor- } \\
& \text { respond to } \\
& \text { the BMI } \\
& \text { percentile } \\
& \text { non- } \\
& \text { inferiority } \\
& \text { bound } \\
& \text { (BMI }
\end{aligned}
$$


Table 1. Overview of study populations (Continued)

\begin{tabular}{|c|c|c|c|c|c|c|c|c|c|}
\hline & $\begin{array}{l}\text { C: parent- } \\
\text { child }\end{array}$ & & & 40 & - & 28 & 28 & 70 & \\
\hline & total: & & & 80 & & 52 & 52 & 65 & \\
\hline \multirow[t]{3}{*}{$\begin{array}{l}(8) \text { West } \\
2010\end{array}$} & $\begin{array}{l}\text { I: parent- } \\
\text { only }\end{array}$ & - & 205 & 52 & 52 & 52 & 34 & 65 & \multirow{3}{*}{$\begin{array}{l}40 \text { weeks } \\
\text { post } 12- \\
\text { week inter- } \\
\text { vention ( } 52 \\
\text { weeks) }\end{array}$} \\
\hline & $\begin{array}{l}\text { C: waiting } \\
\text { list control }\end{array}$ & & & 49 & 49 & 49 & 46 & 94 & \\
\hline & total: & & & 101 & 101 & 101 & 80 & 79 & \\
\hline \multirow[t]{3}{*}{$\begin{array}{l}(7) \\
\text { Resnick } \\
2009\end{array}$} & $\begin{array}{l}\text { I: educa- } \\
\text { tional ma- } \\
\text { terial } \\
+ \text { personal } \\
\text { encounters }\end{array}$ & - & $84 / 46$ & 22 & - & 18 & 18 & 82 & \multirow[t]{3}{*}{$\begin{array}{l}\text { Unclear } \\
\text { ( } 41 \text { weeks } \\
\text { be- } \\
\text { tween start } \\
\text { and last } \\
\text { mail out) }\end{array}$} \\
\hline & $\begin{array}{l}\text { C: educa- } \\
\text { tional ma- } \\
\text { terial }\end{array}$ & & & 24 & - & 24 & 24 & 100 & \\
\hline & total: & & & 46 & & 42 & 42 & 91 & \\
\hline $\begin{array}{l}(6) \quad \text { Es- } \\
\text { tabrooks } \\
2009\end{array}$ & $\begin{array}{l}\text { I1: parent } \\
\text { group } \quad+ \\
\text { IVR }\end{array}$ & $\begin{array}{l}\text { Sam- } \\
\text { ple size cal- } \\
\text { culations } \\
\text { were com- } \\
\text { pleted, } \\
\text { varying the } \\
\text { detectable } \\
\text { effect sizes } \\
\text { from small } \\
\text { to medium } \\
\text { with a } \\
\text { power of } 0 \text {. } \\
8 \text {. The re- } \\
\text { sult was a } \\
\text { need for } 42 \\
\text { partic- } \\
\text { ipants per } \\
\text { interven- } \\
\text { tion to de- } \\
\text { tect } \\
\text { a medium } \\
\text { effect and } \\
64 \text { partici- } \\
\text { pants }\end{array}$ & $1487 / 656$ & 85 & - & 63 & 63 & 74 & $\begin{array}{l}28-40 \\
\text { weeks post } \\
12-\text { to } 24- \\
\text { week inter- } \\
\text { vention ( } 52 \\
\text { weeks) }\end{array}$ \\
\hline
\end{tabular}


Table 1. Overview of study populations (Continued)

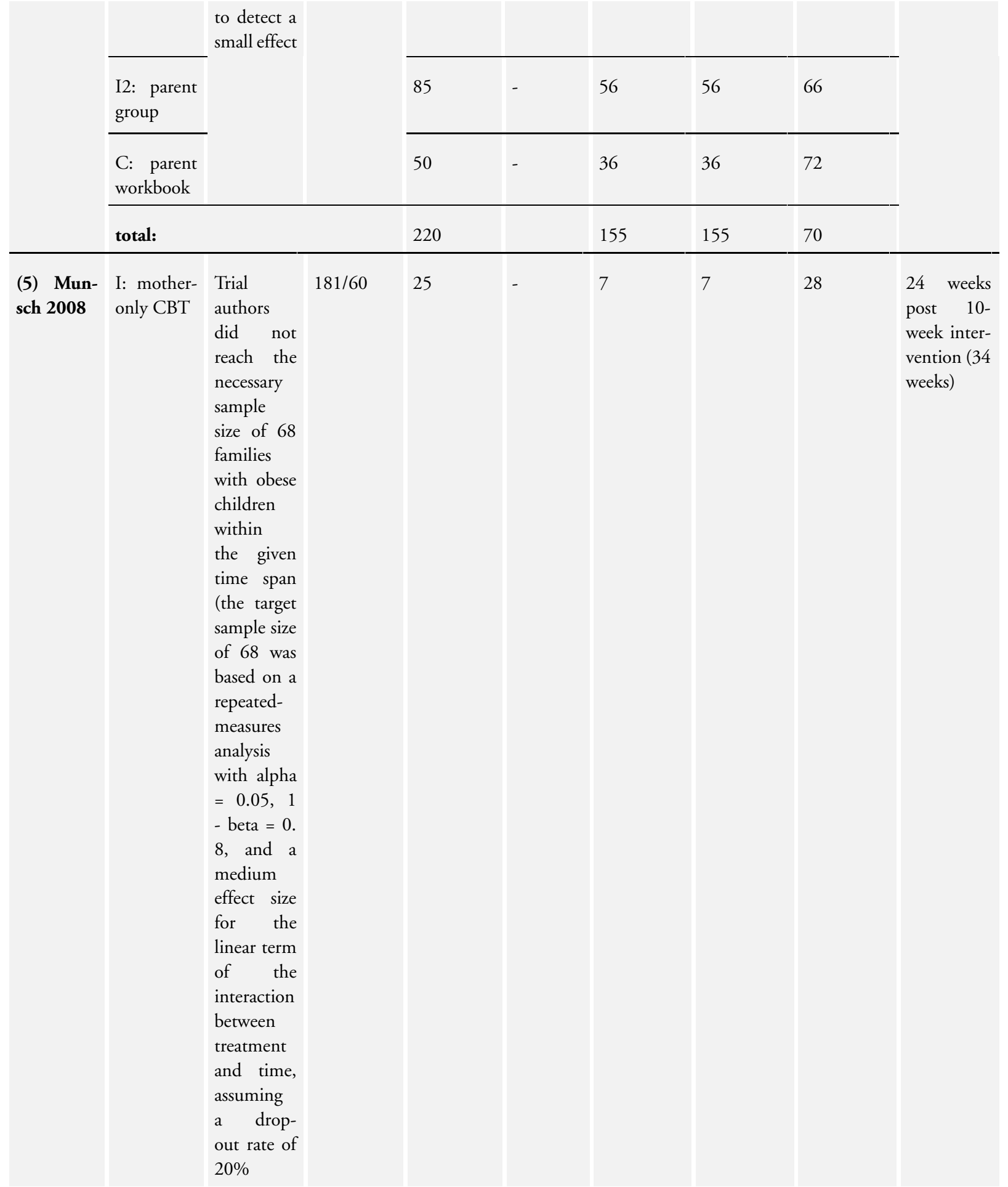


Table 1. Overview of study populations (Continued)

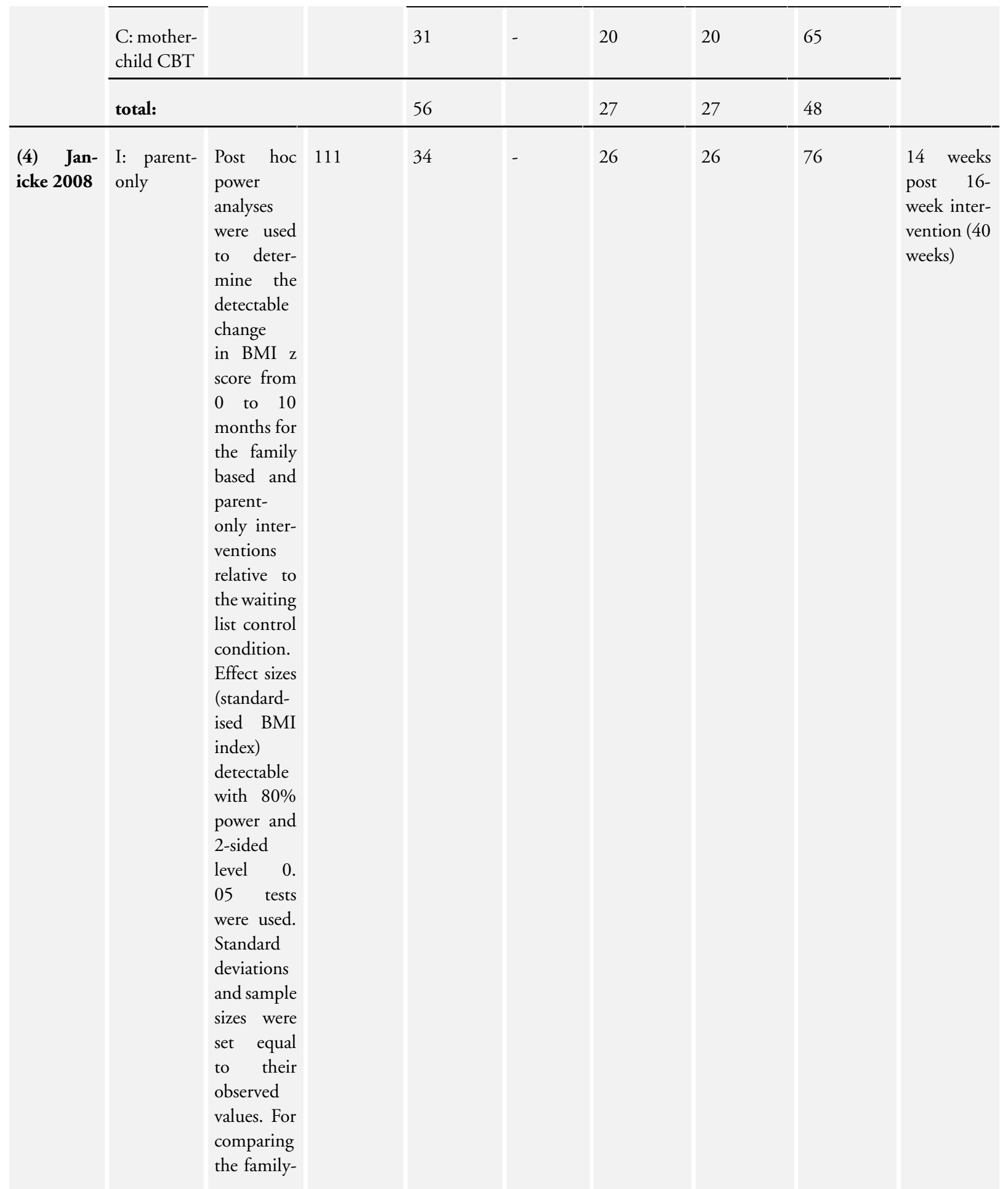


Table 1. Overview of study populations (Continued)

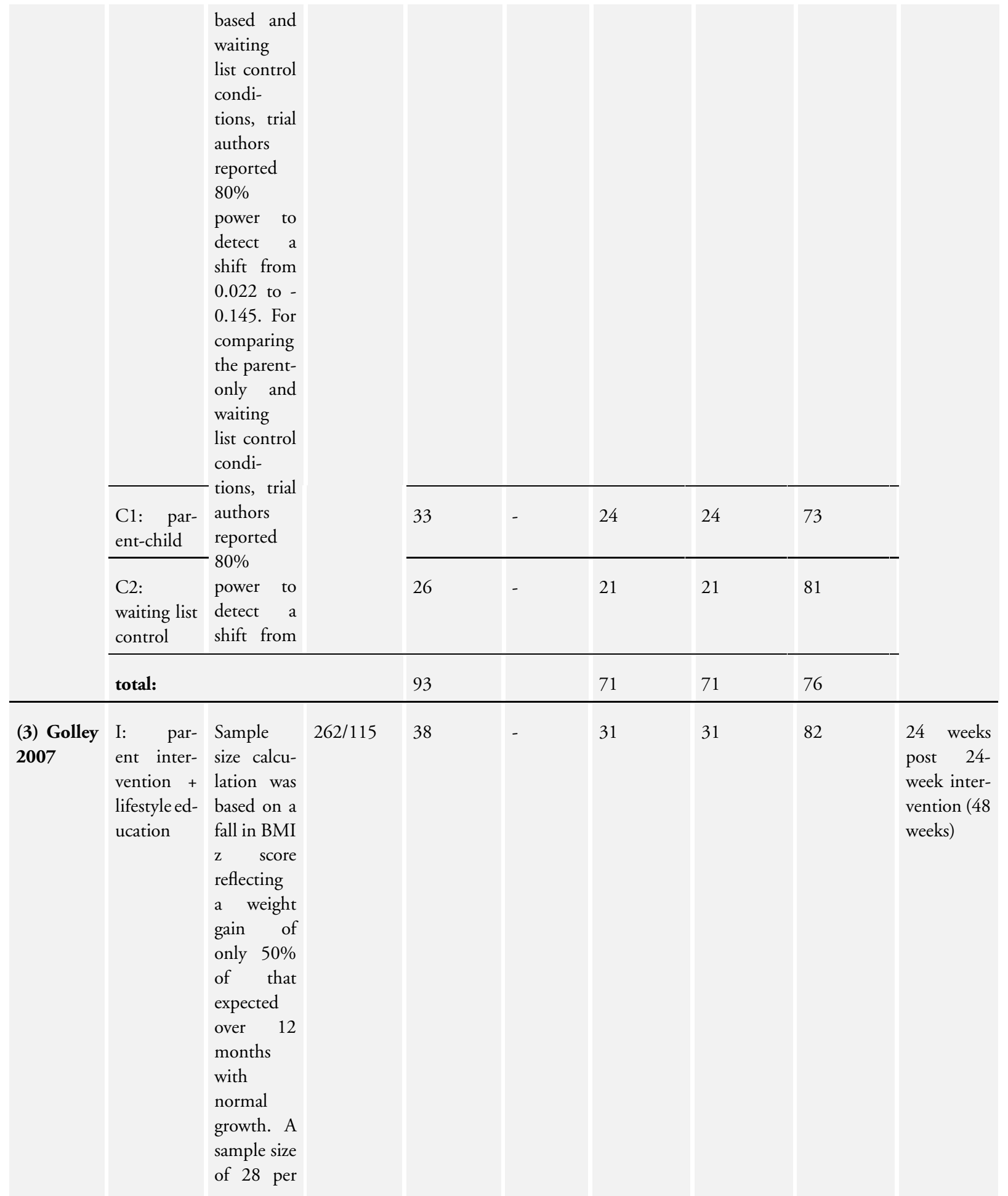


Table 1. Overview of study populations (Continued)

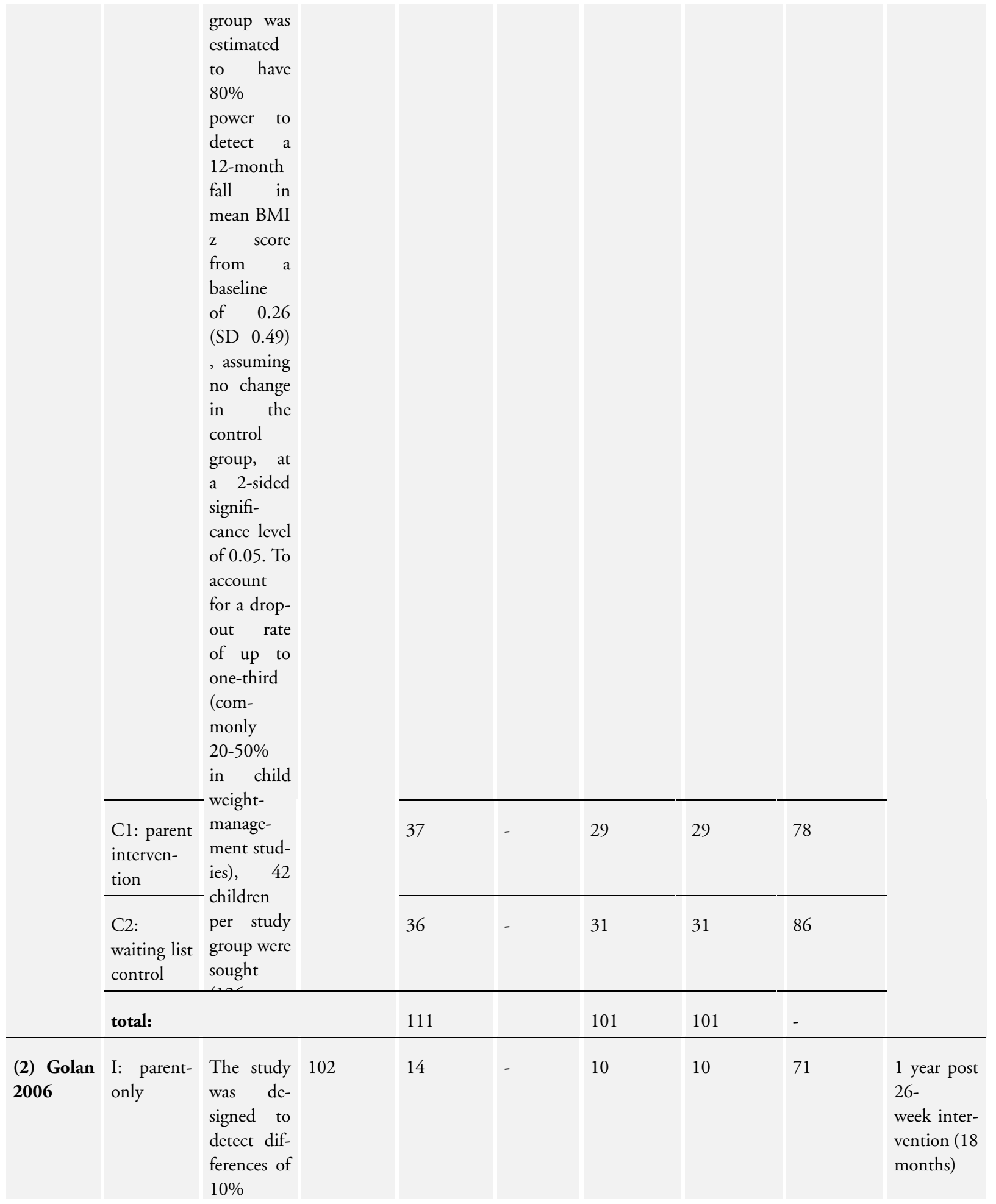


Table 1. Overview of study populations (Continued)

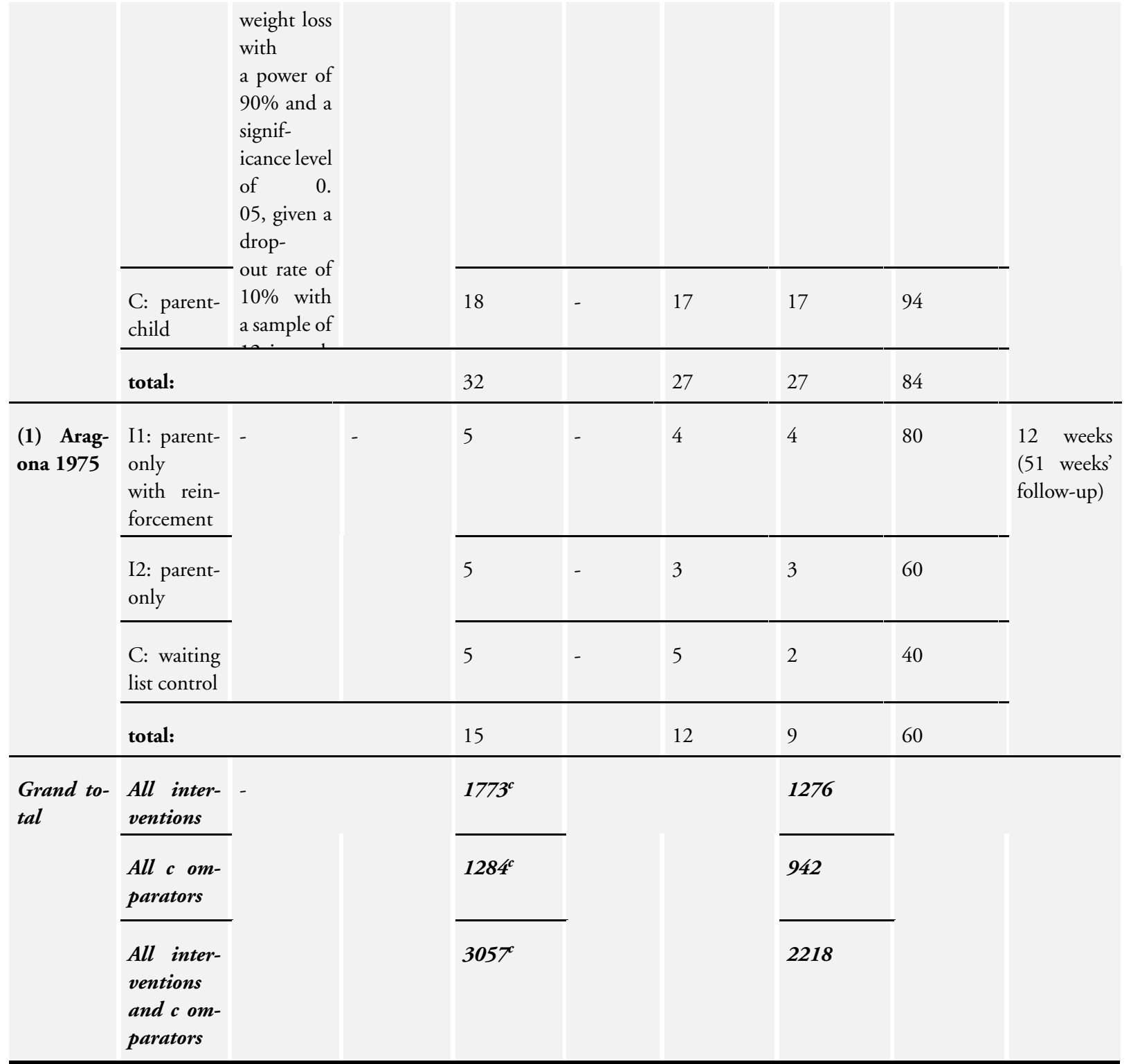

\footnotetext{
${ }^{a}$ According to power calculation in trial publication or report

${ }^{b}$ Duration of intervention or follow-up (or both) under randomised conditions until end of trial

${ }^{c}$ Some trials had more than one intervention/comparator group

“-” denotes not reported

BMI: body mass index; C: comparator; CBT: cognitive behavioural therapy; I: intervention; ITT: intention-to-treat; IVR: interactive voice response; $\mathrm{n}$ : number of participants; NOURISH: nourishing our understanding of role modelling to improve support and health; PCP: primary care providers; SD: standard deviation; YHC: Youth Health Care
} 
A P P E N DICES

\section{Appendix I. Search strategies}

\section{Cochrane Library}

Part I: Obesity

1. [mh Ôbesity]

2. [mh “"Obesity, Morbid”]

3. [mh “"Obesity, Abdominal”]

4. [mh "Pediatric Obesity"]

5. [mh Ôverweight]

6. [mh “"Weight Loss"]

7. (adipos* or obes*):ti,ab

8. (overweight* or ("over" next weight $\left.{ }^{*}\right)$ ):ti,ab

9. ("weight" near/1 (reduc* or los* or control* or manage*)):ti,ab

10. $\{$ or \#1-\#9\}

Part II: Intervention

11. [mh "Behavior Therapy"]

12. [mh "Counseling"]

13. [mh “"Family Therapy"]

14. [mh “"Social Support"]

15. [mh “"Program Evaluation"]

16. [mh "Exercise"]

17. [mh "Exercise Therapy"]

18. [mh "Physical Education and Training"]

19. [mh "Exercise Movement Techniques"]

20. [mh "Motor Activity"]

21. [mh Diet]

22. [mh "Diet Therapy"]

23. [mh "Patient Education as Topic"]

24. [mh "Health Education"]

25. [mh "Health Behavior"]

26. [mh "Health Promotion"]

27. [mh "School Health Services"]

28. [mh “"School Nursing"]

29. [mh “"Life style"]

30. (("obesity" near/4 “intervention") or "program" or "programme" or "camp" or "camps"):ti,ab

31. ("lifestyle" or "life style"):ti,ab

32. exercis*:ti,ab

33. (physic* next (activ* or fit*)):ti,ab

34. (walk* or jog* or swim* or ("weight" next lift*) or danc* or "aerobics"):ti,ab

35. ((physic* or strength* or resist* or "circuit" or "weight" or aerob* or "cross" or "endurance" or structur*) near/4 train*):ti,ab

36. ("behavioral" or "behavioural" or (("behavior" or "behaviour") next "modification") or psychoth* or "psychosocial"):ti,ab

37. (("group" or "family" or cognit* or behav*) next therap*):ti,ab

38. (counselling or counselling):ti,ab

39. educat*:ti,ab

40. (("parent" or "parents" or "family") next ("based" or "focused" or "directed" or "centered" or "only" or "led")):ti,ab

41. (diet* or "healthy nutrition" or (nutrition* next ("knowledge" or educat* or therap* or program* or intervention*))):ti,ab 
42. $\{$ or \#11-\#41\}

Part III: Part I + Part II and additional MeSH/subheading combination

43. \#10 and \#42

44. [mh Ôbesity] or [mh “"Obesity, Morbid”] or [mh Ôverweight]

45. [mh /DH,PC,RH,TH,PX] [diet therapy or prevention \& control or rehabilitation or therapy or psychology]

46. \#44 and \#45

47. \#43 or \#46

Part IV: Population [adapted from Leclercq 2013]

48. [mh Âdolescent]

49. [mh Child]

50. [mh Înfant]

51. [mh ^Pediatrics]

52. "minors": $t i, a b$

53. ("boy" or "boys" or "boyhood"):ti,ab

54. girl*:ti,ab

55. ("kid" or "kids"):ti,ab

56. infant*:ti,ab

57. ("baby" or "babies"):ti,ab

58. ("toddler" or "toddlers"):ti,ab

59. ("child" or "childs" or children* or childhood* or childcare* or schoolchild*):ti,ab

60. adolescen*:ti,ab

61. juvenil*:ti,ab

62. youth*:ti,ab

63. (teen* or preteen*):ti,ab

64. (underage* or (“under" next age*)):ti,ab

65. pubescen*:ti,ab

66. (paediatric* or paediatric*):ti,ab

67. \{or \#48-\#66\}

Part V: Part III AND IV and additional MeSH/subheading combination

68. \#47 and \#67

69. [mh “"Pediatric Obesity"]

70. [mh /DH,PC,RH,TH,PX]

71. \#69 and \#70

72. \#68 or \#71

\section{MEDLINE (Ovid SP)}

Part I: Obesity

1. Obesity/

2. Obesity, Morbid/

3. Obesity, Abdominal/

4. Pediatric Obesity/

5. Overweight/

6. Weight Loss/

7. (adipos* or obes*).tw.

8. (overweight* or over weight*).tw.

9. (weight adj1 (reduc* or los* or control* or manage*)).tw.

10. or/1-9

Part II: Intervention

11. exp Behavior Therapy/ 


\section{(Continued)}

12. exp Counseling/

13. Family Therapy/

14. Social Support/

15. Program Evaluation/

16. exp Exercise/

17. exp Exercise Therapy/

18. exp "Physical Education and Training"/

19. exp Exercise Movement Techniques/

20. Motor Activity/

21. exp Diet/

22. exp Diet Therapy/

23. Patient Education as Topic/

24. Health Education/

25. exp Health Behavior/

26. exp Health Promotion/

27. School Health Services/

28. School Nursing/

29. Life style/

30. ((obesity adj3 intervention) or program or programme or camp?).tw

31. (lifestyle or life style).tw.

32. exercis*.tw.

33. (physic* adj (activ* or fit*)).tw.

34. (walk* or jog* or swim* or weight lift* or danc* or aerobics).tw

35. ((physic* or strength* or resist* or circuit or weight or aerob* or cross or endurance or structur*) adj3 train*).tw

36. (behavio?ral or behavio?r modification or psychoth* or psychosocial).tw

37. ((group or family or cognit* or behav*) adj therap*).tw.

38. counsel?ing.tw.

39. educat*.tw.

40. ((parent? or family) adj (based or focused or directed or centered or only or led)).tw

41. (diet* or healthy nutrition or (nutrition* adj (knowledge or educat* or therap* ${ }^{*}$ or program* or intervention*))).tw

42. or/11-41

Part III: Part I + Part II and additional MeSH/subheading combination

43. 10 and 42

44. Obesity/ or Obesity, Morbid/ or Overweight/ or Weight Loss/

45. diet therapy.fs. or prevention \& control.fs. or rehabilitation.fs. or therapy.fs. or psychology.fs

46. 44 and 45

47. 43 or 46

Part IV: Population [adapted from Leclercq 2013]

48. Adolescent/

49. exp Child/

50. Infant/

51. Pediatrics/

52. minors.tw.

53. (boy or boys or boyhood).tw.

54. girl*.tw.

55. infant*.tw.

56. (baby or babies).tw.

57. toddler?.tw.

58. (kid or kids).tw. 


\section{(Continued)}

59. (child or childs or children* or childhood* ${ }^{*}$ or childcare* or schoolchild*).tw

60. adolescen*.tw.

61. juvenil*.tw.

62. youth*.tw.

63. (teen* ${ }^{*}$ or preteen*).tw.

64. (underage* or under age*).tw.

65. pubescen*.tw.

66. p?ediatric*.tw.

67. or/48-66

Part V: Part III AND IV and additional MeSH/subheading combination

68. 47 and 67

69. Pediatric Obesity/

70. diet therapy.fs. or prevention $\&$ control.fs. or rehabilitation.fs. or therapy.fs. or psychology.fs

71.69 and 70

72.68 or 71

Part VI: Study filter [Cochrane Handbook 2008 RCT filter - sensitivity and precision maximizing version]

73. randomised controlled trial.pt.

74. controlled clinical trial.pt.

75. randomi?ed.ab.

76. placebo.ab.

77. clinical trials as topic/

78. randomly.ab.

79. trial.ti.

80. or/73-79

81. exp animals/ not humans/

82. 80 not 81

Part VII: Part V+ Part VI

83. 72 and 82

\section{EMBASE (Ovid SP)}

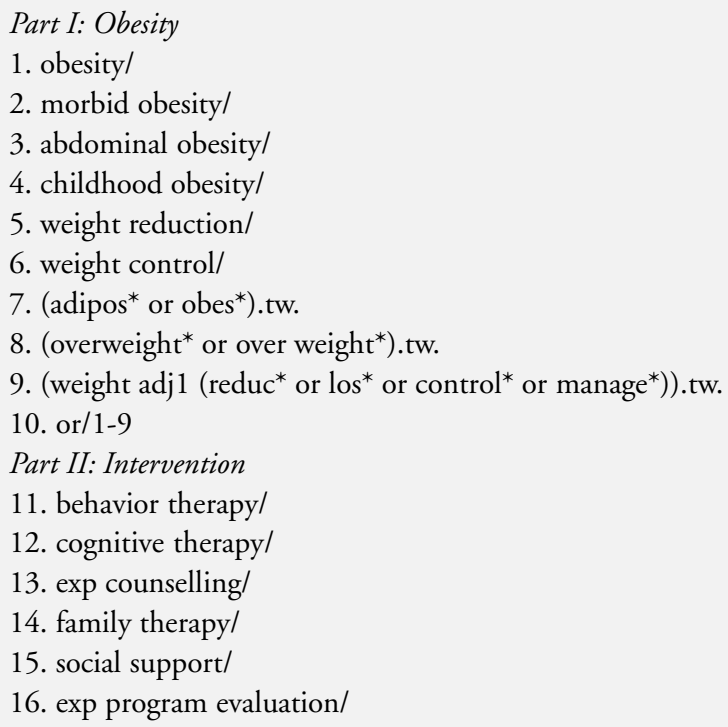




\section{(Continued)}

17. exp exercise/

18. exp physical education/

19. exp physical activity/

20. exp motor activity/

21. training/

22. exp diet/

23. exp diet therapy/

24. nutritional health/

25 . child nutrition/

26. feeding behavior/

27. patient education/

28 . health promotion/

29. health literacy/

30. nutrition education/

31. health education/

32. school health education/

33. school health service/

34. lifestyle/

35. lifestyle modification/

36. ((obesity adj3 intervention) or program or programme or camp?).tw

37. (lifestyle or life style).tw.

38. exercis*.tw.

39. (physic* adj (activ* or fit $\left.{ }^{*}\right)$ ).tw.

40. (walk* or jog* or swim* or weight lift* or danc* or aerobics).tw

41. ((physic* or strength* or resist* or circuit or weight or aerob* or cross or endurance or structur*) adj3 train*).tw

42. (behavio?ral or behavio?r modification or psychoth* or psychosocial).tw

43. ((group or family or cognit* or behav*) adj therap*).tw.

44. counsel?ing.tw.

45. educat*.tw.

46. ((parent? or family) adj (based or focused or directed or centered or only or led)).tw

47. (diet* or healthy nutrition or (nutrition* adj (knowledge or educat* or therap* or program* or intervention*))).tw 48. or/11-47

Part III: Part I + Part II and additional MeSH/subheading combination

49. 10 and 48

50. obesity/ or morbid obesity/

51. pc.fs or rh.fs or th.fs. [prevention.fs. or rehabilitation.fs. or therapy.fs.]

52.50 and 51

53. 49 or 52

Part IV: Population [adapted from Leclercq 2013]

54. juvenile/

55. adolescent/

56. child/

57. infant/

58. baby/

59. toddler/

60. preschool child/

61. school child/

62. pediatrics/

63. minors.tw. 
64. (boy or boys or boyhood).tw.

65. girl*.tw.

66. infant*.tw.

67. (baby or babies).tw.

68. toddler?.tw.

69. (kid or kids).tw.

70. (child or childs or children* or childhood* or childcare* or schoolchild*).tw

71. adolescen*.tw.

72. juvenil*.tw.

73. youth*.tw.

74. (teen* or preteen*).tw.

75. (underage* or under age*).tw.

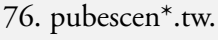

77. p?ediatric*.tw.

78. or/54-77

Part V: Part III AND IV and additional MeSH/subheading combination

79. 53 and 78

80. childhood obesity/

81. pc.fs or rh.fs or th.fs. [prevention.fs. or rehabilitation.fs. or therapy.fs.]

82. 80 and 81

83. 79 or 82

Part VI: Study filter [Wong 2006afilter - SDSSGS version]

84. random*.tw. or clinical trial*.mp. or exp treatment outcome/

Part VII: Part V + Part VI

85.83 and 84

\section{PsycINFO (Ovid SP)}

Part I: Obesity

1. exp Overweight

2. (adipos* or obes*).tw.

3. (overweight* or over weight*).tw.

4. or/1-3

Part II: Intervention

5. Weight Control/

6. Weight Loss/

7. Aerobic Exercise/

8. Diets/

9. exp Exercise/

10. Movement Therapy/

11. Dance Therapy/

12. exp Physical Activity/

13. Physical Fitness/

14. Health Behavior/

15. Health Promotion/

16. Health Knowledge/

17. Health Literacy/

18. Health Education/

19. Client Education/ 


\section{(Continued)}

\section{Lifestyle/}

21. Physical Education/

22. exp Program Evaluation/

23. Educational Programs/

24. Educational Therapy/

25. exp Program Development/

26. School Based Intervention/

27. School Counseling/

28. Counseling/

29. Group Counseling/

30. Family Therapy/

31. Support Groups/

32. Social Support/

33. School Counselors/

34. exp Behavior Modification/

35. Cognitive Behavior Therapy/

36. Cognitive Therapy/

37. ((obesity adj3 intervention) or program or programme or camp?).tw

38. (lifestyle or life style).tw.

39. exercis*.tw.

40. (physic* adj (activ* or fit*)).tw.

41. (walk* or jog* or swim* or weight lift* or danc* or aerobics).tw

42. ((physic* or strength* or resist* or circuit or weight or aerob* or cross or endurance or structur*) adj3 train*).tw

43. (behavio?ral or behavio?r modification or psychoth* or psychosocial).tw

44. ((group or family or cognit* or behav*) adj therap*).tw.

45. counsel?ing.tw.

46. educat*.tw.

47. ((parent? or family) adj (based or focused or directed or centered or only or led)).tw

48. (diet* or healthy nutrition or (nutrition* adj (knowledge or educat* or therap* or program* or intervention*))).tw 49. or/5-48

Part III: Part I + Part II

50. 4 and 49

Part IV: Population [adapted from Leclercq 2013]

51. minors.tw.

52. (boy or boys or boyhood).tw.

53. girl*.tw.

54. infant*.tw.

55. (baby or babies).tw.

56. toddler?.tw.

57. (kid or kids).tw.

58. (child or childs or children* or childhood* or childcare* or schoolchild*).tw

59. adolescen*.tw.

60. juvenil*.tw.

61. youth*.tw.

62. (teen* or preteen*).tw.

63. (underage* or under age*).tw.

64. pubescen*.tw.

65. p?ediatric*.tw.

66. or/51-65 
Part V: Part III AND IV and additional MeSH/subheading combination

67.50 and 66

Part VI: Study filter [ Eady 2008filter - BS version]

68. control*.tw. OR random*.tw. OR exp Treatment/

Part VII: Part V + Part VI

69. 67 and 68

\section{CINAHL (EBSCOhost)}

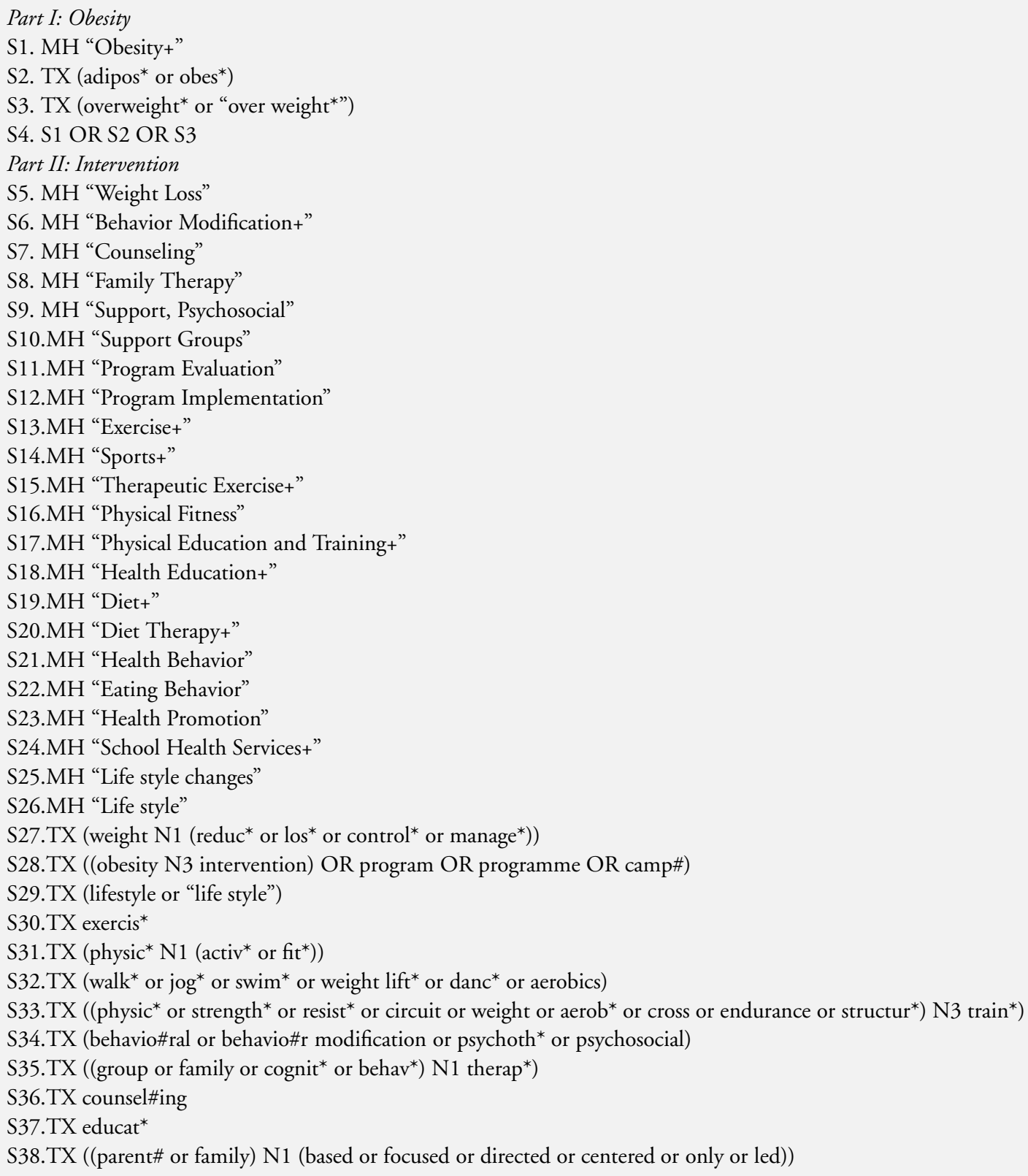

Parent-only interventions for childhood overweight or obesity in children aged 5 to I I years (Review)

Copyright @ 2015 The Cochrane Collaboration. Published by John Wiley \& Sons, Ltd. 
S39.TX (diet* or "healthy nutrition" or (nutrition* N1 (knowledge or educat* or therap* or program* or intervention*))) S40.S5 OR S6 OR S7 OR S8 OR S9 OR S10 OR S11 OR S12 OR S13 OR S14 OR S15 OR S16 OR S17 OR S18 OR S19 OR S20 OR S21 OR S22 OR S23 OR S24 OR S25 OR S26 OR S27 OR S28 OR S29 OR S 30 OR S31 OR S32 OR S33 OR S34 OR S35 OR S36 OR S37 OR S38 OR S39

Part III: Part I + Part II and additional MeSH/subheading combination

S41.S4 AND S40

S42.(MH "Obesity+/DH/ED/PC/PF/RH/TH") [diet therapy or education or prevention \& control or psychosocial factors or rehabilitation or therapy]

S43.S41 OR S42

Part IV: Population [based on Leclercq 2013]

S44.MH "Adolescence"

S45.MH "Child+"

S46.MH "Infant"

S47.MH "Pediatrics"

S48.TX minors

S49.TX (boy OR boys OR boyhood)

S50.TX girl*

S51.TX infant*

S52.TX (baby OR babies)

S53.TX toddler\#

S54.TX (kid OR kids)

S55.TX (child OR childs OR children* OR childhood* OR childcare* OR schoolchild*)

S56.TX adolescen*

S57.TX juvenil*

S58.TX youth*

S59.TX (teen* or preteen*)

S60.TX (underage* or under age*)

S61.TX pubescen*

S62.TX (paediatric* OR paediatric*)

S63.S44 OR S45 OR S46 OR S47 OR S48 OR S49 OR S50 OR S51 OR S52 OR S53 OR S54 OR S55 OR S56 OR S57 OR S58 OR S59 OR S60 OR S62

Part V: Part III AND IV and additional MeSH/subheading combination

S64.S43 AND S63

S65.(MH "Pediatric Obesity/DH/ED/PC/PF/RH/TH") [diet therapy or education or prevention \& control or psychosocial factors or rehabilitation or therapy]

S66.S64 OR S65

Part VI: Study filter [ Wong 2006bfilter - SDSSGS version]

S67.MH "treatment outcomes+" OR MH "experimental studies+" or random*

Part VII: Part V+ Part VI

S68.S66 AND S67

\section{LILACS (IAHx)}

((((MH: “Obesity” OR MH:“Obesity, Morbid” OR MH:“Obesity, Abdominal” OR MH: "Pediatric Obesity” OR MH: "Overweight” OR adipos\$ OR obes\$ OR overweight\$ OR “over weight” OR sobrepes\$ OR “exceso de peso" OR “excesso de peso”) AND (MH: "Weight Loss" OR MH:"Exercise” OR MH:"Exercise Therapy” OR MH:"Physical Education and Training" OR MH:"Exercise Movement Techniques" OR MH: "Weight Reduction Programs" OR MH:"Motor Activity” OR MH: "Behavior Therapy" OR MH: "Counseling” OR MH:"Family Therapy” OR MH:“Social Support” OR MH:"Program Evaluation” OR MH:"Diet” OR MH: "Diet Therapy" OR MH:"Patient Education as Topic" OR MH:"Health Education” OR MH:"Health Behavior" OR MH:"Health Promotion” OR MH:“Weight Reduction Programs” OR MH:“School Health Services” OR MH:“Life style” OR exerci\$ OR ejerci\$

Parent-only interventions for childhood overweight or obesity in children aged 5 to II years (Review)

Copyright @ 2015 The Cochrane Collaboration. Published by John Wiley \& Sons, Ltd. 
OR ((physic\$ OR fisic\$) AND (activ\$ OR ativid\$ OR fit\$ OR educac\$ OR entrenam\$ OR treinam\$)) OR ((physic\$ OR fisic\$ OR strength\$ OR forca OR fuerza OR resist\$ OR circuit\$ OR weight OR aerob\$ OR endurance OR structur\$ OR estructur\$) AND train\$ OR treina\$ OR entrena\$) OR program\$ OR “estilo de vida” OR padres OR pais OR familia OR familias OR familiar OR terapia OR orienta\$ OR educa\$ OR diet\$ OR nutric\$ OR “weight reduction” OR "weight loss" OR "weight control” OR "control de peso")) OR (MH:“Obesity/diet therapy” OR MH: "Obesity, Morbid/diet therapy” OR MH: "Overweight/diet therapy” OR MH: "Obesity/prevention \& control” OR MH: “Obesity, Morbid/prevention \& control ” OR MH: "Overweight/prevention \& control” OR MH:“Obesity/rehabilitation” OR MH:“Obesity, Morbid/rehabilitation” OR MH: "Overweight/rehabilitation” OR MH: "Obesity/ therapy” OR MH:“Obesity, Morbid/therapy” OR MH:“Overweight/therapy” OR MH:“Obesity/psychology” OR MH:“Obesity, Morbid/psychology” OR MH: “Overweight/psychology”)) AND (MH:“Adolescent” OR MH: "Child” OR MH:"Pediatrics” OR MH: "Infant” OR minors OR boy OR boys OR girl\$ OR kid OR kids OR child OR childs OR children\$ OR childhood\$ OR childcare\$ OR schoolchild\$ OR escolar\$ OR adolescen\$ OR preadolescen\$ OR juvenil\$ OR juventud\$ OR youth\$ OR teen\$ OR preteen\$ OR underage\$ OR pubescen\$ OR paediatri\$ OR pediatri\$ OR joven\$ OR jovem\$ OR niños OR niñas OR crianca\$ OR menin\$ OR "menor de edad" OR "menores de edad" OR "menor de idade" OR "menores de idade")) OR MH: "Pediatric Obesity/diet therapy" OR MH: "Pediatric Obesity/prevention \& control” OR MH: "Pediatric Obesity/rehabilitation” OR MH: "Pediatric Obesity/therapy” OR MH:"Pediatric Obesity/psychology" [activated filter "Controlled Clinical Trial"]

ICTRP Search Portal (Advanced search)

[activated "Search for clinical trials in children"]:

in Title: obes* OR overweight*

OR

in Condition: obes* OR overweight*

Recruitment Status: ALL

ClinicalTrials.gov (Advanced search)

Conditions: obese OR overweight OR obesity

Study type: Interventional Studies

Age Group: Child (birth-17)

\section{Appendix 2. Description of interventions}

\begin{tabular}{l|l|l|}
\hline & Intervention(s) & Comparator(s) \\
\hline Resnicow 2015 & $\begin{array}{l}\text { Parent-only PCP motivational interviewing: } 4 \text { ses- } \\
\text { sions over 2 years with the primary care provider and } \\
\text { standard advice on healthy eating and exercise }\end{array}$ & $\begin{array}{l}\text { Usual care: standard advice on healthy eating and ex- } \\
\text { reise }\end{array}$ \\
\cline { 2 - 3 } & $\begin{array}{l}\text { Parent-only PCP + dietician motivational inter- } \\
\text { viewing: } 4 \text { sessions over 2 years with the primary care } \\
\text { provider and } 6 \text { sessions with a dietician. Standard ad- } \\
\text { vice on healthy eating and exercise }\end{array}$ \\
\hline
\end{tabular}

Parent-only interventions for childhood overweight or obesity in children aged 5 to I I years (Review) 
Mazzeo 2014 Parent NOURISH: parents' self efficacy to make positive changes in eating and exercise behaviours
Parent control: single session on diet and exercise and mailed out information van Grieken 2013 Parent-only: healthy lifestyle counselling at well-child visit and up to 3 additional sessions at 3, 6 and 12 months, included motivational interviewing; focus on targeting key lifestyle-related behaviours
Usual care control: general information about healthy lifestyle
Parent-only: focus on healthy habits in young children, nutritional information, information regarding increasing physical activity
Parent control: provision of educational age-appropriate, evidence-based health and safety information and group sessions
Esfarjani 2013

Parent-only: educational sessions on nutrition, physical activity and behavioural control
Moens 2012

Parent-only: 5-month intervention, 6 group meetings of 2 hours, focused on education of dietary requirements and training for parenting behavioural skills
Parent control: 2 parent training sessions

Waiting list control: for 6 months
Parent-only: included behavioural strategies focused on increasing child growth monitoring and providing feedback to families
(I2) C1: parent intervention diet 'decrease': parent intervention as in parent-only intervention and children and parents intervention focusing on decreasing sugar-sweetened beverage and sweet and salty snack food intake

(I3) C2: parent intervention diet 'increase': parent intervention as in parent-only intervention and children and parents intervention focusing on increasing fruit, vegetable and low-fat dairy intake

\section{Raynor 2012b}

Parent-only: included behavioural strategies focused on increasing child growth monitoring and providing feedback to families
(I2) C1: parent intervention diet and activity 'traditional': parent intervention as in parent-only intervention and children and parents intervention focusing on decreasing sugar-sweetened beverage intake and increasing physical activity

(I3) C2: parent intervention diet and activity 'substitute': parent intervention as in parent-only intervention and children and parents intervention focusing on increasing low-fat milk intake and decreasing TV watching

Healthy lifestyle group: delivered in 8 sessions, included recommendations on specific core food servings; practical skills for healthy eating, reduced sedentary behaviours and increased activity; and monitoring of lifestyle behaviours and roles and responsibilities around eating, managing appetite, self esteem and 
Collins $2011 \quad$ Parent-only diet: includes behavioural techniques for food choices
C1: parent-child (physical activity): includes physical activity goals and key skills

C2: parent-child (physical activity and diet): incorporates both the physical activity and diet-only components of the other interventions

Boutelle 2011 Parent-only behavioural change: included self monitoring of targeted behaviours, positive reinforcement, stimulus control, pre-planning and modelling

Parent-child behavioural: included self monitoring of targeted behaviours, positive reinforcement, stimulus control, pre-planning and modelling for parents and material taught in the child groups was similar but presented in an age-appropriate manner

West 2010

Parent-only behavioural change, 'Group Lifestyle Triple P': included positive-parenting strategies, physical activity strategies and nutrition strategies

Resnick 2009 Educational material plus personal encounters: included choices of topics such as biological, social and environmental influences on childhood overweight; nutrition advice, physical activity guidelines

Estabrooks 2009 Parent group and IVR counselling: 2 group sessions addressing behavioural health skills and knowledge of weight, nutrition and physical activity. Based on socialecological theory. Utilised the workbook in the control group and followed by 10 IVR sessions including prompts for physical activity, nutrition, behavioural components

Group sessions: 2 group sessions as per group + IVR intervention; utilised the workbook

Waiting list control: included a physical activity and nutritional advice components

Educational material: included components on increased physical activity and nutritional components Control: workbook group: 61-page workbook to
mote physical activity, healthy habits, nutrition

Munsch 2008 Mother-only CBT: included nutrition and eating behaviour, physical activity, social competences, body concept, relapse prevention; children attended a relaxation training session

Mother-child CBT: included the same components as the mother-only intervention; children received sessions on nutrition and eating behaviour, basic nutritional education, reinforcement and tokens, lessons in physical activity, social competencies, developing a positive body concept, relapse prevention

Janicke 2008 Parent-only: includes behavioural, nutritional and physical activity components

C1: parent-child: includes behavioural, nutritional and physical activity components

C2: waiting list control: no active treatment

Golley 2007

Parenting-skills training with intensive lifestyle education: behavioural change intervention including nutritional components; also 7 intensive lifestyle sup-
C1: parenting-skills training: behavioural change intervention including nutritional components 
port group sessions

C2: waiting list control: general healthy-lifestyle pamphlet

Golan 2006 Parent-only: included behavioural, nutritional and physical activity components
Parent-child: similar to the parent-only but including activities for the children

Aragona 1975

I1: parent-only response-cost plus reinforcement:

Waiting list control nutritional information, exercise instructions, weight and calorie information, behavioural techniques including reinforcement techniques

I2: parent-only - response-cost: as above without the reinforcement techniques

I: intervention; C: comparator; CBT: cognitive behavioural therapy; IVR: interactive voice response; NOURISH: Nourishing Our Understanding of Role modelling to Improve Support and Health; PCP: primary care providers; PHL: parent healthy lifestyle; TV: television

\section{Appendix 3. Baseline characteristics (I)}

\begin{tabular}{|c|c|c|c|c|c|c|c|c|}
\hline & $\begin{array}{l}\text { Interven- } \\
\text { tion(s) and } \\
\text { comparator } \\
\text { (s) }\end{array}$ & $\begin{array}{l}\text { Duration } \\
\text { of interven- } \\
\text { tion } \\
\text { (dura- } \\
\text { tion of fol- } \\
\text { low-up) }\end{array}$ & $\begin{array}{l}\text { Descrip- } \\
\text { tion of par- } \\
\text { ticipants }\end{array}$ & $\begin{array}{l}\text { Study } \\
\text { period } \\
\text { (year to } \\
\text { year) }\end{array}$ & Country & Setting & $\begin{array}{l}\text { Ethnic } \\
\text { groups } \\
(\%)\end{array}$ & $\begin{array}{l}\text { Socioeco- } \\
\text { nomic sta- } \\
\text { tus } \\
(\%)\end{array}$ \\
\hline \multirow[t]{2}{*}{$\begin{array}{l}\text { Resnicow } \\
2015\end{array}$} & $\begin{array}{l}\text { I1: parent- } \\
\text { only } \\
\text { PCP moti- } \\
\text { vational in- } \\
\text { terviewing }\end{array}$ & \multirow[t]{2}{*}{$\begin{array}{l}2 \text { years (fol- } \\
\text { low-up: } 2 \\
\text { years) }\end{array}$} & \multirow[t]{2}{*}{$\begin{array}{l}\text { Par- } \\
\text { ents of chil- } \\
\text { dren aged 2- } \\
8 \text { years with } \\
\text { BMI } \geq 85 \text { th } \\
\text { and } \leq 97 \text { th } \\
\text { percentile }\end{array}$} & \multirow[t]{2}{*}{-} & \multirow[t]{2}{*}{ USA } & \multirow[t]{2}{*}{ Primary care } & $\begin{array}{l}\text { White: } 53.6 \\
\text { Black: } 11.0 \\
\text { Hispanic: } \\
30.14 \\
\text { Asian: } 1.14 \\
\text { Other: } 3.83\end{array}$ & $\begin{array}{l}\text { Income } \\
<\text { USD 40, } \\
\text { 000: } 38.6 \% ; \\
\geq \text { USD 40, } \\
\text { 000: } 61.4 \% \\
\text { Education < } \\
\text { college: } 70 \\
1 \% \text {; } \geq \text { col- } \\
\text { lege: } 29.9 \%\end{array}$ \\
\hline & $\begin{array}{l}\text { I2: parent- } \\
\text { only } \\
\text { PCP + dieti- } \\
\text { cian motiva- } \\
\text { tional inter- } \\
\text { viewing }\end{array}$ & & & & & & $\begin{array}{l}\text { White: } 59.1 \\
\text { Black: } 6.09 \\
\text { Hispanic: } \\
20.9 \\
\text { Asian: } 8.7\end{array}$ & $\begin{array}{l}\text { Income } \\
<\text { USD 40, } \\
\text { 000: } 29.8 \% \\
\geq \text { USD 40, } \\
000 ; 70.2 \%\end{array}$ \\
\hline
\end{tabular}




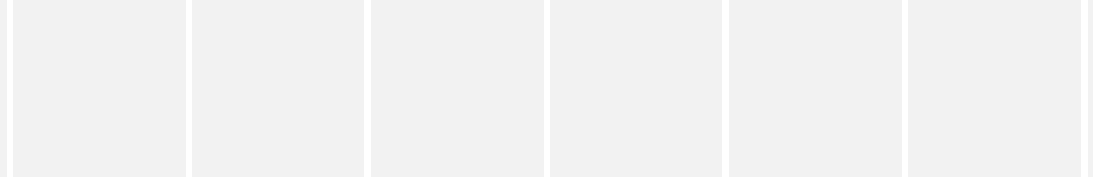

C: usual care
Other: 5.2 Education < college: 52 . $6 \%$; $\geq$ college: $47.4 \%$

White: 67.9 Income

Black: 2.6 < USD 40,

Hispanic: $\quad 000: 27.2 \%$;

13.3

Asian: 6.6

$\geq \operatorname{USD} 40$ 000: $72.8 \%$

Other: 9.7 Education < college: 61 . $8 \%$; $\geq$ college: $38.2 \%$

\begin{tabular}{|c|c|c|c|c|c|c|c|c|}
\hline \multirow[t]{2}{*}{$\begin{array}{l}\text { Mazzeo } \\
2014\end{array}$} & $\begin{array}{l}\text { I: parent } \\
\text { NOURISH }\end{array}$ & \multirow[t]{2}{*}{$\begin{array}{l}12 \text { weeks } \\
\text { (24 weeks) }\end{array}$} & \multirow{2}{*}{$\begin{array}{l}\text { Par- } \\
\text { ents of over- } \\
\text { weight chil- } \\
\text { dren aged 6- } \\
11 \text { years }\end{array}$} & \multirow[t]{2}{*}{ 2008-2009 } & \multirow[t]{2}{*}{ USA } & \multirow[t]{2}{*}{ Community } & \multirow[t]{2}{*}{ - } & \multirow[t]{2}{*}{ - } \\
\hline & $\begin{array}{l}\text { C: parent } \\
\text { control }\end{array}$ & & & & & & & \\
\hline \multirow[t]{2}{*}{$\begin{array}{l}\text { van } \\
\text { Grieken } \\
2013\end{array}$} & $\begin{array}{l}\text { I: parent- } \\
\text { only }\end{array}$ & \multirow[t]{2}{*}{$\begin{array}{l}\text { up to } 12 \\
\text { months (at } \\
24 \text { months) }\end{array}$} & \multirow[t]{2}{*}{$\begin{array}{l}\text { Par- } \\
\text { ents of over- } \\
\text { weight or } \\
\text { obese chil- } \\
\text { dren aged } 5 \\
\text { years }\end{array}$} & \multirow[t]{2}{*}{$\begin{array}{l}\text { September } \\
2007-\text { Octo- } \\
\text { ber } 2008\end{array}$} & \multirow[t]{2}{*}{$\begin{array}{l}\text { The Nether- } \\
\text { lands }\end{array}$} & \multirow[t]{2}{*}{$\begin{array}{l}\text { Commu- } \\
\text { nity (youth } \\
\text { health care } \\
\text { centres) }\end{array}$} & Dutch: 75.8 & $\begin{array}{l}\text { Mother's ed- } \\
\text { ucation level } \\
\text { low/mid: } \\
34 \text {. } \\
8 \text {; mid-high/ } \\
\text { high: } 65.2\end{array}$ \\
\hline & C: usual care & & & & & & Dutch: 80.6 & $\begin{array}{l}\text { Mother's ed- } \\
\text { ucation level } \\
\text { low/mid: } \\
31 . \\
\text { 5; mid-high/ } \\
\text { high: } 68.5\end{array}$ \\
\hline Small 2013 & $\begin{array}{l}\text { I: parent- } \\
\text { only }\end{array}$ & $\begin{array}{l}\text { 16-24 weeks } \\
(40-48 \\
\text { weeks) }\end{array}$ & $\begin{array}{l}\text { Par- } \\
\text { ents of over- } \\
\text { weight or } \\
\text { obese chil- } \\
\text { dren aged 4- } \\
8 \text { years }\end{array}$ & - & USA & $\begin{array}{l}\text { Primary care } \\
\text { office }\end{array}$ & $\begin{array}{l}\text { White: } 63 \\
\text { Black: } 9 \\
\text { Hispanic: } \\
24 \\
\text { Other: } 4\end{array}$ & $\begin{array}{l}\text { Mothers' ed- } \\
\text { ucation: } \\
\text { High school } \\
\text { degree or } \\
\text { less: } 24.3 \\
\text { At least } \\
\text { some } \\
\text { college: } 75.7 \\
\text { Mothers' } \\
\text { marital sta- } \\
\text { tus: } \\
\text { Married: } 75 . \\
7\end{array}$ \\
\hline
\end{tabular}




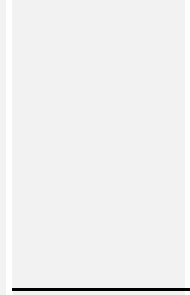

C: control
Single/di-

vorced/wid-

owed: 24.3

Family

structure:

2 parent: 97

1 parent: 3

White: 66

Hispanic:

25

Other: 7

Mothers' education:

High school degree or less: 29.7

At least some

college: 70.3

Mothers'

marital sta-

tus:

Married: 74.

1

Single/di-

vorced/wid-

owed: 25.9

Family

structure:

2 parent: 81 .

5

1 parent: 18 .

5

Father's edu-

cation

Under high

school

diploma: 4.3

High school

diploma: 41 .

4

Higher than high school diploma: 54.

3

Father's education

Under high school 


\begin{tabular}{|c|c|c|c|c|c|c|c|c|}
\hline & & & & & & & & $\begin{array}{l}\text { diploma: } 7 \\
\text { High school } \\
\text { diploma: } 38 . \\
4 \\
\text { Higher than } \\
\text { high school } \\
\text { diploma: } 54 . \\
7\end{array}$ \\
\hline \multirow[t]{2}{*}{$\begin{array}{l}\text { Moens } \\
2012\end{array}$} & $\begin{array}{l}\text { I: parent- } \\
\text { only }\end{array}$ & \multirow[t]{2}{*}{$\begin{array}{l}6 \text { months ( } 6 \\
\text { months) }\end{array}$} & \multirow[t]{2}{*}{$\begin{array}{l}\text { Par- } \\
\text { ents of over- } \\
\text { weight or } \\
\text { obese chil- } \\
\text { dren aged 6- } \\
12 \text { years }\end{array}$} & \multirow[t]{2}{*}{$2001-2006$} & \multirow[t]{2}{*}{ Belgium } & \multirow[t]{2}{*}{$\begin{array}{l}\text { University } \\
\text { research set- } \\
\text { ting }\end{array}$} & $\begin{array}{l}\text { 100\% Euro- } \\
\text { pean (Cau- } \\
\text { casian) }\end{array}$ & $\begin{array}{l}\text { Index } \\
\text { of social po- } \\
\text { sition, low } \\
26 \%, \text { mid- } \\
\text { dle } 52 \% \text {, } \\
\text { high } 22 \%\end{array}$ \\
\hline & $\begin{array}{l}\text { C: waiting } \\
\text { list control }\end{array}$ & & & & & & $\begin{array}{l}100 \% \text { Euro- } \\
\text { pean (Cau- } \\
\text { casian) }\end{array}$ & $\begin{array}{l}\text { Index } \\
\text { of social po- } \\
\text { sition, low } \\
0 \% \text {, middle } \\
75 \% \text {, high } \\
25 \%\end{array}$ \\
\hline \multirow[t]{3}{*}{$\begin{array}{l}\text { Raynor } \\
\text { 2012a }\end{array}$} & $\begin{array}{l}\text { I: parent- } \\
\text { only }\end{array}$ & \multirow[t]{3}{*}{$\begin{array}{l}24 \text { weeks } \\
\text { (12 months) }\end{array}$} & \multirow{3}{*}{$\begin{array}{l}\text { Parents of } \\
\text { overweight } \\
\text { or obese } \\
\text { children }\end{array}$} & \multirow{3}{*}{$\begin{array}{l}\text { November } \\
2005- \\
\text { September } \\
2007\end{array}$} & \multirow[t]{3}{*}{ USA } & \multirow[t]{3}{*}{$\begin{array}{l}\text { University } \\
\text { and primary } \\
\text { care }\end{array}$} & $\begin{array}{l}\text { White: } 90.9 \\
\text { Hispanic: } \\
21.2\end{array}$ & - \\
\hline & $\begin{array}{l}\mathrm{C} 1 \text { : parent - } \\
\text { diet decrease }\end{array}$ & & & & & & $\begin{array}{l}\text { White: } 80 \\
\text { Hispanic: } \\
20\end{array}$ & \\
\hline & $\begin{array}{l}\text { C2: parent - } \\
\text { diet increase }\end{array}$ & & & & & & $\begin{array}{l}\text { White: } 87 \\
\text { Hispanic: } \\
15.2\end{array}$ & \\
\hline \multirow[t]{3}{*}{$\begin{array}{l}\text { Raynor } \\
2012 b\end{array}$} & $\begin{array}{l}\text { I: parent- } \\
\text { only }\end{array}$ & \multirow[t]{3}{*}{$\begin{array}{l}24 \text { weeks } \\
(12 \text { months })\end{array}$} & \multirow{3}{*}{$\begin{array}{l}\text { Parents of } \\
\text { overweight } \\
\text { or obese } \\
\text { children }\end{array}$} & \multirow{3}{*}{$\begin{array}{l}\text { November } \\
2005- \\
\text { September } \\
2007\end{array}$} & \multirow[t]{3}{*}{ USA } & \multirow[t]{3}{*}{$\begin{array}{l}\text { University } \\
\text { and primary } \\
\text { care }\end{array}$} & $\begin{array}{l}\text { White: } 93.1 \\
\text { Hispanic: } \\
13.8\end{array}$ & \multirow[t]{3}{*}{ - } \\
\hline & $\begin{array}{l}\mathrm{C} 1 \text { : parent - } \\
\text { diet and ac- } \\
\text { tivity tradi- } \\
\text { tional }\end{array}$ & & & & & & $\begin{array}{l}\text { White: } 84.6 \\
\text { Hispanic: } \\
11.5\end{array}$ & \\
\hline & $\begin{array}{l}\text { C2: parent - } \\
\text { diet and ac- } \\
\text { tivity substi- } \\
\text { tute }\end{array}$ & & & & & & $\begin{array}{l}\text { White: } 92.3 \\
\text { Hispanic: } 7 . \\
7\end{array}$ & \\
\hline
\end{tabular}




\begin{tabular}{|c|c|c|c|c|c|c|c|c|}
\hline \multirow[t]{2}{*}{$\begin{array}{l}\text { Magarey } \\
2011\end{array}$} & $\begin{array}{l}\text { I: par- } \\
\text { ent healthy } \\
\text { lifestyle }\end{array}$ & \multirow[t]{2}{*}{$\begin{array}{l}24 \quad \text { weeks } \\
(104 \text { weeks })\end{array}$} & \multirow{2}{*}{$\begin{array}{l}\text { Par- } \\
\text { ents of chil- } \\
\text { dren classi- } \\
\text { fied as over- } \\
\text { weight aged } \\
5-9 \text { years }\end{array}$} & \multirow[t]{2}{*}{$\begin{array}{l}\text { May 2004- } \\
\text { estimated } \\
\text { May } 2006\end{array}$} & \multirow[t]{2}{*}{ Australia } & \multirow[t]{2}{*}{$\begin{array}{l}\text { Outpatient } \\
\text { clinic }\end{array}$} & \multirow[t]{2}{*}{ - } & \multirow{2}{*}{$\begin{array}{l}\text { Reported for } \\
\text { area of resi- } \\
\text { dence (Syd- } \\
\text { ney or Ade- } \\
\text { laide) only, } \\
\text { not by study } \\
\text { arm }\end{array}$} \\
\hline & $\begin{array}{l}\text { C: healthy } \\
\text { lifestyle }\end{array}$ & & & & & & & \\
\hline \multirow[t]{2}{*}{$\begin{array}{l}\text { Jansen } \\
2011\end{array}$} & $\begin{array}{l}\text { I: parent } \\
\text { CBT }\end{array}$ & \multirow[t]{2}{*}{$\begin{array}{l}12 \text { weeks } \\
\text { ( } 24 \text { weeks) }\end{array}$} & \multirow{2}{*}{$\begin{array}{l}\text { Par- } \\
\text { ents of over- } \\
\text { weight chil- } \\
\text { dren aged 7- } \\
13 \text { years }\end{array}$} & \multirow[t]{2}{*}{ - } & \multirow[t]{2}{*}{ Netherlands } & \multirow{2}{*}{$\begin{array}{l}\text { Community } \\
\text { health cen- } \\
\text { tre, univer- } \\
\text { sity site }\end{array}$} & \multirow[t]{2}{*}{ - } & \multirow[t]{2}{*}{ - } \\
\hline & $\begin{array}{l}\text { C: waiting } \\
\text { list control }\end{array}$ & & & & & & & \\
\hline \multirow[t]{3}{*}{$\begin{array}{l}\text { Collins } \\
2011\end{array}$} & $\begin{array}{l}\text { I: parent- } \\
\text { only diet }\end{array}$ & \multirow[t]{3}{*}{$\begin{array}{l}24 \quad \text { weeks } \\
\text { (104 weeks) }\end{array}$} & \multirow{3}{*}{$\begin{array}{l}\text { Par- } \\
\text { ents of over- } \\
\text { weight chil- } \\
\text { dren aged 5- } \\
9 \text { years }\end{array}$} & \multirow[t]{3}{*}{$\begin{array}{l}\text { January } \\
2005-2008\end{array}$} & \multirow[t]{3}{*}{ Australia } & \multirow[t]{3}{*}{$\begin{array}{l}\text { Outpatient } \\
\text { clinic }\end{array}$} & \multirow[t]{3}{*}{-} & \multirow[t]{3}{*}{ - } \\
\hline & $\begin{array}{l}\text { C1: parent- } \\
\text { child (physi- } \\
\text { cal activity) }\end{array}$ & & & & & & & \\
\hline & $\begin{array}{l}\text { C2: parent- } \\
\text { child (physi- } \\
\text { cal activity + } \\
\text { diet) }\end{array}$ & & & & & & & \\
\hline $\begin{array}{l}\text { Boutelle } \\
2011\end{array}$ & $\begin{array}{l}\text { I: parent- } \\
\text { only }\end{array}$ & $\begin{array}{l}20 \quad \text { weeks } \\
\text { (44 weeks) }\end{array}$ & $\begin{array}{l}\text { Parents and } \\
\text { their } \\
\text { overweight } \\
\text { or obese } \\
\text { (> 85th BMI } \\
\text { per- } \\
\text { centile) chil- } \\
\text { dren aged 8- } \\
12 \text { years }\end{array}$ & - & USA & University & - & $\begin{array}{l}\text { Total house- } \\
\text { hold } \\
\text { income: } \\
<\text { USD 20, } \\
000: 2.6 \% \\
\text { USD 20, } \\
001-40,000 \text { : } \\
7.9 \% \\
\text { USD 40, } \\
001-60,000: \\
18.4 \% \\
>\quad \$ 60,000: \\
63.2 \% \\
\text { Don't know: } \\
7.9 \% \\
\text { Education- } \\
\text { highest par- } \\
\text { ent: } \\
\text { Less than } \\
\text { high school } \\
0.0 \%\end{array}$ \\
\hline
\end{tabular}




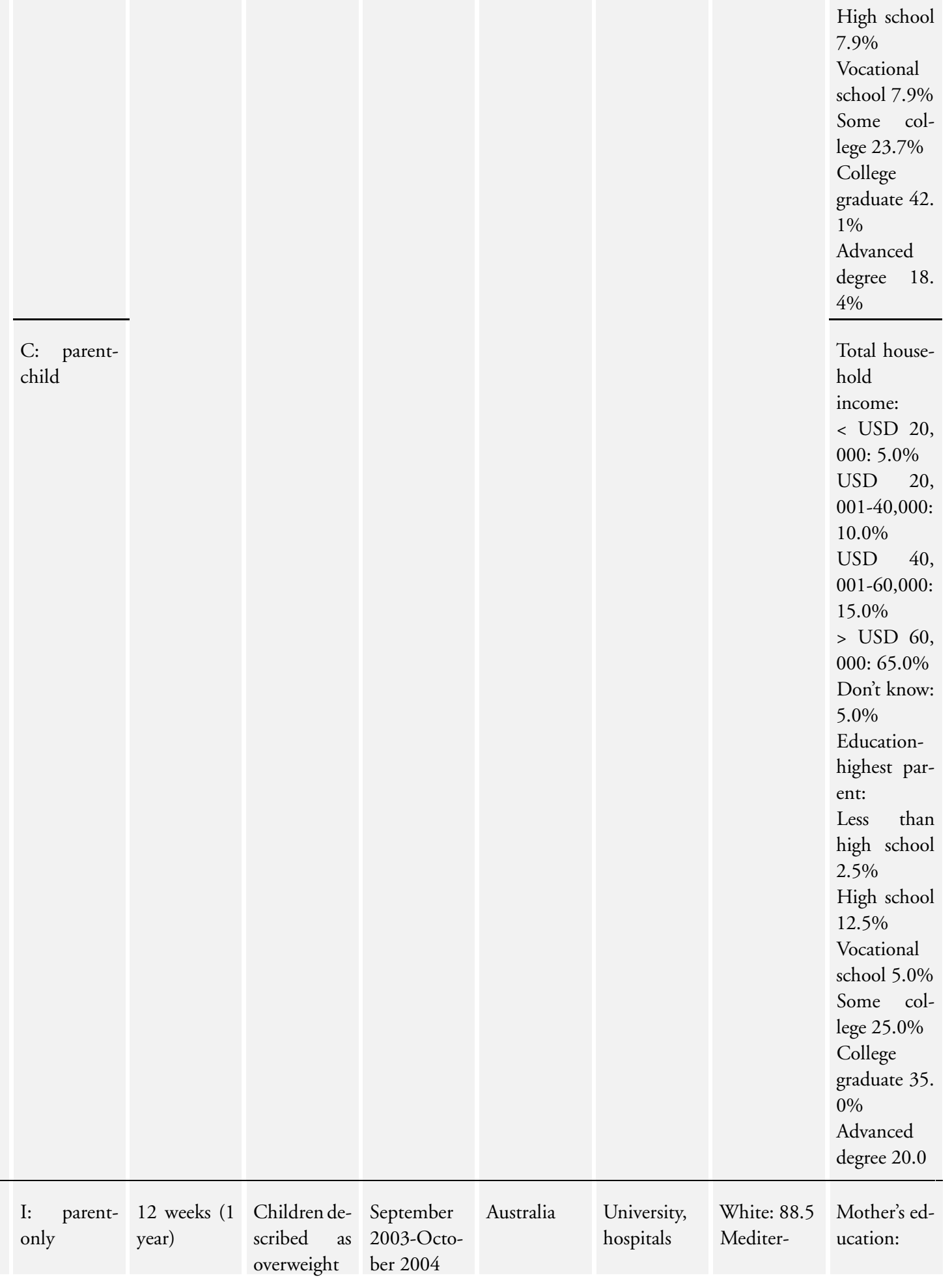




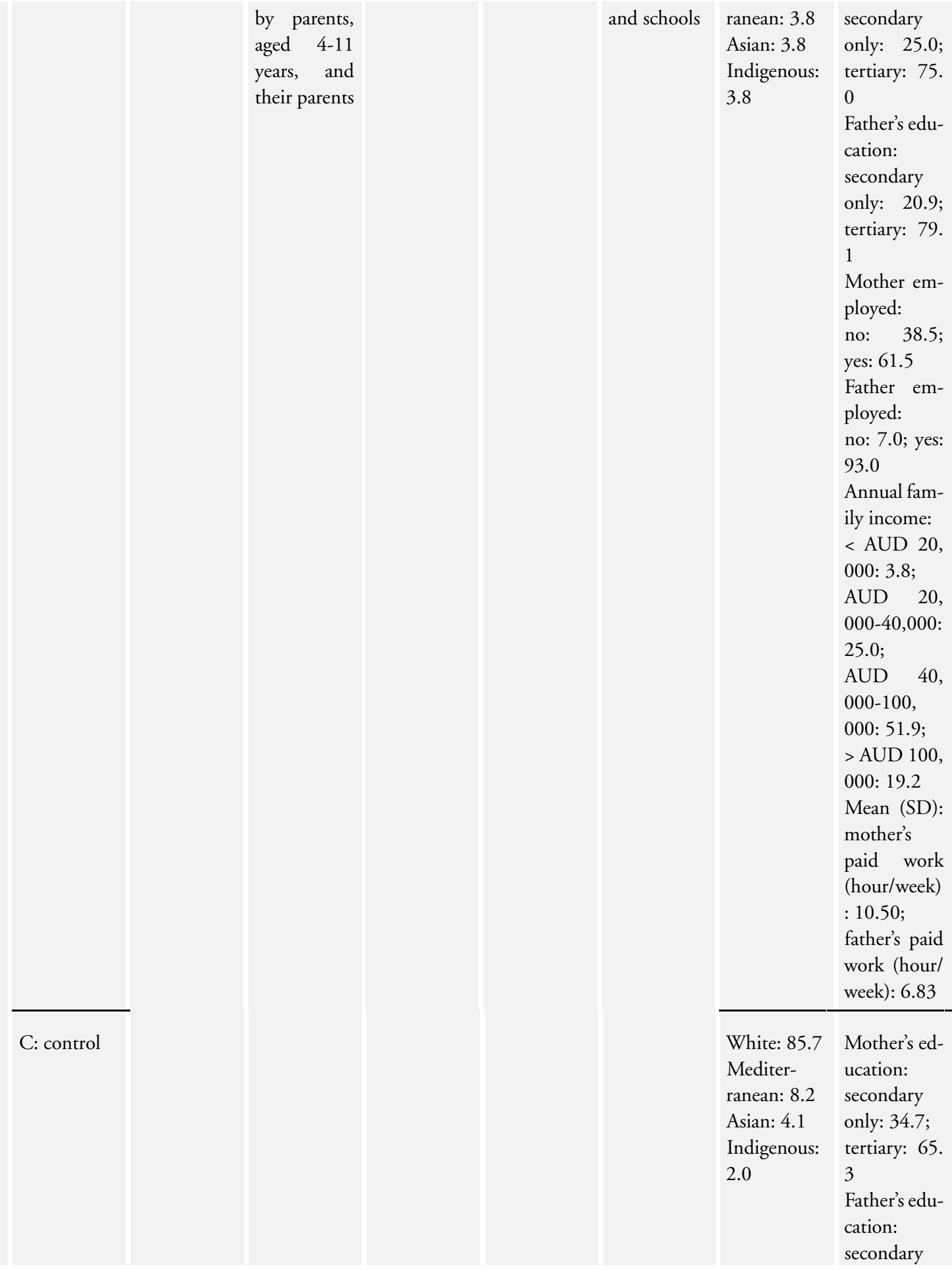


Mother em-

ployed:

no: 32.7 ;

yes: 67.3

Father employed:

no: 5.1; yes: 94.9

Annual family income: < AUD 20, 000: 0;

AUD 20, 000-40,000: 22.4;

AUD 40, 000-100, 000: 51.0; $>$ AUD 100, 000: 26.5 Mean (SD): mother's paid work (hour/week) : 12.33; father's paid work (hour/ week): 11 . 91

\begin{tabular}{|c|c|c|c|c|c|c|c|c|}
\hline \multirow[t]{2}{*}{$\begin{array}{l}\text { Resnick } \\
2009\end{array}$} & $\begin{array}{l}\text { I: edu- } \\
\text { cational ma- } \\
\text { terial } \quad+ \\
\text { personal en- } \\
\text { counters }\end{array}$ & \multirow[t]{2}{*}{$\begin{array}{l}18 \\
\text { weeks (un- } \\
\text { clear follow- } \\
\text { up, } 41 \text { week } \\
\text { mail out) }\end{array}$} & \multirow[t]{2}{*}{$\begin{array}{l}\text { Par- } \\
\text { ents of over- } \\
\text { weight chil- } \\
\text { dren aged 5- } \\
11 \text { years }\end{array}$} & \multirow[t]{2}{*}{$\begin{array}{l}\text { October } \\
\text { 2006- } \\
\text { April } 2007\end{array}$} & \multirow[t]{2}{*}{ USA } & \multirow[t]{2}{*}{ Community } & \multirow[t]{2}{*}{ - } & \multirow[t]{2}{*}{ - } \\
\hline & $\begin{array}{l}\mathrm{C}: \text { edu- } \\
\text { cational ma- } \\
\text { terial }\end{array}$ & & & & & & & \\
\hline $\begin{array}{l}\text { Estabrooks } \\
2009\end{array}$ & $\begin{array}{l}\mathrm{I}: \quad \text { parent } \\
\text { group }+ \text { IVR }\end{array}$ & $\begin{array}{l}12-24 \text { weeks } \\
\text { (52 weeks) }\end{array}$ & $\begin{array}{l}\text { Par- } \\
\text { ents of chil- } \\
\text { dren aged 8- } \\
12 \\
\text { years with a } \\
\text { BMI > 85th }\end{array}$ & $\begin{array}{l}\text { May 2004- } \\
\text { December } \\
2007\end{array}$ & USA & $\begin{array}{l}\text { Outpatient } \\
\text { clinic }\end{array}$ & $\begin{array}{l}\text { White: } 60 \\
\text { Hispanic: } \\
30\end{array}$ & - \\
\hline
\end{tabular}




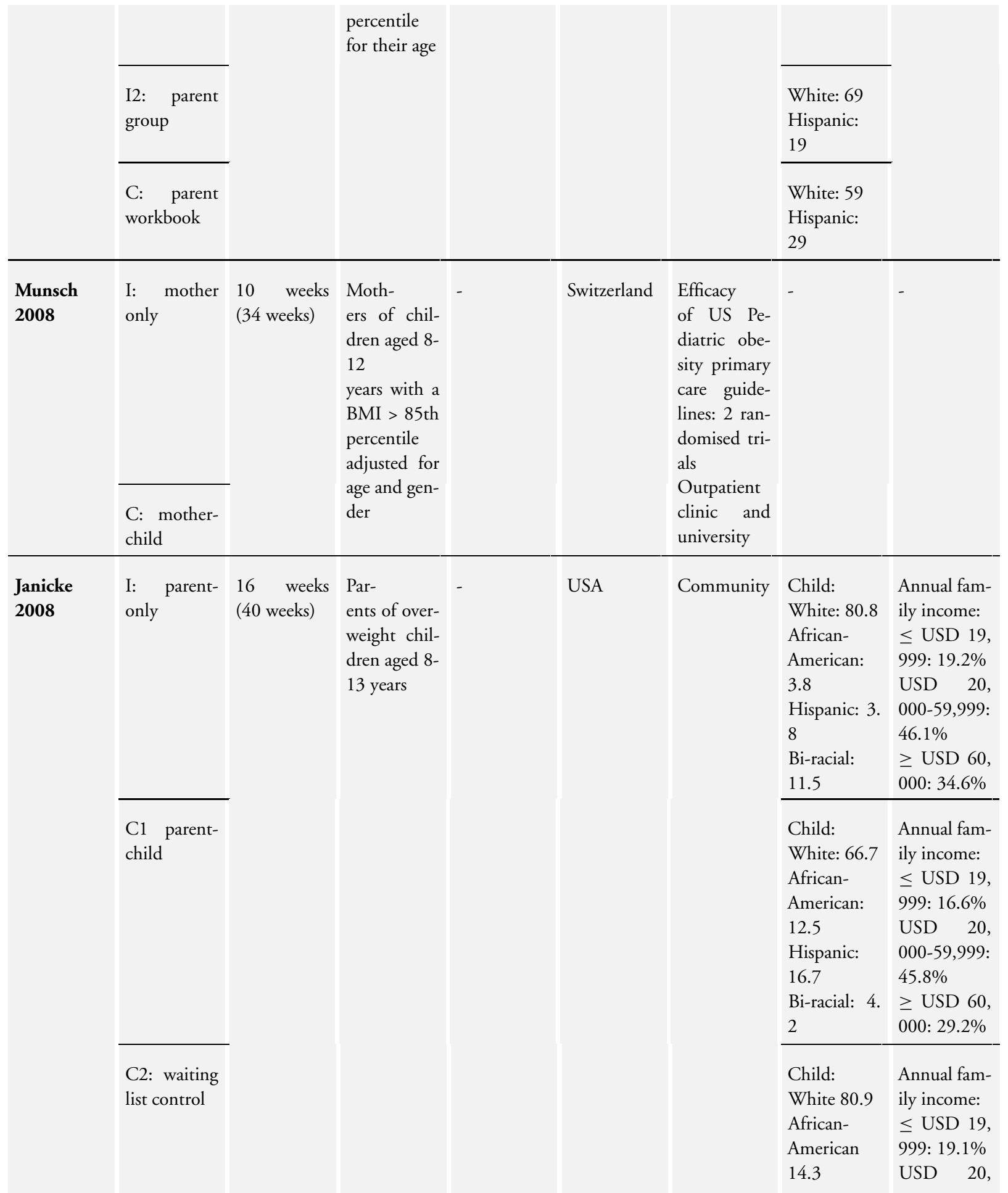




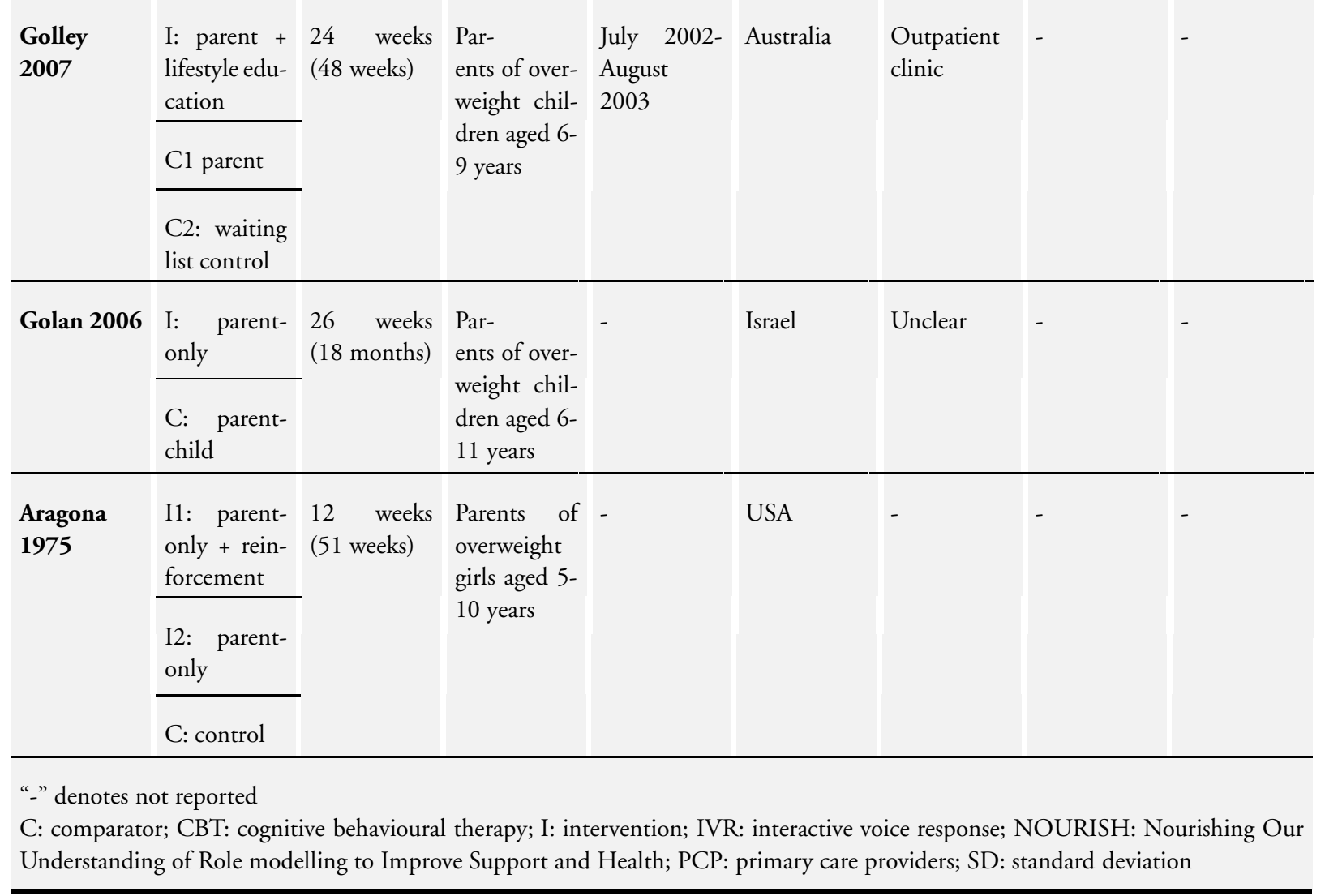

\section{Appendix 4. Baseline characteristics (II)}

\begin{tabular}{|c|c|c|c|c|c|c|c|}
\hline & $\begin{array}{l}\text { Intervention } \\
\text { (s) and } \\
\text { comparator } \\
\text { (s) }\end{array}$ & $\begin{array}{l}\text { Sex } \\
{[\text { female \% }]^{a}}\end{array}$ & $\begin{array}{l}\text { Age } \\
\text { [mean (SD)] }\end{array}$ & $\begin{array}{l}\text { BMI } \\
\text { measures } \\
{\left[\text { mean } \mathrm{kg} / \mathrm{m}^{2}\right.} \\
(\mathrm{SD})]\end{array}$ & $\begin{array}{l}\text { Body weight } \\
{[\text { mean kg }} \\
(\mathrm{SD})]\end{array}$ & $\begin{array}{l}\text { Parental } \\
\text { weight } \\
\text { [mean } \\
\text { BMI (SD) or } \\
\text { as stated] }\end{array}$ & $\begin{array}{l}\text { Comedica- } \\
\text { tions/coint- } \\
\text { erventions/ } \\
\text { comorbidi- } \\
\text { ties }\end{array}$ \\
\hline $\begin{array}{l}\text { Resnicow } \\
2015\end{array}$ & $\begin{array}{l}\text { I1: parent- } \\
\text { only PCP mo- } \\
\text { tivational in- } \\
\text { terviewing }\end{array}$ & 57 & $5.1(1.9)$ & $\begin{array}{l}\text { Percentile: } 92 . \\
2(3.3)\end{array}$ & - & $\begin{array}{l}\text { BMI: } 30.1(7 . \\
4)\end{array}$ & - \\
\hline
\end{tabular}




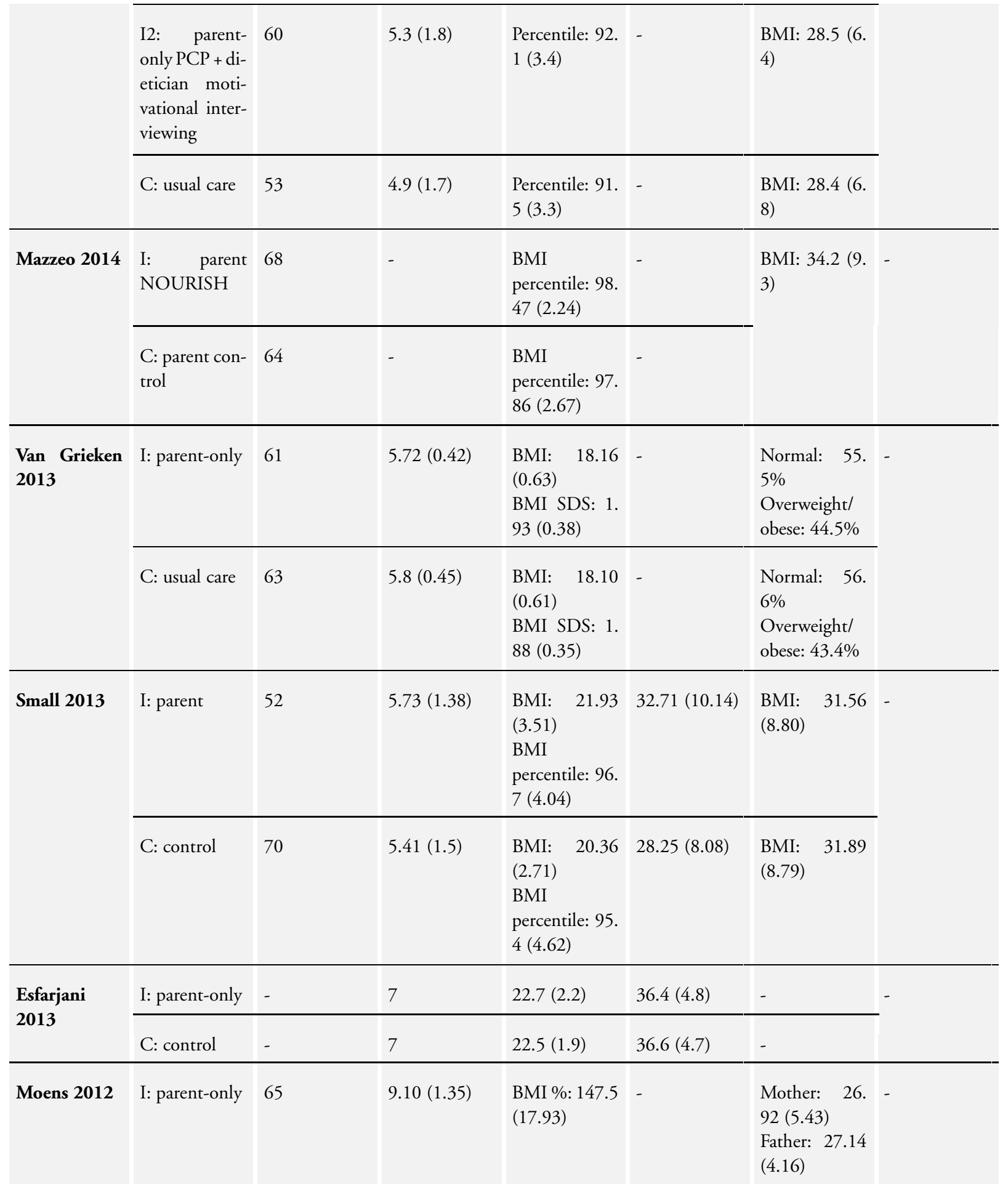




\begin{tabular}{|c|c|c|c|c|c|c|c|}
\hline & $\begin{array}{l}\text { C: waiting list } \\
\text { control }\end{array}$ & 58 & $9.26(1.45)$ & $\begin{array}{l}\text { BMI \%: } 140 . \\
45(10.15)\end{array}$ & - & $\begin{array}{l}\text { Mother: } 24 . \\
75(3.02) \\
\text { Father: } 28.16 \\
(3.15)\end{array}$ & \\
\hline \multirow[t]{3}{*}{$\begin{array}{l}\text { Raynor } \\
\text { 2012a }\end{array}$} & I: parent-only & 61 & $6.8(1.8)$ & $\begin{array}{l}\text { BMI: } 34.6 \pm 9 . \\
7\end{array}$ & - & - & - \\
\hline & $\begin{array}{l}\text { C1: parent - } \\
\text { diet decrease }\end{array}$ & 63 & $7.2(1.6)$ & $\begin{array}{l}\text { BMI: } 33.4 \pm 8 \\
3\end{array}$ & - & - & \\
\hline & $\begin{array}{l}\text { C2: parent - } \\
\text { diet increase }\end{array}$ & 61 & $7.6(1.6)$ & $\begin{array}{l}\text { BMI: } 32.2 \pm 7 \\
2\end{array}$ & - & - & \\
\hline \multirow[t]{3}{*}{$\begin{array}{l}\text { Raynor } \\
2012 b\end{array}$} & I: parent-only & 59 & 6.7 (1.6) & $\begin{array}{l}\text { BMI: } 33.2 \pm 9 . \\
1\end{array}$ & - & - & - \\
\hline & $\begin{array}{l}\mathrm{C} 1 \text { : parent - } \\
\text { diet + activity } \\
\text { traditional }\end{array}$ & 65 & $7.2(1.5)$ & $\begin{array}{l}\text { BMI: } 30.5 \pm 7 . \\
2\end{array}$ & - & - & \\
\hline & $\begin{array}{l}\text { C2: parent - } \\
\text { diet + activity } \\
\text { substitute }\end{array}$ & 58 & $7.4(1.3)$ & $\begin{array}{l}\text { BMI: } 33.6 \pm 8 \\
5\end{array}$ & - & - & \\
\hline \multirow[t]{2}{*}{$\begin{array}{l}\text { Margarey } \\
2011\end{array}$} & $\begin{array}{l}\text { I: } \\
\text { parent healthy } \\
\text { lifestyle }\end{array}$ & 56 & All: $8.2(1.2)$ & $\begin{array}{l}\text { BMI (all): } 24 . \\
1(3.22) \\
\text { BMI z score: } \\
2.77(0.58)\end{array}$ & $\begin{array}{ll}\text { All: } & 44.4 \quad \text { (9. } \\
82) & \end{array}$ & - & - \\
\hline & $\begin{array}{l}\text { C: healthy } \\
\text { lifestyle }\end{array}$ & All: 56 & All: $8.2(1.2)$ & $\begin{array}{l}\text { BMI (all): } 24 . \\
1(3.22) \\
\text { BMI z score } 2 . \\
68(0.65)\end{array}$ & $\begin{array}{l}\text { All: } 44.4 \quad \text { (9. } \\
82)\end{array}$ & - & \\
\hline \multirow[t]{2}{*}{ Jansen 2011} & I: parent CBT & - & - & $\begin{array}{l}\text { BMI } \\
\text { percentile: } 96 . \\
8(2.93)\end{array}$ & - & $\begin{array}{ll}\text { BMI: } & 28.30 \\
(4.53) & \end{array}$ & - \\
\hline & $\begin{array}{l}\text { C: waiting list } \\
\text { control }\end{array}$ & - & - & $\begin{array}{l}\text { BMI } \\
\text { percentile: } 95 . \\
9(3.38)\end{array}$ & - & $\begin{array}{ll}\text { BMI: } & 29.35 \\
(6.33) & \end{array}$ & \\
\hline Collins 2011 & $\begin{array}{l}\text { I: parent-only } \\
\text { diet }\end{array}$ & 62 & $8.2(1.2)$ & $\begin{array}{l}\text { BMI: } 24.6(3 . \\
0) \\
\text { BMI z score: } \\
2.8(0.6)\end{array}$ & $46.3(8.6)$ & - & - \\
\hline
\end{tabular}




\begin{tabular}{|c|c|c|c|c|c|c|c|}
\hline & $\begin{array}{l}\mathrm{C} 1 \text { : parent- } \\
\text { child (physical } \\
\text { activity) }\end{array}$ & 60 & $8.3(1.0)$ & $\begin{array}{l}\text { BMI: } 25.2 \text { (4. } \\
\text { 1) } \\
\text { BMI z score: } \\
2.8(0.7)\end{array}$ & $48(10.8)$ & - & - \\
\hline & $\begin{array}{l}\mathrm{C} 2: \text { parent- } \\
\text { child (physical } \\
\text { activity + diet) }\end{array}$ & 55 & $8.1(1.2)$ & $\begin{array}{l}\text { BMI: } 24.4(3 . \\
7) \\
\text { BMI z score: } \\
2.8(0.7)\end{array}$ & $45.5(12.2)$ & - & - \\
\hline \multirow[t]{2}{*}{$\begin{array}{l}\text { Boutelle } \\
2011\end{array}$} & I: parent-only & 50 & $10.81(1.31)$ & $\begin{array}{l}\text { Child BMI } \\
\text { percentile: } 98 . \\
37(1.85) \\
\text { Child BMI z } \\
\text { score: } 2.29(0 . \\
38) \\
\text { Child BMI: } \\
30.48(6.08)\end{array}$ & - & $\begin{array}{l}\text { Parent BMI: } \\
32.47(8.25) \\
\mathrm{n}=39\end{array}$ & - \\
\hline & $\begin{array}{l}\text { C: parent- } \\
\text { child }\end{array}$ & 70 & $10.08(1.15)$ & $\begin{array}{l}\text { Child BMI } \\
\text { percentile: } 98 . \\
34(1.37) \\
\text { Child BMI z } \\
\text { score: } 2.25(0 . \\
34) \\
\text { Child BMI: } \\
28.26(4.64)\end{array}$ & - & $\begin{array}{l}\text { Parent BMI: } \\
31.47(7.46) \\
\mathrm{n}=40\end{array}$ & \\
\hline \multirow[t]{2}{*}{ West 2010} & I: parent-only & 69 & 8.58 (1.69) & $\begin{array}{l}\text { BMI z score: } \\
2.15(0.43)\end{array}$ & - & $\begin{array}{l}\text { Parent BMI } \\
\text { range (n, \%) } \\
\text { Healthy } \\
\text { weight: } 20 \\
(38.5) \\
\text { Overweight: } \\
11(21.2) \\
\text { Obese: } 21 \text { (40. } \\
4)\end{array}$ & - \\
\hline & C: control & 65 & $8.5(1.65)$ & $\begin{array}{l}\text { BMI z score: } \\
2.11(0.46)\end{array}$ & - & $\begin{array}{l}\text { Parent BMI } \\
\text { range (n, \%) } \\
\text { Healthy } \\
\text { weight: } 18 \\
(36.7) \\
\text { Overweight: } \\
12(24.5) \\
\text { Obese: } 19(38 . \\
8)\end{array}$ & \\
\hline
\end{tabular}




\begin{tabular}{|c|c|c|c|c|c|c|c|}
\hline \multirow[t]{2}{*}{ Resnick 2009} & $\begin{array}{l}\text { I: educational } \\
\text { material + per- } \\
\text { sonal encoun- } \\
\text { ters }\end{array}$ & - & - & $\begin{array}{l}\text { BMI } \\
\text { percentile: } 94 . \\
1(4.3)\end{array}$ & - & BMI: 25.6 & - \\
\hline & $\begin{array}{l}\text { C: educational } \\
\text { material }\end{array}$ & - & - & $\begin{array}{l}\text { BMI } \\
\text { percentile: } 94 . \\
1(4.4)\end{array}$ & - & BMI: 26.2 & \\
\hline \multirow[t]{3}{*}{$\begin{array}{l}\text { Estabrooks } \\
2009\end{array}$} & $\begin{array}{l}\mathrm{I}: \quad \text { parent } \\
\text { group }+ \text { IVR }\end{array}$ & 41 & 10.7 & $\begin{array}{l}\text { BMI: } 27.1 \\
\text { BMI z score: } \\
2.04\end{array}$ & - & - & - \\
\hline & $\begin{array}{l}\text { I2: parent } \\
\text { group }\end{array}$ & 42 & 10.6 & $\begin{array}{l}\text { BMI: } 27.4 \\
\text { BMI z score: } \\
2.07\end{array}$ & - & - & \\
\hline & $\begin{array}{l}\text { C: parent } \\
\text { workbook }\end{array}$ & 61 & 11 & $\begin{array}{l}\text { BMI: } 27.1 \\
\text { BMI z score: } \\
2.00\end{array}$ & - & - & \\
\hline \multirow[t]{2}{*}{ Munsch 2008} & $\begin{array}{l}\text { I: mother- } \\
\text { only }\end{array}$ & 63 & $10.6(1.5)$ & $\begin{array}{l}\text { BMI: } 28.0 \\
4), \mathrm{n}=21 \\
\text { BMI SDS: } 2 . \\
61\end{array}$ & - & $\begin{array}{l}\text { BMI: } 26.9(3 . \\
9)\end{array}$ & - \\
\hline & $\begin{array}{l}\text { C: mother- } \\
\text { child }\end{array}$ & 59 & $10.3(1.4)$ & $\begin{array}{l}\text { BMI: } 26.5 \text { (3. } \\
3) \\
\text { BMI SDS: } 2 . \\
40\end{array}$ & - & $\begin{array}{l}\text { BMI: } 29.6(7 . \\
5)\end{array}$ & \\
\hline \multirow[t]{3}{*}{ Janicke 2008} & I: parent-only & 46 & $11.5(1.3)$ & $\begin{array}{l}\text { BMI z score: } \\
2.16(0.35)\end{array}$ & - & $\begin{array}{l}\text { BMI: } \quad 35.47 \\
(8.2)\end{array}$ & - \\
\hline & $\begin{array}{l}\text { C1 parent- } \\
\text { child }\end{array}$ & 63 & 11.03 (1.6) & $\begin{array}{l}\text { BMI z score: } \\
2.13(0.43)\end{array}$ & - & $\begin{array}{l}\text { BMI: } \quad 32.86 \\
(6.8)\end{array}$ & \\
\hline & $\begin{array}{l}\text { C2: waiting } \\
\text { list control }\end{array}$ & 76 & $11.02(1.81)$ & $\begin{array}{l}\text { BMI z score: } \\
2.02(0.41)\end{array}$ & - & $\begin{array}{l}\text { BMI: } \quad 35.66 \\
(9.3)\end{array}$ & \\
\hline \multirow[t]{3}{*}{ Golley 2007} & $\begin{array}{l}\text { I: parent } \\
+ \text { lifestyle edu- } \\
\text { cation }\end{array}$ & 63 & - & $\begin{array}{l}\text { BMI z score: } \\
2.74(0.58)\end{array}$ & - & - & - \\
\hline & $\mathrm{C} 1$ parent & 65 & - & $\begin{array}{l}\text { BMI z score: } \\
2.76(0.58)\end{array}$ & - & - & \\
\hline & $\begin{array}{l}\text { C2: waiting } \\
\text { list control }\end{array}$ & 64 & - & $\begin{array}{l}\text { BMI z score: } \\
2.75(0.39)\end{array}$ & - & - & \\
\hline
\end{tabular}




\begin{tabular}{|c|c|c|c|c|c|c|c|}
\hline \multirow[t]{2}{*}{ Golan 2006} & I: parent-only & 59 & 8.75 (1.9) & $24.2(3.0)$ & $47.1(12.4)$ & $\begin{array}{l}\text { Weight: } \mathrm{kg} \\
\text { Mothers: } 72.7 \\
(11.1) \\
\text { Fathers: } 100.9 \\
(24.7)\end{array}$ & \multirow[t]{2}{*}{ - } \\
\hline & $\begin{array}{l}\text { C: parent- } \\
\text { child }\end{array}$ & 50 & $8.7(2.0)$ & $24.3(3.6)$ & 45.5 (15.9) & $\begin{array}{l}\text { Weight: kg } \\
\text { Mothers: } 79.1 \\
\text { (15.5) } \\
\text { Fathers: } 102.3 \\
\text { (19.1) }\end{array}$ & \\
\hline \multirow[t]{3}{*}{$\begin{array}{l}\text { Aragona } \\
1975\end{array}$} & $\begin{array}{l}\text { I1: par- } \\
\text { ent-only + re- } \\
\text { inforcement }\end{array}$ & 100 & 9.4 & $\begin{array}{l}\mathrm{BMI}^{b}: 21.72 \\
(2.03)\end{array}$ & $47.89(7.76)$ & - & \multirow[t]{3}{*}{ - } \\
\hline & $\begin{array}{l}\text { I2: parent- } \\
\text { only }\end{array}$ & 100 & 10 & $\begin{array}{l}\mathrm{BMI}^{b}: 22.76 \\
(1.58)\end{array}$ & $47.42(5.70)$ & - & \\
\hline & C: control & 100 & 8.3 & $\begin{array}{l}\mathrm{BMI}^{b}: 23.06 \\
(3.04)\end{array}$ & $45.04(14.51)$ & - & \\
\hline
\end{tabular}

$\because$ denotes not reported

${ }^{a}$ Sex of the overweight child

${ }^{b}$ Calculated by review authors

BMI: body mass index; BMI SDS: standard deviation of BMI: C: comparator; CBT: cognitive behavioural therapy; I: intervention; IVR: interactive voice response; n: number of participants; NOURISH: Nourishing Our Understanding of Role modelling to Improve Support and Health; PCP: primary care providers; SD: standard deviation; TV: television

\section{Appendix 5. Matrix of study endpoints (publications and trial documents)}

\begin{tabular}{|c|c|c|c|c|}
\hline & $\begin{array}{l}\text { Endpoints quoted in } \\
\text { trial document(s) } \\
\text { (ClinicalTri- } \\
\text { als.gov, FDA/EMA doc- } \\
\text { ument, man- } \\
\text { ufacturer's website, pub- } \\
\text { lished design paper) }^{a}\end{array}$ & $\begin{array}{l}\text { Study results/ } \\
\text { publications available } \\
\text { in trials register }\end{array}$ & $\begin{array}{l}\text { Endpoints quoted in } \\
\text { publication }(\mathbf{s})^{b, c}\end{array}$ & $\begin{array}{l}\text { Endpoints quoted in ab- } \\
\text { stract of publication(s) } \\
b, c\end{array}$ \\
\hline Resnicow 2015 & $\begin{array}{l}\text { Source: NCT } 01335308 \\
\text { Primary outcome mea- } \\
\text { sure(s): BMI percentile }\end{array}$ & $\begin{array}{l}\text { No } \\
\text { (last verified: March } \\
\text { 2011) } \\
\text { History of changes: } 0 \\
\text { documented changes }\end{array}$ & $\begin{array}{l}\text { Primary outcome mea- } \\
\text { sure(s): BMI percentile }\end{array}$ & $\begin{array}{l}\text { Primary outcome mea- } \\
\text { sure(s): BMI percentile }\end{array}$ \\
\hline
\end{tabular}




Secondary out-
come measure(s): fruit +
vegetable consump-
tion, sweetened beverage
consumption, change in
physical activity

Other outcome measure

(s): -

\begin{tabular}{|l|l|}
\hline Mazzeo 2014 & $\begin{array}{l}\text { Source: NCT00628030 } \\
\text { Primary outcome mea- } \\
\text { sure(s): } \\
\bullet \text { child BMI }\end{array}$ \\
\hline $\begin{array}{l}\text { Secondary outcome } \\
\text { measure(s): } \\
\bullet \text { child feeding } \\
\bullet \text { child quality of life } \\
\bullet \text { parental BMI } \\
\bullet \text { parental dietary } \\
\text { intake }\end{array}$ \\
\hline Other outcome measure
\end{tabular}

(s): -

van Grieken 2013 Source:

ISRCTN04965410,

NTR921

Primary outcome measure(s): BMI, waist circumference

(last verified: July 2015)

History of changes: 7

$\begin{array}{ll}\begin{array}{l}\text { Secondary outcome } \\ \text { measure(s): - }\end{array} & \begin{array}{l}\text { Secondary outcome } \\ \text { measure(s): - }\end{array}\end{array}$

\section{Yes}

documented changes

$\begin{array}{ll}\text { (s): - } & (s):-\end{array}$

Primary outcome mea- Primary outcome measure(s): BMI sure(s): child BMI

Other outcome measure Other outcome measure (s): -

\begin{tabular}{|c|c|}
\hline $\begin{array}{l}\text { Secondary outcome } \\
\text { measure(s): Three Fac- } \\
\text { tor Eating Questionnaire; } \\
\text { Child Feeding Question- } \\
\text { naire; dietary intake } \\
\text { (Block Food Screener); } \\
\text { Pediatric Health-Related } \\
\text { Quality of Life; Pubertal } \\
\text { status (self report) }\end{array}$ & $\begin{array}{l}\text { Secondary outcome } \\
\text { measure (s): parents satis- } \\
\text { faction, parent behaviour } \\
\text { change }\end{array}$ \\
\hline
\end{tabular}

Other outcome measure Other outcome measure (s): (s): -

Primary outcome mea- Primary outcome measure(s): BMI, waist cir- sure(s): BMI cumference

last verified: July 2015)

History of changes: no

documented changes

Secondary out-
come measure(s): levels
of the 4 target overweight-
reducing and overweight-
inducing behaviours, i.
e. being physically ac-
tive, eating breakfast daily,
drinking sweet beverages
and watching TV or play-
ing on a computer; health-
related quality of life;
attitudes of parents re-
garding the 4 target be-
haviours; absence or pres-


ence of indicators of negative adverse effects, i. e. worry, stigmatisation, lowered self esteem and development of relative underweight

Other outcome measure

(s): -

\begin{tabular}{|c|c|c|}
\hline \multirow[t]{3}{*}{ Small 2013} & \multirow[t]{3}{*}{ Source: N/T } & $\begin{array}{l}\text { Primary outcome mea- } \\
\text { sure(s): - }\end{array}$ \\
\hline & & $\begin{array}{l}\text { Secondary outcome } \\
\text { measure(s): - }\end{array}$ \\
\hline & & $\begin{array}{l}\text { Outcomes not specified } \\
\text { as primary or secondary: } \\
\text { BMI percentile; waist cir- } \\
\text { cumference; waist for } \\
\text { height ratio }\end{array}$ \\
\hline \multirow[t]{3}{*}{ Esfarjani 2013} & \multirow[t]{3}{*}{ Source: N/T } & $\begin{array}{l}\text { Primary outcome mea- } \\
\text { sure(s): - }\end{array}$ \\
\hline & & $\begin{array}{l}\text { Secondary outcome } \\
\text { measure(s): - }\end{array}$ \\
\hline & & $\begin{array}{l}\text { Outcomes not reported } \\
\text { as primary or secondary: } \\
\text { height, } \\
\text { weight, BMI, waist cir- } \\
\text { cumference, hip circum- } \\
\text { ference, fasting blood glu- } \\
\text { cose (data not extracted) } \\
\text {, triglyceride (data not } \\
\text { extracted), total choles- } \\
\text { terol (data not extracted), } \\
\text { HDL-cholesterol } \\
\text { (data not extracted, LDL } \\
\text { cholesterol (data not ex- } \\
\text { tracted), food consump- } \\
\text { tion (data not extracted), } \\
\text { watching TV (data not ex- } \\
\text { tracted), playing on com- } \\
\text { puter (data not extracted), } \\
\text { walking time (data not ex- } \\
\text { tracted) }\end{array}$ \\
\hline
\end{tabular}

Other outcome measure Other outcome measure (s): -

Primary outcome measure(s): -

Secondary outcome measure(s): -

Other outcome measure(s): waist circumference, waist-by-height ratio, BMI and BMI percentile

Primary outcome measure(s): -

Secondary outcome
measure(s): -

Other outcome measure (s): weight, waist and hip circumference, cholesterol, serum triglycerides, food group consumption, TV and computer time, walking time 


\begin{tabular}{|c|c|c|c|}
\hline \multirow[t]{3}{*}{ Moens 2012} & \multirow[t]{3}{*}{ Source: N/T } & $\begin{array}{l}\text { Primary outcome mea- } \\
\text { sure(s): see below }\end{array}$ & $\begin{array}{l}\text { Primary outcome mea- } \\
\text { sure(s): - }\end{array}$ \\
\hline & & $\begin{array}{l}\text { Secondary outcome } \\
\text { measure(s): see below }\end{array}$ & $\begin{array}{l}\text { Secondary outcome } \\
\text { measure }(s):-\end{array}$ \\
\hline & & $\begin{array}{l}\text { Outcomes not reported } \\
\text { as primary or secondary: } \\
\text { height, weight, Dutch eat- } \\
\text { ing behaviour question- } \\
\text { naire (child and parent } \\
\text { versions); Ghent Parental } \\
\text { Behaviour Scale; health } \\
\text { principles questionnaire, } \\
\text { Hollingshead Index of So- } \\
\text { cial Position }\end{array}$ & $\begin{array}{l}\text { Other outcome measure } \\
\text { (s): BMI, parental re- } \\
\text { port of child's eating be- } \\
\text { haviour, familial health } \\
\text { principles }\end{array}$ \\
\hline
\end{tabular}

Raynor 2012b

Raynor 2012a
Source: NCT00259324

Primary outcome mea-

sure(s): BMI z score

Secondary outcome

measure(s): eating and ac-

tivity behaviours

Other outcome measure

(s):
No (last verified: July

2015)

History of changes: 6 documented changes 6 sure(s): -

Primary outcome measure(s): -
Source: NCT00200265

Primary outcome measure(s): BMI z score

Secondary outcome measure(s): eating and activity behaviours

Other outcome measure (s): -
No (last verified: July 2015) History of changes: 6 documented changes

(1)
Outcomes not specified as primary or secondary: weight, height, BMI and BMI z score; dietary intake (food diaries); leisure time activity (Previous Day Physical Activity Recall)
Other outcome measure (s): BMI z score, energy intake

Secondary measure(s): -

Primary outcome measure(s): -

Primary outcome measure(s): -

$\begin{array}{ll}\begin{array}{l}\text { Secondary outcome } \\ \text { measure }(s):-\end{array} & \begin{array}{l}\text { Secondary outcome } \\ \text { measure }(s):-\end{array}\end{array}$

Outcomes not specified Other outcome measure as primary or secondary: (s): BMI z score, energy weight, height, BMI and intake BMI z score; dietary intake (food diaries); leisure time activity (Previous Day Physical Activity Re- 
call)

\begin{tabular}{|c|c|c|c|c|}
\hline \multirow[t]{3}{*}{ Magarey 2011} & $\begin{array}{l}\text { Source: } \\
\text { ACTRN12606000120572 } \\
\text { Primary outcome mea- } \\
\text { sure(s): BMI z score }\end{array}$ & \multirow{3}{*}{$\begin{array}{l}\text { No } \\
\text { (last verified: February } \\
\text { 2013) } \\
\text { History of changes: no } \\
\text { documented changes }\end{array}$} & $\begin{array}{l}\text { Primary outcome mea- } \\
\text { sure(s): BMI z score }\end{array}$ & $\begin{array}{l}\text { Primary outcome mea- } \\
\text { sure(s): BMI z score }\end{array}$ \\
\hline & $\begin{array}{l}\text { Secondary outcome } \\
\text { measure(s): } \\
\text { waist circumference, fast- } \\
\text { ing lipids, triglyceride, in- } \\
\text { sulin, and glucose, blood } \\
\text { pres- } \\
\text { sure, health-related qual- } \\
\text { ity of life, body satisfac- } \\
\text { tion, parenting, parental } \\
\text { BMI, eating and activity } \\
\text { behaviours, health belief, } \\
\text { programme evaluation }\end{array}$ & & $\begin{array}{l}\text { Sec- } \\
\text { ondary outcome mea- } \\
\text { sure(s): Program Impact } \\
\text { (Parenting Sense of Com- } \\
\text { petence Scale); Parenting } \\
\text { (Alabama Parenting ques- } \\
\text { tionnaire); health-related } \\
\text { quality of life, waist cir- } \\
\text { cumference, fasting lipids, } \\
\text { triglycerides, insulin, glu- } \\
\text { cose, blood pressure, body } \\
\text { satisfaction, eating and ac- } \\
\text { tivity behaviours, health } \\
\text { belief, programme satis- } \\
\text { faction }\end{array}$ & $\begin{array}{l}\text { Secondary outcome } \\
\text { measure }(s) \text { : waist } \mathrm{z} \text { score }\end{array}$ \\
\hline & $\begin{array}{l}\text { Other outcome measure } \\
\text { (s): - }\end{array}$ & & $\begin{array}{l}\text { Other outcome measure } \\
(s) \text { : - }\end{array}$ & $\begin{array}{l}\text { Other outcome measure } \\
\text { (s): - }\end{array}$ \\
\hline \multirow[t]{3}{*}{ Jansen 2011} & \multirow[t]{3}{*}{ Source: N/T } & & $\begin{array}{l}\text { Primary outcome mea- } \\
\text { sure(s): - }\end{array}$ & $\begin{array}{l}\text { Primary outcome mea- } \\
\text { sure(s): - }\end{array}$ \\
\hline & & & $\begin{array}{l}\text { Secondary outcome } \\
\text { measure(s): - }\end{array}$ & $\begin{array}{l}\text { Secondary } \quad \text { outcome } \\
\text { measure(s): - }\end{array}$ \\
\hline & & & $\begin{array}{l}\text { Outcomes not specified } \\
\text { as primary or secondary: } \\
\text { weight; height; BMI per- } \\
\text { centile, eating psy- } \\
\text { chopathology (Child Eat- } \\
\text { ing Disorders Examina- } \\
\text { tion Questionnaire), eat- } \\
\text { ing behaviours (self re- } \\
\text { port), physical activity } \\
\text { (Baecke Questionnaire), } \\
\text { self esteem (Self-Percep- } \\
\text { tion Profile for Children), } \\
\text { negative thoughts (Heavy } \\
\text { Thoughts Questionnaire) } \\
\text {, knowledge test, motiva- } \\
\text { tion (therapist rated) }\end{array}$ & $\begin{array}{l}\text { Other outcome mea- } \\
\text { sure(s): BMI percentile, } \\
\text { relapse, psychopathology, } \\
\text { self esteem and negative } \\
\text { thoughts }\end{array}$ \\
\hline
\end{tabular}




\begin{tabular}{|c|c|c|c|c|}
\hline \multirow[t]{2}{*}{ Collins 2011} & $\begin{array}{l}\text { Source: NCT00107692 } \\
\text { Primary outcome mea- } \\
\text { sure(s): - }\end{array}$ & \multirow{2}{*}{$\begin{array}{l}\text { No } \\
\text { (last verified: September } \\
\text { 2006) } \\
\text { History of changes: } 5 \\
\text { documented changes }\end{array}$} & $\begin{array}{l}\text { Primary outcome mea- } \\
\text { sure(s): BMI z score and } \\
\text { waist circumference }\end{array}$ & $\begin{array}{l}\text { Primary outcome mea- } \\
\text { sure(s): BMI z score, waist } \\
\text { measurements }\end{array}$ \\
\hline & $\begin{array}{l}\text { Secondary outcome } \\
\text { measure(s): - }\end{array}$ & & $\begin{array}{l}\text { Secondary out- } \\
\text { come measure(s): blood } \\
\text { pressure, cholesterol, C- } \\
\text { reactive protein, triglyc- } \\
\text { erides, glucose, insulin, } \\
\text { energy intake, physical ac- } \\
\text { tivity, movement and skill } \\
\text { proficiency, perceived ath- } \\
\text { letic competence, screen } \\
\text { behaviours }\end{array}$ & $\begin{array}{l}\text { Sec- } \\
\text { ondary outcome mea- } \\
\text { sure(s): metabolic out- } \\
\text { comes }\end{array}$ \\
\hline
\end{tabular}

Outcomes not stated as primary or secondary: BMI SD score, height, weight, and waist circumference; metabolic profile measures: blood pressure; cholesterol, triglycerides; glucose and insulin; physical activity energy expenditure and sedentary activities: total kilocalories expended and time spent in sedentary activities; dietary energy intake: 4-day weighed food record (2 week days and the weekend), parent selection of lower fat items in the household grocery shopping and behaviour changes related to a healthy lifestyle; actual and perceived competence: Test of Gross Motor Development and the Self-Perception Profile for Children. Activity of daily living: Sit-to-stand transfer
Other outcome measure (s): - (s): -
Boutelle 2011 Source: N/T
Primary outcome measure(s): weight, BMI (basis of non-inferiority test)
Primary measure(s): inferiority of treatment group on child weight loss 


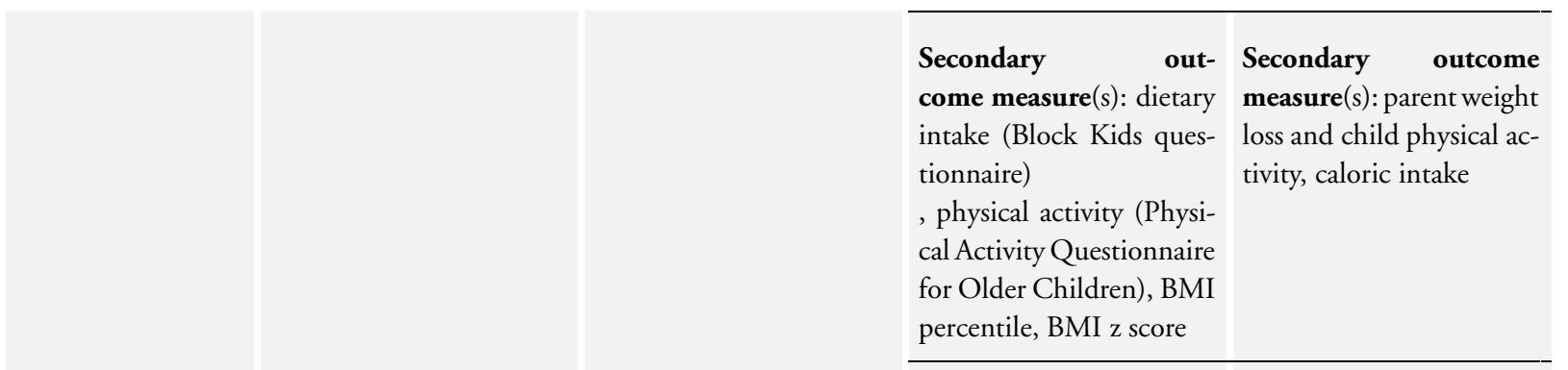

Other outcome measure

(s): -

$$
\text { (s): - }
$$

\begin{tabular}{|c|c|c|c|}
\hline \multirow[t]{2}{*}{ West 2010} & \multirow[t]{2}{*}{ Source: N/T } & $\begin{array}{l}\text { Primary outcome mea- } \\
\text { sure(s): BMI z score }\end{array}$ & $\begin{array}{l}\text { Primary outcome mea- } \\
\text { sure(s): child BMI z score }\end{array}$ \\
\hline & & $\begin{array}{l}\text { Secondary outcome } \\
\text { measure(s) } \\
\text { : weight-related problem } \\
\text { behaviour and parenting } \\
\text { self efficacy (Lifestyle Be- } \\
\text { haviour Checklist); inef- } \\
\text { fective parenting (Parent- } \\
\text { ing Scale) }\end{array}$ & $\begin{array}{l}\text { Sec- } \\
\text { ondary outcome mea- } \\
\text { sure(s): weight-related } \\
\text { problem behaviour, con- } \\
\text { fidence in managing chil- } \\
\text { dren's weight-related be- } \\
\text { haviour }\end{array}$ \\
\hline
\end{tabular}

Other outcome measure Other outcome measure (s): -

$$
\text { (s): - }
$$

Resnick $2009 \quad$ Source: N/T

Primary outcome measure(s): -

\begin{tabular}{lll} 
Secondary & outcome \\
measure(s): - & $\begin{array}{l}\text { Secondary outcome } \\
\text { measure(s): - }\end{array}$ \\
\hline
\end{tabular}

Outcomes not specified as primary or secondary: sources of information, parent confidence, behavioural questions, patient satisfaction (all self report measures), BMI
Other outcome measure

\begin{tabular}{|c|c|c|}
\hline \multirow[t]{2}{*}{ Estabrooks 2009} & $\begin{array}{l}\text { Source: NCT00433901 } \\
\text { Primary outcome mea- } \\
\text { sure(s): child BMI }\end{array}$ & \multirow{2}{*}{$\begin{array}{l}\text { No } \\
\text { (last verified: July 2010) } \\
\text { History of changes: } 2 \\
\text { documented changes }\end{array}$} \\
\hline & $\begin{array}{l}\text { Secondary out- } \\
\text { come measure(s): parent } \\
\text { BMI, objective measure }\end{array}$ & \\
\hline
\end{tabular}
(s): BMI 
of physical activity using child accelerometer, Kids Eating Disorder Survey (KEDS), Peds QOL survey, SE (self efficacy), self report of physical activity, self report of sedentary activity, children's block food frequency survey, parent's home environment survey, parent self efficacy, parent demographics, parent quality of life, parent rapid assessment of physical activity, parent fat and fibre survey, parent health literacy, child/family medical history, economic survey type of medical weight management services

Other outcome measure

(s): -

Source: N/T sure(s): physical activity and sedentary behaviour (Youth Behavioural Risk Survey question); fruit, vegetable and sugared-drink consumption (Block Kids Questionnaire); eating disorder symptoms (Kids Eating Disorders Survey); healthrelated quality of life (Peds QOL survey), self efficacy eating disorders and body image 


\begin{tabular}{|c|c|c|c|c|}
\hline \multirow[t]{3}{*}{ Janicke 2008} & \multirow[t]{3}{*}{ Source: N/T } & & $\begin{array}{l}\text { Primary outcome mea- } \\
\text { sure(s): BMI z score (ba- } \\
\text { sis of power calculation) }\end{array}$ & \multirow[t]{2}{*}{$\begin{array}{l}\text { Primary outcome mea- } \\
\text { sure(s): BMI z score, }\end{array}$} \\
\hline & & & $\begin{array}{l}\text { Secondary outcome } \\
\text { measure(s): Youth/Ado- } \\
\text { lescent Food Frequency } \\
\text { Questionnaire }\end{array}$ & \\
\hline & & & $\begin{array}{l}\text { Other outcome measure } \\
(s):-\end{array}$ & $\begin{array}{l}\text { Other outcome measure } \\
\text { (s): - }\end{array}$ \\
\hline \multirow[t]{3}{*}{ Golley 2007} & $\begin{array}{l}\text { Source: } \\
\text { ACTRN12606000119594 } \\
\text { Primary outcome mea- } \\
\text { sure(s): BMI z score }\end{array}$ & \multirow[t]{3}{*}{$\begin{array}{l}\text { No } \\
\text { (last verified: February } \\
\text { 2013) } \\
\text { History of changes: no } \\
\text { documented changes }\end{array}$} & $\begin{array}{l}\text { Primary outcome mea- } \\
\text { sure(s): BMI z score }\end{array}$ & $\begin{array}{l}\text { Primary outcome mea- } \\
\text { sure(s): BMI z score }\end{array}$ \\
\hline & $\begin{array}{l}\text { Secondary outcome } \\
\text { measure(s): } \\
\text { waist circumference, fast- } \\
\text { ing lipids, triglyceride, in- } \\
\text { sulin, and glucose, blood } \\
\text { pres- } \\
\text { sure, health-related qual- } \\
\text { ity of life, body satisfac- } \\
\text { tion, parental weight sta- } \\
\text { tus and waist circumfer- } \\
\text { ence, eating and activity } \\
\text { behaviours, parental com- } \\
\text { petency, parental satisfac- } \\
\text { tion }\end{array}$ & & $\begin{array}{l}\text { Secondary outcome } \\
\text { measure(s): waist circum- } \\
\text { ference, blood pres- } \\
\text { sure; fasting glucose; to- } \\
\text { tal cholesterol; high-den- } \\
\text { sity lipoprotein choles- } \\
\text { terol; low-density lipopro- } \\
\text { tein cholesterol; triacyl- } \\
\text { glycerol; programme eval- } \\
\text { uation; } \\
\text { satisfaction; health related } \\
\text { quality of life }\end{array}$ & $\begin{array}{l}\text { Secondary outcome } \\
\text { measure }(s) \text { : waist circum- } \\
\text { ference z score }\end{array}$ \\
\hline & $\begin{array}{l}\text { Other outcome measure } \\
\text { (s): - }\end{array}$ & & $\begin{array}{l}\text { Other outcome measure } \\
(s):-\end{array}$ & $\begin{array}{l}\text { Other outcome measure } \\
\text { (s): - }\end{array}$ \\
\hline \multirow[t]{2}{*}{ Golan 2006} & \multirow[t]{2}{*}{ Source: N/T } & & $\begin{array}{l}\text { Primary outcome mea- } \\
\text { sure(s): weight loss (basis } \\
\text { of power calculation) }\end{array}$ & $\begin{array}{l}\text { Primary outcome mea- } \\
\text { sure(s): \% overweight at } \\
\text { end of programme ( } 6 \\
\text { months) and 1-year fol- } \\
\text { low-up }\end{array}$ \\
\hline & & & $\begin{array}{l}\text { Secondary out- } \\
\text { come measure(s): weight; } \\
\text { height, BMI z score; fam- } \\
\text { ily eating questionnaire; } \\
\text { parenting style (Parental } \\
\text { Authority Questionnaire) }\end{array}$ & $\begin{array}{l}\text { Secondary outcome } \\
\text { measure(s): food stimuli } \\
\text { in the home (from Family } \\
\text { Eating and Activity ques- } \\
\text { tionnaire), parent's weight }\end{array}$ \\
\hline
\end{tabular}


Other outcome measure (s): -
Other outcome measure (s): -

Primary outcome mea- Primary outcome measure(s): sure(s): -

$\begin{array}{ll}\begin{array}{l}\text { Secondary outcome } \\ \text { measure(s): - }\end{array} & \begin{array}{l}\text { Secondary outcome } \\ \text { measure(s): - }\end{array}\end{array}$

Outcomes not stated as primary or secondary: weight, height
Other outcome measure (s): weight change

\section{- denotes not reported}

${ }^{a}$ Trial document(s) refers to all available information from published design papers and sources other than regular publications (e.g. FDA/EMA documents, manufacturer's website's, trial registers)

${ }^{b}$ Publication(s) refers to trial information published in scientific journals (primary reference, duplicate publications, companion documents or multiple reports of a primary study)

ACTRN: Australian New Zealand Clinical Trials Registry; BMA: body mass index; EMA: European Medicines Agency; FDA: Food and Drug Administration (US); ISRCTN: International Standard Randomised Controlled Trial Number; LDL: low-density lipoprotein; N/A: not applicable; N/T: no trial document available; TV: television

\section{Appendix 6. Examination of outcome reporting bias according to ORBIT classification}

\begin{tabular}{|c|c|c|c|c|c|}
\hline & Outcome & 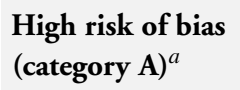 & $\begin{array}{l}\text { High risk of bias } \\
\text { (category D) }^{b}\end{array}$ & 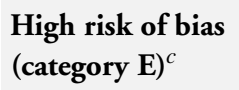 & 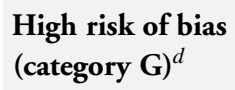 \\
\hline Resnicow 2015 & N/A & & & & \\
\hline Mazzeo 2014 & N/A & & & & \\
\hline van Grieken 2013 & N/A & & & & \\
\hline Small 2013 & N/A & & & & \\
\hline Esfarjani 2013 & N/A & & & & \\
\hline Moens 2012 & N/A & & & & \\
\hline Raynor 2012a & N/A & & & & \\
\hline Raynor 2012b & N/A & & & & \\
\hline
\end{tabular}

Magarey $2011 \quad$ N/A 
(Continued)

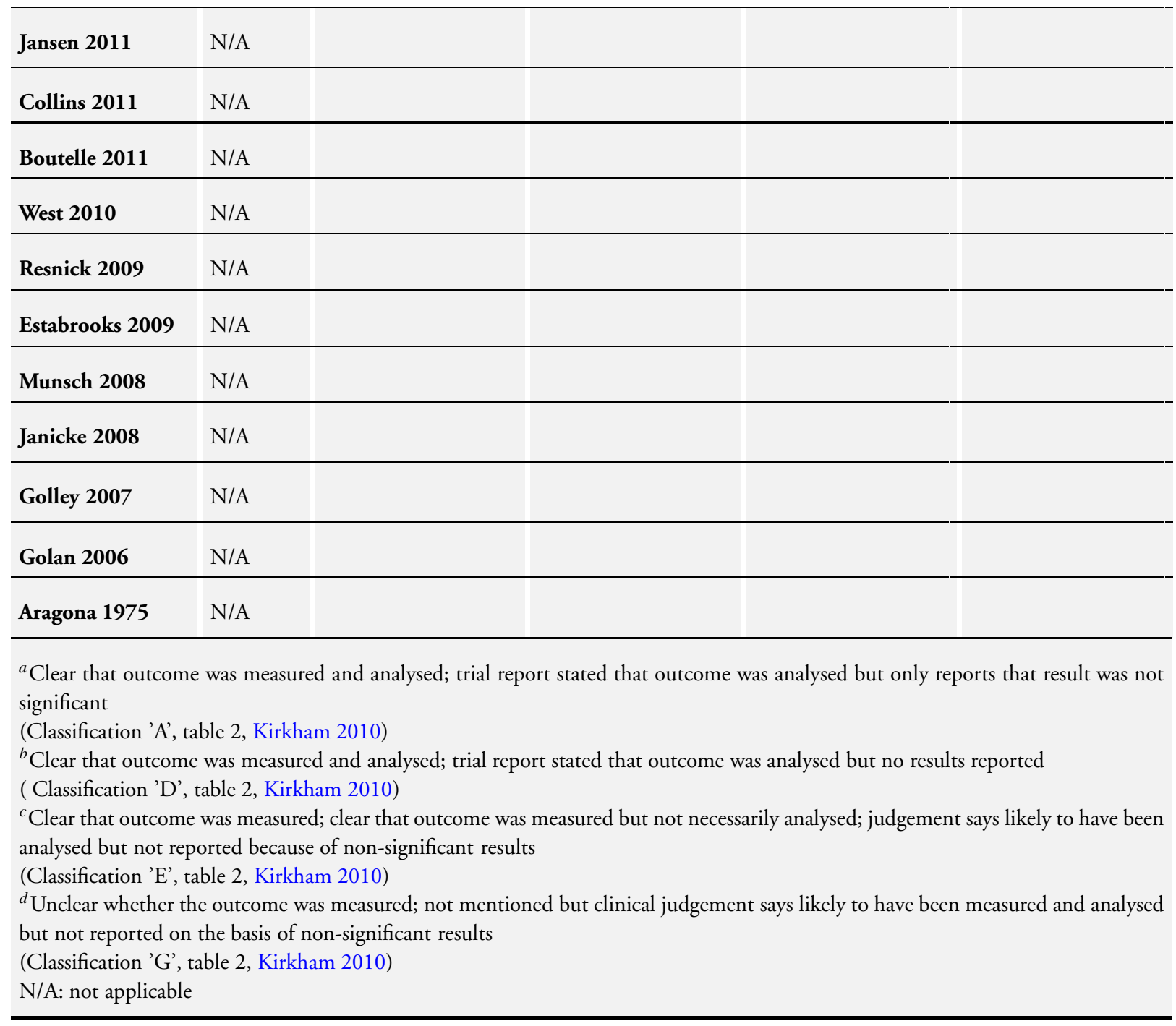

\section{Appendix 7. Definition of endpoint measurement}

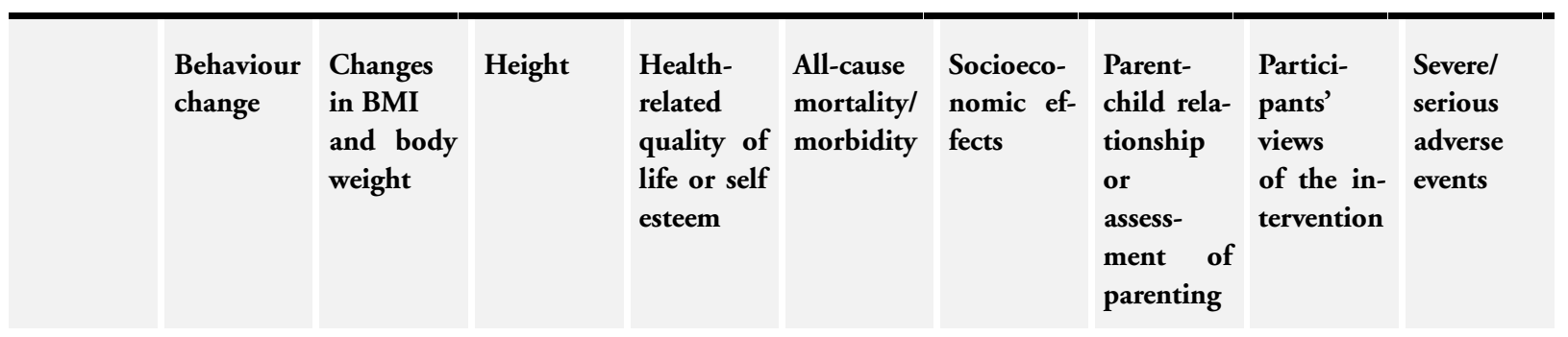




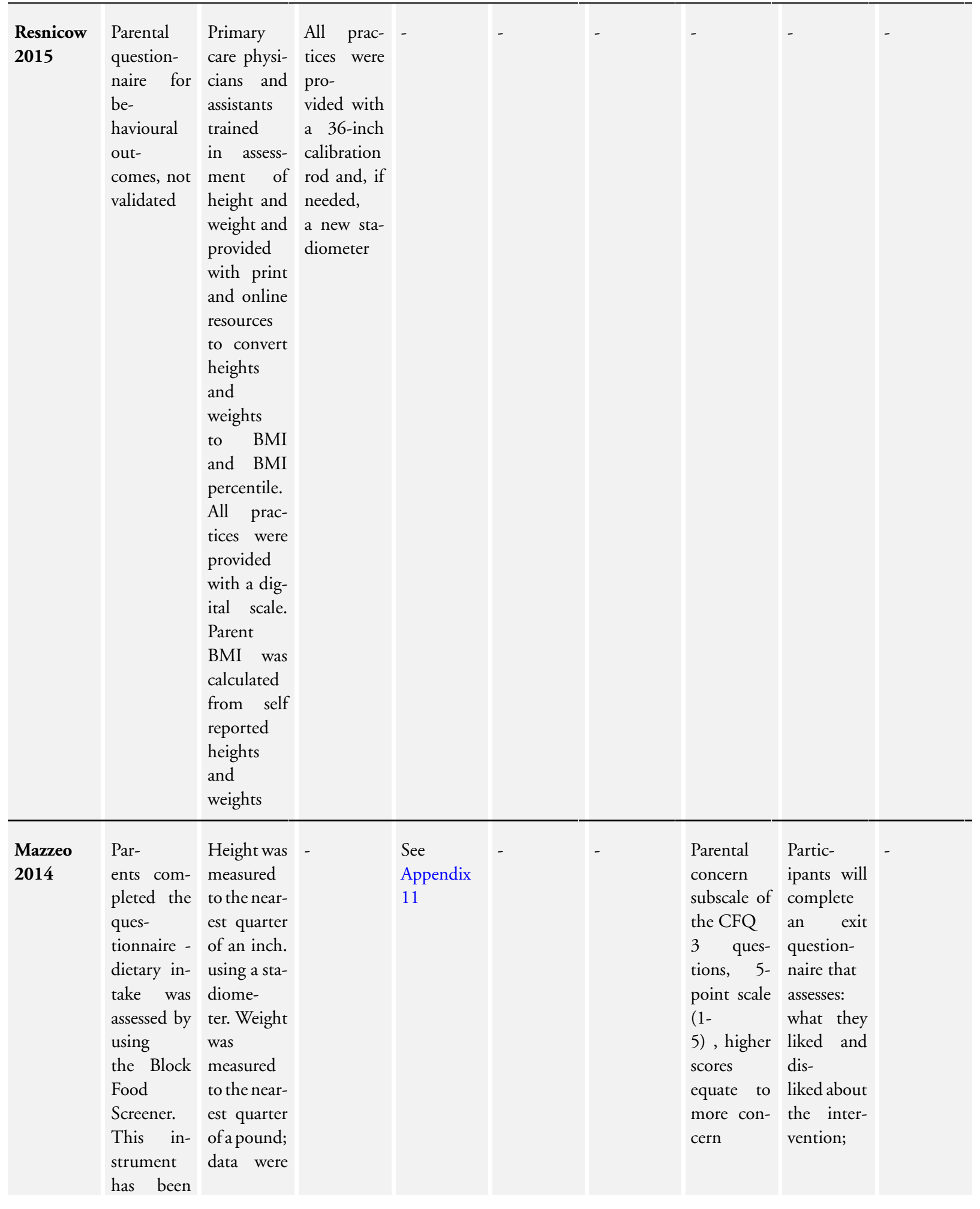




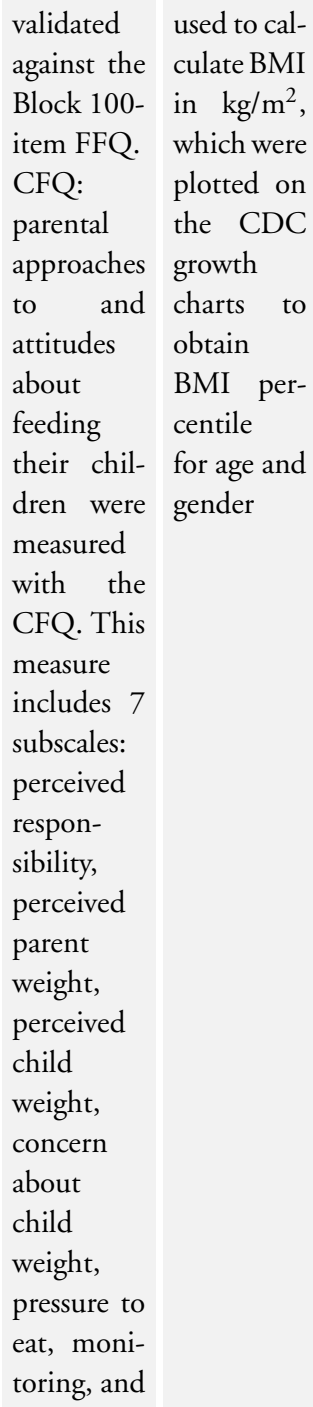

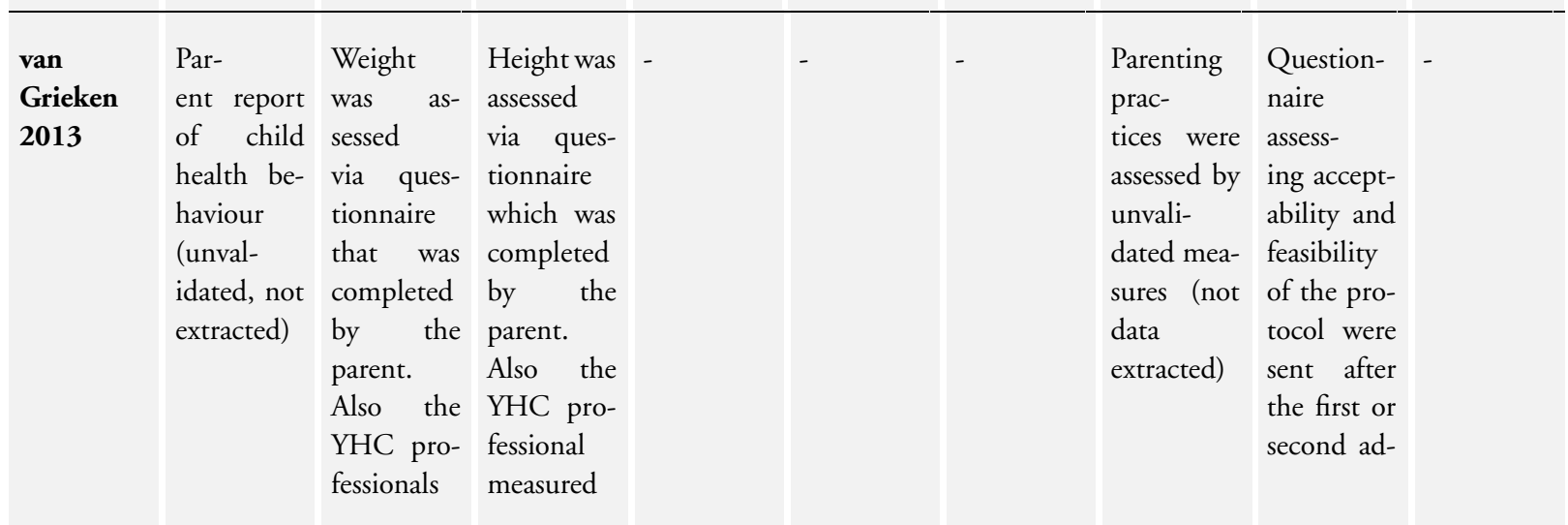

thoughts

about the duration,

frequency, and number of sessions; perceived benefits and barriers to implementing the intervention goals; comfort with group leaders and members; overall satisfaction; and suggestions for improvement 


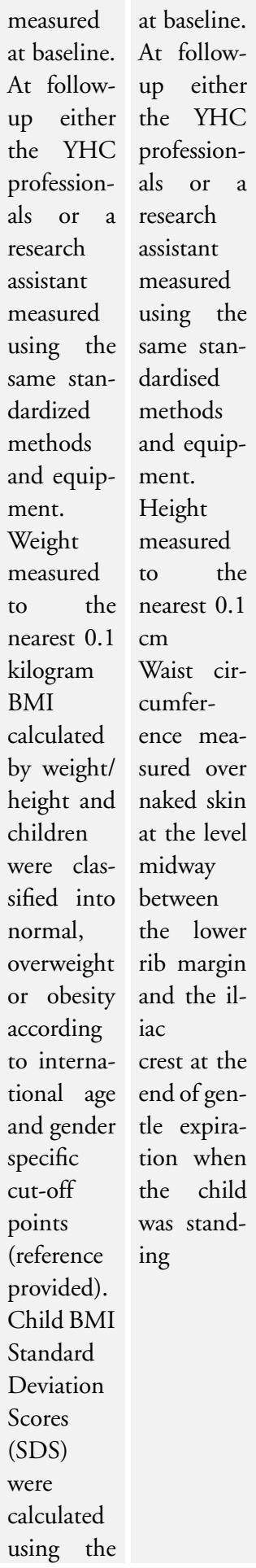

di-

tional ses-

sion. Par-

ents

were asked

to indicate

if the infor-

mation

provided

was appre-

ciated 


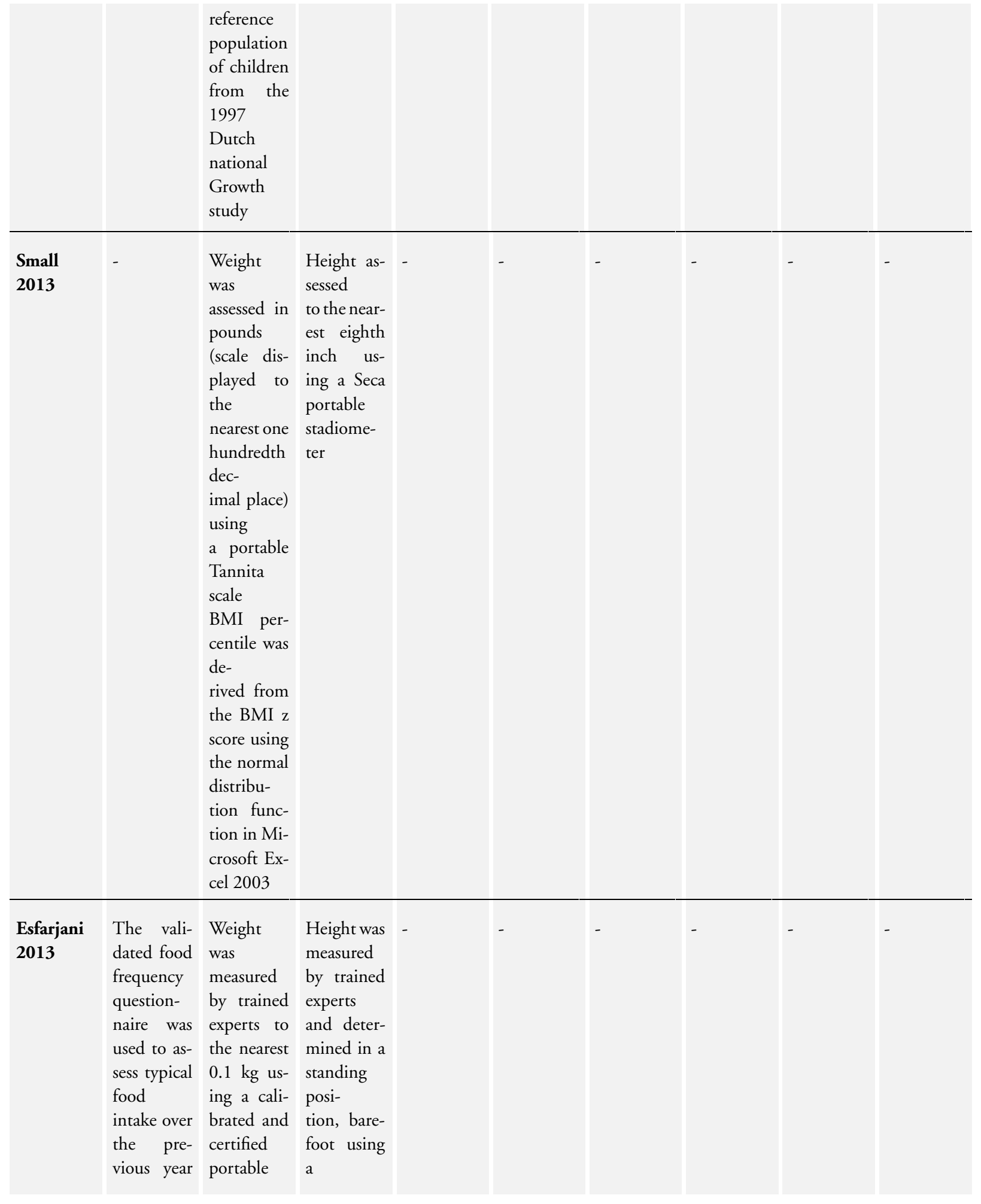




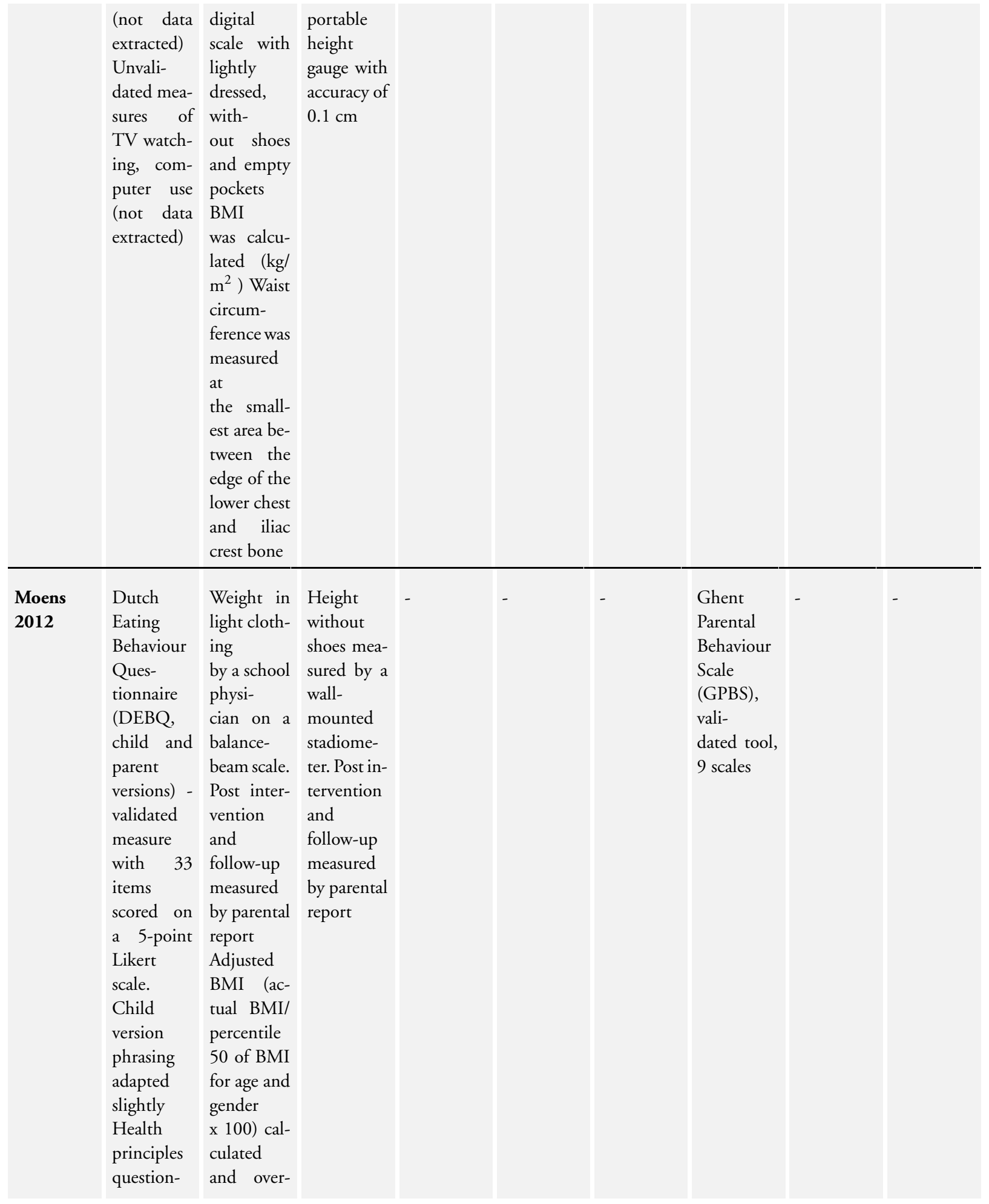




$$
\begin{array}{ll}
\text { naire - de- } & \text { weight or } \\
\text { veloped for } & \text { obese iden- } \\
\text { the study, } & \text { tified in re- } \\
\text { not val- } & \text { lation to } \\
\text { idated (not } & \text { European } \\
\text { data norms for } \\
\text { extracted) } & 0-21 \text { year } \\
& \text { olds. Also } \\
& \text { BMI per- } \\
& \text { centiles } \\
& \text { and } \mathrm{z} \\
& \text { scores cal- } \\
& \text { culated by } \\
& \text { US CDC }
\end{array}
$$

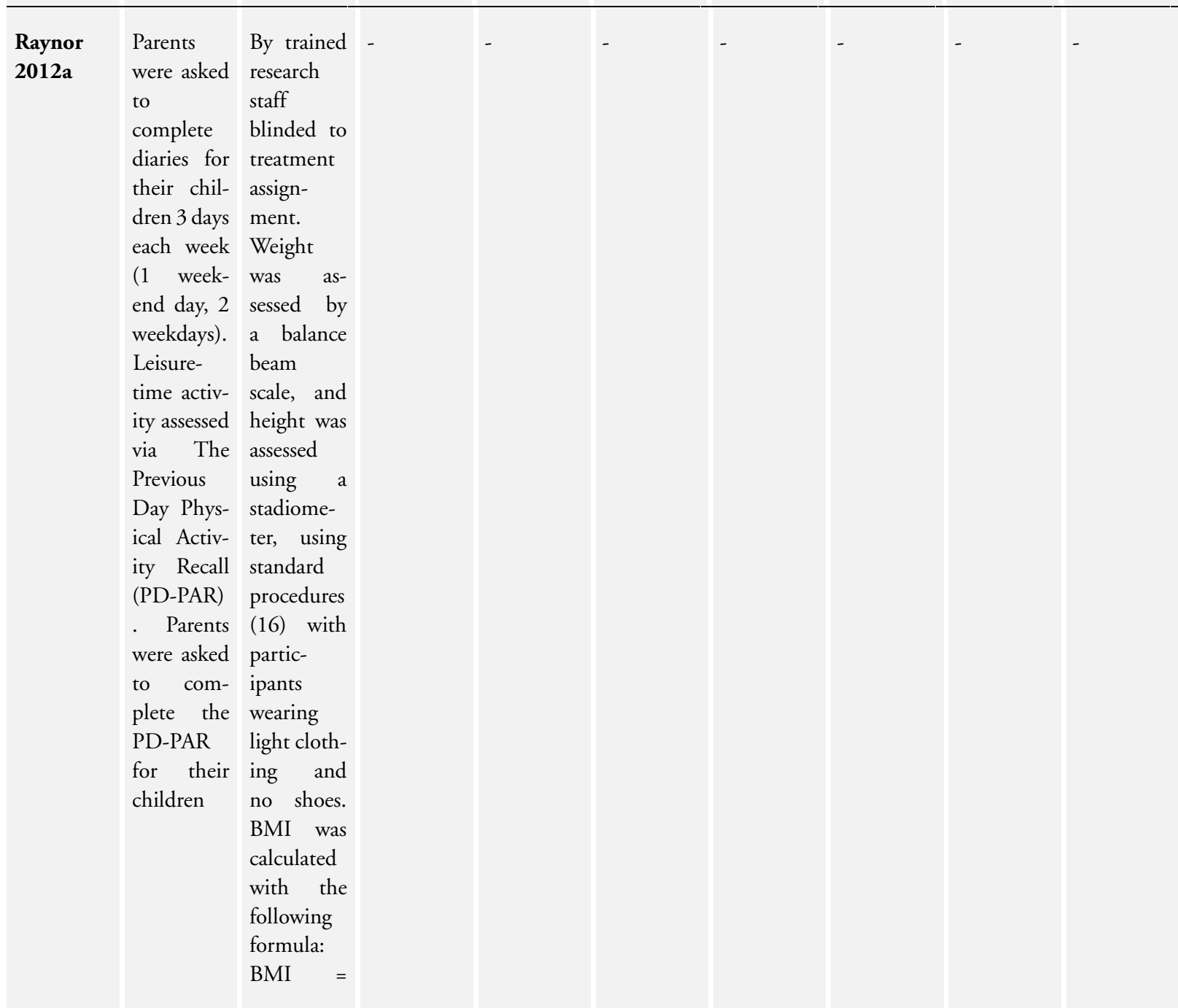




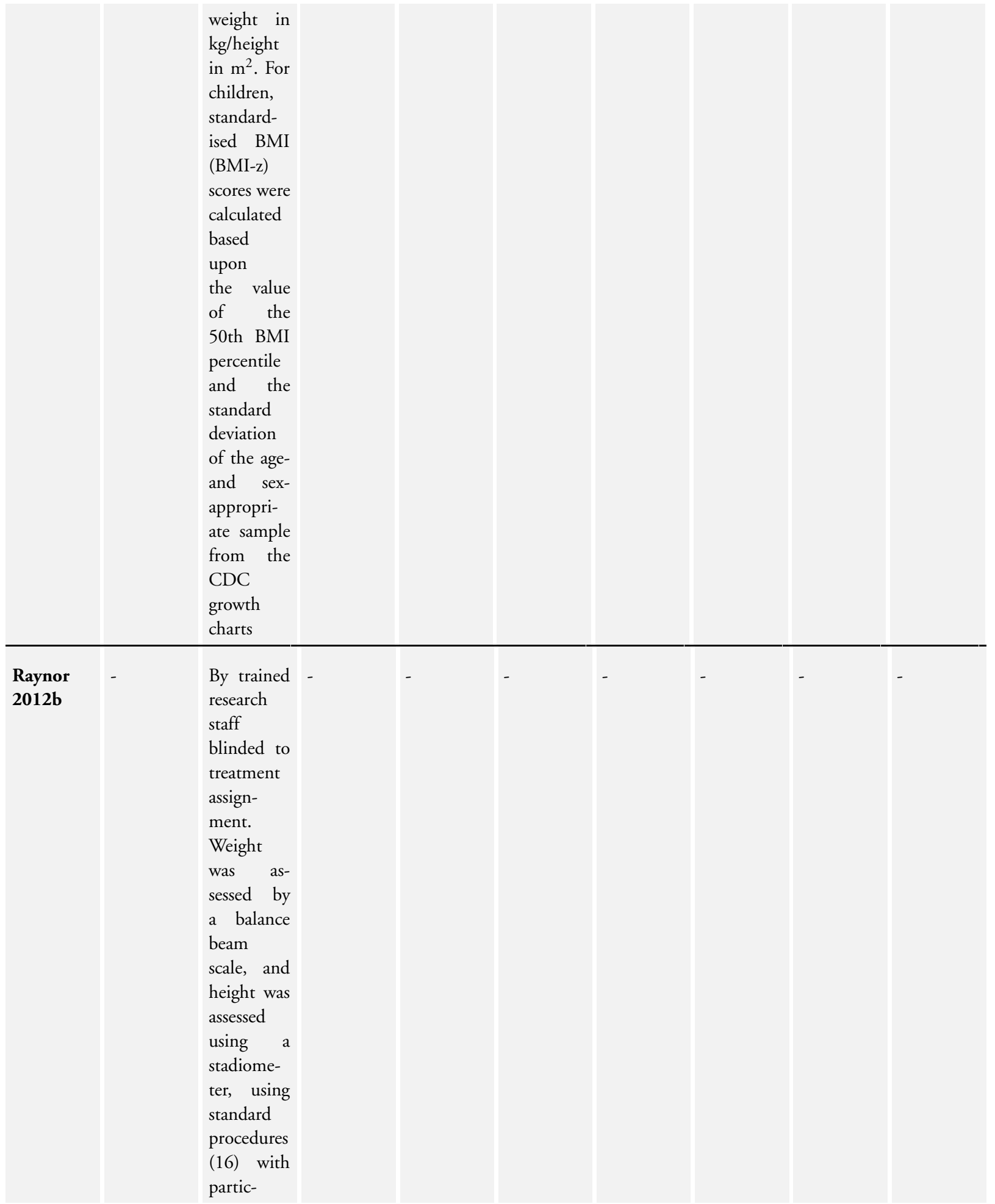

Parent-only interventions for childhood overweight or obesity in children aged 5 to II years (Review) 


$$
\begin{aligned}
& \text { ipants } \\
& \text { wearing } \\
& \text { light cloth- } \\
& \text { ing and } \\
& \text { no shoes. } \\
& \text { BMI was } \\
& \text { calculated } \\
& \text { with the } \\
& \text { following } \\
& \text { formula: } \\
& \text { BMI } \\
& \text { weight in } \\
& \text { kg/height } \\
& \text { in }{ }^{2} \text {. For } \\
& \text { children, } \\
& \text { standard- } \\
& \text { ised BMI } \\
& \text { (BMI-z) } \\
& \text { scores were } \\
& \text { calculated } \\
& \text { based } \\
& \text { upon } \\
& \text { the value } \\
& \text { of the } \\
& 50 \text { th BMI } \\
& \text { percentile } \\
& \text { and the } \\
& \text { standard } \\
& \text { deviation } \\
& \text { of the age- } \\
& \text { and sex- } \\
& \text { appropri- } \\
& \text { ate sample } \\
& \text { from the } \\
& \text { CDC } \\
& \text { growth } \\
& \text { charts }
\end{aligned}
$$

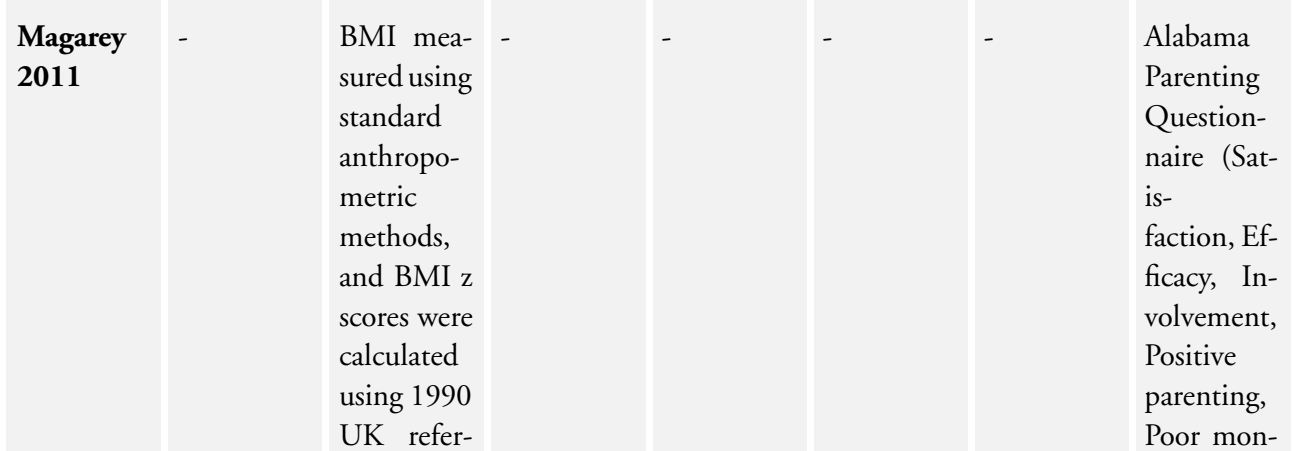




$$
\begin{aligned}
& \text { ence } \\
& \text { data child } \\
& \text { weight sta- } \\
& \text { tus as de- } \\
& \text { ter- } \\
& \text { mined us- } \\
& \text { ing IOTF } \\
& \text { definition }
\end{aligned}
$$

\author{
itoring, In- \\ consis- \\ tent disci- \\ pline, Cor- \\ poral pun-

\begin{tabular}{|c|c|c|c|}
\hline $\begin{array}{l}\text { Jansen } \\
2011\end{array}$ & $\begin{array}{l}\text { A self re- } \\
\text { port ques- } \\
\text { tionnaire. } \\
\text { Child Eat- } \\
\text { ing Disor- } \\
\text { der Exami- } \\
\text { na- } \\
\text { tion Ques- } \\
\text { tionnaire, } \\
\text { which } \\
\text { is based on } \\
\text { the Eating } \\
\text { Disorder } \\
\text { Examina- } \\
\text { tion Ques- } \\
\text { tionnaire. } \\
\text { Eating be- } \\
\text { haviour by } \\
\text { interview, } \\
\text { Scores for } \\
\text { regu- } \\
\text { larity range } \\
\text { from 0 to } \\
7 \text {, a higher } \\
\text { score indi- } \\
\text { cating } \\
\text { a more reg- } \\
\text { ular eating } \\
\text { pattern. } \\
\text { Physical } \\
\text { activity: } \\
\text { filled } \\
\text { out by } \\
\text { overweight } \\
\text { child (in } \\
\text { presence }\end{array}$ & $\begin{array}{l}\text { Weight } \\
\text { (kg) mea- } \\
\text { sured and } \\
\text { BMI per- } \\
\text { centile } \\
\text { (Children's } \\
\text { BMI-for- } \\
\text { Age Calcu- } \\
\text { lator) was } \\
\text { cal- } \\
\text { culated for } \\
\text { each child } \\
\text { of } \\
\text { the partici- } \\
\text { pating par- } \\
\text { ents, thus } \\
\text { in- } \\
\text { cluding the } \\
\text { target chil- } \\
\text { dren and } \\
\text { their } \\
\text { siblings }\end{array}$ & $\begin{array}{l}\text { Self es- } \\
\text { teem: } \\
\text { overweight } \\
\text { child filled } \\
\text { out the } \\
\text { question- } \\
\text { naire (in } \\
\text { presence } \\
\text { of a parent } \\
\text { and the in- } \\
\text { terviewer) } \\
\text {. Perceived } \\
\text { compe- } \\
\text { tence was } \\
\text { measured } \\
\text { with the } \\
\text { translated } \\
\text { version of } \\
\text { the Self- } \\
\text { Perception } \\
\text { Profile for } \\
\text { Children. } \\
\text { Negative } \\
\text { thoughts: } \\
\text { completed } \\
\text { by the } \\
\text { overweight } \\
\text { child (in } \\
\text { presence of } \\
\text { a parent } \\
\text { and the in- } \\
\text { terviewer). } \\
\text { This ques- } \\
\text { tion- }\end{array}$ \\
\hline
\end{tabular} \\ ishment) \\ 35 \\ questions, \\ higher \\ scores in- \\ dicate im- \\ provement
}




\begin{tabular}{|c|c|}
\hline $\begin{array}{l}\text { of a parent } \\
\text { and the in- } \\
\text { terviewer). } \\
\text { This ques- } \\
\text { tionnaire } \\
\text { consists } \\
\text { of } 3 \text { com- } \\
\text { ponents: } \\
\text { work activ- } \\
\text { ity, sports } \\
\text { activity } \\
\text { and leisure } \\
\text { activity. } \\
\text { An index } \\
\text { score for } \\
\text { the } 3 \text { com- } \\
\text { ponents is } \\
\text { calculated, } \\
\text { ranging } \\
\text { from } 1 \text { to } \\
5 \text {, with } \\
\text { higher in- } \\
\text { dex scores } \\
\text { reflecting } \\
\text { higher } \\
\text { levels of } \\
\text { activ- } \\
\text { ity. The } \\
\text { activity' } \\
\text { (including } \\
\text { similar } \\
\text { questions) } \\
\text { Question- } \\
\text { naire was } \\
\text { adapted } \\
\text { for use } \\
\text { with chil- } \\
\text { dren by } \\
\text { replacing } \\
\text { work ac- } \\
\text { alth } \\
\text { and }\end{array}$ & $\begin{array}{l}\text { naire maps } \\
\text { the fre- } \\
\text { quency of } \\
\text { negative } \\
\text { weight- } \\
\text { related } \\
\text { thoughts } \\
\text { in over- } \\
\text { weight } \\
\text { children. } \\
\text { The ques- } \\
\text { tionnaire } \\
\text { comprises } \\
20 \text { items } \\
\text { (for exam- } \\
\text { ple 'I am } \\
\text { worthless } \\
\text { because I } \\
\text { am over- } \\
\text { weight') } \\
\text {. Scores } \\
\text { range from } \\
1 \text { ('I never } \\
\text { have this } \\
\text { thought') } \\
\text { to } 5 \text { ('I } \\
\text { always } \\
\text { have this } \\
\text { thought') }\end{array}$ \\
\hline
\end{tabular}

\begin{tabular}{|c|c|c|c|}
\hline $\begin{array}{l}\text { Collins } \\
2011\end{array}$ & $\begin{array}{l}\text { Habitual } \\
\text { physical } \\
\text { activ- } \\
\text { ity was } \\
\text { measured }\end{array}$ & $\begin{array}{l}\text { Weight ass } \\
\text { mea- } \\
\text { sured with } \\
\text { the chil- } \\
\text { dren bare- }\end{array}$ & $\begin{array}{l}\text { Height was } \\
\text { mea- } \\
\text { sured to } 0 . \\
1 \mathrm{~cm} \text { using } \\
\text { the stretch }\end{array}$ \\
\hline
\end{tabular}




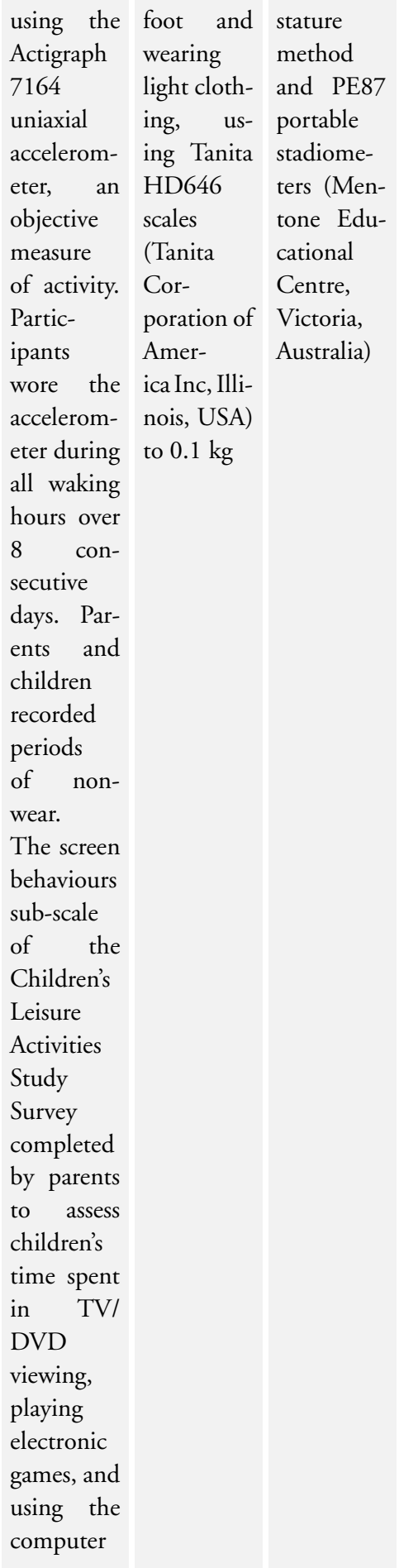




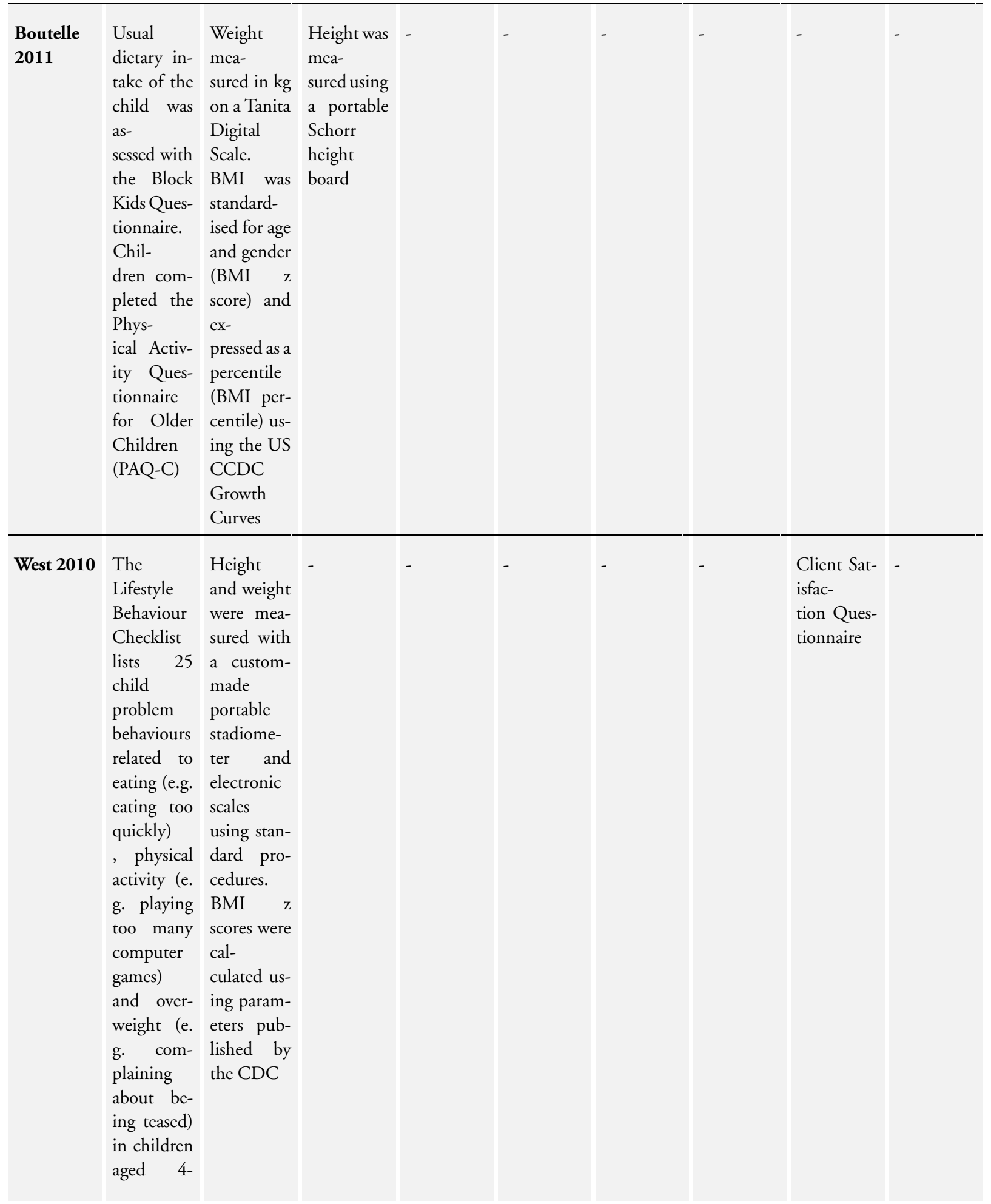




\author{
11 years \\ and yields \\ scores on 2 \\ scales - the \\ Problem \\ scale and \\ Confi- \\ dence \\ scale. \\ For the \\ Problem \\ scale, \\ parents are \\ asked to \\ rate the \\ extent to \\ which they \\ experience \\ each of \\ these \\ behaviours \\ as a prob- \\ lem with \\ their child \\ (higher \\ scores \\ indicate \\ greater \\ problems) \\ . For the \\ Confi- \\ dence \\ scale, \\ parents are \\ asked to \\ rate how \\ confident \\ they feel \\ managing \\ each of \\ these be- \\ haviours, \\ even if not \\ currently \\ occurring \\ (higher \\ scores \\ indicate
}




greater
confi-
dence)
recom-
rece
mended
clinical
cut-offs are
a Problem
scale score
> 50
(range 25-
175) and a
confidence
scale score
< 204
(range $25-$
250 ).
The Par-
enting
Scale: for
each of the
30 items,
parents are
asked to
rate how
they would
respond
to a given
discipline
situation
by choos-
ing be-
tween an
effective or
ineffective
course of
action.
The in-
effective
parenting
practices
assessed by
the Prob-
lem scale
include




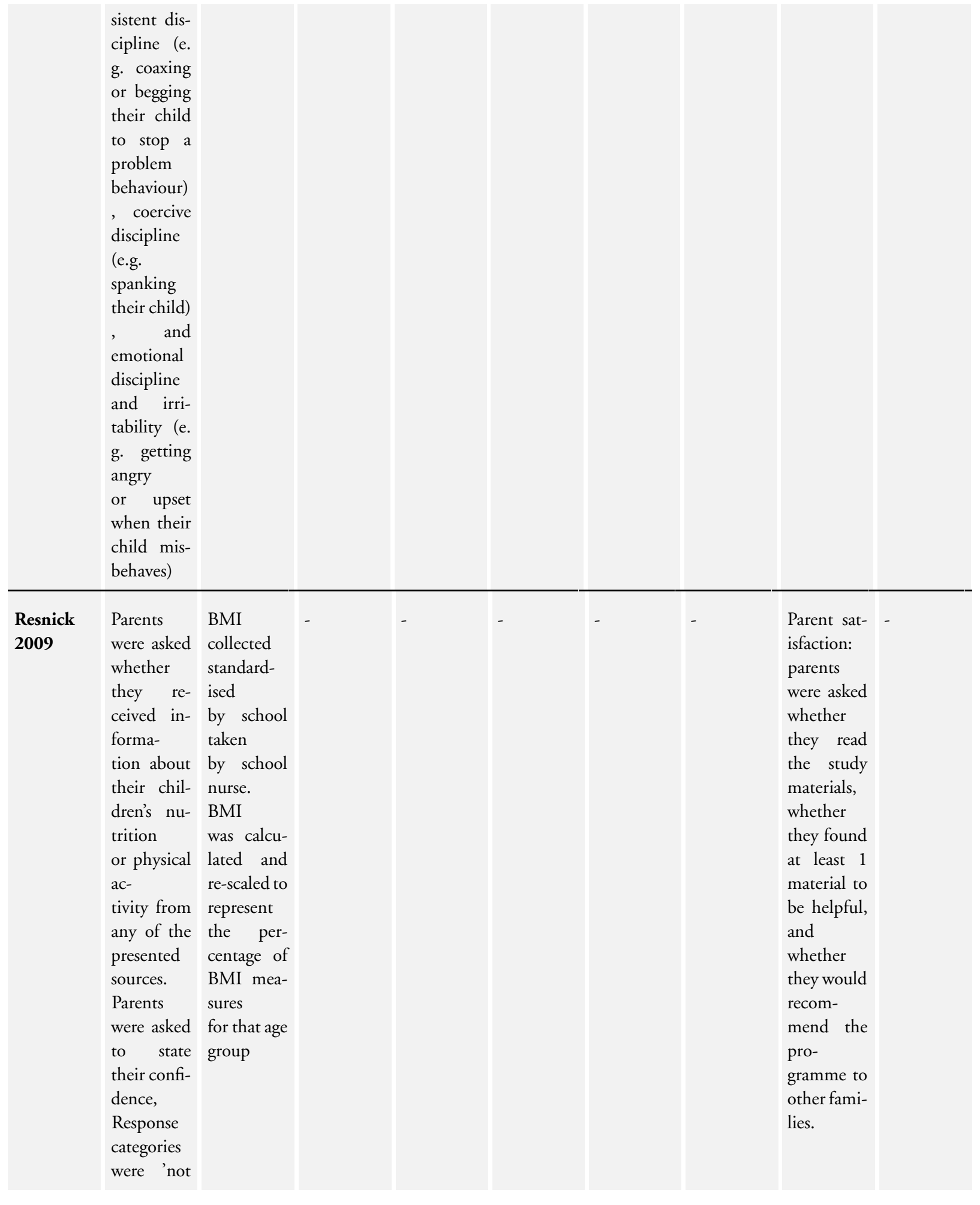

Parent-only interventions for childhood overweight or obesity in children aged 5 to II years (Review) 
at al

confident', 'a little confident', 'somewhat confident', 'confi-

dent',

or 'very confident'.

These

responses

were col-

lapsed into

dichoto-

mous cat-

egories of

confident

and very

confident

vs. not at

all con-

fident, a

little confi-

dent, and

somewhat

confident.

Parents

were asked

to report

the mean number of

hours per

day that

both they

and their

children

watched

TV

videos

during the

30 days

prior to

complet-

ing the baseline

and post-

inter-

Parents in

the educa-

tional ma-

terial plus

personal

encoun-

ters (inter-

vention 2)

group were

also asked

whether

they found

their com-

mu-

nity health

workers to

be helpful 


$$
\begin{aligned}
& \text { vention } \\
& \text { surveys. } \\
& \text { Parents } \\
& \text { were also } \\
& \text { asked } \\
& \text { about the } \\
& \text { number of } \\
& \text { servings of } \\
& \text { fruits and } \\
& \text { vegetables } \\
& \text { that both } \\
& \text { they and } \\
& \text { their } \\
& \text { children } \\
& \text { consumed } \\
& \text { during the } \\
& \text { 30 days } \\
& \text { prior to } \\
& \text { complet- } \\
& \text { ing the } \\
& \text { baseline } \\
& \text { and post- } \\
& \text { inter- } \\
& \text { vention } \\
& \text { surveys. } \\
& \text { Both TV } \\
& \text { and nutri- } \\
& \text { tional } \\
& \text { responses } \\
& \text { were col- } \\
& \text { lapsed into } \\
& \text { dichoto- } \\
& \text { mous cat- } \\
& \text { egories of } \\
& \text { those who } \\
& \text { con- } \\
& \text { sumed } \geq 5 \\
& \text { servings } \\
& \text { of fruit and } \\
& \text { vegetables } \\
& \text { per day vs. } \\
& \text { con- } \\
& \text { sumed } 5 \\
& \text { servings }
\end{aligned}
$$

\begin{tabular}{|c|c|c|}
\hline & - & Weight \\
\hline $\begin{array}{l}\text { tabrooks } \\
2009\end{array}$ & & $\begin{array}{ll}\text { was } & \text { as- } \\
\text { sessed } & \text { us- }\end{array}$ \\
\hline
\end{tabular}


ing a reg-

ularly cali-

brated

medical

scale

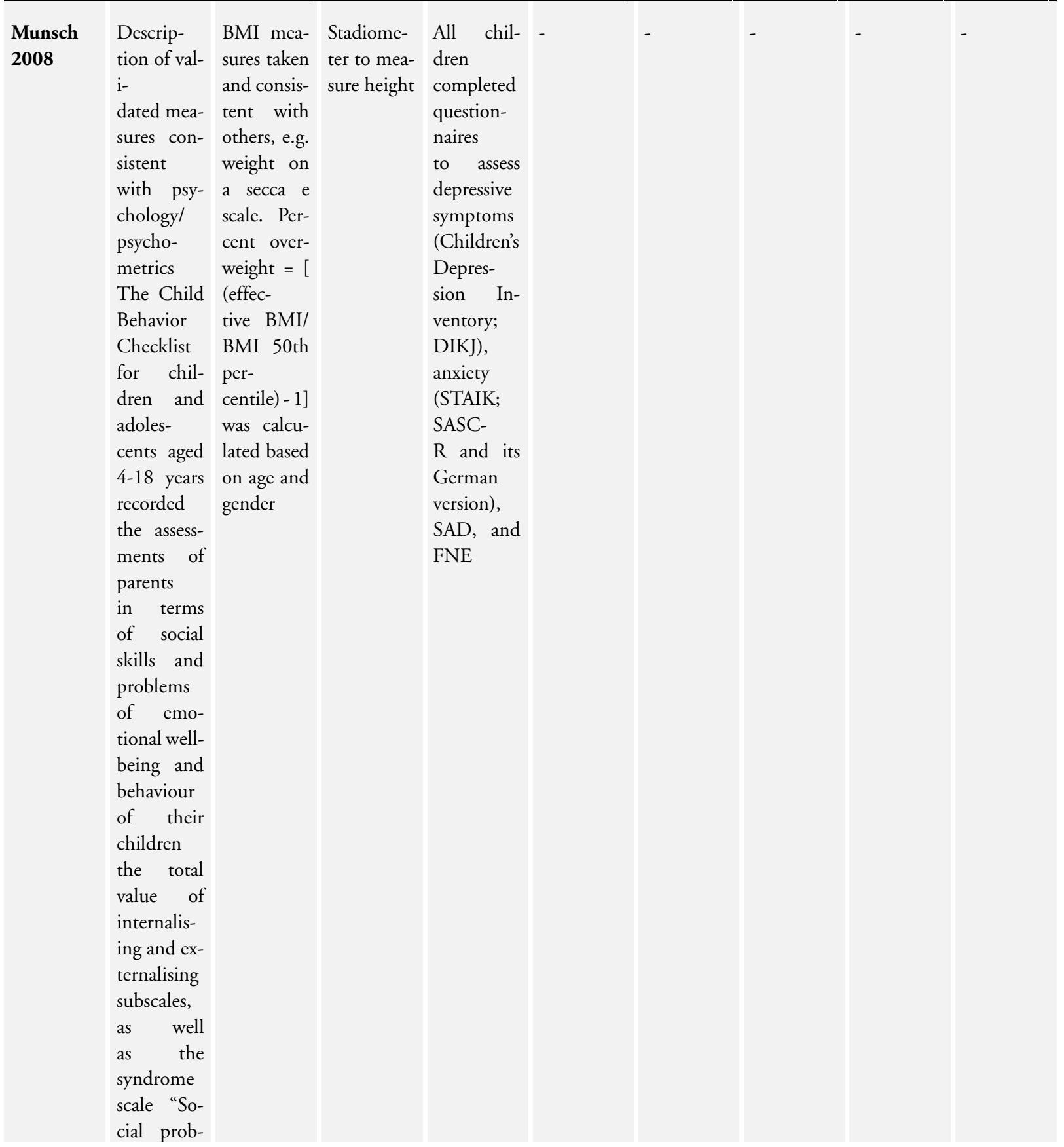




\begin{tabular}{|c|c|c|c|c|c|c|c|c|c|}
\hline & $\begin{array}{l}\text { lems" were } \\
\text { recorded, } \\
\text { repre- } \\
\text { senting a } \\
\text { relevant } \\
\text { concept } \\
\text { in obese } \\
\text { children }\end{array}$ & & & & & & & & \\
\hline $\begin{array}{l}\text { Janicke } \\
2008\end{array}$ & $\begin{array}{l}\text { Parents } \\
\text { were } \\
\text { asked to } \\
\text { help their } \\
\text { children } \\
\text { complete } \\
\text { the mea- } \\
\text { sure The } \\
\text { Youth/ } \\
\text { Adolescent } \\
\text { Food } \\
\text { Frequency } \\
\text { Ques- } \\
\text { tionnaire } \\
\text { was used } \\
\text { to assess } \\
\text { the child's } \\
\text { dietary } \\
\text { intake } \\
\text { during the } \\
\text { preceding } \\
\text { month }\end{array}$ & $\begin{array}{l}\text { Height } \\
\text { without } \\
\text { shoes mea- } \\
\text { sured to } \\
\text { the } \\
\text { nearest } 0.1 \\
\text { cm using a } \\
\text { Harp- } \\
\text { endon sta- } \\
\text { diome- } \\
\text { ter. Weight } \\
\text { to nearest } \\
0.1 \text { kg with } \\
1 \text { layer of } \\
\text { cloth- } \\
\text { ing on and } \\
\text { without } \\
\text { shoes using } \\
\text { a cali- } \\
\text { brated bal- } \\
\text { ance bean } \\
\text { scale. Mea- } \\
\text { sured } 3 \\
\text { times and } \\
\text { averaged }\end{array}$ & $\begin{array}{l}\text { Mea- } \\
\text { sured with- } \\
\text { out shoes } \\
\text { to } \\
\text { nearest } 0.1 \\
\text { cm using a } \\
\text { Harp- } \\
\text { endon sta- } \\
\text { diometer } \\
\text { (Holtain } \\
\text { Ltd, Cross- } \\
\text { well, UK) } \\
\text { Measured } \\
3 \\
\text { times and } \\
\text { averaged }\end{array}$ & $\begin{array}{l}\text { Children } \\
\text { com- } \\
\text { pleted, } \\
\text { Self-Per- } \\
\text { ception } \\
\text { Profile for } \\
\text { Children, } \\
\text { which is a } \\
\text { self report } \\
\text { assessment } \\
\text { of the } \\
\text { child's } \\
\text { perception } \\
\text { of his or } \\
\text { her global } \\
\text { self worth } \\
\text { and com- } \\
\text { petence in } \\
6 \text { specific } \\
\text { domains: } \\
\text { scholastic } \\
\text { compe- } \\
\text { tence, } \\
\text { social ac- } \\
\text { ceptance, } \\
\text { athletic } \\
\text { compe- } \\
\text { tence, } \\
\text { physical } \\
\text { appear- } \\
\text { ance, be- } \\
\text { havioural } \\
\text { conduct, } \\
\text { and global } \\
\text { self worth }\end{array}$ & - & - & - & - & - \\
\hline $\begin{array}{l}\text { Golley } \\
2007\end{array}$ & - & $\begin{array}{l}\text { Weight } \\
\text { was mea- } \\
\text { sured to } \\
\text { the nearest } \\
0.1 \mathrm{~kg} \text { with }\end{array}$ & - & - & - & - & - & $\begin{array}{l}\text { Validated, } \\
\text { anony- } \\
\text { mous 16- } \\
\text { item ques- }\end{array}$ & - \\
\hline
\end{tabular}




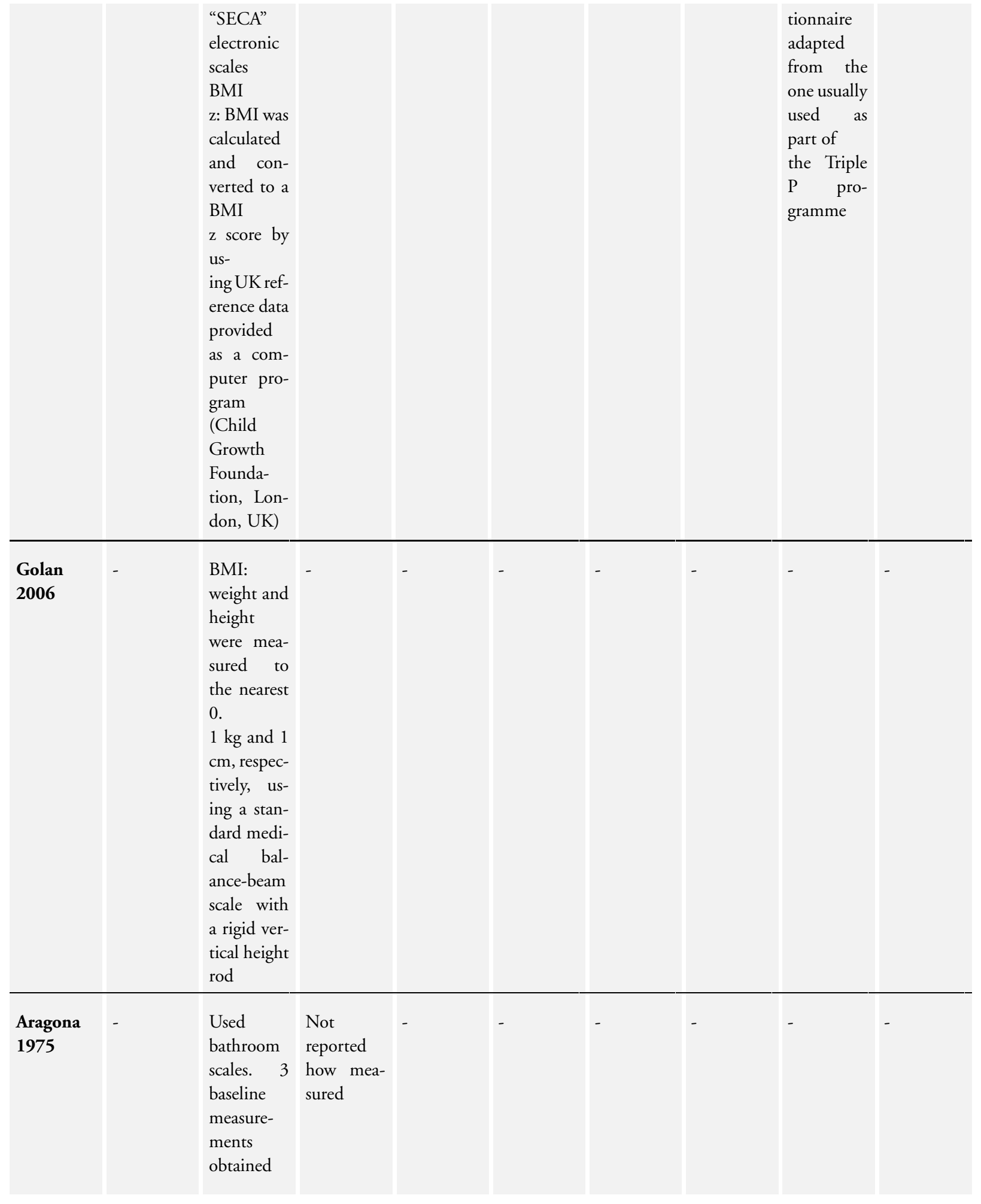




$$
\begin{aligned}
& \text { over a } \\
& \text { 2-week } \\
& \text { period for } \\
& \text { both ex- } \\
& \text { perimental } \\
& \text { groups. } \\
& \text { Control } \\
& \text { group were } \\
& \text { measured } \\
& \text { at home } \\
& \text { on the } \\
& \text { same day. } \\
& \text { To ensure } \\
& \text { reliability } \\
& \text { the exper- } \\
& \text { imenters } \\
& \text { indepen- } \\
& \text { dently read } \\
& \text { all height } \\
& \text { and weight } \\
& \text { measure- } \\
& \text { ments } \\
& \text { in the } \\
& \text { presence } \\
& \text { of the } \\
& \text { parents. } \\
& \text { If there } \\
& \text { were any } \\
& \text { inter-rater } \\
& \text { discrep- } \\
& \text { ancies on } \\
& \text { weight, the } \\
& \text { child was } \\
& \text { weighed } \\
& \text { again. } \\
& \text { Wecutive } \\
& \text { measure- } \\
& \text { recorded } \\
& \text { when } \\
& \text { there was } \\
& \text { inter-rater } \\
& \text { agreement } \\
& \text { on con- }
\end{aligned}
$$

I


PAR SDS: Previous Day Physical Activity Recall; SAD: Social Avoidance and Distress; SASC-R: Social Anxiety Scale for ChildrenRevise; STAIK: State und Trait Angst-Inventar für Kinder; TV: television; YHC: Youth Health Centre

Appendix 8. Adverse events

\begin{tabular}{|c|c|c|c|c|c|c|c|c|c|}
\hline & $\begin{array}{l}\text { Interven- } \\
\text { tion(s) } \\
\text { and } \\
\text { compara- } \\
\text { tor(s) }\end{array}$ & $\begin{array}{l}\text { Partici- } \\
\text { pants } \\
\text { included } \\
\text { in analysis } \\
{[\mathrm{N}]}\end{array}$ & $\begin{array}{l}\text { Deaths } \\
{[N(\%)]}\end{array}$ & $\begin{array}{l}\text { Partici- } \\
\text { pants with } \\
\text { adverse } \\
\text { events } \\
{[\mathrm{N}(\%)]}\end{array}$ & $\begin{array}{l}\text { Partici- } \\
\text { pants with } \\
\text { severe/ } \\
\text { serious } \\
\text { adverse } \\
\text { events } \\
{[\mathrm{N}(\%)]}\end{array}$ & $\begin{array}{l}\text { Partic- } \\
\text { ipants dis- } \\
\text { continu- } \\
\text { ing } \\
\text { study due } \\
\text { to adverse } \\
\text { events } \\
{[\mathrm{N} / \%]}\end{array}$ & $\begin{array}{l}\text { Partici- } \\
\text { pants } \\
\text { hospi- } \\
\text { talised } \\
{[N(\%)]}\end{array}$ & $\begin{array}{l}\text { Partici- } \\
\text { pants with } \\
\text { outpa- } \\
\text { tient treat- } \\
\text { ment } \\
{[\mathrm{N}(\%)]}\end{array}$ & $\begin{array}{l}\text { Partici- } \\
\text { pants with } \\
\text { specific } \\
\text { adverse } \\
\text { events } \\
\text { [descrip- } \\
\text { tion] } \\
{[\mathrm{N} / \%]}\end{array}$ \\
\hline \multirow[t]{3}{*}{$\begin{array}{l}\text { Resnicow } \\
2015\end{array}$} & $\begin{array}{l}\text { I1: parent- } \\
\text { only PCP } \\
\text { motiva- } \\
\text { tional in- } \\
\text { terviewing }\end{array}$ & $\begin{array}{l}16 \\
\text { practices } \\
212 \text { partic- } \\
\text { ipants }\end{array}$ & - & - & - & - & - & - & - \\
\hline & $\begin{array}{l}\text { I2: parent- } \\
\text { only PCP } \\
+ \text { dietician } \\
\text { motiva- } \\
\text { tional in- } \\
\text { terviewing }\end{array}$ & $\begin{array}{l}15 \\
\text { practices } \\
235 \text { partic- } \\
\text { ipants }\end{array}$ & - & - & - & - & - & - & - \\
\hline & $\begin{array}{l}\text { C: usual } \\
\text { care }\end{array}$ & $\begin{array}{l}11 \\
\text { practices } \\
198 \text { partic- } \\
\text { ipants }\end{array}$ & - & - & - & - & - & - & - \\
\hline \multirow[t]{2}{*}{$\begin{array}{l}\text { Mazzeo } \\
2014\end{array}$} & $\begin{array}{l}\text { I: parent } \\
\text { NOUR- } \\
\text { ISH }\end{array}$ & 48 & - & - & - & - & - & - & - \\
\hline & $\begin{array}{l}\text { C: parent } \\
\text { control }\end{array}$ & 45 & - & - & - & - & - & - & - \\
\hline \multirow{2}{*}{$\begin{array}{l}\text { van } \\
\text { Grieken } \\
2013\end{array}$} & $\begin{array}{l}\text { I: parent- } \\
\text { only }\end{array}$ & 349 & - & - & - & - & - & - & - \\
\hline & $\begin{array}{l}\text { C: usual } \\
\text { care }\end{array}$ & 288 & - & - & - & - & - & - & - \\
\hline
\end{tabular}


(Continued)

\begin{tabular}{|c|c|c|c|c|c|c|c|c|c|}
\hline $\begin{array}{l}\text { Small } \\
2013\end{array}$ & $\begin{array}{l}\text { I: parent- } \\
\text { only }\end{array}$ & 34 & - & - & - & - & - & - & - \\
\hline & C: control & 33 & - & - & - & - & - & - & - \\
\hline \multirow[t]{2}{*}{$\begin{array}{l}\text { Esfarjani } \\
2013\end{array}$} & $\begin{array}{l}\text { I: parent- } \\
\text { only }\end{array}$ & 70 & - & - & - & - & - & - & - \\
\hline & C: control & 86 & - & - & - & - & - & - & - \\
\hline \multirow[t]{2}{*}{$\begin{array}{l}\text { Moens } \\
2012\end{array}$} & $\begin{array}{l}\text { I: parent- } \\
\text { only }\end{array}$ & 27 & - & - & - & - & - & - & - \\
\hline & C: control & 19 & - & - & - & - & - & - & - \\
\hline \multirow[t]{3}{*}{$\begin{array}{l}\text { Raynor } \\
2012 a\end{array}$} & $\begin{array}{l}\text { I: parent- } \\
\text { only }\end{array}$ & 33 & - & - & - & - & - & - & - \\
\hline & $\begin{array}{l}\text { C1: parent } \\
\text { - diet de- } \\
\text { crease }\end{array}$ & 33 & - & - & - & - & - & - & - \\
\hline & $\begin{array}{l}\text { C2: parent } \\
\text { - diet in- } \\
\text { crease }\end{array}$ & 35 & - & - & - & - & - & - & - \\
\hline \multirow[t]{3}{*}{$\begin{array}{l}\text { Raynor } \\
2012 b\end{array}$} & $\begin{array}{l}\text { I: parent- } \\
\text { only }\end{array}$ & 29 & - & - & - & - & - & - & - \\
\hline & $\begin{array}{l}\text { C1: parent } \\
\text { - diet and } \\
\text { activity } \\
\text { traditional }\end{array}$ & 26 & - & - & - & - & - & - & - \\
\hline & $\begin{array}{l}\text { C2: parent } \\
\text { - diet and } \\
\text { activity } \\
\text { substitute }\end{array}$ & 26 & - & - & - & - & - & - & - \\
\hline \multirow[t]{2}{*}{$\begin{array}{l}\text { Magarey } \\
2011\end{array}$} & $\begin{array}{l}\text { I: par- } \\
\text { ent healthy } \\
\text { lifestyle }\end{array}$ & 85 & - & - & - & - & - & - & - \\
\hline & $\begin{array}{l}\text { C: healthy } \\
\text { lifestyle }\end{array}$ & 84 & - & - & - & - & - & - & - \\
\hline $\begin{array}{l}\text { Jansen } \\
2011\end{array}$ & $\begin{array}{l}\text { I: parent } \\
\text { CBT }\end{array}$ & 59 & - & - & - & - & - & - & - \\
\hline
\end{tabular}




\begin{tabular}{|c|c|c|c|c|c|c|c|c|c|}
\hline & $\begin{array}{l}\text { C: waiting } \\
\text { list control }\end{array}$ & 39 & - & - & - & - & - & - & - \\
\hline \multirow[t]{3}{*}{$\begin{array}{l}\text { Collins } \\
2011\end{array}$} & $\begin{array}{l}\text { I: parent- } \\
\text { only diet }\end{array}$ & 63 & - & - & - & - & - & - & - \\
\hline & $\begin{array}{l}\mathrm{C} 1 \text { : par- } \\
\text { ent-child } \\
\text { (physical } \\
\text { activity) }\end{array}$ & 73 & - & - & - & - & - & - & - \\
\hline & $\begin{array}{l}\text { C2: par- } \\
\text { ent-child } \\
\text { (phys- } \\
\text { ical activity } \\
+ \text { diet) }\end{array}$ & 70 & - & - & - & - & - & - & - \\
\hline \multirow[t]{2}{*}{$\begin{array}{l}\text { Boutelle } \\
2011\end{array}$} & $\begin{array}{l}\text { I: parent- } \\
\text { only }\end{array}$ & 40 & - & - & - & - & - & - & - \\
\hline & $\begin{array}{l}\text { C: parent- } \\
\text { child }\end{array}$ & 40 & - & - & - & - & - & - & - \\
\hline \multirow[t]{2}{*}{ West 2010} & $\begin{array}{l}\text { I: parent- } \\
\text { only }\end{array}$ & 52 & - & - & - & - & - & - & - \\
\hline & C: control & 49 & - & - & - & - & - & - & - \\
\hline \multirow[t]{2}{*}{$\begin{array}{l}\text { Resnick } \\
2009\end{array}$} & $\begin{array}{l}\text { I: educa- } \\
\text { tional ma- } \\
\text { terial } \\
+ \text { personal } \\
\text { encounters }\end{array}$ & 22 & - & - & - & - & - & - & - \\
\hline & $\begin{array}{l}\text { C: educa- } \\
\text { tional ma- } \\
\text { terial }\end{array}$ & 24 & - & - & - & - & - & - & - \\
\hline \multirow[t]{3}{*}{$\begin{array}{l}\text { Es- } \\
\text { tabrooks } \\
2009\end{array}$} & $\begin{array}{l}\text { I: par- } \\
\text { ent group } \\
+ \text { IVR } \\
\end{array}$ & 85 & - & - & - & - & - & - & - \\
\hline & $\begin{array}{l}\text { I2: parent } \\
\text { group }\end{array}$ & 85 & - & - & - & - & - & - & - \\
\hline & $\begin{array}{l}\text { C: parent } \\
\text { workbook }\end{array}$ & 50 & - & - & - & - & - & - & - \\
\hline
\end{tabular}




\begin{tabular}{|c|c|c|c|c|c|c|c|c|c|}
\hline \multirow[t]{2}{*}{$\begin{array}{l}\text { Munsch } \\
2008\end{array}$} & $\begin{array}{l}\text { I: mother } \\
\text { only }\end{array}$ & 25 & - & - & - & - & - & - & - \\
\hline & $\begin{array}{l}\text { C: mother- } \\
\text { child }\end{array}$ & 31 & - & - & - & - & - & - & - \\
\hline \multirow[t]{3}{*}{$\begin{array}{l}\text { Janicke } \\
2008\end{array}$} & $\begin{array}{l}\text { I: parent- } \\
\text { only }\end{array}$ & 34 & - & - & - & - & - & - & - \\
\hline & $\begin{array}{l}\text { C1: par- } \\
\text { ent-child }\end{array}$ & 33 & - & - & - & - & - & - & - \\
\hline & $\begin{array}{l}\text { C2: } \\
\text { waiting list } \\
\text { control }\end{array}$ & 26 & - & - & - & - & - & - & - \\
\hline \multirow[t]{3}{*}{$\begin{array}{l}\text { Golley } \\
2007\end{array}$} & $\begin{array}{l}\text { I: parent + } \\
\text { lifestyle ed- } \\
\text { ucation }\end{array}$ & 38 & - & - & - & - & - & - & - \\
\hline & $\mathrm{C} 1$ : parent & 37 & - & - & - & - & - & - & - \\
\hline & $\begin{array}{l}\text { C2: } \\
\text { waiting list } \\
\text { control }\end{array}$ & 36 & - & - & - & - & - & - & - \\
\hline \multirow[t]{2}{*}{$\begin{array}{l}\text { Golan } \\
2006\end{array}$} & $\begin{array}{l}\text { I: parent- } \\
\text { only }\end{array}$ & 14 & - & - & - & - & - & - & - \\
\hline & $\begin{array}{l}\text { C: parent- } \\
\text { child }\end{array}$ & 18 & - & - & - & - & - & - & - \\
\hline \multirow[t]{3}{*}{$\begin{array}{l}\text { Aragona } \\
1975\end{array}$} & $\begin{array}{l}\text { I1: parent- } \\
\text { only + re- } \\
\text { inforce- } \\
\text { ment }\end{array}$ & 5 & - & - & - & - & - & - & - \\
\hline & $\begin{array}{l}\text { I2: parent- } \\
\text { only }\end{array}$ & 5 & - & - & - & - & - & - & - \\
\hline & C: control & 5 & - & - & - & - & - & - & - \\
\hline \multicolumn{10}{|c|}{$\begin{array}{l}\text { “_” denotes not reported } \\
\text { C: comparator; CBT: cognitive behavioural therapy; I: intervention; IVR: interactive voice response; NOURISH: Nourishing Our } \\
\text { Understanding of Role Modelling to Improve Support and Health; PCP: primary care providers }\end{array}$} \\
\hline
\end{tabular}




\section{Appendix 9. Checklist to aid consistency and reproducibility of GRADE assessments}

\section{Body mass index (BMI) variables}

\section{Study limitations (risk of bias) $^{a}$}

1. Was random sequence generation used (i.e. Yes no potential for selection bias)?

2. Was allocation concealment used (i.e. no po- Unclear tential for selection bias)?

3. Was there blinding of participants and per- Unclear sonnel (i.e. no potential for performance bias)?

4. Was there blinding of outcome assessment Unclear (i.e. no potential for detection bias)?

5. Was an objective outcome used? Yes

6. Were $>80 \%$ of participants enrolled in trials No () included in the analysis (i.e. no potential reporting bias)?

7. Were data reported consistently for the out- No () come of interest (i.e. no potential selective reporting)?

8. No other biases reported (i.e. no potential of Yes other bias)?

9. Did the trials end up as scheduled (i.e. not Yes stopped early)?

Inconsistency $^{b}$

\begin{abstract}
1. Point estimates did not vary widely?
2. To what extent did confidence intervals overlap (substantial: all confidence intervals overlap at least 1 of the included studies point estimate; some: confidence intervals overlap but not all overlap at least 1 point estimate; no: at least 1 outlier: where the confidence interval of some of the studies do not overlap with those of most included studies)?
\end{abstract}

3. Was the direction of effect consistent? No

4. What was the magnitude of statistical het- Low erogeneity (as measured by $\left.\mathrm{I}^{2}\right)$ - low $\left(\mathrm{I}^{2}<40 \%\right)$ , moderate $\left(\mathrm{I}^{2} 40-60 \%\right)$, high $\left.\mathrm{I}^{2}>60 \%\right)$ ? 
5. Was the test for heterogeneity statistically Not statistically significant significant $(\mathrm{P}<0.1)$ ?

\section{Indirectness}

Imprecision $^{d}$
1. Were the populations in included studies ap- Highly applicable plicable to the decision context?

2. Were the interventions in the included stud- Highly applicable ies applicable to the decision context?

3. Was the included outcome not a surrogate No (however, only relevant outcome) outcome?

4. Was the outcome timeframe sufficient? Sufficient

5. Were the conclusions based on direct com- Yes parisons?

1. Was the confidence interval for the pooled No () estimate consistent with benefit?

2. What is the magnitude of the median sam- Low to intermediate () ple size (high: > 300 participants, intermediate: 100-300 participants, low: $<100$ participants) ?e

3. What was the magnitude of the number of Small () included studies (large: $>10$ studies, moderate: 5-10 studies, small: $<5$ studies)?

4. Was the outcome a common event (e.g. oc- N/A curs more than $1 / 100)$ ?

\section{Publication biased}

2. Was grey literature searched? Yes

3. Were no restrictions applied to study selec- Yes tion on the basis of language?

4. There was no industry influence on studies Yes included in the review?

5. There was no evidence of funnel plot asym- Unclear metry?

6. There was no discrepancy in findings be- Unclear tween published and unpublished trials? 
${ }^{a}$ Questions on risk of bias are answered in relation to the majority of the aggregated evidence in the meta-analysis rather than to individual trials.

${ }^{b}$ Questions on inconsistency are primarily based on visual assessment of forest plots and the statistical quantification of heterogeneity based on $\mathrm{I}^{2}$.

${ }^{c}$ When judging the width of the confidence interval it is recommended to use a clinical decision threshold to assess whether the imprecision is clinically meaningful.

${ }^{d}$ Questions address comprehensiveness of the search strategy, industry influence, funnel plot asymmetry and discrepancies between published and unpublished trials.

${ }^{e}$ Depends on the context of the systematic review area.

(): key item for possible downgrading the quality of the evidence (GRADE) as shown in the footnotes of the 'Summary of finding' table(s); GRADE: Grading of Recommendations Assessment, Development and Evaluation; N/A: not applicable

\section{Appendix 10. Survey of study investigators providing information on included trials}

\begin{tabular}{|c|c|c|c|c|}
\hline & $\begin{array}{l}\text { Study author contacted } \\
\text { (DD/MM/YYYY) }\end{array}$ & $\begin{array}{l}\text { Study author replied } \\
\text { (DD/MM/YYYY) }\end{array}$ & $\begin{array}{l}\text { Study author asked for } \\
\text { additional information } \\
\text { (short summary) }\end{array}$ & $\begin{array}{l}\text { Study author provided } \\
\text { data } \\
\text { (short summary) }\end{array}$ \\
\hline Resnicow 2015 & $15 / 11 / 2015$ & - & - & - \\
\hline Mazzeo 2014 & $10 / 08 / 2015$ & $10 / 08 / 2015$ & $\begin{array}{l}\text { Allocation concealment } \\
\text { approach and blinding of } \\
\text { outcome assessors }\end{array}$ & - \\
\hline van Grieken 2013 & $10 / 08 / 2015$ & $10 / 08 / 2015$ & $\begin{array}{l}\text { Allocation concealment } \\
\text { approach and selective re- } \\
\text { porting of outcomes }\end{array}$ & Yes, 14/09/2015 \\
\hline Small 2013 & $11 / 08 / 2015$ & - & N/A & N/A \\
\hline Esfarjani 2013 & $11 / 08 / 2015$ & - & N/A & N/A \\
\hline Moens 2012 & $18 / 08 / 2015$ & $25 / 08 / 2015$ & $\begin{array}{l}\text { Details blinding of partic- } \\
\text { ipants, personnel and out- } \\
\text { come assessors }\end{array}$ & Yes, 03/09/15 \\
\hline Raynor 2012a & $20 / 11 / 2014$ & $25 / 11 / 2014$ & $\begin{array}{l}\text { Mean and standard devi- } \\
\text { ation of the BMI } \mathrm{z} \text { scores } \\
\text { for each of the } 3 \text { arms in } \\
\text { the trial }\end{array}$ & Yes, 25/11/2014 \\
\hline Raynor 2012b & $20 / 11 / 2014$ & $25 / 11 / 2014$ & $\begin{array}{l}\text { Mean and standard devi- } \\
\text { ation of the BMI } \mathrm{z} \text { scores } \\
\text { for each of the } 3 \text { arms in } \\
\text { the trial }\end{array}$ & Yes, 25/11/2014 \\
\hline
\end{tabular}


(Continued)

\begin{tabular}{|c|c|c|c|c|}
\hline Magarey 2011 & $11 / 08 / 2015$ & $11 / 08 / 2015$ & N/A & N/A \\
\hline Jansen 2011 & $11 / 08 / 2015$ & $11 / 08 / 2015$ & $\begin{array}{l}\text { Randomi- } \\
\text { sation procedures, blind- } \\
\text { ing of outcome assessors, } \\
\text { selective reporting of out- } \\
\text { comes }\end{array}$ & - \\
\hline Collins 2011 & $11 / 08 / 2015$ & $11 / 08 / 2015$ & $\begin{array}{l}\text { Allocation concealment } \\
\text { approach }\end{array}$ & - \\
\hline Boutelle 2011 & $11 / 08 / 2015$ & - & N/A & N/A \\
\hline West 2010 & $11 / 08 / 2015$ & $12 / 08 / 2015$ & $\begin{array}{l}\text { Selective reporting of out- } \\
\text { comes }\end{array}$ & - \\
\hline Resnick 2009 & $11 / 08 / 2015$ & - & N/A & $\mathrm{N} / \mathrm{A}$ \\
\hline Estabrooks 2009 & $11 / 08 / 2015$ & - & N/A & $\mathrm{N} / \mathrm{A}$ \\
\hline Munsch 2008 & $16 / 04 / 2014$ & $16 / 04 / 2014$ & $\begin{array}{l}\text { Additional data for Child } \\
\text { Behaviour Checklist and } \\
\text { English version of study } \\
\text { publication }\end{array}$ & Yes, 16/04/2014 \\
\hline Janicke 2008 & $11 / 08 / 2015$ & $11 / 08 / 2015$ & $\begin{array}{l}\text { Allocation conceal- } \\
\text { ment approach, blinding } \\
\text { of outcome assessors }\end{array}$ & Yes, 24/08/15 \\
\hline Golley 2007 & $11 / 08 / 2015$ & $11 / 08 / 2015$ & N/A & N/A \\
\hline Golan 2006 & $11 / 08 / 2015$ & - & N/A & N/A \\
\hline Aragona 1975 & No email address & - & N/A & N/A \\
\hline
\end{tabular}

\section{Appendix I I. Health-related quality of life: instruments}

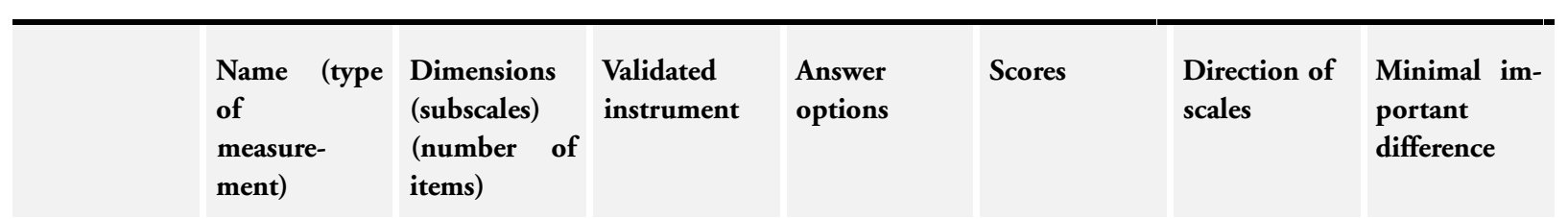




\begin{tabular}{|c|c|c|c|c|c|c|c|}
\hline Mazzeo 2014 & $\begin{array}{l}\text { Pediatric } \\
\text { Health-Re- } \\
\text { lated Quality } \\
\text { of Life (Ped- } \\
\text { sQL4.0) }\end{array}$ & $\begin{array}{l}4 \text { (physical ( } 8 \\
\text { items), emo- } \\
\text { tional } \\
\text { (5 items), so- } \\
\text { cial ( } 5 \text { items), } \\
\text { and school ( } 5 \\
\text { items)) }\end{array}$ & Yes & $\begin{array}{l}\text { 5-point Likert } \\
\text { scale } \\
\text { from } 0 \text { (never) } \\
\text { to } 4 \text { (almost al- } \\
\text { ways) }\end{array}$ & $\begin{array}{l}\text { Scores are } \\
\text { transformed } \\
\text { on a scale from } \\
0 \text { to } 100\end{array}$ & $\begin{array}{l}\text { Higher scores } \\
\text { indicate better } \\
\text { HRQoL }\end{array}$ & Unknown \\
\hline
\end{tabular}

HRQoL: health-related quality of life

\section{H I S T O R Y}

Review first published: Issue 12, 2015

\begin{tabular}{l|l|l}
\hline Date & Event & Description \\
\hline 11 October 2008 & New citation required and conclusions have changed & $\begin{array}{l}\text { This review concludes that combined behavioural } \\
\text { lifestyle interventions compared to standard care or self- } \\
\text { help can produce a significant and clinically meaningful } \\
\text { reduction in overweight in children and adolescents } \\
\text { The search was updated to May 2008. Some amend- } \\
\text { ments were made to update the search strategies. No } \\
\text { changes have been made to other aspects of the method- } \\
\text { ology. Forty-six new studies have been included. These } \\
\text { included information on drug interventions for treating } \\
\text { obesity in adolescents. The added evidence suggests that } \\
\text { lifestyle interventions appear to have positive effects in } \\
\text { the treatment of child and adolescent obesity. Further- } \\
\text { more, orlistat and sibutramine were found to have bene- } \\
\text { ficial effects on adiposity in obese adolescents. However, } \\
\text { a range of adverse effects was noted }\end{array}$ \\
\hline
\end{tabular}




\section{CONTRIBUTIONSOFAUTHORS}

Emma Loveman (EL): acquiring trial reports, trial selection, data extraction, data analysis, data interpretation, review draft and future review updates.

Lena Al-Khudairy (LAK): acquiring trial reports, trial selection and data extraction.

Rebecca E Johnson (RJ): acquiring trial reports, trial selection and data extraction.

Wendy Robertson (WR): acquiring trial reports, trial selection, review draft and future review updates.

Jill L Colquitt (JC): acquiring trial reports, trial selection and data extraction.

Emma L Mead (EM): acquiring trial reports and trial selection.

Louisa J Ells (LE): acquiring trial reports and trial selection.

Maria-Inti Metzendorf (MIM): search strategy development and review draft.

Karen Rees (KR): project management, trial selection, resolving discrepancies, data interpretation, review draft and future review updates.

\section{DECLARATIONSOF INTEREST}

EL: none known.

LAK: none known.

RJ: none known.

WR: none known.

JC: none known.

EM: none known.

LE: none known.

MIM: none known.

KR: none known.

\section{SOURCES OF SUPPORT}

\section{Internal sources}

- Division of Health Sciences, Warwick Medical School, University of Warwick, UK.

- Health and Social Care Institute, Teesside University, UK.

- Effective Evidence LLP, UK. 


\section{External sources}

- NIHR Cochrane Programme Grant, UK.

- Karen Rees, Lena Al-Khudairy, Rebecca Johnson and Wendy Robertson are also supported by the National Institute for Health Research Collaboration for Leadership in Applied Health Research and Care West Midlands at University Hospitals Birmingham NHS Foundation Trust, UK.

\section{DIFFERENCES BETWEEN PROTOCOLANDREVIEW}

Given the rapid growth in the treatment of child and adolescent obesity, the original review was split into six separate reviews, with a specific intervention and age focus.

- Diet, physical activity and behavioural interventions for the treatment of overweight or obesity in adolescents aged 12 to 17 years.

- Diet, physical activity and behavioural interventions for the treatment of overweight or obesity in children aged 5 to 11 years.

- Diet, physical activity and behavioural interventions for the treatment of overweight or obesity in infants aged 0 to 4 years.

- Drug interventions for the treatment of obesity in children and adolescents.

- Parent-only interventions for childhood overweight or obesity.

- Surgery for the treatment of obesity in children and adolescents.

For lifestyle interventions, we included only randomised controlled trials that were specifically designed to treat obesity in children and observed participants for a minimum of six months. The rationale for introducing this criterion arose from the belief that many interventions appear to be effective in the short term (up to three months), but not in the long term (Glenny 1997). It seemed to be more important to evaluate the longer-term effects of treatments, as this would provide a more valuable indication of effectiveness, given the chronic nature of obesity.

\section{NOTES}

Part of the 'Background', Methods' section, appendices, additional tables and figures 1 to 3 of this review are based on a standard template established by the Cochrane Metabolic and Endocrine Disorders Group. 